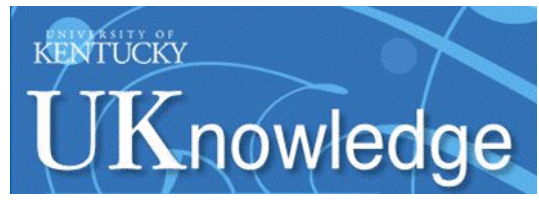

University of Kentucky

UKnowledge

2010

\title{
QUALITY ANALYSIS IN FLEXIBLE MANUFACTURING SYSTEMS WITH BATCH PRODUCTIONS
}

Junwen Wang

University of Kentucky, junwen.wang@uky.edu

Right click to open a feedback form in a new tab to let us know how this document benefits you.

\section{Recommended Citation}

Wang, Junwen, "QUALITY ANALYSIS IN FLEXIBLE MANUFACTURING SYSTEMS WITH BATCH PRODUCTIONS" (2010). University of Kentucky Doctoral Dissertations. 51.

https://uknowledge.uky.edu/gradschool_diss/51

This Dissertation is brought to you for free and open access by the Graduate School at UKnowledge. It has been accepted for inclusion in University of Kentucky Doctoral Dissertations by an authorized administrator of UKnowledge. For more information, please contact UKnowledge@lsv.uky.edu. 


\title{
ABSTRACT OF DISSERTATION
}

\author{
Junwen Wang
}

The Graduate School

University of Kentucky 


\title{
QUALITY ANALYSIS IN FLEXIBLE MANUFACTURING SYSTEMS WITH BATCH PRODUCTIONS
}

\author{
ABSTRACT OF DISSERTATION \\ A dissertation submitted in partial fulfillment of the \\ requirements for the degree of Doctor of Philosophy in the \\ College of Engineering \\ at the University of Kentucky
}

\author{
By \\ Junwen Wang \\ Lexington, Kentucky
}

Director: Dr. Jingshan Li, Assistant Professor of Department of Electrical and Computer Engineering

Lexington, Kentucky

2010

Copyright(C)Junwen Wang 2010 


\section{ABSTRACT OF DISSERTATION}

\section{QUALITY ANALYSIS IN FLEXIBLE MANUFACTURING SYSTEMS WITH BATCH PRODUCTIONS}

To improve product quality and reduce cost, batch production is often implemented in many flexible manufacturing systems. However, the current literature does not provide any method to analyze the quality performance in a flexible manufacturing system with batch production.

In this research, we present an analytical method with closed-form formula to evaluate the quality performance in such systems. Based on the model, we discover and investigate monotonic and non-monotonic properties in quality to provide practical guidance for operation management. To improve product quality, we introduce the notions of quality improvability with respect to product sequencing. In addition, we develop the indicators for quality improvability based on the data available on the factory floor rather than complicated calculations. We define the bottleneck sequence and bottleneck transition as the ones that impede quality in the strongest manner, investigate the sensitivity of quality performance with respect to sequences and transitions, and propose quality bottleneck sequence and transition indicators based on the measured data. Finally, we provide a case study at an automotive paint shop to show how this method is applied to improve paint quality.

Moreover, we explore a potential application to reduce energy consumption and atmospheric emissions at automotive paint shops. By selecting appropriate batch and sequence policies, the paint quality can be improved and repaints can be reduced so that less material and energy will be consumed, and less atmospheric emissions will be generated. It is shown that such scheduling and control method can lead to significant energy savings and emission reduction with no extra investment nor changes to existing painting processes.

The successful development of such method would open up a new area in manufacturing systems research and contribute to establish a solid foundation for an integrated study on productivity, quality and flexibility. In addition, it will provide production engineers and operation managers a quantitative tool for continuous improvement on product quality in flexible manufacturing environment. 
KEYWORDS: Flexible Manufacturing System, Batch Production, Markov Chain, Quality Analysis, Automotive Paint Shop

Student's Signature

June 21, 2010

Date 


\title{
QUALITY ANALYSIS IN FLEXIBLE MANUFACTURING SYSTEMS WITH BATCH PRODUCTIONS
}

\author{
By \\ Junwen Wang
}

Dr. Jingshan Li

Director of Dissertation

Dr. Stephen Gedney

Director of Graduate Studies

June 21, 2010

Date 


\section{RULES FOR THE USE OF DISSERTATIONS}

Unpublished dissertations submitted for the Doctor's degree and deposited in the University of Kentucky Library are as a rule open for inspection, but are to be used only with due regard to the rights of the authors. Bibliographical references may be noted, but quotations or summaries of parts may be published only with the permission of the author, and with the usual scholarly acknowledgments.

Extensive copying or publication of the dissertation in whole or in part also requires the consent of the Dean of the Graduate School of the University of Kentucky.

A library which borrows this dissertation for use by its patrons is expected to secure the signature of each user.

Name

Date 


\title{
DISSERTATION
}

\author{
Junwen Wang
}

The Graduate School

University of Kentucky 


\section{QUALITY ANALYSIS IN FLEXIBLE MANUFACTURING SYSTEMS WITH BATCH PRODUCTIONS}

\begin{tabular}{l}
\hline DISSERTATION \\
\hline
\end{tabular}

A dissertation submitted in partial fulfillment of the requirements for the degree of Doctor of Philosophy in the

College of Engineering at the University of Kentucky

By

Junwen Wang

Lexington, Kentucky

Director: Dr.Jingshan Li, Assistant Professor of Department of Electrical and Computer Engineering

Lexington, Kentucky

Copyright@Junwen Wang 2010 
I dedicate this dissertation to my family. 


\section{ACKNOWLEDGMENTS}

I would like to express my sincere gratitude to my advisor, Dr. Jingshan Li, for his technical direction and support throughout the $\mathrm{PhD}$ study. I have enjoyed this research under his experienced and visionary supervision. I would also like to thank Dr. Jorge Arinez, Dr. Stephan Biller, and Dr. Ningjian Huang from General Motors R\&D Center for their cooperation. Also, I thank the financial support of NSF GOALI.

I would also like to thank my committee members, Dr. YuMing Zhang, Dr. Lawrence Holloway, and Dr. Dusan Sekulic, for their support and valuable comments to my dissertation.

I thank my parents. Without their love and support, my graduate studies would not be possible. I also thank my fiancée, Xingxing, for her understanding, support and encouragement.

I appreciate the sincerity and support from these people mentioned here and others as well. 


\section{TABLE OF CONTENTS}

ACKNOWLEDGMENTS ........................ iii

LIST OF TABLES . . . . . . . . . . . . . . . . . . . . . . . . . . v vi

LIST OF FIGURES . . . . . . . . . . . . . . . . . . . . . . vii

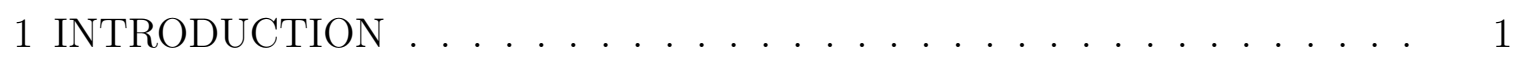

1.1 Motivation . . . . . . . . . . . . . . . . . . . . . 1

1.2 Methodology . . . . . . . . . . . . . . . . . . 7

2 LITERATURE REVIEW . . . . . . . . . . . . . . . . . . . . . . . . . . . . 10

2.1 Flexibility and Quality . . . . . . . . . . . . . . . . . . . 10

2.2 Batch Production and Quality . . . . . . . . . . . . . . . . . . . . . . 13

2.3 EEEF and Quality . . . . . . . . . . . . . . . . . . . . . . . . . . . . . . . . . . . . .

2.4 Summary . . . . . . . . . . . . . . . . . . . . . . . . . . . . . . . 19

3 ANALYSIS OF QUALITY IN FLEXIBLE SYSTEMS . . . . . . . . . . . . 21

3.1 Modeling based on Markov Chain . . . . . . . . . . . . . . . . . 21

3.2 Two Product Types with Batch Size Two . . . . . . . . . . . . . . . . 24

3.2.1 Analytical expressions . . . . . . . . . . . . . . . . . 24

3.2 .2 Monotonicity . . . . . . . . . . . . . . . . . . . . 27

3.2.3 Comparisons with other sequencing policies . . . . . . . . . . 27

3.3 General Multiple Products Multiple Batch Sizes Case . . . . . . . . . 29

3.3 .1 General formulas . . . . . . . . . . . . . . . . . . . 29

3.3 .2 Bernoulli quality reliability case . . . . . . . . . . . . . . 32

3.3.3 Monotonic and non-monotonic properties with respect to quality failure and repair probabilities . . . . . . . . . . . 33

3.3 .4 Asymptotic properties . . . . . . . . . . . . . . . . 35

3.3 .5 Oscillating properties with respect to batch size . . . . . . . . 39

3.3.6 Non-monotonic properties in non-equal products case . . . . . 44

3.4 Summary . . . . . . . . . . . . . . . . . . . 46

4 PRODUCT SEQUENCING WITH RESPECT TO QUALITY . . . . . . . 48

4.1 Two Product Types . . . . . . . . . . . . . . . . . 48

$4.1 .1 \quad$ Quality performance in different sequencing policies . . . . . . 48

4.1.2 $\quad$ Sequencing comparison under Bernoulli-like assumption. . . . 51

4.1.3 Sequencing comparison under Bernoulli-relax assumption . . . 52

$4.2 \quad$ General Multiple Product Types . . . . . . . . . . . . . . . . 53

4.2.1 Quality performance in different sequencing policies . . . . . . 53

4.2 .2 A paint shop example ...................... 57

$4.2 .3 \quad$ Product sequencing under Bernoulli-like assumption . . . . . . 60

4.2 .4 Product sequencing under Bernoulli-relax assumption . . . . . 64

4.3 Quality Improvability with respect to Product Sequencing . . . . . . 71

$4.3 .1 \quad$ Quality improvability under Bernoulli-like assumption. . . . . 72 
4.3.2 Quality improvability under Bernoulli-relax assumption . . . . 74

4.4 Summary . . . . . . . . . . . . . . . . . . . . . 87

5 BOTTLENECK TRANSITION WITH RESPECT TO QUALITY . . . . . 89

5.1 Implications from Simple Cases . . . . . . . . . . . . . . . . 90

$5.1 .1 \quad$ Three product types in strictly sequencing policy . . . . . . . 90

5.1.2 $\quad$ Two product types three batch sizes in batch policy . . . . . . 91

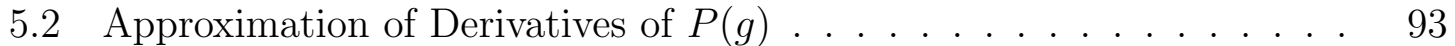

5.3 Transition Bottleneck Indicators . . . . . . . . . . . . . . . . . . . . . 97

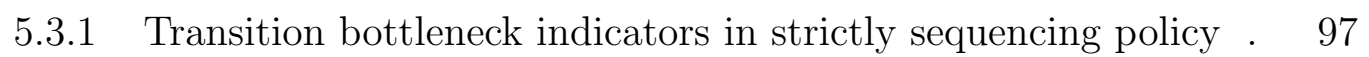

5.3 .2 Transition bottleneck indicators in batch policy . . . . . . . . 98

$5.3 .3 \quad$ Numerical justification . . . . . . . . . . . . . . . . . 100

5.4 Summary $\ldots \ldots \ldots \ldots$. . . . . . . . . . . . . . . . . 103

6 PRODUCT SEQUENCING TO REDUCE ENERGY CONSUMPTION AND

ATMOSPHERIC EMISSIONS . . . . . . . . . . . . . . . . . . . . . 104

6.1 Job Flow, Energy and Emission Evaluation in Paint Shops . . . . . . 104

6.1 .1 Job flow . . . . . . . . . . . . . . . . 104

$6.1 .2 \quad$ Energy evaluation . . . . . . . . . . . . . . . . 106

6.1 .3 Emission evaluation . . . . . . . . . . . . . . . . . . . . . . 108

6.2 Evaluation of Energy Usage and Atmospheric Emission in Paint Shops 108

6.2 .1 Comparison of energy usage . . . . . . . . . . . . . . 108

6.2 .2 Emission Comparison . . . . . . . . . . . . . . . . . . . . . 113

6.3 Summary . . . . . . . . . . . . . . . . . . . 117

7 CASE STUDY . . . . . . . . . . . . . . . . . . . . . . . . 119

8 CONCLUSIONS . . . . . . . . . . . . . . . . . . . . . . . . . . . 121

APPENDICES . . . . . . . . . . . . . . . . . . . . 126

REFERENCES . . . . . . . . . . . . . . . . . . . . . . . . . . 181

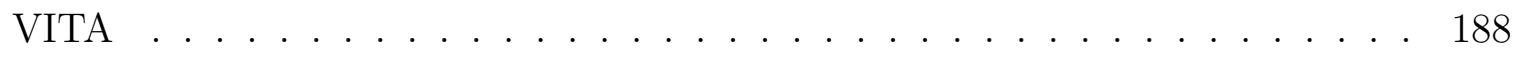




\section{LIST OF TABLES}

3.1 Non-monotonicity of $P(g)$ with respect to $\lambda_{11} \ldots \ldots$. . . . . . . . . . . . 34

3.2 Non-monotonicity of $P(g)$ with respect to $\mu_{11}$. . . . . . . . . . . . . . . . 34

3.3 Necessary condition for oscillation . . . . . . . . . . . . . . . . . . 42

3.4 Percentage of non-monotonic cases with respect to quality failure and repair

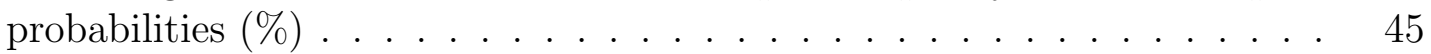

3.5 Percentage of non-monotonic cases with respect to increasing batch size (\%) 45

4.1 Correctness of condition (4.57) (\%) . . . . . . . . . . . . 66

4.2 Errors when condition (4.57) has incorrect selections . . . . . . . . . . . . 67

\begin{tabular}{|ll|l|l|l|l|}
\hline 4.3 & Correctness of Proposition & 4.5 & under Bernoulli-relax assumption \\
\hline
\end{tabular}

\begin{tabular}{|ll|l|l|}
\hline 4.4 & Correctness of Proposition & 4.6 & under Bernoulli-relax assumption with iden- \\
\hline
\end{tabular}

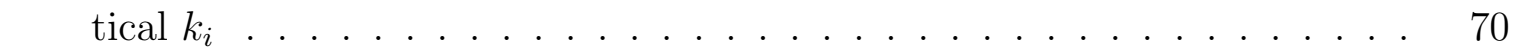

\begin{tabular}{|ll|l|l|}
\hline 4.5 & Correctness of Proposition & 4.7 & under Bernoulli-relax assumption with ran- \\
\hline
\end{tabular}

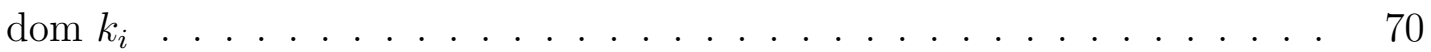

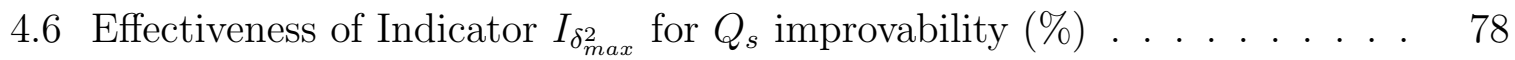

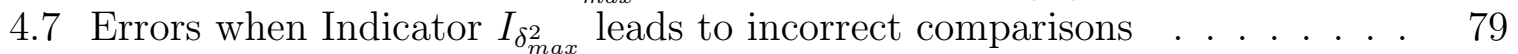

4.8 Effectiveness of Indicator $I_{\delta_{\max }^{2}}$ for $Q_{s}$ unimprovable sequence (\%) $\quad$. . . . 80

4.9 Errors when Indicator $I_{\delta_{\max }^{2}}$ identifies incorrect unimprovable sequence . . 80

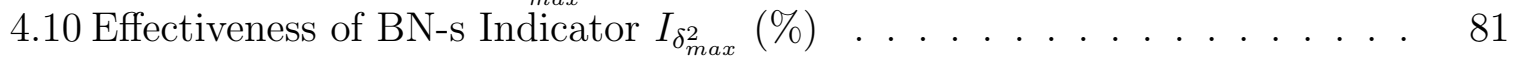

4.11 Errors when Indicator $I_{\delta_{\max }^{2}}$ results in incorrect BN-s identification . . . . 81

4.12 Effectiveness of Indicator $I_{\delta_{\max }}$ for $Q_{s}$ improvability (\%)

4.13 Errors when Indicator $I_{\delta_{\max }}$ leads to incorrect comparisons . . . . . . . . 84

4.14 Effectiveness of Indicator $I_{\delta_{\max }}$ for $Q_{s}$ unimprovable sequence (\%) $\quad$. . . . 85

4.15 Errors when Indicator $I_{\delta_{\max }}$ identifies incorrect unimprovable sequence . . 85

4.16 Effectiveness of BN-s Indicator $I_{\delta_{\max }}(\%) \ldots \ldots \ldots$. . . . . . . . 86

4.17 Errors when Indicator $I_{\delta_{\max }}$ results in incorrect BN-s identification . . . . 87

5.1 Effectiveness of n-BN-t and p-BN-t indicators in strictly sequencing policy (\%) 101

5.2 Errors when n-BN-t and p-BN-t indicators lead to incorrect comparisons

in strictly sequencing policy . . . . . . . . . . . . . . 101

5.3 Effectiveness of n-BN-t and p-BN-t indicators in batch policy(\%) . . . . . 102

5.4 Errors when n-BN-t and p-BN-t indicators lead to incorrect comparisons

in batch policy . . . . . . . . . . . . . . . . . 102 


\section{LIST OF FIGURES}

3.1 State transition diagram of two-product type case with batch size two . . 25

3.2 Product quality as a function of batch size in Bernoulli case . . . . . . . . 33

3.3 Asymptotic behavior: Case 1 . . . . . . . . . . . . . . . . . . . . . 35

3.4 Asymptotic behavior: Case 2 . . . . . . . . . . . . . . . . . . . . . . . . 36

3.5 Asymptotic behavior: Cases 3.1 and 3.2 . . . . . . . . . . . . . . . . . . . 38

$3.6 \quad D_{k+1}-D_{k}$ vs $a$ and $b$ with respect to different odd batch size $k$. . . . . . 41

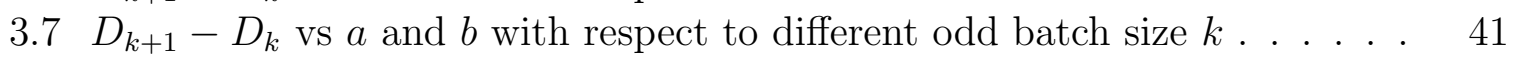

3.8 Oscillation boundaries with various batch sizes . . . . . . . . . . . . . . . 42

$3.9 \quad D_{k+2}-D_{k}$ vs $a$ and $b$ with respect to different batch sizes . . . . . . . . . 44

6.1 Illustration of job flow in paint in automotive assembly plant . . . . . . . 104

6.2 Simplified painting process . . . . . . . . . . . . . . 106

6.3 $\quad$ Energy usage of optimal sequence in batch policy vs worst sequence in strictly sequencing policy . . . . . . . . . . . . . . . . 110

6.4 Energy usage of optimal sequence in batch policy vs optimal sequence in strictly sequencing policy . . . . . . . . . . . . . . . . 111

6.5 Energy usage of optimal sequence vs worst sequence in batch policy . . . 112

6.6 Energy usage of optimal sequence in batch policy vs randomly mixed policy 113

6.7 Emissions: optimal sequence in batch policy vs worst sequence in strictly sequencing policy . . . . . . . . . . . . . . . . 115

6.8 Emissions: optimal sequence in batch policy vs optimal sequence in strictly sequencing policy ......................... 116

6.9 Emission: optimal sequence vs worst sequence in batch policy . . . . . . . 117

6.10 Emissions: optimal sequence in batch policy vs randomly mixed policy. . 118 


\section{CHAPTER 1 INTRODUCTION}

Considering the growing importance of flexibility in manufacturing, the number of flexible manufacturing systems is increasing. Many of them use batch production to improve productivity and product quality. This chapter illustrates the motivation of this research by overviewing the current work on quality, flexibility and batch production, and outlines the analytical framework to investigate product quality in flexible manufacturing systems with batch productions.

\subsection{Motivation}

Many manufacturing enterprises have confronted increasing competition on global market over recent years. In order to be more competitive, many companies have increased manufacturing flexibility to improve production efficiency, reduce cost, and respond quickly to changing customer preferences. For example, multiple models of vehicles with more options can be built on the same production line in many automotive assembly plants.

Flexible manufacturing systems have been studied intensively during the last twenty years (see, monographs [1]-[4], reviews [5]-[13]), and continue to attract research efforts in modern manufacturing. The issues of investment cost, flexibility measurement, inventory, scheduling, and the tradeoffs between productivity and flexibility, etc., are addressed in most of the flexibility studies. However, the question 
of product quality is usually neglected. It is typically assumed that quality related issues have minimal impact $([14])$. On the other hand, extensive research has been carried out in quality management as well, but independently. The majority of the publications on quality research seek to maintain and improve product quality while ignoring the productivity or flexibility concerns ([15]). Statistical process control, total quality management, quality function deployment, etc., are the main tools in such studies (e.g., see recent reviews [16]-[20]). Little research attention has been paid to investigate the coupling between flexible system design and product quality.

Empirical evidences and analytical studies have shown that production system design and product quality are tightly coupled ([15], [21]-[22]); in particular, flexibility has a significant impact on product quality. Tradeoffs not only exist between flexibility and productivity, but also between flexibility and quality. For example, at an automotive paint shop which is capable of painting different colors, the product (paint) quality is strongly correlated to the number of available paint colors ([23]). Paint quality may temporarily decline after the color change due to incomplete cleaning during purging of the previous colors ([24]). The next vehicles's quality is affected by the previous vehicle's color, in addition to other factors (such as dirty air, vehicle cleaning, equipment maintenance, paint mixing, etc.).

Similar examples can be found in flexible machining lines, welding and assembly systems as well. In flexible machining systems, a flexible fixture restricts and is the core enabler of flexibility of the whole system $([14])$. With the flexible fixture, system flexibility can be achieved to support multiple distinct parts being manufactured (assembled or machined) on the same line with little or no loss of production. In 
the automotive industry, a flexible fixture might be clamps/locators held by robots or other "smart" mobile apparatus. Whenever there is a product change, the fixture needs to adapt itself to the desired corresponding location. The accuracy of the locator variance from its "ideal" location, in many cases, dominates the quality of the products. Reducing the number of adjustment of its location could help to improve product quality ([21]). These examples suggest that flexibility and quality are tightly coupled and that fewer product changes may lead to a better quality.

Therefore, in order to improve product quality as well as shorten changeover time and reduce cost, many flexible manufacturing systems implement batch productions to reduce product changes. In such systems, different types of products are grouped into batches where all parts in each batch have the same type. The change of product type only occurs after the last job in a batch is processed. For example, in automotive paint shops, vehicles with the same color are often grouped into batches to reduce color changes to improve paint quality. In stamping plants, batch processing is implemented with repeated patterns to reduce changeover cost and associated quality losses. In addition, in many welding operations, the welding quality is strongly coupled with part positioning. Similar examples can be found in flexible machining lines where the locating precision of the flexible fixture is a dominant factor in quality. Again batch production is observed in such systems to reduce the quality defects introduced by location errors. Moreover, in some engine assembly lines, different types of engines are typically assembled in batch and changeovers occur on hourly basis. These examples suggest that frequent product changes may impact quality and introducing batch production to reduce losses due to quality degradation during 
changeovers is of importance. Thus, there is a critical need to fully understand the coupling between flexibility and quality in batch productions.

However, the issues of quality in a batch production environment have not been studied. The current literature does not provide a quantitative method to investigate how flexibility in terms of the sequence/batch policies impacts product quality. In spite of substantial research devoted to product sequencing, most of them focus on optimizing setup cost, minimizing makespan and color changes, etc., while the problem of quality is not explicitly discussed. For example, car sequencing and related problems are discovered in some publications ([25]-[30]) to minimize color changes, however, without discussing quality (although it may be implied that minimizing color changes could lead to better paint quality).

To our best knowledge, no research has been discovered to directly study the quality and sequencing issues in flexible manufacturing systems with batch productions in the current literature. As discussed above, different product sequencing and batch policies may result in different quality. In the example of automotive paint shops, it is observed that the darker color has more impact on paint quality than the lighter one because a small amount of darker pigment can adversely affect the lighter color during the cleanup and reconfiguration of the painting guns. To accommodate this, darker vehicles are often sequenced after lighter ones in batch productions. Then, how to determine an appropriate sequence or batch policy? What will be the optimal sequence with respect to quality? Will the quality characteristics change under different policies? Answering such questions can provide insight and guidance for system design and operation to achieve better quality. Therefore, an in-depth study 
to investigate the coupling between flexibility and quality in terms of sequence and batch production policies is needed.

In addition, how to improve quality in flexible manufacturing systems with batch productions has not been addressed. In practice, improving performance is usually accomplished by identifying the bottleneck and improving its operation. Bottleneck identification and mitigation are essential enablers for continuous improvement in manufacturing operations. Most of the studies related to bottlenecks focus on throughput bottlenecks. A system-theoretic method to identify bottleneck by measuring and comparing blockages and starvations has been developed and successfully applied on the factory floor (see monograph [31] and papers [32]-[38]). For the issue of quality, it has been shown in some publications ([21]) that product quality is correlated to both the sequencing policies and the transition probabilities that characterize the changes among good and defective states (where good quality or defective parts are produced during a cycle, respectively). Hence, high product quality can be achieved by avoiding the sequences leading to the worst quality and improving the transitions. Specifically, such improvement can be carried out through identification and mitigation of sequence bottlenecks (BN-s) and transition bottlenecks (BN-t), which are defined as the sequences and transitions that impede product quality in the strongest manner, respectively. In other words, improvement on these sequences or transitions will lead to the largest improvement in product quality compared with improving other sequences or transitions.

Furthermore, improving product quality through optimal batch and sequence scheduling is also beneficial to reduce energy consumption and atmospheric emis- 
sions in manufacturing process. This will provide an alternative approach to achieve energy efficient and environmental friendly (EEEF) manufacturing. It is reported that the US transportation equipment manufacturing industry as a whole (including manufacturing of automobiles and parts, aeroplanes, boats, ships, locomotives, motorcycles, etc.) spent $\$ 3.6$ billion on energy in 1999 ([39]). A huge amount of energy is spent on vehicle assembly. For 37 vehicle assembly plants across the US, it is estimated that energy expenditure in automobile assembly plants is at the level of $\$ 700$ million per year ([39]). Among which, approximately $60 \%$ of total energy in an assembly plant is used by automotive painting, in particular, in painting booths and ovens $([40])$. Thus, automotive painting is not only an important element in vehicle production, but also the largest energy consumption unit throughout the automotive assembly plant. About two-thirds of the energy cost is on electricity, and one-third is on fuel used to generate hot water and steam (for painting booths), as well as heat in ovens. Thus, energy reduction in paint shops has significant importance, even a small percentage. In addition, the painting process results in atmospheric emissions, where some gases such as Carbon Dioxide $\left(\mathrm{CO}_{2}\right)$, Methane $\left(\mathrm{CH}_{4}\right)$, and Nitrous Oxide $\left(\mathrm{N}_{2} \mathrm{O}\right)$ are released into atmosphere, the cleaning of sticky residues on the walls, windows, robots, fixtures, etc., due to overs pray and purge, will result in evaporation of volatile organic compound (VOC) emissions. Such emissions will contribute to the green house effect and adverse impact to environment. Typically, the painting process in automotive paint shops has two functions: protecting the vehicle's body from corrosion and improving the appearance. Vehicle's paint quality is essential for customers, which may directly influence their buying decisions. To achieve good 
paint quality, repeated painting processes may be needed to ensure all defects are corrected. In addition, in order to satisfy the customer preferences and changing demands, a paint shop is typically capable of painting multiple colors, which implies that color changes are often observed in painting process. During the color change, old solvents will be purged out from the painting guns and new paints will be loaded and sprayed. All these lead to additional consumptions of a large amount of energy and painting materials. Beyond these, environmental concerns exist for sprayed and purged paints as well as $\mathrm{CO}_{2}$ equivalent emissions. Therefore, improvement of paint quality to reduce repaints and color changes using optimal sequence scheduling could result in significant reduction in energy consumption and benefits for environment protection.

In summary, the goal of this research is to develop an analytical method to evaluate product quality in a flexible manufacturing system with batch productions, investigate the impacts of sequencing and batch policies with respect to quality, identify the sequence bottlenecks and transition bottlenecks for continuous improvement, and apply the obtained results in an automotive paint shop.

\subsection{Methodology}

In a flexible manufacturing system, the quality performance can be characterized by the probability of producing a good quality part each cycle in steady states. Each product coming out of a flexible machine can be in good states or defective states. The transitions between good states and defective states are determined by the operating

conditions both in current cycle and in previous cycle. In this study, we depict these 
transitions using a discrete Markov chain and we focus on the impacts of the types of products, sequence and batch policies, etc., on product quality.

The main contribution of this research is in the development of a Markov chain model to evaluate the quality performance of a flexible manufacturing system with batch productions (although paper [21] introduces a Markovian approach for analyzing quality, batch production is not addressed). Closed formulas to describe the quality measurement are derived and structural properties (monotonic and non-monotonic properties, asymptotic property, improvabilities, bottlenecks, etc.) to address different batch and sequencing policies are investigated.

The remainder of this dissertation is structured as follows: Chapter 2 reviews related literature on flexibility, quality and batch production. Chapter 3 presents an analytical model to evaluate the quality performance of flexible manufacturing systems with batch operations. By using a Markov chain model, a closed formula to quantify the probability of producing a good part is derived. In addition, the monotonic and non-monotonic properties of product quality with respect to quality failure and repair probabilities and batch sizes are discovered. Chapter 4 investigates the impact of product sequencing on quality. The optimal and bottleneck sequences leading to best and worst quality, respectively, under both batch and strict sequencing policies are obtained, the comparisons between different sequences under different policies are carried out, and finally improvability of product sequencing is studied. Considering that quality performance depends on not only product sequence but also the transition probabilities, Chapter 5 defines the transition bottleneck as the one having the largest impact on quality. Bottleneck indicators based on the collected 
data on the factory floor are developed to identify the bottleneck transitions. Chapter 6 develops an optimal batch and scheduling procedure of vehicles to achieve the goal of energy and emission reduction. Chapter 7 applies this method at an automotive paint shop to evaluate paint quality and investigate potential improvement strategies. Chapter 8 formulates the conclusions. All proofs can be found in the Appendices. 


\section{CHAPTER 2 LITERATURE REVIEW}

In this chapter, Section 2.1 reviews available literature on flexibility related to quality. Section 2.2 introduces the articles on the relationship between quality and batch production in terms of batch size and product sequencing. Section 2.3 introduces studies on energy and emission reduction, and Section 2.4 is the summary.

\subsection{Flexibility and Quality}

During the last twenty years, flexibility has attracted significant amount of research attention. Most of the studies address the tradeoffs between flexibility and productivity by assuming minimal impact on quality. Recently, it has been shown that product quality and product system design are tightly coupled ([15]-[22]).

Paper [14] reviews literature on the measurements of flexibility and their impact on the performance of flexible manufacturing systems. The authors conclude that these studies usually assume that only parts of acceptable quality are produced in flexible manufacturing systems and quality related issues have minimal impact. Instead of investigating inherent relationship between flexibility and quality, the issue of quality is generally discussed at the system and operation level.

Aimed at the widely assertion that flexible manufacturing system positively impacts productivity and quality, paper [41] investigates the issues of flexibility, productivity, and quality from an extensive search and analysis of empirical studies. To 
study possible impact of flexibility on quality, the authors introduce from paper [42] the concept "cost of quality" which is generally easy to quantify and includes the measures employed on the factory floor. Based on total ten projects in eighty four reported quality improvement as claimed, the authors find that there is a relationship between implemented flexibility and improved quality. It implies that tradeoffs exist between flexibility and quality.

Paper [43] addresses the existing literature related to mass customization which is able to provide customized products or services through flexible processes in high volumes. In order to measure the performance of processes or products, many strategies have been implemented such as statistical control charts ([44]) and poka-yoke devices ([45]). Since this flexible system is characterized by single product lots, those quality control strategies based on periodical checking would not be easily adapted. Another problem rising in flexible manufacturing is that a new set of quality characteristics should be defined whenever a product is customized. Therefore, the authors claim that quality control issues should be taken into account when considering product customization and an in-depth study on how to assure quality in mass-customized products is a future research direction.

Due to difficulty in measuring flexibility and quality in production systems, paper [46] defines, quantifies and integrates three measurements of productivity, quality and flexibility using economic impacts. In addition, paper 47] defines flexibility as the function of fuzzy elements such as quality level, efficiency, versatility and availability which implies that flexibility could be implemented at the highest quality, shortest time, lowest cost, and right quantity of output. In particular, quality level is 
described linguistically as low, medium or high. Then the manufacturing flexibility is aggregated by fuzzy elements with different important weight. However, this method can not uncover the intrinsic relationship between flexibility and quality.

Paper [15] explores the intersection of two independent research areas: quality and production system design. In contrast to the conventional wisdom that a product's quality is determined by its design much more than its production, the authors argue that the designed system's flexibility has a significant impact on quality by providing evidence from automotive industry. The authors also suggest several research topics of interest to industry including batch processing and motivate the unexplored research on how flexibility impacts quality.

In manufacturing and other service industries, quality control is usually implemented by incorporating management into inspection technologies. Total Quality Management (TQM) is widely used to ensure products or services to meet customer requirements (see reviews [48]-[51]). Its philosophy on improving quality is that high product quality demands efforts through all levels of an enterprise, from top management to factory work forces. High product quality can be achieved by continuous improvement of processes in manufacturing and services which is chased by the whole organization. In particular, Quality Function Deployment (QFD) and Statistical Process Control (SPC) are two popular techniques to achieve high product quality. QFD is a methodology to product development and design by taking the customer requirements into account (see reviews [18], [52]). An improved version of QFD is proposed to go in hand well with TQM in paper [53]. SPC is used to reduce process variability to achieve high quality (see [54]-[57]) by assuming that quality is inversely proportional 
to process variability. In flexible manufacturing systems, how system flexibility (or variability) impacts quality is still not well understood from the above methods.

Paper [21] studies the impacts of product changes on quality in flexible manufacturing systems using a Markov chain model with randomly mixed products flow. The quantitative model enables us to investigate the correlation between quality and number of products and to predict the quality performance of a flexible manufacturing system. Two sequence policies are compared in the paper and the result suggests that reducing the number of product switch could lead to a better quality performance, which implies that batch production is a possible approach to improve quality.

\subsection{Batch Production and Quality}

Although batch production has been widely used in manufacturing industry, batching in flexible manufacturing systems is typically studied in terms of batch size in scheduling problems.

Paper [58] studies the planning problem of scheduling batches of parts having sequence-dependent setup times in a multi-cell flexible flow shop. Mixed linear programming method is used to minimize makespan given by the completion time of the last batch on the last machine. Paper [59] uses heuristic algorithms to consider batch scheduling problem by minimizing setup costs and the mean flow time. There is an adjusted lookahead-parameter that enables human planners to effectively manage the tradeoff between the two objectives. However, quality is not analyzed in either of them. 
More publications studying product sequencing can be discovered in the literature, however, quality is not explicitly addressed in these papers.

The car sequencing problem is a well-known scheduling problem which has applications in automotive assembly lines $([60]-[62])$. The assembly line has different stations which can process a number of cars with different options. Consequently, the cars must be scheduled in a sequence such that the capacity of each station is never exceeded. Papers [25]-[30] address car sequencing problem to minimize color changes, although it may be implied that minimizing color change could lead to better paint quality. Basically, three approaches are employed. The first method uses integer linear programming to model and solve the sequencing problem ([30]). The second one approaches the question as a constraint satisfaction problem $([25],[26])$. The third approach proposes an adaptation of the Ant Colony Optimization for the sequencing problem $([27],[28])$. All the approaches are actually involved in optimization algorithms. Typically, there are two deficiencies inside them. First, some optimization methods are employed to search the solution. However, there is a risk that a feasible solution can not be found. Second, optimization methods can not provide any implication of the sequencing problem. Neither can provide guidance to practical applications.

Similarly, re-sequencing at a color batching process in an automotive paint shop is analyzed in paper [63]. The re-sequencing problem with limited flexibility and sequence-dependent changeover costs is studied in papers [64] and 665. In these studies, the goals are to reduce set-up or changeover costs rather than improve quality. In addition, paper [66] investigates the location of sequencing point for multiple 
products automobile assembly systems. It claims that locating the sequencing point close to the final assembly provides more flexibility, to respond to defects or sequence change. However, it does not consider the issue of coupling between sequencing and quality.

As indicated above, the issue of quality improvement in a flexible manufacturing systems with batch productions has not been addressed in the current literature. In practice, improving performance is usually accomplished by identifying the bottleneck and improving its operation. Although most publications on bottleneck focus on improving productivity of a production system, their methods can still provide intellectual insight to quality bottleneck.

Paper 32] analyzes serial production lines consisting of machines and intermediate buffers where all machines follow the Bernoulli reliability machine model ([31]). The authors define the bottleneck machine as the one whose sensitivity (i.e., partial derivatives) of system's performance with respect to the machine's production rate in isolation is the largest. However, this definition suffers from the sensitivities involved which can not be either measured on-line or calculated off-line. Instead, a bottleneck identification rule is extended from two-machine line to identify the location of the bottleneck by analyzing frequencies of blockages and starvations which could be either measured or calculated.

Considering production lines with exponential machines, paper [33] defines the production rate bottleneck (PR-BN) as the machine which has the largest sensitivity on the system's throughput. Unfortunately, direct identification of PR-BNs using this definition is impossible because the derivatives of the production rate involved can 
not be measured or calculated on the factory floor. Therefore, bottleneck indicators are developed to seek the PR-BNs based on either practical data available on the factory floor or the data that can be calculated from the parameters of machines and buffers. Similar applications can be found in papers [34] and [35]. Furthermore, paper [36] investigates assembly systems with unreliable machines whose statistics are non-exponential. The bottleneck machine is defined as the sensitivity of system's throughput with respect to the machine's cycle time because of unknown production rate. Paper [37] defines bottlenecks for production lines with rework. Indicators as an indirect tool to identify bottlenecks are always used.

In addition to PR-BNs, paper [38] considers a pull serial production line and defines the due-time performance bottleneck (DTP-BN) where the due-time performance quantifies the level of customer demand satisfaction, i.e. the probability to ship a customer the required number of parts during a fixed time interval. Again, the direct definition of DTP-BN which is related to partial derivatives of due-time performance with respect to machine efficiencies is not applicable in practice. Therefore, bottleneck indicators are provided for identification of DTP-BNs in pull serial production lines by heuristically rather than analytically because of challenging mathematical complexity on partial derivatives.

\section{$2.3 \quad$ EEEF and Quality}

Due to huge energy cost and VOC emissions, research attention has been paid to improve energy efficiency and reduce emissions in automotive painting processes. Most

of the studies focus on renovation in painting processes. For example, paper [39] 
presents a summary of the vehicle assembly process and its energy use. Processspecific energy efficiency measures for painting systems are provided. Opportunities such as reducing air flow in painting booths, and heat recovery to reduce waste heat, are proposed to minimize energy usage. Paper [67] outlines the typical processes for vehicle painting, and identifies repetitive processes of painting and curing as the huge energy consumers. Effort has been made to decrease the number of processes in painting booths to reduce energy consumption. Paper [68] describes a process based cost model to estimate the economic costs in automotive painting. This model can be used for both reduction of VOC emissions and study of energy efficiency. The users can specify particular inputs and pre-assign factors in the model to evaluate the impact of process changes and alternative technologies on painting cost. Paper [69] investigates the major energy-consuming units in an assembly painting process. An equation is proposed to model the energy usage of each unit. In addition to energy consumption, the model can also evaluate associated environmental impacts and economic costs for automotive painting operations. Paper [70] studies environmental impact of different automotive paint materials, including solvent-based and power primers, water-based basecoat, and solvent-based and powder clearcoats based on life cycle analysis (LCA). Paper [71] proposes an energy, environmental and economic improvement assessment for automotive paint process. Papers [72] and [73] report that reducing over spray and smoothing cleaning process could lead to reduction of Carbon Dioxide emissions, and provides suggestions of using new type of paints and better booth design and booth management practice to achieve this goal.

In spite of such efforts, there is still a need to investigate how to achieve energy 
efficient painting through improved production operations. In other words, research effort is valuable to study how productivity and quality improvement can lead to reduction of energy consumption and atmospheric emissions. Since paint shop is typically the bottleneck of vehicle assembly plant, and the paint envelop largely determines the capacity of the whole assembly plant, and moreover, paint quality is one of the most significant measure in vehicle quality matrix, substantial effort has been devoted to improve productivity and quality in automotive paint shops. For example, papers [74] and [75] have introduced analytical models to evaluate the throughput in paint shops and procedures for continuous improvement. Case studies in paint shops are introduced to demonstrate the applicability of these methods. Designing repair and rework system to improve quality buy rate and its robustness in paint shops is presented by papers [76] and [22]. It is shown that selecting appropriate repair capacity can lead to improvement in quality and reduction of repaints. In addition, a case study to address the coupling between operation speed and quality in paint shop is introduced by paper [77].

It is also discovered by paper [78] that there is significant correlation between paint defects and emission levels. Superior environmental performance is associated with better quality. The plants with higher emissions levels typically have poorer associated quality. Similar observation is found by paper [79] that enhancing quality and efficiency also impacts environmental performance levels positively. Quality and environmental problems have many of the same causal factors. 


\subsection{Summary}

How to evaluate and improve quality in a flexible manufacturing system with batch productions has not been discussed in the current literature. Due to significant impact of flexibility on product quality, developing a method to predict or estimate quality is necessary and important. Quality improvement can be achieved by identifying bottleneck sequences and transitions (or product changes). Most publications on bottleneck focus on system throughput. In these studies, direct definitions based on partial derivatives are first provided and then bottleneck indicators are developed and applied using the data available on the factory floor. Such indicators could lead to identification of bottlenecks without complicated calculations. Similar to these studies, quality bottleneck with respect to product sequencing and transitions could also be defined and corresponding bottleneck indicators could be developed based on collected data. The identified bottleneck sequence or transition can be avoided or improved so that the product quality is improved.

In automotive paint shops, appropriate sequencing and batch policies can result in substantial quality improvement. Such quality improvement can also lead to fewer repaints, shorter flow time, less material usage, and therefore, less energy consumption and smaller impact to environment. Hence, developing optimal scheduling and control policies is necessary and important for energy efficient and environment friendly manufacturing in automotive paint shops.

Therefore, in spite of the above efforts, the impact of flexibility in terms of sequencing and batch policies on quality in a flexible manufacturing system with batch 
production has not been addressed. Analytical methods to evaluate quality performance in such systems, investigate the coupling between quality and flexibility, and identify the quality bottlenecks are of importance. Understanding the corresponding structural properties provides a foundation and guidance for managing quality improvement procedures. 


\section{CHAPTER 3}

\section{ANALYSIS OF QUALITY IN FLEXIBLE SYSTEMS}

In this chapter, an analytical model based on discrete Markov chain is presented. The quality evaluation formula in a simple case of two product types with batch size two is first derived and its monotonicity with respect to quality failure and repair probabilities is verified. In general case of multiple products multiple batch sizes, monotonic and non-monotonic properties on quality are analyzed, and asymptotic and oscillating properties are investigated to provide the necessary conditions when monotonicity still holds.

\subsection{Modeling based on Markov Chain}

Consider a flexible manufacturing system capable of producing different types of products. The following assumptions address the flexible production system, product types, sequence, and quality characteristics.

(1) The flexible system can process $n$ different types of products, denoted as 1,2 , $\ldots, n$. Each product type $i$ is processed in a batch with batch size $k_{i}, k_{i} \geq 1$.

(2) For a system with $n$ different part types, there are $(n-1)$ ! permutations of sequence, denoted as $s^{l}, l=1, \ldots,(n-1)$ !. The products flow into the system with sequence $s^{l}=\left\{s_{1}^{l}, s_{2}^{l}, \cdots, s_{n}^{l}\right\}$, where $s_{m}^{l}$ denotes the $m$-th product type in sequence $s^{l}$, and $m \in\{1,2, \cdots, n\}, s_{m}^{l} \in\{1,2, \cdots, n\}$. 
(3) For each sequence $s^{l}$, the flexible system will work on product type $s_{m}^{l}$ for $k_{s_{m}^{l}}$ parts before switching to product type $s_{m+1}^{l}$. It is assumed that product type $s_{1}^{l}$ is processed again after processing type $s_{n}^{l}$.

(4) The state of the flexible system is in good state $g_{s_{i}^{l}, j}$, or defective state $d_{s_{i}^{l}, j}$, $i=1, \ldots, n, j=1, \ldots, k_{s_{i}^{l}}$, if it is processing the $j$-th part in the batch of the product type $s_{i}^{l}$ with good quality, or with defects, respectively. Thus, there are $2 K, K=\sum_{i=1}^{n} k_{i}$, states in the system for a given sequence, defined by the quality status, product type processed and its position within a batch.

(5) When the system is in good state $g_{s_{i}^{l}, j}, s_{i}^{l}=1, \ldots, n, j=1, \ldots, k_{s_{i}^{l}}-1$, it has probabilities $\lambda_{s_{i}^{l}, s_{i}^{l}}$ to transit to defective state $d_{s_{i}^{l}, j+1}$, and $1-\lambda_{s_{i}^{l}, s_{i}^{l}}$ to good state $g_{s_{i}^{l}, j+1}$. Analogously, when the system is in defective state $d_{s_{i}^{l}, j}, s_{i}^{l}=1, \ldots, n$, $j=1, \ldots, k_{s_{i}^{l}}-1$, it can transit to good state $g_{s_{i}^{l}, j+1}$ with probability $\mu_{s_{i}^{l}, s_{i}^{l}}$, and to defective state $d_{s_{i}^{l}, j+1}$ with $1-\mu_{s_{i}^{l}, s_{i}^{l}}$.

(6) When the system is processing the last part within a batch and in good state $g_{s_{i}^{l}, k_{s_{i}^{l}}^{l}}, i=1, \ldots, n-1$, it has probabilities $\lambda_{s_{i+1}^{l}, s_{i}^{l}}$ and $1-\lambda_{s_{i+1}^{l}, s_{i}^{l}}$ to transit to states $d_{s_{i+1}^{l}, 1}$ and $g_{s_{i+1}^{l}, 1}$, respectively. Analogously, when the system is in defective state $d_{s_{i}^{l}, k_{s_{i}}}$, it has probabilities $\mu_{s_{i+1}^{l}, s_{i}^{l}}$ and $1-\mu_{s_{i+1}^{l}, s_{i}^{l}}$ to transit to states $g_{s_{i+1}^{l}, 1}$ and $d_{s_{i+1}^{l}, 1}$, respectively.

(7) When the system is in state $g_{s_{n}^{l}, k_{s_{n}^{l}}}$, it has probabilities $\lambda_{s_{1}^{l}, s_{n}^{l}}$ and $1-\lambda_{s_{1}^{l}, s_{n}^{l}}$ to transit to states $d_{s_{1}^{l}, 1}$ and $g_{s_{1}^{l}, 1}$, respectively. Analogously, when the system is in state $d_{s_{n}^{l}, k_{s_{n}^{l}}}$, it has probabilities $\mu_{s_{1}^{l}, s_{n}^{l}}$ and $1-\mu_{s_{1}^{l}, s_{n}^{l}}$ to transit to states $g_{s_{1}^{l}, 1}$ 
and $d_{s_{1}^{l}, 1}$, respectively. Without loss of generality, we assume all $0<\lambda_{i j}<1$, $0<\mu_{i j}<1, \forall i, j$.

Remark 3.1 Probabilities $\lambda_{s_{i}^{l}, s_{i}^{l}}, i=1, \ldots, n$, and $\lambda_{s_{j+1}^{l}}, s_{j}^{l}, j=1, \ldots, n-1$ and $\lambda_{s_{1}^{l}, s_{n}^{l}}$ are referred to as the quality failure probabilities. Similarly, $\mu_{s_{i}^{l}, s_{i}^{l}}$, $i=1, \ldots, n$, and $\mu_{s_{j+1}^{l}, s_{j}^{l}}, j=1, \ldots, n-1$ and $\mu_{s_{1}^{l}, s_{n}^{l}}$ are the quality repair probabilities. In addition, we denote $\lambda_{s_{i}^{l}, s_{i}^{l}}$ and $\mu_{s_{i}^{l}, s_{i}^{l}}$, as the quality failure and repair probabilities without product switch, and $\lambda_{s_{i}^{l}, s_{j}^{l}}$ and $\mu_{s_{i}^{l}, s_{j}^{l}}, i \neq j$, with product switch, respectively. Moreover, we define quality efficiencies with and without product switch as $e_{s_{i}^{l}, s_{j}^{l}}$ and $e_{s_{i}^{l}, s_{i}^{l}}$, respectively, where

$$
\begin{aligned}
e_{s_{i}^{l}, s_{j}^{l}} & =\frac{\mu_{s_{i}^{l}, s_{j}^{l}}}{\lambda_{s_{i}^{l}, s_{j}^{l}}+\mu_{s_{i}^{l}, s_{j}^{l}}}, i \neq j \\
e_{s_{i}^{l}, s_{i}^{l}} & =\frac{\mu_{s_{i}^{l}, s_{i}^{l}}}{\lambda_{s_{i}^{l}, s_{i}^{l}}+\mu_{s_{i}^{l}, s_{i}^{l}}} .
\end{aligned}
$$

Remark 3.2 Clearly, product change is not the only factor, and there exist many other issues, affecting quality in flexible manufacturing systems. For example, in automotive paint shops, in addition to color changes, dirts, paint mixture, humidity, etc., are also critical to paint quality. In this model, we focus on the impact of product change on quality, by assuming that the other factors may have equal influence on different products and have been embedded within the transition probabilities.

Denote $P\left(g_{s_{i}^{l}, j}\right)$ and $P\left(d_{s_{i}^{l}, j}\right), i=1, \ldots, n, j=1, \ldots, k_{s_{i}^{l}}$, as the probabilities that the system is in state $g_{s_{i}^{l}, j}$ or $d_{s_{i}^{l}, j}$ (i.e., producing a good or a defective job for the $j$-th part in the batch of product type $s_{i}^{l}$ ), respectively. Then, the overall 
quality performance of the flexible system for a given sequence $s^{l}$, i.e., the probability to produce a good (or, a defective) part in batch production, is defined as $P\left(g_{b t}^{l}\right)$ (respectively, $P\left(d_{b t}^{l}\right)$ ), and is calculated from

$$
\begin{aligned}
& P\left(g_{b t}^{l}\right)=\sum_{s_{i}^{l}=1}^{n} \sum_{j=1}^{k_{s_{i}^{l}}} P\left(g_{s_{i}^{l}, j}\right), \\
& P\left(d_{b t}^{l}\right)=\sum_{s_{i}^{l}=1}^{n} \sum_{j=1}^{k_{s_{i}^{l}}^{l}} P\left(d_{s_{i}^{l}, j}\right) .
\end{aligned}
$$

In the subsequent sections, we begin the study with the case of two product types with batch size two. Then we will extend to more general cases. Note that the quality evaluation below is focused on the sequence $s^{l}=\{1,2, \cdots, n\}$, and the sequence index $l$ is ignored for simplicity.

\subsection{Two Product Types with Batch Size Two}

A quality evaluation formula in this simple case is derived, and then its properties are discussed.

\subsubsection{Analytical expressions}

Consider that there are two types of products, 1 and 2, and each has batch size two. A state transition diagram is illustrated in Figure 3.1.

Based on the state transition diagram, we have

$$
\begin{aligned}
P\left(g_{11}, t+1\right) & =P\left(g_{11}, t+1 \mid d_{22}, t\right) P\left(d_{22}, t\right)+P\left(g_{11}, t+1 \mid g_{22}, t\right) P\left(g_{22}, t\right) \\
& =\mu_{12} P\left(d_{22}, t\right)+\left(1-\lambda_{12}\right) P\left(g_{22}, t\right) .
\end{aligned}
$$

In terms of the steady state, let

$$
\lim _{t \rightarrow \infty} P\left(g_{11}, t\right)=: P\left(g_{11}\right),
$$




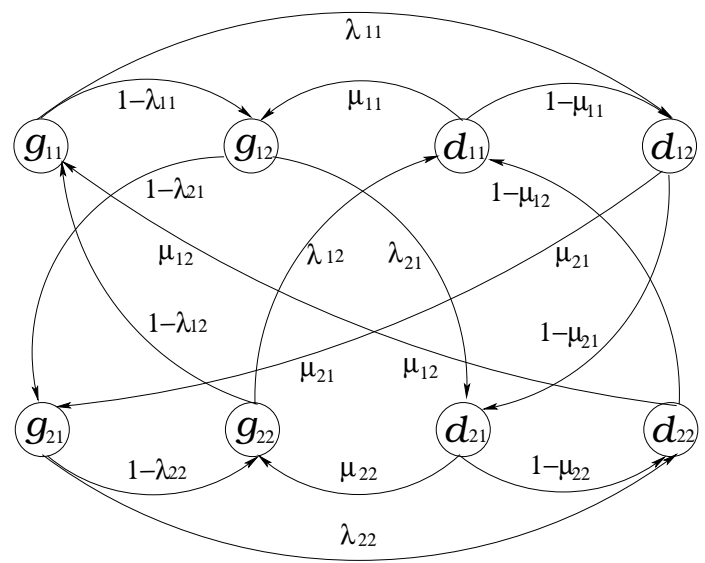

Figure 3.1: State transition diagram of two-product type case with batch size two

we obtain

$$
P\left(g_{11}\right)=\mu_{12} P\left(d_{22}\right)+\left(1-\lambda_{12}\right) P\left(g_{22}\right) .
$$

Similarly, we derive

$$
\begin{aligned}
& P\left(g_{12}\right)=\mu_{11} P\left(d_{11}\right)+\left(1-\lambda_{11}\right) P\left(g_{11}\right), \\
& P\left(g_{21}\right)=\mu_{21} P\left(d_{12}\right)+\left(1-\lambda_{21}\right) P\left(g_{12}\right), \\
& P\left(g_{22}\right)=\mu_{22} P\left(d_{21}\right)+\left(1-\lambda_{22}\right) P\left(g_{21}\right), \\
& P\left(d_{11}\right)=\left(1-\mu_{12}\right) P\left(d_{22}\right)+\lambda_{12} P\left(g_{22}\right), \\
& P\left(d_{12}\right)=\left(1-\mu_{11}\right) P\left(d_{11}\right)+\lambda_{11} P\left(g_{11}\right), \\
& P\left(d_{21}\right)=\left(1-\mu_{21}\right) P\left(d_{12}\right)+\lambda_{21} P\left(g_{12}\right), \\
& P\left(d_{22}\right)=\left(1-\mu_{22}\right) P\left(d_{21}\right)+\lambda_{22} P\left(g_{21}\right) .
\end{aligned}
$$

In addition, the total probability is equal to 1 ,

$$
P\left(g_{11}\right)+P\left(g_{12}\right)+P\left(g_{21}\right)+P\left(g_{22}\right)+P\left(d_{11}\right)+P\left(d_{12}\right)+P\left(d_{21}\right)+P\left(d_{22}\right)=1 .
$$

Then, using these equations, we can derive the probability of good parts as follows: 
Theorem 3.1 Under assumptions (1)-(7) with $n=2$ and $k_{1}=k_{2}=2$, the probability of producing a good part can be calculated by

$$
P(g)=\frac{\mathcal{A}+\mathcal{B}+\mathcal{C}+\mathcal{D}}{4(1-\mathcal{F})}
$$

where

$$
\begin{aligned}
& \mathcal{A}=\left[\mu_{21}+\mu_{11}\left(1-\lambda_{21}-\mu_{21}\right)\right]\left[1+\left(1-\lambda_{22}-\mu_{22}\right)\left(1-\lambda_{12}-\mu_{12}\right)\right], \\
& \mathcal{B}=\left[\mu_{11}+\mu_{12}\left(1-\lambda_{11}-\mu_{11}\right)\right]\left[1+\left(1-\lambda_{22}-\mu_{22}\right)\left(1-\lambda_{21}-\mu_{21}\right)\right], \\
& \mathcal{C}=\left[\mu_{22}+\mu_{21}\left(1-\lambda_{22}-\mu_{22}\right)\right]\left[1+\left(1-\lambda_{12}-\mu_{12}\right)\left(1-\lambda_{11}-\mu_{11}\right)\right], \\
& \mathcal{D}=\left[\mu_{12}+\mu_{22}\left(1-\lambda_{12}-\mu_{12}\right)\right]\left[1+\left(1-\lambda_{11}-\mu_{11}\right)\left(1-\lambda_{12}-\mu_{12}\right)\right], \\
& \mathcal{F}=\left(1-\lambda_{21}-\mu_{21}\right)\left(1-\lambda_{22}-\mu_{22}\right)\left(1-\lambda_{11}-\mu_{11}\right)\left(1-\lambda_{12}-\mu_{12}\right) .
\end{aligned}
$$

Proof: See Appendix A.

Next, we consider the case where the transition probabilities are identical for both products, denoted as the equal products case, i.e.,

$$
\mu_{12}=\mu_{21}, \quad \lambda_{12}=\lambda_{21}, \quad \mu_{11}=\mu_{22}, \quad \lambda_{11}=\lambda_{22} .
$$

Then, equation 3.14 can be simplified as follows:

Corollary 3.1 Under assumptions (1)-(7) with $n=2, k_{1}=k_{2}=2$ in the equal products case, the probability of producing a good part can be calculated as

$$
P(g)=\frac{\mu_{11}\left(2-\lambda_{21}-\mu_{21}\right)+\mu_{21}\left(2-\lambda_{11}-\mu_{11}\right)}{2\left[1-\left(1-\lambda_{11}-\mu_{11}\right)\left(1-\lambda_{21}-\mu_{21}\right)\right]} .
$$

Proof: See Appendix A.

Using this expression, we can compare the quality performance of batch policy with other scheduling policies in flexible manufacturing systems. For simplicity, we will limit our discussions to the equal products case. 


\subsubsection{Monotonicity}

Similar to the results obtained in [21], the product quality will be monotonically increasing with respect to quality repair probabilities and decreasing to quality failure probabilities.

Corollary 3.2 Under assumptions (1)-(7) with $n=2, k_{1}=k_{2}=2$ in the equal products case, the probability of producing a good part $P(g)$ is monotonically

- decreasing with respect to $\lambda_{11}$ and $\lambda_{21}$;

- increasing with respect to $\mu_{11}$ and $\mu_{21}$.

Proof: See Appendix A.

\subsubsection{Comparisons with other sequencing policies}

In this subsection, we compare the quality performance using batch policy with strict sequencing and random mixing policies. In strict sequencing policy, product type changes at every cycle. In other words, two types of products are sequenced as $1,2,1,2,1, \ldots$ In random mixing policy, product types are coming in a random order with uniformly distributions.

\section{Comparison with strict sequencing policy}

First, introduce $e_{11}$ and $e_{21}$ as the quality efficiency without product switch and quality efficiency with product switch, respectively, where

$$
e_{11}=\frac{\mu_{11}}{\lambda_{11}+\mu_{11}}, \quad e_{21}=\frac{\mu_{21}}{\lambda_{21}+\mu_{21}}
$$

Then, denote the probability of good parts for batch and strict sequencing policy as $P\left(g_{b t}\right)$ and $P\left(g_{s s}\right)$, respectively. 
Corollary 3.3 Under assumptions (1)-(7) with $n=2, k_{1}=k_{2}=2$ in the equal products case, the following statements hold:

$$
\begin{aligned}
& \text { (1) } P\left(g_{b t}\right)>P\left(g_{s s}\right) \quad \text { if } e_{11}>e_{21} \text {, } \\
& \text { (2) } P\left(g_{b t}\right)<P\left(g_{s s}\right) \quad \text { if } e_{11}<e_{21} \text {. }
\end{aligned}
$$

Proof: See Appendix A.

This Corollary indicates that when the quality efficiency is higher within same products, using batch can achieve better quality performance compared to strictly sequencing the products.

\section{Comparison with random mixing policy}

Let $P\left(g_{r}\right)$ denote the probability of good parts using random mixing policy. We have

Corollary 3.4 Under assumptions (1)-(7) with $n=2$ and $k_{1}=k_{2}=2$ in the equal products case, if $e_{11}>e_{21}$ and $\mu_{11}$ is sufficiently larger than $\mu_{21}$ so that $\mu_{11}>$ $\frac{e_{11}}{e_{21}} \mu_{21}$, then

$$
P\left(g_{b t}\right)>P\left(g_{r}\right)
$$

Proof: See Appendix A.

Therefore, using batch is better than randomly mixing the two product types in terms of quality performance if $e_{11}>e_{21}$ and the repair probability without product switch is sufficiently higher than the repair probability with product switch. Clearly, when quality efficiency is good within a batch, and the system can quickly recover from processing defective jobs, then batch policy is a better choice. 
As it has been shown in [21], random mixing policy is better in quality than strict sequencing policy when $e_{11}>e_{21}$, we can conclude that, in this case, batch production is the best option to obtain high quality performance, while strict sequencing is the worst choice, and random mixing falls in the middle.

\subsection{General Multiple Products Multiple Batch Sizes Case}

A quality evaluation formula in general case is derived, and then its properties are discussed.

\subsubsection{General formulas}

For $n$ products, each has $k_{i}$ parts in a batch, $i=1, \ldots, n$, from assumptions (1)-(7) and following the similar procedure in Section 3.2, we have

$$
\begin{aligned}
& P\left(g_{i j}\right)=\mu_{i i} P\left(d_{i, j-1}\right)+\left(1-\lambda_{i i}\right) P\left(g_{i, j-1}\right), \\
& P\left(d_{i j}\right)=\left(1-\mu_{i i}\right) P\left(d_{i, j-1}\right)+\lambda_{i i} P\left(g_{i, j-1}\right), i=1, \ldots, n, j=2, \ldots, k_{i}, \\
& P\left(g_{i 1}\right)=\mu_{i, i-1} P\left(d_{i-1, k_{i-1}}\right)+\left(1-\lambda_{i, i-1}\right) P\left(g_{i-1, k_{i-1}}\right), \\
& P\left(d_{i 1}\right)=\left(1-\mu_{i, i-1}\right) P\left(d_{i-1, k_{i-1}}\right)+\lambda_{i, i-1} P\left(g_{i-1, k_{i-1}}\right), i=2, \ldots, n, \\
& P\left(g_{11}\right)=\mu_{1 n} P\left(d_{n, k_{n}}\right)+\left(1-\lambda_{1 n}\right) P\left(g_{n, k_{n}}\right), \\
& P\left(d_{11}\right)=\left(1-\mu_{1 n}\right) P\left(d_{n, k_{n}}\right)+\lambda_{1 n} P\left(g_{n, k_{n}}\right),
\end{aligned}
$$

and

$$
\sum_{i=1}^{n} \sum_{j=1}^{k_{i}}\left[P\left(g_{i j}\right)+P\left(d_{i j}\right)\right]=1 .
$$

In matrix form, we obtain

$$
A X=B
$$


where

$$
\begin{gathered}
A=\left(\begin{array}{cccccccccc}
1 & \cdots & 0 & \lambda_{1 n}-1 & 0 & 0 & \cdots & 0 & 0 & -\mu_{1 n} \\
\lambda_{11}-1 & \cdots & 0 & 0 & -\mu_{11} & 0 & \cdots & 0 & 0 & 0 \\
\cdots & \cdots & \cdots & \cdots & \cdots & \cdots & \cdots & \cdots & \cdots & \cdots \\
0 & \cdots & \lambda_{n n}-1 & 1 & 0 & 0 & \cdots & 0 & -\mu_{n n} & 0 \\
0 & \cdots & 0 & -\lambda_{1 n} & 1 & 0 & \cdots & 0 & 0 & \mu_{1 n}-1 \\
-\lambda_{11} & \cdots & 0 & 0 & \mu_{11}-1 & 1 & \cdots & 0 & 0 & 0 \\
0 & \cdots & 0 & 0 & 0 & \mu_{11}-1 & \cdots & 0 & 0 & 0 \\
\cdots & \cdots & \cdots & \cdots & \cdots & \cdots & \cdots & \cdots & \cdots & \cdots \\
0 & \cdots & 0 & 0 & 0 & 0 & \cdots & \mu_{n n}-1 & 1 & 0 \\
1 & \cdots & 1 & 1 & 1 & 1 & \cdots & 1 & 1 & 1
\end{array}\right), \\
B \\
\quad=[0, \ldots, 0,1]^{T}, \\
X \\
\quad=\left[P\left(g_{11}\right), \ldots, \ldots, P\left(g_{n, k_{n}}\right), P\left(d_{11}\right), \ldots, P\left(d_{n, k_{n}}\right)\right]^{T} .
\end{gathered}
$$

Therefore, we can obtain the probability of good parts $P(g)$ as follows:

Theorem 3.2 Under assumptions (1)-(7) with multiple products multiple batch sizes, the probability of good parts $P(g)$ is calculated by

$$
P(g)=\sum_{i=1}^{n} \sum_{j=1}^{k_{i}} P\left(g_{i j}\right)=\sum_{i=1}^{K} x_{i}
$$

where $K=\sum_{i=1}^{n} k_{i}$, and $x_{i j}$ is solved from

$$
X=A^{-1} B
$$

and $A, B, X$ are defined in Equations (3.30)-(3.32).

Remark 3.3 Since a unique solution and the steady state exist for an irreducible Markov chain with finite number of states, the inverse of transition matrix $A$ exists.

This formula provides a method to evaluate the quality performance of a flexible manufacturing system with batch productions and enables us to investigate systemtheoretic properties, design principles, scheduling and improvement policies. 
When $n=k_{1}=k_{2}=2$, we obtain matrix $A_{2}$ (where subscript '2' denotes $n=2$ ) in Equation 3.35, and solving Equation (3.34 will lead to the same solution as in Equation (3.14.

$$
A_{2}=\left(\begin{array}{cccccccc}
1 & 0 & 0 & \lambda_{12}-1 & 0 & 0 & 0 & -\mu_{12} \\
\lambda_{11}-1 & 1 & 0 & 0 & -\mu_{11} & 0 & 0 & 0 \\
0 & \lambda_{21}-1 & 1 & 0 & 0 & -\mu_{21} & 0 & 0 \\
0 & 0 & \lambda_{22}-1 & 1 & 0 & 0 & -\mu_{22} & 0 \\
0 & 0 & 0 & -\lambda_{12} & 1 & 0 & 0 & \mu_{12}-1 \\
-\lambda_{11} & 0 & 0 & 0 & \mu_{11}-1 & 1 & 0 & 0 \\
0 & -\lambda_{21} & 0 & 0 & 0 & \mu_{21}-1 & 1 & 0 \\
1 & 1 & 1 & 1 & 1 & 1 & 1 & 1
\end{array}\right)
$$

In the case of equal products, the transition probabilities and batch sizes must satisfy

$$
\begin{aligned}
& \lambda_{i i}=\lambda_{11}, \mu_{i i}=\mu_{11}, i=1, \ldots, n, \\
& \lambda_{1 n}=\lambda_{i+1, i}=\lambda_{21}, \mu_{1 n}=\mu_{i+1, i}=\mu_{21}, i=1, \ldots, n-1, \\
& k_{i}=k_{1}=k, i=1, \ldots, n .
\end{aligned}
$$

Corollary 3.5 Under assumptions (1)-(7) with multiple products multiple batch sizes in the equal products case, the probability of good parts can be calculated as

$$
P(g)=\frac{\mu_{11}}{\lambda_{11}+\mu_{11}}+\frac{\left(\lambda_{11} \mu_{21}-\lambda_{21} \mu_{11}\right)\left[1-\left(1-\lambda_{11}-\mu_{11}\right)^{k}\right]}{k\left(\lambda_{11}+\mu_{11}\right)^{2}\left[1-\left(1-\lambda_{21}-\mu_{21}\right)\left(1-\lambda_{11}-\mu_{11}\right)^{k-1}\right]} .
$$

Proof: See Appendix A.

Clearly, when $k=1, P(g)=e_{21}$. When $k=2$, Equation (3.36) reduces to Equation 3.20. 
Remark 3.4 Note that Equation 3.36 is independent of the number of product types, $n$. The rationale behind this is that since all products are identical, transitions from product type $i$ to type $i+1$ are same for all $i, i=1, \ldots, n-1$, and is also same as transition from product type $n$ to type 1 . Therefore, it is equivalent to that there are only two product types, 1 and 2 . Therefore, the quality performance only depends on the batch size $k$. However, when $\lambda_{i j}$ and $\mu_{i j}$ are not same, and $k_{i}$ is not same, the number of products will play a role.

Remark 3.5 The introduction of equal products case not only simplifies the analysis, but also has wide practical applications. For example, in body shop, vehicles with different sizes can be processed on the same line. The transition probabilities among different sizes of vehicles, $\lambda_{i j}$ 's (or $\mu_{i j}$ 's), are typically similar, and probabilities

within each style, $\lambda_{i i}$ 's (or $\mu_{i i}$ 's), are also close to each other. Similar scenario can be observed in flexible machining lines, where the transition probabilities between batches are determined by the location errors of the flexible fixtures, independent of product types, and the transition probabilities within batches are dominated by tooling errors, which are similar for all products. In these cases, analysis of equal products case can be applicable.

\subsubsection{Bernoulli quality reliability case}

Here we consider a special case $\lambda_{11}+\mu_{11}=\lambda_{21}+\mu_{21}=1$, i.e., the system quality reliability follows a Bernoulli distribution. In this case, repair probabilities $\mu_{i 1}, i=$ 1,2 , define the probabilities to produce a good part without or with product switch. 
Then, we can simplify the analysis to

$$
P(g)=\mu_{11}+\frac{\mu_{21}-\mu_{11}}{k} .
$$

Clearly, when $\mu_{11}>\mu_{21}$, increasing batch size will lead to better quality performance, which agrees with our intuition since quality efficiency without switch is larger than that with switch. However, when $\mu_{21}>\mu_{11}$, i.e., switching products has better quality efficiency, negative effect will appear if larger batch is implemented. Therefore, smaller batches are preferred in this scenario. An illustration of both scenarios is presented in Figure 3.2 .

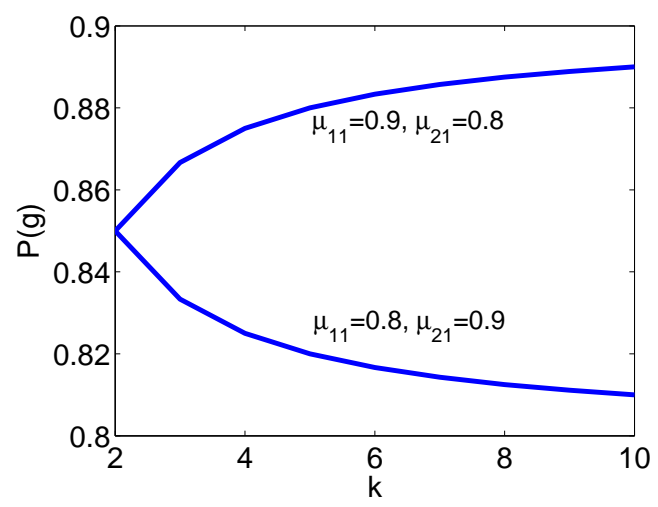

Figure 3.2: Product quality as a function of batch size in Bernoulli case

\subsubsection{Monotonic and non-monotonic properties with respect to quality failure and repair probabilities}

Intuitively, monotonic properties are often expected. For example, we may expect that the quality can be improved if quality failure probability is decreased, or repair probability is increased, or batch size is larger. If such properties do not hold, continuous improvement effort based on monotonic intuition may not be effective. Therefore, obtaining the knowledge of these properties could help identify the directions for continuous improvement to achieve better quality. 
For monotonicity with respect to $\lambda_{21}$ and $\mu_{21}$, we can show that such property holds in equal products case.

Proposition 3.1 Under assumption (1)-(7) in the equal products case, the probability of good parts, $P(g)$, is monotonically

- decreasing with respect to $\lambda_{21}$, and

- increasing with respect to $\mu_{21}$.

Proof: See Appendix A.

Unexpectedly, experiments suggest that monotonic property with respect to $\lambda_{11}$ and $\mu_{11}$ does not hold all the time. Numerical investigation indicates that in most cases, $P(g)$ will decrease or increase with respect to $\lambda_{11}$ and $\mu_{11}$, respectively, i.e., $\frac{\partial P(g)}{\partial \lambda_{11}}<0$ and $\frac{\partial P(g)}{\partial \mu_{11}}>0$. But in some extreme cases, opposite results may be observed. Table 3.1 and 3.2 present two examples of these counter-intuitive results.

Table 3.1: Non-monotonicity of $P(g)$ with respect to $\lambda_{11}$

\begin{tabular}{|c|c|c|c|c|c|c|}
\hline$k$ & $\lambda_{11}$ & $\lambda_{21}$ & $\mu_{11}$ & $\mu_{21}$ & $\frac{\partial P(g)}{\partial \lambda_{11}}$ & $P(g)$ \\
\hline 5 & 0.9200 & 0.0100 & 0.9900 & 0.4900 & -0.0044 & 0.5781 \\
\hline 5 & 0.9300 & 0.0100 & 0.9900 & 0.4900 & 0.0217 & 0.5781 \\
\hline 5 & 0.9400 & 0.0100 & 0.9900 & 0.4900 & 0.0506 & 0.5785 \\
\hline
\end{tabular}

Table 3.2: Non-monotonicity of $P(g)$ with respect to $\mu_{11}$

\begin{tabular}{|c|c|c|c|c|c|c|}
\hline$k$ & $\lambda_{11}$ & $\lambda_{21}$ & $\mu_{11}$ & $\mu_{21}$ & $\frac{\partial P(g)}{\partial \mu_{11}}$ & $P(g)$ \\
\hline 7 & 0.9900 & 0.6000 & 0.9600 & 0.0400 & 0.0092 & 0.4455 \\
\hline 7 & 0.9900 & 0.6000 & 0.9700 & 0.0400 & -0.0210 & 0.4457 \\
\hline 7 & 0.9900 & 0.6000 & 0.9800 & 0.0400 & -0.0552 & 0.4456 \\
\hline
\end{tabular}

It can be seen from these tables that these non-monotonic cases only occur when failure and repair probabilities without switch are typically large (approaching 1), 
which may seldom happen in real systems. In most practical situations, monotonicity can still be counted on.

\subsubsection{Asymptotic properties}

With different combinations of $\lambda_{i 1}$ and $\mu_{i 1}, i=1,2$, we consider several scenarios where $\lambda_{i 1}$ or $\mu_{i 1}$ approach 0 or 1 .

- Case 1: $\lambda_{11} \rightarrow 0, \mu_{11} \rightarrow 1$.

In this case, if the first job in a batch is in good quality, it probably will keep good quality for remaining jobs in the batch since $\lambda_{11} \rightarrow 0$. If the first one is defective, then it most likely changes to good quality states from the second one due to $\mu_{11} \rightarrow 1$. Thus, the second to last jobs in a batch are typically good. This implies $P\left(g_{i j}\right) \rightarrow 1, i=1, \ldots, n, j=2, \ldots, k$. An illustration of this observation is shown in Figure 3.3. In addition, we can show that

$$
\lim _{\lambda_{11} \rightarrow 0, \mu_{11} \rightarrow 1} P(g)=1-\frac{\lambda_{21}}{k} .
$$

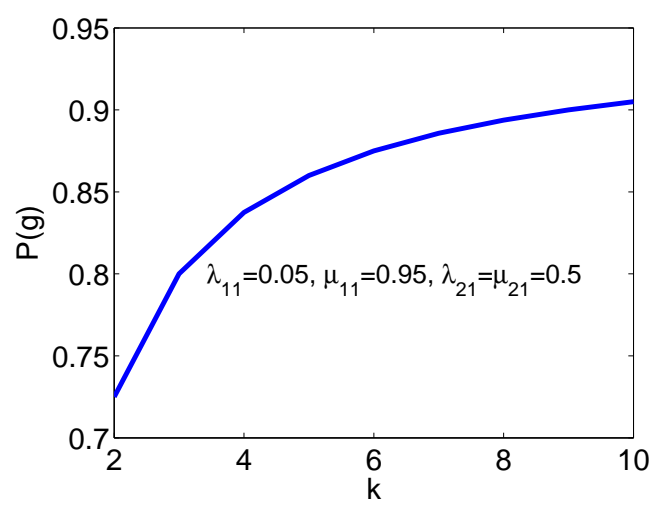

Figure 3.3: Asymptotic behavior: Case 1

When $\lambda_{21} \rightarrow 1$, it implies the product switch makes the first job in a batch is 
more likely to be defective. Therefore, $P(g) \rightarrow 1-1 / k$. If $\lambda_{21} \rightarrow 0$, it follows that usually all jobs are kept in good quality, i.e., $P(g) \rightarrow 1$.

- Case 2: $\lambda_{11} \rightarrow 1, \mu_{11} \rightarrow 0$.

This can be viewed as a dual (or an opposite) case of the first one. If the first job in a batch is in good quality, it probably will change to poor quality from the second job in the batch and keep it since $\lambda_{11} \rightarrow 1$. If the first one is defective, then it most likely keep poor quality due to $\mu_{11} \rightarrow 0$. Thus, the second to last jobs in a batch are typically defective. This leads to $P\left(d_{i j}\right) \rightarrow 1, i=1, \ldots, n$, $j=2, \ldots, k$. This phenomenon is illustrated in Figure 3.4 . we also prove that

$$
\lim _{\lambda_{11} \rightarrow 1, \mu_{11} \rightarrow 0} P(g)=\frac{\mu_{21}}{k} .
$$

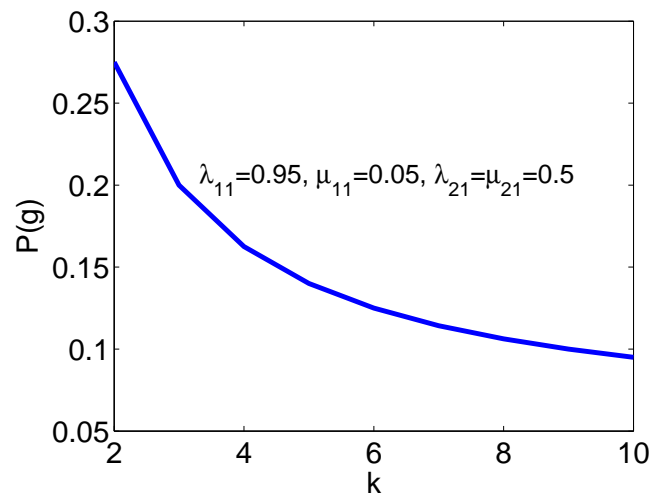

Figure 3.4: Asymptotic behavior: Case 2

Similar to the first case, $\mu_{21} \rightarrow 1$ implies product switch most likely leads to good quality in the first job in a batch. Hence, $P(g) \rightarrow 1 / k$. While $\mu_{21} \rightarrow 0$ implies that quite often all jobs are in defective states even with product switch, i.e., $P(g) \rightarrow 0$. 
In the above two cases, monotonic properties have been observed. Counter intuitively, monotonicity may not hold all the time.

- Case 3: $\lambda_{11} \rightarrow 1, \mu_{11} \rightarrow 1$.

- Case 3.1: $\lambda_{21} \rightarrow 0, \mu_{21} \rightarrow 1$.

In this case, if the last job in previous batch is good in quality, then due to $\lambda_{21} \rightarrow 0$, the first job in the current batch will stay in good quality; on the other hand, if the last job in previous batch is defective, then the first job in current batch will change to good quality due to $\mu_{21} \rightarrow 1$. Therefore, in either cases, first job is in good quality. Since $\lambda_{11} \rightarrow 1$, we have the odd number ones approach good quality and even ones defective. As a result, for even batch size, we obtain equal numbers of good and bad quality jobs; while for odd one, an additional good quality job is obtained compared to bad quality ones within a batch. In other words,

$$
\begin{aligned}
\lim _{\substack{\lambda_{11} \rightarrow 1, \lambda_{21} \rightarrow 0 \\
\mu_{11} \rightarrow 1, \mu_{21} \rightarrow 1}} P(g) & =\frac{1-(-1)^{k}}{4 k}+\frac{1}{2} \\
& = \begin{cases}\frac{1}{2 k}+\frac{1}{2} & k \text { odd } \\
\frac{1}{2} & k \text { even }\end{cases}
\end{aligned}
$$

- Case 3.2: $\lambda_{21} \rightarrow 1, \mu_{21} \rightarrow 0$.

Following the similar arguments as in Case 3.1, the odd number jobs are defective and even numbers are in good quality, which implies equal numbers of good and bad quality jobs for even batch size, and for odd batch 
size, one additional bad quality job is obtained. Thus,

$$
\begin{aligned}
\lim _{\substack{\lambda_{11} \rightarrow 1, \lambda_{21} \rightarrow 1 \\
\mu_{11} \rightarrow 1, \mu_{21} \rightarrow 0}} P(g) & =-\frac{1-(-1)^{k}}{4 k}+\frac{1}{2} \\
& = \begin{cases}-\frac{1}{2 k}+\frac{1}{2} & k \text { odd }, \\
\frac{1}{2} & k \text { even } .\end{cases}
\end{aligned}
$$

Illustrations of Cases 3.1 and 3.2 are presented in Figure 3.5. Clearly, decreasing or increasing oscillated behaviors have been observed in these two cases, respectively. It also indicates that when jobs are more likely to be in good quality after switch (i.e., $\lambda_{21} \rightarrow 0, \mu_{21} \rightarrow 1$ ), it will oscillate above 0.5 , and it is good to keep odd number of batch size. Otherwise, it oscillates below 0.5, and even numbered batch size is preferred.

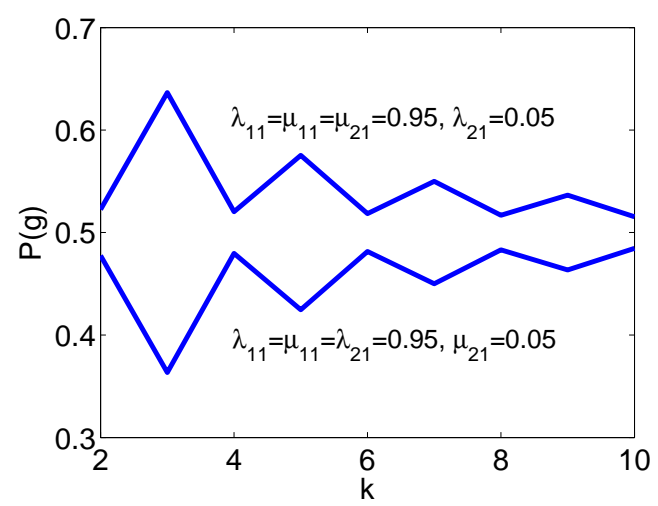

Figure 3.5: Asymptotic behavior: Cases 3.1 and 3.2

- Case 4: $\lambda_{11} \rightarrow 0, \mu_{11} \rightarrow 0$.

$-\lambda_{21} \rightarrow 0, \mu_{21} \rightarrow 0$. Then $P(g)$ is approaching 0 or 1 depending on the quality of the 1st part.

$-\lambda_{21} \rightarrow 1, \mu_{21} \rightarrow 1$. The system produces all good or all defective batches alternatively. Then, $P(g)=\frac{1}{2}$. 
$-\lambda_{21} \rightarrow 1, \mu_{21} \rightarrow 0$. It follows that the state will keep being defective after product switch, i.e., $P(g) \rightarrow 0$.

$-\lambda_{21} \rightarrow 0, \mu_{21} \rightarrow 1$. Opposite situation occurs, $P(g) \rightarrow 1$.

Intuitively we may assume that increasing batch size will improve quality. How-

ever, the asymptotic results described above (Case 3) suggest that oscillating behavior exists with respect to batch size $k$. Thus, we need to understand when oscillation may occur and under what condition monotonicity still holds, so that we can provide practical guidance for operation management. The next subsection is devoted to investigation of this phenomenon.

\subsubsection{Oscillating properties with respect to batch size}

Let $P_{k}(g)$ denote the probability of producing a good quality part when batch size is $k$. Then, we obtain

$$
\begin{aligned}
P_{k}(g) & =\frac{\mu_{11}}{\lambda_{11}+\mu_{11}}+\frac{\left(\lambda_{11} \mu_{21}-\lambda_{21} \mu_{11}\right)\left[1-\left(1-\lambda_{11}-\mu_{11}\right)^{k}\right]}{k\left(\lambda_{11}+\mu_{11}\right)^{2}\left[1-\left(1-\lambda_{21}-\mu_{21}\right)\left(1-\lambda_{11}-\mu_{11}\right)^{k-1}\right]} \\
& =\frac{\mu_{11}}{\lambda_{11}+\mu_{11}}+\frac{\left(\lambda_{21}+\mu_{21}\right)\left(e_{21}-e_{11}\right)\left(1-a^{k}\right)}{k\left(\lambda_{11}+\mu_{11}\right)\left(1-b a^{k-1}\right)} \\
& =\frac{\mu_{11}}{\lambda_{11}+\mu_{11}}+\frac{\left(\lambda_{21}+\mu_{21}\right)\left(e_{21}-e_{11}\right)}{\lambda_{11}+\mu_{11}} D_{k} .
\end{aligned}
$$

where

$$
\begin{aligned}
a & =1-\lambda_{11}-\mu_{11}, \\
b & =1-\lambda_{21}-\mu_{21}, \\
D_{k} & =\frac{1-a^{k}}{k\left(1-b a^{k-1}\right)} .
\end{aligned}
$$




\section{Increasing batch size by 1}

Consider

$$
P_{k+1}(g)-P_{k}(g)=\frac{\left(\lambda_{21}+\mu_{21}\right)\left(e_{21}-e_{11}\right)}{\lambda_{11}+\mu_{11}}\left(D_{k+1}-D_{k}\right) .
$$

Proposition 3.2 Under assumption (1)-(7) in the equal products case,

- $P_{2 j+2}(g)<P_{2 j+1}(g), j=0,1,2, \ldots$, i.e., monotonically decreasing with respect to odd batch size, if $e_{11}<e_{21}$;

- $P_{2 j+2}(g)>P_{2 j+1}(g), j=0,1,2, \ldots$, i.e., monotonically increasing with respect to odd batch size, if $e_{11}>e_{21}$.

Proof: See Appendix A.

Proposition 3.2 suggests that $D_{k+1}$ is always smaller than $D_{k}$ when $k$ is odd so that increasing batch size by 1 will lead to degradation of product quality when $e_{11}<e_{21}$, which agrees with our intuition since $e_{11}<e_{21}$ implies product switch will improve quality, and therefore smaller batch is preferred. Analogously, increasing batch size by 1 can improve quality when $e_{11}>e_{21}$. An illustration of the fact that $D_{2 j+2}<D_{2 j+1}$ is shown in Figure 3.6, where we select all possible values of $\lambda_{i 1}$ and $\mu_{i 1}$ between 0.01 and 0.99 with a step size 0.01 for different batch size $k$.

However, such property may not hold when $k$ is even. As it is shown in Figure 3.7 that $D_{k+1}-D_{k}$ can be positive or negative when $k$ is even, which implies that oscillating behavior may occur when the even batch size is increased. Clearly, there exists a boundary condition that $D_{k+1}$ may be greater than $D_{k}$ when $k$ is even. From 


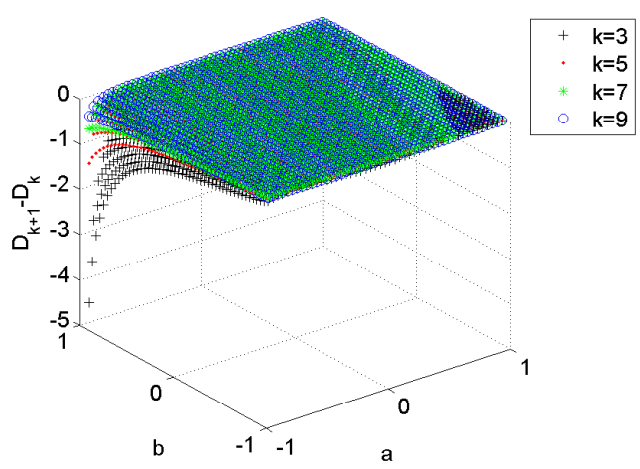

Figure 3.6: $D_{k+1}-D_{k}$ vs $a$ and $b$ with respect to different odd batch size $k$

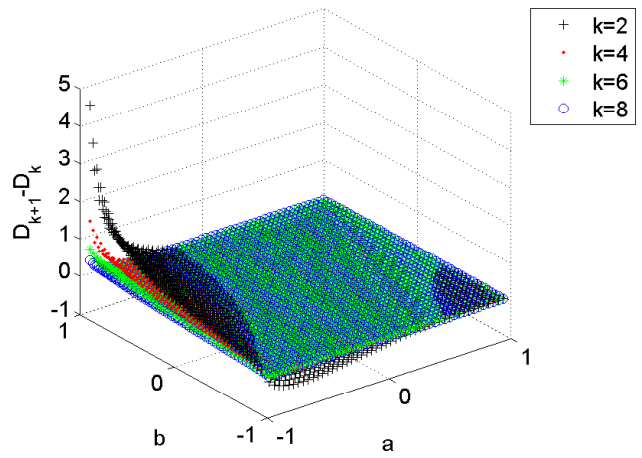

Figure 3.7: $D_{k+1}-D_{k}$ vs $a$ and $b$ with respect to different odd batch size $k$

Equation 3.38 , it can be shown that $D_{2 j+1}=D_{2 j}$ implies that

$$
\frac{1-a^{2 j+1}}{(2 j+1)\left(1-b a^{2 j}\right)}=\frac{1-a^{2 j}}{2 j\left(1-b a^{2 j-1}\right)}, j=0,1,2, \ldots .
$$

After some algebraic manipulation, we obtain the boundary condition

$$
b=\frac{1-a^{2 j}-2 j a^{2 j}(1-a)}{a^{2 j}\left(1-a^{2 j}\right)-2 j a^{2 j-1}(1-a)}, j=0,1,2, \ldots
$$

However, such formula does not provide a clear indication on what kind of parameters $\lambda_{i 1}, \mu_{i 1}, i=1,2$, leading to oscillation. Therefore, we calculate such boundaries numerically and plot on an $a-b$ plane for different even batch size $k$ (Figure 3.8). As one can see, when $a$ and $b$ are allocated on the left side of these boundaries, oscillation will occur. It is shown that when oscillation occurs, parameter $a$ must be less than the right most value, $a_{\max }$, on the boundary (i.e., at point $b=1$ ). This value can 
be viewed as a necessary condition for oscillation, or bound for failure and repair probabilities, i.e.,

$$
\lambda_{11}+\mu_{11}>1-a_{\max } .
$$

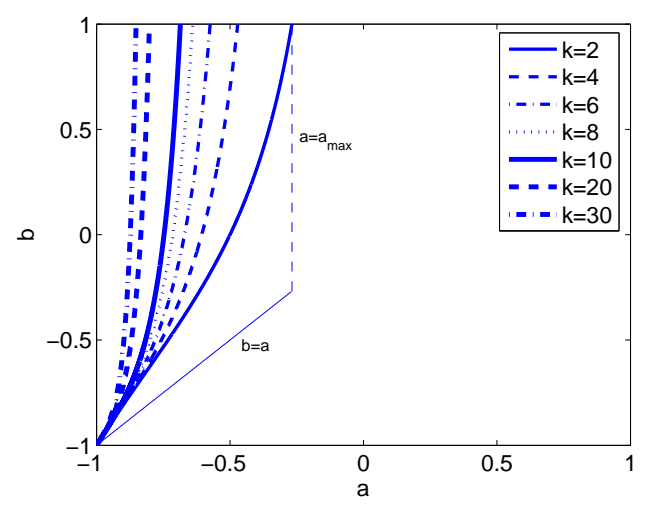

Figure 3.8: Oscillation boundaries with various batch sizes

An illustration of such a bound for $k=2$ is given in Figure 3.8 as the thin broken line. Table 3.3 presents these bounds for different batch size $k$.

Table 3.3: Necessary condition for oscillation

\begin{tabular}{|c|c|c|c|c|}
\hline$k$ & 2 & 4 & 6 & 8 \\
$\lambda_{11}+\mu_{11}>$ & 1.268 & 1.4710 & 1.5744 & 1.6399 \\
\hline$k$ & 10 & 20 & 30 & 50 \\
$\lambda_{11}+\mu_{11}>$ & 1.6859 & 1.8018 & 1.8519 & 1.8920 \\
\hline
\end{tabular}

In addition, Figure 3.8 shows that $a$ is always negative when oscillation occurs. From Equation 3.40 , it is easy to show that in this case $b-a>0$, i.e., $1-\left(\lambda_{21}+\mu_{21}\right)>$ $1-\left(\lambda_{11}+\mu_{11}\right)$. Thus, we obtain another necessary condition or bound for oscillation, as shown in Figure 3.8 with the thin solid line,

$$
\lambda_{21}+\mu_{21}<\lambda_{11}+\mu_{11}
$$

Hence, inequalities 3.41 and 3.42 provide a relative tight bound for oscillation area. Moreover, we observe that the oscillation area is becoming smaller when even batch 
size $k$ is increasing. This implies large batch size reduces the possibility of oscillation.

Clearly, when parameters a and $\mathrm{b}\left(\right.$ or $\left.\lambda_{i 1}, \mu_{i 1}, i=1,2\right)$ are selected that outside of the oscillation area, we always obtain $D_{k+1}<D_{k}$, which implies that the monotonicity properties hold and the product quality will be

- monotonically decreasing with respect to even batch size, i.e., $P_{2 j+1}(g)<P_{2 j}(g), j=$ $1,2, \ldots$, if $e_{11}<e_{21}$, and

- monotonically increasing with respect to even batch size, i.e., $P_{2 j+1}(g)>P_{2 j}(g), j=$ $1,2, \ldots$, if $e_{11}>e_{21}$.

\section{Increasing batch size by 2}

The above results suggest that increasing batch size by 1 may not lead to improvement of product quality. However, when batch size is added by two each time, the monotonic property can always be observed in the equal products case.

Proposition 3.3 Under assumption (1)-(7) in the equal products case, if batch size is added by two, then the product quality is

- monotonically increasing, i.e., $P_{k+2}(g)>P_{k}(g)$, if $e_{11}>e_{21}$;

- monotonically decreasing, i.e., $P_{k+2}(g)<P_{k}(g)$, if $e_{11}<e_{21}$.

Proof: See Appendix A.

Since

$$
P_{k+2}(g)-P_{k}(g)=\frac{\left(\lambda_{21}+\mu_{21}\right)\left(e_{21}-e_{11}\right)}{\lambda_{11}+\mu_{11}}\left(D_{k+2}-D_{k}\right) .
$$




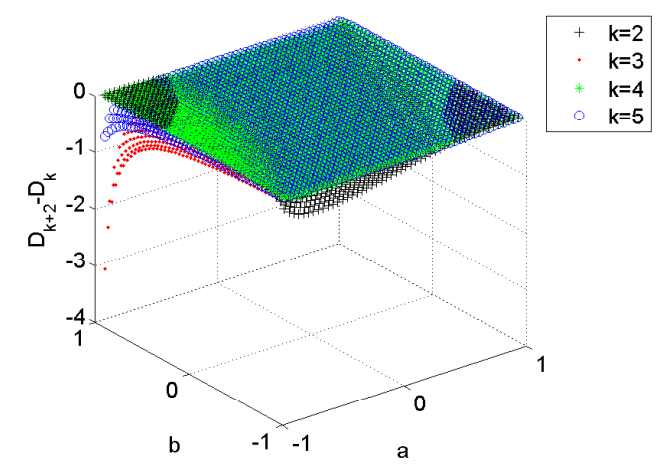

Figure 3.9: $D_{k+2}-D_{k}$ vs $a$ and $b$ with respect to different batch sizes

Proposition 3.3 implies that $D_{k+2}-D_{k}$ is always negative. An illustration of this fact based on numerical investigation is given in Figure 3.9.

Thus, in the equal products case, when quality efficiency without product switch is higher than that with product switch, adding two parts in the batch can improve quality, which agrees with our intuition. Otherwise, quality may be downgraded.

Remark 3.6 In practice, the quality failure probabilities are typically low and the non-monotonic cases are less likely to happen. Effort should be made to avoid occurring of oscillation scenarios so that quality improvement can be achieved.

\subsubsection{Non-monotonic properties in non-equal products case}

For the case of non-equal products, i.e., general case, we investigate the non-monotonic properties using numerical experiments. Specifically, we select quality failure and repair probabilities $\lambda_{i j}, \mu_{i j}$, number of products $n$, and batch size $k_{i}$, randomly and equiprobably from the following sets:

$$
\begin{aligned}
n & \in\{2,3,4,5,6\}, \\
\lambda_{i j} & \in(0,1), \quad i, j=1, \ldots, 6, \\
\mu_{i j} & \in(0,1), \quad i, j=1, \ldots, 6,
\end{aligned}
$$




$$
k_{i} \in\{2,3,4,5,6\}
$$

For a given set of $n, \lambda_{i j}, \mu_{i j}$ and $k_{i}$, quality performance $P(g)$ is calculated and sensitivities to these parameters are investigated. More than 1 million examples are generated for numerical experiments. The percentages that non-monotonic with respect to quality failure/repair probabilities, and batch size are summarized in Tables 3.4 and 3.5 , respectively.

Table 3.4: Percentage of non-monotonic cases with respect to quality failure and repair probabilities (\%)

\begin{tabular}{|c|c|c|c|c|c|}
\hline$n$ & 2 & 3 & 4 & 5 & 6 \\
\hline$\frac{\partial P(g)}{\partial \lambda_{i i}}>0$ & 0.91 & 1.38 & 1.81 & 2.28 & 2.71 \\
\hline$\frac{\partial P(g)}{\partial \mu_{i i}}<0$ & 0.91 & 1.38 & 1.83 & 2.28 & 2.71 \\
\hline$\frac{\partial P(g)}{\partial \lambda_{i j}}>0$ & 1.06 & 1.59 & 2.11 & 2.61 & 3.09 \\
\hline$\frac{\partial P(g)}{\partial \mu_{i j}}<0$ & 1.06 & 1.59 & 2.11 & 2.61 & 3.09 \\
\hline
\end{tabular}

Table 3.5: Percentage of non-monotonic cases with respect to increasing batch size $(\%)$

\begin{tabular}{|c|c|c|c|c|c|}
\hline$n$ & 2 & 3 & 4 & 5 & 6 \\
\hline$k+1$ & 11.52 & 13.04 & 14.19 & 15.12 & 15.89 \\
\hline$k+2$ & 2.12 & 2.87 & 3.27 & 3.63 & 3.91 \\
\hline
\end{tabular}

From these results, it is clear that the non-monotonic properties are observed in all scenarios. We observe that

- In general, the percentages non-monotonic cases occur are typically small.

- The non-monotonic percentage increases with respect to number of products.

- Adding batch size by two leads to significantly smaller percentage of nonmonotonicity compared with adding batch size by one. 
- In addition, the conditions for non-monotonic scenario with respect to quality failure and repair probabilities are similar. In other words, the parameters that results in non-monotonicity to $\lambda_{i i}$ (or $\lambda_{i j}$ ) will most likely lead to nonmonotonicity to $\mu_{i i}$ (respectively, $\mu_{i j}$ ).

- Moreover, by analyzing the quality performance with respect to batch size $k$, we discover that larger batch size leads to smaller percentages of non-monotonic cases.

By carefully analyzing the cases that monotonicity does not hold, we conclude that when these cases are observed, the system parameters typically satisfy at least one or more of the followings:

- Quality efficiency is low, i.e., $e_{i j}<0.5, i, j=1, \ldots, 6$,

- Quality failure probability is high, i.e., $\lambda_{i j}>0.5, i, j=1, \ldots, 6$,

- Quality repair probability is low, i.e., $\mu_{i j}<0.5, i, j=1, \ldots, 6$.

In practice, cases satisfying above conditions seldom happen. This implies that in most practical environment, monotonicity can be expected. However, in the cases of very low quality efficiency, or high failure probability, or low repair probability, more attention is needed in the continuous improvement procedure to avoid any negative impact from possible non-monotonicity.

\subsection{Summary}

In this paper, an analytical method based on Markov chain model is presented to evaluate the quality performance in a flexible manufacturing system with batch pro- 
ductions. Closed formulas to evaluate quality performance are derived and it is also shown that when quality failure probability is relatively high, or repair probability low, or quality efficiency is significantly low, monotonicity may not hold. In addition, more monotonic cases are discovered when increasing buffer size by one compared with increasing by two. Thus, extra care is needed when carrying out continuous improvement project to avoid possible negative effect due to them. In summary, appropriate design of batch policy in flexible manufacturing system is important to maintain good product quality. 


\section{CHAPTER 4}

\section{PRODUCT SEQUENCING WITH RESPECT TO QUALITY}

In this chapter, the impacts of different product sequencing policies on quality are explored. Specially, we investigate which sequence will lead to best quality and which results in worst. In addition, we answer the question whether product quality is improvable or not with respect to resequencing, and we compare quality under different sequencing policies.

\subsection{Two Product Types}

In this simple case, quality formulas in different sequencing polices are derived, and then different polices are compared.

\subsubsection{Quality performance in different sequencing policies}

Consider the simple case of two types of products, 1 and 2, each with batch size two. Since there is only one sequence available, the sequence index $l$ is ignored in this section. From the transition equations (3.5)-3.12), we can find

$$
P\left(g_{11}\right)+P\left(d_{11}\right)=P\left(g_{12}\right)+P\left(d_{12}\right)=P\left(g_{21}\right)+P\left(d_{21}\right)=P\left(g_{22}\right)+P\left(d_{22}\right)=\frac{1}{4} .
$$

By replacing defective states with Equation (4.1), the transition equations can be written as

$$
P\left(g_{11}\right)=\left(1-\lambda_{12}-\mu_{12}\right) P\left(g_{22}\right)+\frac{1}{4} \mu_{12},
$$




$$
\begin{aligned}
& P\left(g_{12}\right)=\left(1-\lambda_{11}-\mu_{11}\right) P\left(g_{11}\right)+\frac{1}{4} \mu_{11}, \\
& P\left(g_{21}\right)=\left(1-\lambda_{21}-\mu_{21}\right) P\left(g_{12}\right)+\frac{1}{4} \mu_{21}, \\
& P\left(g_{22}\right)=\left(1-\lambda_{22}-\mu_{22}\right) P\left(g_{21}\right)+\frac{1}{4} \mu_{22} .
\end{aligned}
$$

Introduce notation

$$
\delta_{i j}=1-\lambda_{i j}-\mu_{i j}, \quad i=1, \ldots, n, \quad j=1, \ldots, k_{i} .
$$

When $\lambda_{i j}+\mu_{i j}=1$, a simplified model is obtained where the quality failure and repair probabilities can be described by Bernoulli trials, thus, we refer it to as Bernoulli-like assumption, i.e.,

$$
\delta_{i j}=0 \text {. }
$$

Although Bernoulli-like assumption is close to the cases in practice, it is still too strict. Thus, this assumption can be relaxed to more general cases, referred to as Bernoulli-relax assumption, i.e.,

$$
0<\left|\delta_{i j}\right|<1, \quad i=1, \ldots, n, \quad j=1, \ldots, k_{i} .
$$

Since in most cases $\delta_{i j}$ is small, we focus on the cases that $\delta_{i j} \ll 1$.

By introducing $\delta_{i j}$, the transition equations can be changed as

$$
\begin{aligned}
& P\left(g_{11}\right)=\delta_{12} P\left(g_{22}\right)+\frac{1}{4} \mu_{12}, \\
& P\left(g_{12}\right)=\delta_{11} P\left(g_{11}\right)+\frac{1}{4} \mu_{11}, \\
& P\left(g_{21}\right)=\delta_{21} P\left(g_{12}\right)+\frac{1}{4} \mu_{21}, \\
& P\left(g_{22}\right)=\delta_{22} P\left(g_{21}\right)+\frac{1}{4} \mu_{22} .
\end{aligned}
$$

Then, we can obtain the overall good part probability as follows: 
Theorem 4.1 Under assumptions (1)-(7) with $n=2$ and $k_{1}=k_{2}=2$, the probability of producing a good part can be calculated by

$$
\begin{aligned}
P\left(g_{b t}\right)= & \frac{1}{4} \cdot \frac{\left(1+\delta_{21}+\delta_{22} \delta_{21}+\delta_{12} \delta_{22} \delta_{21}\right) \mu_{11}}{1-\delta_{11} \delta_{12} \delta_{22} \delta_{21}} \\
& +\frac{1}{4} \cdot \frac{\left(1+\delta_{11}+\delta_{21} \delta_{11}+\delta_{22} \delta_{21} \delta_{11}\right) \mu_{12}}{1-\delta_{11} \delta_{12} \delta_{22} \delta_{21}} \\
& +\frac{1}{4} \cdot \frac{\left(1+\delta_{22}+\delta_{12} \delta_{22}+\delta_{11} \delta_{12} \delta_{22}\right) \mu_{21}}{1-\delta_{11} \delta_{12} \delta_{22} \delta_{21}} \\
& +\frac{1}{4} \cdot \frac{\left(1+\delta_{12}+\delta_{11} \delta_{12}+\delta_{21} \delta_{11} \delta_{12}\right) \mu_{22}}{1-\delta_{11} \delta_{12} \delta_{22} \delta_{21}} .
\end{aligned}
$$

Proof: See Appendix B.

Under the Bernoulli-like assumption, i.e. $\lambda_{i j}+\mu_{i j}=1$ or $\delta_{i j}=0$, the overall good part probability in batch policy can be simplified as

$$
P\left(g_{b t}\right)=\frac{\mu_{11}+\mu_{12}+\mu_{21}+\mu_{22}}{4} .
$$

In strictly sequencing policy, i.e., $k_{i}=1, i=1,2$, the transition equations are

$$
\begin{aligned}
& P\left(g_{11}\right)=\mu_{12} P\left(d_{21}\right)+\left(1-\lambda_{12}\right) P\left(g_{21}\right), \\
& P\left(g_{21}\right)=\mu_{21} P\left(d_{11}\right)+\left(1-\lambda_{21}\right) P\left(g_{11}\right), \\
& P\left(d_{11}\right)=\left(1-\mu_{12}\right) P\left(d_{21}\right)+\lambda_{12} P\left(g_{21}\right), \\
& P\left(d_{21}\right)=\left(1-\mu_{21}\right) P\left(d_{11}\right)+\lambda_{21} P\left(g_{11}\right) .
\end{aligned}
$$

and the additional constraint is

$$
P\left(g_{11}\right)+P\left(d_{11}\right)+P\left(g_{21}\right)+P\left(d_{21}\right)=1 .
$$

Furthermore, the transition equations related to $\delta_{i j}$ are written as

$$
\begin{aligned}
& P\left(g_{11}\right)=\delta_{12} P\left(g_{21}\right)+\frac{1}{2} \mu_{12}, \\
& P\left(g_{21}\right)=\delta_{21} P\left(g_{11}\right)+\frac{1}{2} \mu_{21} .
\end{aligned}
$$


Then, we can obtain the overall good part probability as follows:

Theorem 4.2 Under assumptions (1)-(7) with $n=2$, the probability of producing a good part can be calculated by

$$
P\left(g_{s s}\right)=\frac{1}{2} \cdot \frac{\left(1+\delta_{12}\right) \mu_{21}+\left(1+\delta_{21}\right) \mu_{12}}{1-\delta_{21} \delta_{12}}
$$

Under the Bernoulli-like assumption, the overall good part probability in strictly sequencing policy can be simplified as

$$
P\left(g_{s s}\right)=\frac{\mu_{12}+\mu_{21}}{2}
$$

\subsubsection{Sequencing comparison under Bernoulli-like assumption}

Quality performance in batch policy (Equations (4.10) ) consists of the sum of all quality repair probabilities within a batch and between batches; while quality performance in strictly sequencing policy (Equations (4.12)) depends on quality repair probabilities within a batch and between batches only. Therefore, for two types of product, we define the total quality repair probability and total quality efficiency without product switch as

$$
\begin{aligned}
& \mu_{\text {no-switch }}^{\text {total }}=\mu_{11}+\mu_{22}, \\
& e_{\text {no-switch }}^{\text {total }}=e_{11}+e_{22},
\end{aligned}
$$

and the total quality repair probability and total quality efficiency with product switch as

$$
\begin{aligned}
& \mu_{\text {switch }}^{\text {total }}=\mu_{12}+\mu_{21}, \\
& e_{\text {switch }}^{\text {total }}=e_{12}+e_{21} .
\end{aligned}
$$


Under the Bernoulli-like assumption, it can be easily obtained

$$
\begin{aligned}
\mu_{\text {no-switch }}^{\text {total }} & =e_{\text {no-switch }}^{\text {total }} \\
\mu_{\text {switch }}^{\text {total }} & =e_{\text {switch }}^{\text {total }}
\end{aligned}
$$

Proposition 4.1 Under assumptions (1)-(7) and Bernoulli-like assumption (4.3), for $n=2$, the following statements hold:

$$
\begin{aligned}
& \text { (1) } P\left(g_{b t}\right)>P\left(g_{s s}\right) \text { if } \mu_{\text {no-switch }}^{\text {total }}>\mu_{\text {switch }}^{\text {total }}, \\
& \text { (2) } P\left(g_{b t}\right)<P\left(g_{s s}\right) \text { if } \mu_{\text {no-switch }}^{\text {total }}<\mu_{\text {switch }}^{\text {total }}
\end{aligned}
$$

This corollary indicates that when the total quality efficiency without product switch is higher, using batch policy can achieve better quality performance compared with strictly sequencing policy.

\subsubsection{Sequencing comparison under Bernoulli-relax assumption}

Define $\delta_{\text {max }}=\max _{i j}\left|\delta_{i j}\right|, i, j=1,2$. Then, we obtain the following results:

Proposition 4.2 Under assumptions (1)-(7) and Bernoulli-relax assumption (4.4), for $n=2$, the following statements hold:

$$
\begin{aligned}
& \text { (1) } \quad P\left(g_{b t}\right)>P\left(g_{s s}\right) \text { if } \mu_{n o-s w i t c h}^{\text {total }}>\mu_{\text {switch }}^{\text {total }} \text { and } \\
& 0<\delta_{\text {max }}<\frac{\mu_{\text {no-switch }}^{\text {total }}-\mu_{\text {switch }}^{\text {total }}}{\mu_{\text {no-switch }}^{\text {total }}+3 \mu_{\text {switch }}^{\text {total }}}, \\
& P\left(g_{b t}\right)<P\left(g_{s s}\right) \quad \text { if } \mu_{\text {no-switch }}^{\text {total }}<\mu_{\text {switch }}^{\text {total }} \text { and } \\
& 0<\delta_{\text {max }}<\frac{\mu_{\text {switch }}^{\text {total }}-\mu_{\text {no-switch }}^{\text {total }}}{\mu_{\text {no-switch }}^{\text {total }}+3 \mu_{\text {switch }}^{\text {total }}} .
\end{aligned}
$$

Proof: See Appendix B. 


\subsection{General Multiple Product Types}

In general case, quality formulas in different sequencing polices are derived, and then different polices are compared.

\subsubsection{Quality performance in different sequencing policies}

Consider the case of $n$ types of product, denoted as $1,2, \ldots, n$. Then, there are $(n-1)$ ! permutations of sequence. For an arbitrary sequence $s^{l}=\left\{s_{1}^{l}, s_{2}^{l}, \cdots, s_{n}^{l}\right\}$, we obtain the transition equations in steady states

$$
\begin{gathered}
P\left(g_{s_{i}^{l}, j}\right)=\mu_{s_{i}^{l}, s_{i}^{l}} P\left(d_{s_{i}^{l}, j-1}\right)+\left(1-\lambda_{s_{i}^{l}, s_{i}^{l}}\right) P\left(g_{s_{i}^{l}, j-1}\right), \\
P\left(d_{s_{i}^{l}, j}\right)=\left(1-\mu_{s_{i}^{l}, s_{i}^{l}}\right) P\left(d_{s_{i}^{l}, j-1}\right)+\lambda_{s_{i}^{l}, s_{i}^{l}} P\left(g_{s_{i}^{l}, j-1}\right), \\
i=1, \ldots, n, j=2, \ldots, k_{s_{i}^{l}}, \\
P\left(g_{s_{i}^{l}, 1}\right)=\mu_{s_{i}^{l}, s_{i-1}^{l}} P\left(d_{s_{i-1}^{l}, k_{s_{i-1}^{l}}}\right)+\left(1-\lambda_{s_{i}^{l}, s_{i-1}^{l}}\right) P\left(g_{s_{i-1}^{l}, k_{s_{i-1}^{l}}}\right), \\
P\left(d_{s_{i}^{l}, 1}\right)=\left(1-\mu_{s_{i}^{l}, s_{i-1}^{l}}\right) P\left(d_{s_{i-1}^{l}, k_{s_{i-1}^{l}}}\right)+\lambda_{s_{i}^{l}, s_{i-1}^{l}} P\left(g_{s_{i-1}^{l}, k_{s_{i-1}^{l}}}\right), \\
i=2, \ldots, n, \\
P\left(g_{s_{1}^{l}, 1}\right)=\mu_{s_{1}^{l}, s_{n}^{l}} P\left(d_{s_{n}^{l}, k_{s_{n}^{l}}}\right)+\left(1-\lambda_{s_{1}^{l}, s_{n}^{l}}\right) P\left(g_{s_{n}^{l}, k_{s_{n}^{l}}}\right), \\
P\left(d_{s_{1}^{l}, 1}\right)=\left(1-\mu_{s_{1}^{l}, s_{n}^{l}}\right) P\left(d_{s_{n}^{l}, k_{s_{n}^{l}}}\right)+\lambda_{s_{1}^{l}, s_{n}^{l}} P\left(g_{s_{n}^{l}, k_{s_{n}^{l}}}\right) .
\end{gathered}
$$

In addition, the total probability is equal to 1 ,

$$
\sum_{s_{i}^{l}=1}^{n} \sum_{j=1}^{k_{s_{i}^{l}}}\left[P\left(g_{s_{i}^{l}, j}\right)+P\left(d_{s_{i}^{l}, j}\right)\right]=1 .
$$

These expressions can be further reduced by summing up $P\left(g_{s_{i}^{l}, j}\right)$ and $P\left(d_{s_{i}^{l}, j}\right)$. Then we obtain

$$
P\left(g_{s_{i}^{l}, j}\right)+P\left(d_{s_{i}^{l}, j}\right)=P\left(g_{s_{i}^{l}, 1}\right)+P\left(d_{s_{i}^{l}, 1}\right)=P\left(g_{s_{1}^{l}, 1}\right)+P\left(d_{s_{1}^{l}, 1}\right)
$$


which leads to

$$
P\left(g_{s_{i}^{l}, j}\right)+P\left(d_{s_{i}^{l}, j}\right)=\frac{1}{K}, \quad i=1, \ldots, n, \quad j=1, \ldots, k_{s_{i}^{l}} .
$$

Recall the notation

$$
\delta_{s_{i}^{l}, s_{j}^{l}}=1-\lambda_{s_{i}^{l}, s_{j}^{l}}-\mu_{s_{i}^{l}, s_{j}^{l}}, \quad i, j=1, \ldots, n .
$$

Then the transition equations can be rewritten as

$$
\begin{aligned}
& P\left(g_{s_{i}^{l}, j}\right)=\delta_{s_{i}^{l}, s_{i}^{l}} P\left(g_{s_{i}^{l}, j-1}\right)+\frac{\mu_{s_{i}^{l}, s_{i}^{l}}}{K}, \quad i=1, \ldots, n, j=2, \ldots, k_{s_{i}^{l}}, \\
& P\left(g_{s_{i}^{l}, 1}\right)=\delta_{s_{i}^{l}, s_{i-1}^{l}} P\left(g_{s_{i-1}^{l}, k_{s_{i-1}^{l}}}\right)+\frac{\mu_{s_{i}^{l}, s_{i-1}^{l}}}{K}, \quad i=2, \ldots, n, \\
& P\left(g_{s_{1}^{l}, 1}\right)=\delta_{s_{1}^{l}, s_{n}^{l}} P\left(g_{s_{n}^{l}, k_{s_{n}^{l}}}\right)+\frac{\mu_{s_{1}^{l}, s_{n}^{l}}}{K} .
\end{aligned}
$$

Introduce vectors $X, \Phi$ and matrix $\Gamma$ such that

$$
\begin{aligned}
X & =\left[P\left(g_{s_{1}^{l}, 1}\right), \ldots, P\left(g_{s_{1}^{l}, k_{s_{1}^{l}}}\right), P\left(g_{s_{2}^{l}, 1}\right), \ldots, P\left(g_{s_{2}^{l}, k_{s_{2}^{l}}}\right), \ldots, P\left(g_{s_{n}^{l}, 1}\right), \ldots, P\left(g_{s_{n}^{l}, k_{s_{n}^{l}}}\right)\right]^{T}, \\
\Phi= & {\left[\mu_{s_{1}^{l}, s_{n}^{l}}, \mu_{s_{1}^{l}, s_{1}^{l}}, \ldots, \mu_{s_{1}^{l}, s_{1}^{l}}, \mu_{s_{2}^{l}, s_{1}^{l}}, \mu_{s_{2}^{l}, s_{2}^{l}}, \ldots, \mu_{s_{n}^{l}, s_{n-1}^{l},}, \mu_{s_{n}^{l}, s_{n}^{l}}, \ldots, \mu_{s_{n}^{l}, s_{n}^{l}}\right]^{T}, }
\end{aligned}
$$


Then the transition equations can be written in matrix form

$$
X=\Gamma X+\frac{1}{K} \Phi
$$

It follows that

$$
X=\frac{1}{K}(I-\Gamma)^{-1} \Phi
$$

Note that the inverse always exists due to the fact that an irreducible Markov chain with finite number of states has a unique solution. Moreover, we can further rewrite $X$ into the following (See Appendix B for detailed derivation):

$$
X=\frac{1}{K} \cdot \frac{\sum_{i=1}^{K} \Gamma^{i-1}}{\operatorname{det}(I-\Gamma)} \Phi .
$$

Therefore, the probability of good parts $P\left(g_{b t}^{l}\right)$ is evaluated as follows:

Theorem 4.3 Under assumptions (1)-(7), the probability of good parts $P\left(g_{b t}^{l}\right)$ in batch policy is calculated by

$$
P\left(g_{b t}^{l}\right)=\sum_{i=1}^{K} x_{i}
$$

where $K=\sum_{i=1}^{n} k_{s_{i}^{l}}$, and $x_{i}$ is element of $X$ and can be solved from (4.34).

Remark 4.1 Consider the strictly sequencing policy, i.e., product type changes every cycle. In other words, batch size $k_{i}=1, \forall i$. Then,

$$
\begin{aligned}
X= & {\left[P\left(g_{s_{1}^{l}, 1}\right), P\left(g_{s_{2}^{l}, 1}\right), \ldots, P\left(g_{s_{n}^{l}, 1}\right)\right]^{T}, } \\
\Phi= & {\left[\mu_{s_{1}^{l}, s_{n}^{l}}, \mu_{s_{2}^{l}, s_{1}^{l}}, \ldots, \mu_{s_{n}^{l}, s_{n-1}^{l}}\right]^{T}, } \\
\Gamma= & \left(\begin{array}{cccc}
0 & \cdots & 0 & \delta_{s_{1}^{l}, s_{n}^{l}} \\
\delta_{s_{2}^{l}, s_{1}^{l}} & \cdots & 0 & 0 \\
0 & \ddots & 0 & 0 \\
0 & \cdots & \delta_{s_{n}^{l}, s_{n-1}^{l}} & 0
\end{array}\right) .
\end{aligned}
$$


The product quality, denoted as $P\left(g_{s s}^{l}\right)$, under this policy can also be calculated as

$$
P\left(g_{s s}^{l}\right)=\sum_{i=1}^{n} x_{i},
$$

where again $x_{i}$ is calculated from (4.34).

Remark 4.2 In many manufacturing plants, $100 \%$ inspection is carried out after critical operations. For example, "finess" (inspection) typically is designed after painting booths and ovens, where every vehicle will be inspected for paint quality. In such systems, we can view the vehicle having "good" quality if it passes inspection, and "defective" if paint defect is discovered. With a relative long time period, based on the data $\log$ of each shift collected on the factory floor, the transitions among vehicles with different colors can be obtained, and probabilities $\lambda_{i j}$ and $\mu_{i j}$ can be calculated. For systems without 100\% inspection, a larger amount of inspection data is needed in order to obtain all transition probabilities. Then, using these probabilities, Theorem 4.3 can be used to evaluate the product quality (in terms of good part ratio).

Assume $\delta_{s_{i}^{l}, s_{j}^{l}}=0, \forall i, j$, i.e.,

$$
\lambda_{s_{i}^{l}, s_{j}^{l}}+\mu_{s_{i}^{l}, s_{j}^{l}}=1, \quad i, j=1, \ldots, n .
$$

We obtain a simplified model where the quality failure and repair probabilities can be described by Bernoulli trials, thus, we refer it to as Bernoulli-like assumption. Then, the probability to produce a good part for a given sequence $s^{l}$ in batch production can be simplified as: 
Corollary 4.1 Under assumptions (1)-(7) and Bernoulli assumption (4.41), the probability of good parts $P\left(g_{b t}^{l}\right)$ in batch policy is calculated by

$$
P\left(g_{b t}^{l}\right)=\frac{\sum_{i=1}^{n}\left(k_{s_{i}^{l}}-1\right) \mu_{s_{i}^{l}, s_{i}^{l}}+\sum_{i=2}^{n} \mu_{s_{i}^{l}, s_{i-1}^{l}}+\mu_{s_{1}^{l}, s_{n}^{l}}}{\sum_{i=1}^{n} k_{s_{i}^{l}}} .
$$

Proof: Immediately follows by $\Gamma=0$ in equation 4.34.

Remark 4.3 In case of strictly sequencing policy, $P\left(g_{s s}^{l}\right)$ is easy to obtain as:

$$
P\left(g_{s s}^{l}\right)=\frac{\sum_{i=2}^{n} \mu_{s_{i}^{l}, s_{i-1}^{l}}+\mu_{s_{1}^{l}, s_{n}^{l}}}{n} .
$$

Remark 4.4 In practice, the quality failure probability $\lambda_{i j}$ is typically small, and quality repair probability $\mu_{i j}$ is usually large, close to 1 . Thus, the sum of $\lambda_{i j}$ and $\mu_{i j}$ is close to 1 . Therefore, the Bernoulli-like assumption is close to the scenario in real world.

\subsubsection{A paint shop example}

As indicated in Chapter 1, appropriate sequencing of different types of products could improve the overall quality. In this subsection, a paint shop example is studied under the Bernoulli-like assumption to obtain some intuition. Consider an automotive paint shop and assume that there are three color options for vehicles, white, grey and black. Clearly, there are two possible sequence options available,

$$
\begin{aligned}
& s^{1}=\{\text { white, black, grey }\} \\
& s^{2}=\{\text { white, grey, black }\} .
\end{aligned}
$$

\begin{tabular}{|c|c|c|c|c|}
\hline$\lambda_{\text {white,black }}$ & $\lambda_{\text {white,grey }}$ & $\lambda_{\text {black,white }}$ & $\lambda_{\text {black,grey }}$ & $\lambda_{\text {grey,white }}$ \\
\hline$u_{\text {white,black }}$, & $\mu_{w h i t e, g r e y}$, & $\mu_{\text {black,white }}$, & $\mu_{\text {black,grey }}$, & $\mu_{\text {grey,white }}$ \\
\hline
\end{tabular}

The product quality will be dependent on the transition probabilities: 
When these probabilities are known, using Theorem 4.3 , the product quality can be calculated for both sequences.

Although the product sequence can be selected by searching the whole space of possible combinations, in order to understand the nature of the system, we would like to investigate the relationship between product quality and sequence options. For example, which policy, strictly sequencing or batch, will result in better quality? Within each policy, which sequence will lead to better quality? Answers to such questions could provide more guidance for designing product sequence to ensure desired quality in both design and operation phases. In addition, it is natural to question whether both policies can adopt the same sequences to improve quality. In other words, if sequence $s^{1}$ has better quality in one policy comparing with sequence $s^{2}$, will this property hold in another policy? Moreover, some transitions have lower quality efficiencies than other transitions, will the sequence with the worst transition with respect to quality efficiency be necessarily the worst sequence in terms of quality? Or will the sequence with the best transition be necessarily the best sequence? Providing answers to these questions is of importance in practice.

From equations 4.42 and (4.43), we obtain

$$
\begin{aligned}
P\left(g_{s s}^{1}\right)= & \frac{\mu_{\text {white, } \text { grey }}+\mu_{\text {grey,black }}+\mu_{\text {black }, \text { white }}}{3}, \\
P\left(g_{s s}^{2}\right)= & \frac{\mu_{\text {white }, \text { black }}+\mu_{\text {black,grey }}+\mu_{\text {grey }, \text { white }}}{3}, \\
P\left(g_{b t}^{1}\right)= & \frac{\mu_{\text {white, grey }}+\mu_{\text {grey,black }}+\mu_{\text {black }, \text { white }}}{k_{\text {white }}+k_{\text {black }}+k_{\text {grey }}} \\
& +\frac{\left(k_{\text {white }}-1\right) \mu_{\text {white }, \text { white }}+\left(k_{\text {black }}-1\right) \mu_{\text {black,black }}+\left(k_{\text {grey }}-1\right) \mu_{\text {grey }, \text { grey }}}{k_{\text {white }}+k_{\text {black }}+k_{\text {grey }}}, \\
P\left(g_{b t}^{2}\right)= & \frac{\mu_{\text {white,black }}+\mu_{\text {black,grey }}+\mu_{\text {grey }, \text { white }}}{k_{\text {white }}+k_{\text {black }}+k_{\text {grey }}}
\end{aligned}
$$




$$
+\frac{\left(k_{\text {white }}-1\right) \mu_{\text {white, white }}+\left(k_{\text {black }}-1\right) \mu_{\text {black black }}+\left(k_{\text {grey }}-1\right) \mu_{\text {grey,grey }}}{k_{\text {white }}+k_{\text {black }}+k_{\text {grey }}} .
$$

From the above, the following properties are observed:

- An individual best or worst transition could not quantify whether a sequence results in better quality or not. The summation of all transition probabilities with product changes in a sequence determines the quality of the sequence.

- Since the second fraction terms in batch policy are same for both sequences, identical comparison results will be obtained in both strictly sequencing and batch policies. In other words, if one sequence is better under strictly sequencing policy, it again has better quality under batch policy, and vice versa.

- Due to the same rationale, only the transitions between different product types play an role in comparing different sequences.

- Again, due to the same inference, these results are independent of batch size $k_{i}$.

Intuitively we understand that it is much easier to maintain good paint quality to paint a black vehicle after a white one, comparing with reversed sequence. This is typical in many paint shops that the transition quality from white to black is much better than that from black to white, and the difference between white to grey and grey to white is smaller, and also smaller is the difference between grey to black and black to grey. Therefore, sequence $s^{1}$ typically results in better quality than $s^{2}$. This explains why black vehicle is usually arranged after white and not vice versa in practical operations. 
Such results provide some hints for more general cases with $n$ product types and non-identical batch sizes. Below, analysis will be carried out to extend them.

\subsubsection{Product sequencing under Bernoulli-like assumption Optimal sequences under strictly sequencing and batch policies}

Recall the definition of quality efficiency

$$
e_{s_{i}^{l}, s_{j}^{l}}=\frac{\mu_{s_{i}^{l}, s_{j}^{l}}}{\lambda_{s_{i}^{l}, s_{j}^{l}}+\mu_{s_{i}^{l}, s_{j}^{l}}}, \quad i, j=1, \ldots, n .
$$

As one can see, under Bernoulli-like assumption, we have $e_{s_{i}^{l}, s_{j}^{l}}=\mu_{s_{i}^{l}, s_{j}^{l}}, \forall i, j$. Thus, from 4.43 we obtain

Proposition 4.3 Under assumptions (1)-(7) and Bernoulli-like assumption (4.41), the optimal sequence in strictly sequencing policy is the one that satisfies

$$
\max _{s^{l}}\left\{\sum_{i=2}^{n} e_{s_{i}^{l}, s_{i-1}^{l}}+e_{s_{1}^{l}, s_{n}^{l}}\right\} .
$$

It is easy to see from 4.42 that the transition probabilities within batches do not play a role in searching for optimal ones. Hence, such sequence will keep optimality under batch policy, independent of the batch size $k_{i}, \forall i$. Therefore,

Proposition 4.4 Under assumptions (1)-(7) and Bernoulli-like assumption (4.41), the optimal sequence in batch policy is the one that satisfies

$$
\max _{s^{l}}\left\{\sum_{i=2}^{n} e_{s_{i}^{l}, s_{i-1}^{l}}+e_{s_{1}^{l}, s_{n}^{l}}\right\} .
$$

Remark 4.5 It is clear that the worst sequence for both policies would be

$$
\min _{s^{l}}\left\{\sum_{i=2}^{n} e_{s_{i}^{l}, s_{i-1}^{l}}+e_{s_{1}^{l}, s_{n}^{l}}\right\} .
$$

Such a sequence is referred to as a Bottleneck Sequence (BN-s), which should be avoided in operations. 


\section{Sequence comparison under strictly sequencing and batch policies}

Propositions 4.3 and 4.4 indicate that the optimal sequence is only dependent on the transitions between different product types. Thus, an optimal sequence in one policy will still be optimal in another one. It is natural to question that if comparing to another sequence, one sequence leads to better quality in one policy, such property will still hold or not in another policy. The answer is given below:

Proposition 4.5 Under assumptions (1)-(7) and Bernoulli-like assumption (4.41), the following statement holds for any sequences $s^{l}$ and $s^{m}, l, m \in\{1,2, \ldots,(n-1) !\}$ :

$$
P\left(g_{b t}^{l}\right)>P\left(g_{b t}^{m}\right) \quad \text { if and only if } \quad P\left(g_{s s}^{l}\right)>P\left(g_{s s}^{m}\right), \quad l, m \in\{1,2, \ldots,(n-1) !\} .
$$

Proof: See Appendix B.

Therefore, we only need to compare different sequences under one policy, and the results will be applicable under the other policy.

\section{Comparison between strictly sequencing and batch policies}

First assume all $k_{i}=k, \forall i$. For a given sequence $s^{l}$, equation 4.42 can be simplified as

$$
P\left(g_{b t}^{l}\right)=\frac{(k-1) \sum_{i=1}^{n} \mu_{s_{i}^{l}, s_{i}^{l}}+\sum_{i=2}^{n} \mu_{s_{i}^{l}, s_{i-1}^{l}}+\mu_{s_{1}^{l}, s_{n}^{l}}}{n k} .
$$

Then, comparing with the same sequence under strictly sequencing policy, we have

$$
\begin{aligned}
P\left(g_{b t}^{l}\right)-P\left(g_{s s}^{l}\right) & =\frac{(k-1) \sum_{i=1}^{n} \mu_{s_{i}^{l}, s_{i}^{l}}+\sum_{i=2}^{n} \mu_{s_{i}^{l}, s_{i-1}^{l}}+\mu_{s_{1}^{l}, s_{n}^{l}}}{n k}-\frac{\sum_{i=2}^{n} \mu_{s_{i}^{l}, s_{i-1}^{l}}+\mu_{s_{1}^{l}, s_{n}^{l}}}{n} \\
& =\frac{k-1}{n k} \cdot\left[\sum_{i=1}^{n} \mu_{s_{i}^{l}, s_{i}^{l}}-\left(\sum_{i=2}^{n} \mu_{s_{i}^{l}, s_{i-1}^{l}}+\mu_{s_{1}^{l}, s_{n}^{l}}\right)\right] .
\end{aligned}
$$


Clearly, the first term in the bracket is the sum of all quality repair probabilities within a batch, while the second term is the sum of all quality repair probabilities between batches. Therefore, we define the total quality repair probability and total quality efficiency without product switch as

$$
\mu_{n o-s w i t c h}^{\text {total }}=\sum_{i=1}^{n} \mu_{s_{i}^{l}, s_{i}^{l}}=e_{n o-s w i t c h}^{\text {total }}=\sum_{i=1}^{n} e_{s_{i}^{l}, s_{i}^{l}}
$$

and the total quality repair probability and total quality efficiency with product switch as

$$
\mu_{\text {switch }}^{\text {total }}=\sum_{i=2}^{n} \mu_{s_{i}^{l}, s_{i-1}^{l}}+\mu_{s_{1}^{l}, s_{n}^{l}}=e_{\text {switch }}^{\text {total }}=\sum_{i=2}^{n} e_{s_{i}^{l}, s_{i-1}^{l}}+e_{s_{1}^{l}, s_{n}^{l}} .
$$

The difference between batch and strictly sequencing policies in product quality is determined by

$$
\mu_{\text {no-switch }}^{\text {total }}-\mu_{\text {switch }}^{\text {total }}=e_{\text {no-switch }}^{\text {total }}-e_{\text {switch }}^{\text {total }} .
$$

Proposition 4.6 Under assumptions (1)-(7) and Bernoulli-like assumption (4.41) for all $k_{i}=k, \forall i$, the following statement holds:

$$
P\left(g_{b t}^{l}\right)>P\left(g_{s s}^{l}\right) \quad \text { if and only if } e_{\text {no-switch }}^{\text {total }}>e_{\text {switch }}^{\text {total }}
$$

When batch size $k_{i}$ is not same for all products under batch policy, the product composition will not be the same as that under strictly sequencing policy (which is $1 / n$ for all products). Thus, direct comparison between two policies is not reasonable. Therefore, the weight information of different products should be included in the comparison. To compensate this, we propose a modified comparison based on the weight of different products. Define

$$
P(i)=\frac{k_{i}}{\sum_{i=1}^{n} k_{i}}
$$


as the percentage of product type $i$ among all products. Then modify $e_{n o-s w i t c h}^{\text {total }}$ and $e_{\text {switch }}^{\text {total }}$ to

$$
\begin{aligned}
\widetilde{e}_{\text {no-switch }}^{\text {total }} & =\sum_{i=1}^{n}\left[P\left(s_{i}^{l}\right)-\frac{1}{\sum_{i=1}^{n} k_{s_{i}^{l}}}\right] e_{s_{i}^{l}, s_{i}^{l}}, \\
\widetilde{e}_{\text {switch }}^{\text {total }} & =\left(\frac{1}{n}-\frac{1}{\sum_{i=1}^{n} k_{s_{i}^{l}}}\right)\left(e_{s_{1}^{l}, s_{n}^{l}}+\sum_{i=2}^{n} e_{s_{i}^{l}, s_{i-1}^{l}}\right) .
\end{aligned}
$$

It is clear that the relationship between $\widetilde{e}_{n o-\text { switch }}^{\text {total }}$ and $\widetilde{e}_{\text {switch }}^{\text {total }}$ will be the same as that between $e_{n o-s w i t c h}^{\text {total }}$ and $e_{\text {switch }}^{\text {total }}$ if all $k_{i}$ s are identical. It can be shown that, after this modification, the previous property still holds even in the case of $k_{i} \neq k_{j}, i \neq j$, $i, j=1, \ldots, n$.

Proposition 4.7 Under assumptions (1)-(7) and Bernoulli assumption (4.41) with any batch size $k_{i}, \forall i$, the following statement holds:

$$
P\left(g_{b t}^{l}\right)>P\left(g_{s s}^{l}\right) \quad \text { if and only if } \widetilde{e}_{n o-s w i t c h}^{t o t a l}>\widetilde{e}_{\text {switch }}^{\text {total }}
$$

Proof: See Appendix B.

Therefore, as long as the total quality efficiency within the batch is better than that between batches, implementing batch policy is always superior in quality than using strictly sequencing policy.

Remark 4.6 In our experiences, when the defects are introduced due to change of products, such as location errors in machining operations, incomplete purging in painting operations, mis-matching parts in assembly operations, etc., the quality efficiencies are typically better without product switch. In this case, batch operation is preferred. However, if the defects are caused by machines or loaded materials, such 
as bad mixer of paints, wrongly labeled carts of parts, etc., batch production may be detrimental since defective parts are produced continuously. In this case, quality efficiency within the batch is typically small. Therefore, condition 4.52 provides a quantitative measure for policy selection.

\subsubsection{Product sequencing under Bernoulli-relax assumption}

Although Bernoulli-like assumption is close to the cases in practice, it is still too strict. Thus, we relax this assumption to more general cases, referred to as the Bernoulli-relax assumption, i.e.,

$$
0<\left|\delta_{s_{i}^{l}, s_{j}^{l}}\right|<1, \quad i, j=1, \ldots, n
$$

Since in most cases $\delta_{s_{i}^{l}, s_{j}^{l}}$ is small, we focus on the cases that $\delta_{s_{i}^{l}, s_{j}^{l}} \ll 1$.

\section{Optimal sequences under strictly sequencing and batch policies}

Recall that under Bernoulli-like assumption we conclude that the optimal sequence to deliver best quality is the sequence with the largest $\sum_{i=2}^{n} \mu_{s_{i}^{l}, s_{i-1}^{l}}+\mu_{s_{1}^{l}, s_{n}^{l}}$ or $\sum_{i=2}^{n} e_{s_{i}^{l}, s_{i-1}^{l}}+$ $e_{s_{1}^{l}, s_{n}^{l}}$. Such result may not be true when Bernoulli-like assumption is relaxed, since now $\mu_{i j}$ is not equal to $e_{i j}$. However, the following relationship exists:

Proposition 4.8 Under assumptions (1)-(7) and Bernoulli-relax assumption (4.53), for a given sequence $s^{l}$, the following statement on product quality $P\left(g^{l}\right)$ is true:

$$
\frac{1}{1+\delta_{\max }} \widetilde{\mu}^{l} \leq P\left(g^{l}\right) \leq \frac{1}{1-\delta_{\max }} \widetilde{\mu}^{l}
$$

where $\delta_{\max }=\max _{i j}\left|\delta_{s_{i}^{l}, s_{j}^{l}}\right|, \forall i, j$, and

$$
\widetilde{\mu}^{l}=\frac{\sum_{i=2}^{n} \mu_{s_{i}^{l}, s_{i-1}^{l}}+\mu_{s_{1}^{l}, s_{n}^{l}}}{n}:=\widetilde{\mu}_{s s}^{l}
$$


for strictly sequencing policy and

$$
\widetilde{\mu}^{l}=\frac{\sum_{i=1}^{n}\left(k_{s_{i}^{l}}-1\right) \mu_{s_{i}^{l}, s_{i}^{l}}+\sum_{i=2}^{n} \mu_{s_{i}^{l}, s_{i-1}^{l}}+\mu_{s_{1}^{l}, s_{n}^{l}}}{\sum_{i=1}^{n} k_{s_{i}^{l}}}:=\widetilde{\mu}_{b t}^{l}
$$

for batch policy.

Proof: See Appendix B.

It is easy to see that $\widetilde{\mu}^{l}$ represents the product quality in Bernoulli-like case with the same $\mu_{i j}^{l}$. From 4.54, it can be further deduced that

$$
\frac{\delta_{\max }}{1+\delta_{\max }} \leq\left|\frac{P\left(g^{l}\right)-\widetilde{\mu}^{l}}{P\left(g^{l}\right)}\right| \leq \frac{\delta_{\max }}{1-\delta_{\max }} .
$$

When $\delta_{\max } \ll 1$, we obtain that the difference between $P\left(g^{l}\right)$ and $\widetilde{\mu}^{l}$ will be close to $\delta_{\max }$, which is small. Therefore, under this condition, we may apply the criterion in Bernoulli-like case to determine the optimal sequence.

To investigate the effectiveness of this condition, i.e.,

$$
\max _{s^{l}}\left\{\sum_{i=2}^{n} e_{s_{i}^{l}, s_{i-1}^{l}}+e_{s_{1}^{l}, s_{n}^{l}}\right\}
$$

to test optimality under Bernoulli-relax assumption, we carry out numerical experiments by randomly chosen $\delta_{i j}$. Specifically, for a given $\delta_{\text {max }}$, we randomly and equiprobably select $\mu_{i j}$ and $\lambda_{i j}$ such that

$$
\begin{aligned}
& \lambda_{i j} \in\left(0, \delta_{\max }\right], \\
& \mu_{i j} \in\left[1-\delta_{\max }, 1\right),
\end{aligned}
$$

which implies that

$$
1-\delta_{\max }<\lambda_{i j}+\mu_{i j}<1+\delta_{\max }
$$


The experiment is repeated 10,000 times for $n=3$ to 6 with different $\delta_{\max }$, respectively. The optimal sequences selected by complete search of the possible combinations are compared with the sequences chosen using condition (4.57). When both the chosen sequences are identical, condition (4.57) results in an effective selection. The percentages of correct selections under different scenarios are summarized in Table 4.1 for $\delta_{\max }=0.1$ and 0.2 . It is clear that when $\delta_{\max }$ is small, which is the scenario of majority cases, condition (4.57) results in a high percentage of correctness, typically more than $90 \%$.

Table 4.1: Correctness of condition 4.57) (\%)

\begin{tabular}{|c|c|c|c|c|}
\hline$n$ & 3 & 4 & 5 & 6 \\
\hline$\delta_{\max }=0.1$ & 98.22 & 96.25 & 94.71 & 93.20 \\
\hline$\delta_{\max }=0.2$ & 96.51 & 92.70 & 89.65 & 87.53 \\
\hline
\end{tabular}

Even if condition (4.57) does not select the optimal sequence, a sub-optimal one is typically chosen. In Table 4.2, the cases of incorrect selections are analyzed. First, the percentage that condition 4.57 selects the sequence with the second largest quality is presented. It can be seen that among all the incorrectly chosen cases, roughly $90 \%$ and $80 \%$ of them result in selecting the sequence with the second large quality based on condition (4.57), for $\delta_{\max }=0.1$ and 0.2 , respectively. Moreover, we investigate the differences in quality between the optimal sequence and the one selected using condition (4.57). As one can see that such differences are extremely small.

Remark 4.7 The above numerical experiments are carried out when $\delta_{\max }$ is small, which is the majority of cases in practice. For completeness of the study, we also investigate the cases when $\delta_{\max }$ is large. As we expect, the percentage of 
Table 4.2: Errors when condition 4.57) has incorrect selections

\begin{tabular}{|c|c|c|c|c|}
\hline$n$ & 3 & 4 & 5 & 6 \\
\hline Selecting 2nd largest quality (\%) & 100 & 97.87 & 88.66 & 88.09 \\
\hline Average difference & 0.0012 & $9.785 \mathrm{E}-004$ & $7.457 \mathrm{E}-004$ & $6.047 \mathrm{E}-004$ \\
\hline Maximal difference & 0.0043 & 0.0044 & 0.0031 & 0.0027 \\
\hline Average relative difference (\%) & 0.12 & 0.1 & 0.077 & 0.062 \\
\hline Maximal relative difference (\%) & 0.45 & 0.47 & 0.32 & 0.28 \\
\hline
\end{tabular}

(a) $\delta_{\max }=0.1$

\begin{tabular}{|c|c|c|c|c|}
\hline$n$ & 3 & 4 & 5 & 6 \\
\hline Selecting 2nd largest quality (\%) & 100 & 89.86 & 78.26 & 78.27 \\
\hline Average difference & 0.0045 & 0.0035 & 0.0029 & 0.0022 \\
\hline Maximal difference & 0.0163 & 0.0133 & 0.0112 & 0.0110 \\
\hline Average relative difference (\%) & 0.50 & 0.38 & 0.31 & 0.24 \\
\hline Maximal relative difference (\%) & 1.78 & 1.44 & 1.21 & 1.19 \\
\hline
\end{tabular}

(b) $\delta_{\max }=0.2$

correctness of the proposition in Bernoulli-like case to be applicable drops. However, even when $\delta_{\max }=0.5$, still the majority of cases condition (4.57) is applicable to select optimal sequence (roughly more than $80 \%$ and $70 \%$ for $n=3$ and 6 , respectively). Similar results are obtained in subsequent analysis as well.

Based on the above, we conclude that condition (4.57) can be used as a criterion for selecting the sequence to maximize product quality.

\section{Sequence comparison under strictly sequencing and batch policies}

Under certain conditions, similar results to Proposition 4.5 under Bernoulli-relax assumption can be obtained. 
Proposition 4.9 Under assumptions (1)-(7) and Bernoulli-relax assumption (4.53), the following relationship holds for any sequence $s^{l}$ and $s^{m}, l, m \in\{1,2, \ldots,(n-1) !\}$

$$
P\left(g_{b t}^{l}\right)>P\left(g_{b t}^{m}\right) \quad \text { if and only if } P\left(g_{s s}^{l}\right)>P\left(g_{s s}^{m}\right)
$$

if

$$
0<\delta_{\max }<\frac{\widetilde{\mu}_{b t}^{l}-\widetilde{\mu}_{b t}^{m}}{\widetilde{\mu}_{b t}^{l}+\widetilde{\mu}_{b t}^{m}}=\frac{\widetilde{\mu}_{s s}^{l}-\widetilde{\mu}_{s s}^{m}}{\widetilde{\mu}_{s s}^{l}+\widetilde{\mu}_{s s}^{m}},
$$

where $\widetilde{\mu}_{s s}^{l}$ and $\widetilde{\mu}_{b t}^{l}$ are defined in 4.55) and 4.56), respectively.

Proof: See Appendix B.

However, condition (4.59) does not have a clear physical meaning and is difficult to apply. Therefore, we investigate the correctness of Proposition 4.5 under Bernoullirelax assumption numerically. For $\delta_{\max }=0.1$ and 0.2 , we consider number of products $n=3,4,5$, and 6 , and randomly and equiprobably select batch size $k_{i}$ from 2 to 6 for batch policy. Then we search the complete space of sequence options. For any given sequences $s^{l}$ and $s^{m}$, we compare the results of their relationships under both strictly sequencing and batch policies. If the comparison results are identical, it implies Proposition 4.5 is still correct under Bernoulli-relax assumption. The correctness of all the comparisons is summarized in Table 4.3. As one can see, for $\delta_{\max }=0.1$, within more than $98 \%$ of the cases, Proposition 4.5 still holds. When $\delta_{\max }=0.2$, such correctness is more than $96 \%$.

Table 4.3: Correctness of Proposition 4.5 under Bernoulli-relax assumption

\begin{tabular}{|c|c|c|c|c|}
\hline$n$ & 3 & 4 & 5 & 6 \\
\hline$\delta_{\max }=0.1$ & 98.42 & 98.23 & 98.16 & 98.08 \\
\hline$\delta_{\max }=0.2$ & 96.90 & 96.46 & 96.21 & 96.14 \\
\hline
\end{tabular}

Therefore, we formulate the following hypothesis: 
Hypothesis 4.1 Under assumptions (1)-(7) and when $\delta_{\max } \ll 1$, the following statement holds for any sequences $s^{l}$ and $s^{m}, l, m \in\{1,2, \ldots,(n-1) !\}$ :

$$
P\left(g_{b t}^{l}\right)>P\left(g_{b t}^{m}\right) \quad \text { if and only if } \quad P\left(g_{s s}^{l}\right)>P\left(g_{s s}^{m}\right)
$$

\section{Comparison between strictly sequencing and batch policies}

For the general case, we have

Proposition 4.10 Under assumptions (1)-(7) and Bernoulli-relax assumption 4.53), for equal batch size $k$, the following statements hold

(1) $P\left(g_{b t}^{l}\right)>P\left(\begin{array}{l}l \\ s s\end{array}\right)$ if $\mu_{n o-s w i t c h}^{\text {total }}>\mu_{\text {switch }}^{\text {total }}$ and

$$
0<\delta_{\text {max }}<\frac{\mu_{\text {no-switch }}^{\text {total }}-\mu_{\text {switch }}^{\text {total }}}{\mu_{\text {no-switch }}^{\text {total }}+\frac{k+1}{k-1} \mu_{\text {switch }}^{\text {total }}}
$$

(2) $P\left(g_{b t}^{l}\right)<P\left(g_{s s}^{l}\right)$ if $\mu_{\text {switch }}^{\text {total }}>\mu_{\text {no-switch }}^{\text {total }}$ and

$$
0<\delta_{\text {max }}<\frac{\mu_{\text {switch }}^{\text {total }}-\mu_{\text {no-switch }}^{\text {total }}}{\mu_{\text {no-switch }}^{\text {total }}+\frac{k+1}{k-1} \mu_{\text {switch }}^{\text {total }}} .
$$

Proof: See Appendix B.

Note that Proposition 4.10 can be reduced to Propositions 4.2 when $k=2$. As one can see, conditions in both propositions are similar to that in Propositions 4.6 and 4.7 in Bernoulli-like case. However, the exact conditions are difficult to apply in practice. Therefore, we carry out analysis numerically to verify whether Propositions 4.6 and 4.7 can be applied under Bernoulli-relax assumption or not. For the same sequence $s^{l}$, we consider number of product types $n=3$ to 6 , and randomly select batch size $k$ from 2 to 6 . Note that in this case, in order to keep the same percentage 
Table 4.4: Correctness of Proposition 4.6 under Bernoulli-relax assumption with identical $k_{i}$

\begin{tabular}{|c|c|c|c|c|}
\hline$n$ & 3 & 4 & 5 & 6 \\
\hline$\delta_{\max }=0.1$ & 98.14 & 98.24 & 98.10 & 98.01 \\
\hline$\delta_{\max }=0.2$ & 96.53 & 96.38 & 96.24 & 96.08 \\
\hline
\end{tabular}

of product composition under both policies, all $k_{i}=k$. The results are summarized in Table 4.4.

Clearly, more than $98 \%$ and $96 \%$ of cases for $\delta_{\max }=0.1$ and 0.2 obtain positive results, respectively. It implies that Proposition 4.6 can be applied under Bernoullirelax assumptions if $\delta_{\max }$ is small.

When $k_{i}$ s are not the same, we check the correctness of Proposition 4.7 under Bernoulli-relax assumption using the modified $\tilde{e}_{n o-s w i t c h}^{\text {total }}$ and $\widetilde{e}_{\text {switch }}^{\text {total }}$, the results are shown in Table 4.5

Table 4.5: Correctness of Proposition 4.7 under Bernoulli-relax assumption with random $k_{i}$

\begin{tabular}{|c|c|c|c|c|}
\hline$n$ & 3 & 4 & 5 & 6 \\
\hline$\delta_{\max }=0.1$ & 98.40 & 98.50 & 98.36 & 98.33 \\
\hline$\delta_{\max }=0.2$ & 96.81 & 96.90 & 96.81 & 96.74 \\
\hline
\end{tabular}

Again, we obtain the positive results with similar percentages. Therefore, Proposition 4.7 still holds in majority cases when $\delta_{\max }$ is small. Finally, we propose the following hypothesis:

Hypothesis 4.2 Under assumptions (1)-(7) and when $\delta_{\max } \ll 1$, with any batch 
size $k_{i}, \forall i$, the following statement holds:

$$
P\left(g_{b t}^{l}\right)>P\left(g_{s s}^{l}\right) \quad \text { if and only if } \tilde{e}_{n o-s w i t c h}^{\text {total }}>\widetilde{e}_{\text {switch }}^{\text {total }}
$$

\subsection{Quality Improvability with respect to Product Sequenc- ing}

Product sequencing has an impact on product quality. For example, in automotive paint shops, it is typical to sequence darker vehicles after the lighter one, and not vice versa, to improve quality. Thus, scheduling an appropriate sequence to achieve better quality is of significant importance. Developing a method to improve quality through resequencing, and to identify the worst sequence to avoid during operations, is necessary. Therefore, we define the improvability with respect to product sequencing as follows:

Definition 4.1 A flexible system with product sequence $s^{l}$ is improvable in terms of quality with respect to sequencing (referred to as $Q_{s}$ improvable) if there exists another sequence $s^{m}$ such that

$$
\sum_{s_{i}^{l}=1}^{n} \sum_{j=1}^{k_{s_{i}^{l}}} P\left(g_{s_{i}^{l}, j}\right)<\sum_{s_{i}^{m}=1}^{n} \sum_{j=1}^{k_{s_{i}^{m}}} P\left(g_{s_{i}^{m}, j}\right), \quad \forall m \in\{1,2, \ldots,(n-1) !\} .
$$

Otherwise it is unimprovable with respect to sequencing ( $Q_{s}$ unimprovable).

In this section, we seek $Q_{s}$ improvability indicators based on the collected data, rather than direct calculations of quality performance, to identify how to improve quality through resequencing. We pursue to use such indicators to compare the resulting system quality with different sequences.

In addition, we refer to the worst sequence in quality as the quality bottleneck sequence, which is defined as 
Definition 4.2 A quality bottleneck sequence (BN-s) is the one that satisfies

$$
\min _{l \in\{1,2, \ldots,(n-1) !\}}\left\{\sum_{s_{i}^{l}=1}^{n} \sum_{j=1}^{k_{s_{i}}} P\left(g_{s_{i}^{l}, j}\right)\right\} .
$$

Using a BN-s indicator, again based on the measured data on the factory floor, we can discover which sequence will be the bottleneck sequence that should be avoided in production operations.

In the case of strictly sequencing policy, i.e., $k_{i}=1, \forall i$, a system with sequence $s^{l}$ is $Q_{s}$ improvable if there exists another sequence $s^{m}$ such that

$$
\sum_{s_{i}^{l}=1}^{n} P\left(g_{s_{i}^{l}}\right)<\sum_{s_{i}^{m}=1}^{n} P\left(g_{s_{i}^{m}}\right) .
$$

The BN-s is the sequence that satisfies

$$
\min _{l \in\{1,2, \ldots,(n-1) !\}}\left\{\sum_{s_{i}^{l}=1}^{n} P\left(g_{s_{i}^{l}}\right)\right\} .
$$

To develop these indicators, we begin with the simple case under the Bernoulli-like assumption, then extend to more general cases. The results are presented below.

\subsubsection{Quality improvability under Bernoulli-like assumption}

As one can see from quality performance in batch and strictly sequencing policies

(Equations 4.42 and 4.43), only transitions between different products contribute to product quality (since $\sum_{i=1}^{n}\left(k_{s_{i}^{l}}-1\right) \mu_{s_{i}^{l}, s_{i}^{l}}$ are identical for all sequences). Thus, whether a system is $Q_{s}$ improvable or not can be answered by checking whether the summation of all repair probabilities with product switch is improvable or not. In other words, we have 
Proposition 4.11 Under assumptions (1)-(7) and the Bernoulli-like assumption (4.41), a flexible system with product sequence $s^{l}$ is $Q_{s}$ improvable if and only if there exists another sequence $s^{m}$ such that

$$
\begin{gathered}
\sum_{i=2}^{n} \mu_{s_{i}^{l}, s_{i-1}^{l}}+\mu_{s_{1}^{l}, s_{n}^{l}}<\sum_{i=2}^{n} \mu_{s_{i}^{m}, s_{i-1}^{m}}+\mu_{s_{1}^{m}, s_{n}^{m}}, \\
\forall m \in\{1,2, \ldots,(n-1) !\}, m \neq l .
\end{gathered}
$$

Otherwise, it is $Q_{s}$ unimprovable. In other words, $P\left(g^{l}\right)<P\left(g^{m}\right)$ if and only if $4.6^{7}$ ) holds.

Thus, $\sum_{i=2}^{n} \mu_{s_{i}^{l}, s_{i-1}^{l}}+\mu_{s_{1}^{l}, s_{n}^{l}}$ can be viewed as a $Q_{s}$ improvability indicator. Clearly, the optimal (i.e., $Q_{s}$ unimprovable) sequence is the one that leads to maximum $\sum_{i=2}^{n} \mu_{s_{i}^{l}, s_{i-1}^{l}}+\mu_{s_{1}^{l}, s_{n}^{l}}$.

The quality bottleneck sequence, i.e., the worst sequence in terms of quality, is then defined by the smallest summation of all repair probabilities with product switch. Therefore, an indicator for bottleneck sequence is introduced as:

$B N-s$ Indicator: Under assumptions (1)-(7) and the Bernoulli-like assumption (4.41), the quality bottleneck sequence is the one that satisfies

$$
\min _{l \in\{1,2, \ldots,(n-1) !\}}\left\{\sum_{i=2}^{n} \mu_{s_{i}^{l}, s_{i-1}^{l}}+\mu_{s_{1}^{l}, s_{n}^{l}}\right\} .
$$

As one can see, $\sum_{i=2}^{n} \mu_{s_{i}^{l}, s_{i-1}^{l}}+\mu_{s_{1}^{l}, s_{n}^{l}}$ can be used for $Q_{s}$ improvability and bottleneck sequence identifications from the point of view of product sequencing. In addition, such an indicator holds independently of batch or strictly sequencing policies. 


\subsubsection{Quality improvability under Bernoulli-relax assumption}

In practice, Bernoulli-like assumption does not have too much discrepancy from practical scenarios. However, such a condition is still too strict. Therefore, we prefer to relax this assumption to consider more general cases.

\section{Approximations of $P(g)$}

Under the Bernoulli-like assumption, a quality bottleneck sequence is the one with the smallest $\sum_{i=2}^{n} \mu_{s_{i}^{l}, s_{i-1}^{l}}+\mu_{s_{1}^{l}, s_{n}^{l}}$. Since $\mu_{i j}$ is identical to $e_{i j}$ under the Bernoullilike assumption, the sequence with the smallest $\sum_{i=2}^{n} e_{s_{i}^{l}, s_{i-1}^{l}}+e_{s_{1}^{l}, s_{n}^{l}}$ results in worst quality. In Bernoulli-relax scenario, the product quality will not only be dependent on $\mu_{i j}$, but also on $\lambda_{i j}$. Thus, some variations of indicator $\sum_{i=2}^{n} \mu_{s_{i}^{l}, s_{i-1}^{l}}+\mu_{s_{1}^{l}, s_{n}^{l}}$ need to be discovered for improvability and bottleneck identifications. We consider the possibility of developing an indicator by using $e_{i j}$. Specifically, we obtain:

Proposition 4.12 Under assumption (1)-(7) and the Bernoulli-relax assumption 4.53), for a given sequence $s^{l}$, the product quality can be evaluated as:

$$
\begin{aligned}
P\left(g_{b t}^{l}\right)= & \frac{\sum_{i=1}^{n}\left(k_{s_{i}^{l}}-1\right) e_{s_{i}^{l}, s_{i}^{l}}+\sum_{i=2}^{n} e_{s_{i}^{l}, s_{i-1}^{l}}+e_{s_{1}^{l}, s_{n}^{l}}}{K} \\
& +\frac{\left(\delta_{s_{1}^{l}, s_{1}^{l}}-\delta_{s_{1}^{l}, s_{n}^{l}}\right) e_{s_{1}^{l}, s_{n}^{l}}+\sum_{i=2}^{n-1}\left(\delta_{s_{i}^{l}, s_{i}^{l}}-\delta_{s_{i}^{l}, s_{i-1}^{l}}\right) e_{s_{i}^{l}, s_{i-1}^{l}}}{K\left(1-\delta_{s_{1}^{l}, s_{n}^{l}} \prod_{i=2}^{n} \delta_{s_{i}^{l}, s_{i-1}^{l}} \prod_{i=1}^{n} \delta_{s_{i}^{l}, s_{i}^{l}}^{k}\right)} \\
& +\frac{\left(\delta_{s_{n}^{l}, s_{n}^{l}}-\delta_{s_{n}^{l}, s_{n-1}^{l}}\right) e_{s_{n}^{l}, s_{n-1}^{l}}+\sum_{i=1}^{n-1}\left(\delta_{s_{i+1}^{l}, s_{i}^{l}}-\delta_{s_{i}^{l}, s_{i}^{l}}\right) e_{s_{i}^{l}, s_{i}^{l}}}{K\left(1-\delta_{s_{1}^{l}, s_{n}^{l}} \prod_{i=2}^{n} \delta_{s_{i}^{l}, s_{i-1}^{l}} \prod_{i=1}^{n} \delta_{s_{i}^{l}, s_{i}^{l}}^{l}\right)} \\
& +\frac{\left(\delta_{s_{1}^{l}, s_{n}^{l}}-\delta_{s_{n}^{l}, s_{n}^{l}}\right) e_{s_{n}^{l}, s_{n}^{l}}}{K\left(1-\delta_{s_{1}^{l}, s_{n}^{l}} \prod_{i=2}^{n} \delta_{s_{i}^{l}, s_{i-1}^{l}} \prod_{i=1}^{n} \delta_{s_{i}^{l}, s_{i}^{l}}^{k}\right)} \\
& +o\left(\delta_{\max }^{2}\right),
\end{aligned}
$$




$$
\begin{aligned}
P\left(g_{s s}^{l}\right)= & \frac{\sum_{i=2}^{n} e_{s_{i}^{l}, s_{i-1}^{l}}+e_{s_{1}^{l}, s_{n}^{l}}}{n} \\
& +\frac{\left(\delta_{s_{2}^{l}, s_{1}^{l}}-\delta_{s_{1}^{l}, s_{n}^{l}}\right) e_{s_{1}^{l}, s_{n}^{l}}+\sum_{i=2}^{n-1}\left(\delta_{s_{i+1}^{l}, s_{i}^{l}}-\delta_{s_{i}^{l}, s_{i-1}^{l}}\right) e_{s_{i}^{l}, s_{i-1}^{l}}+\left(\delta_{s_{1}^{l}, s_{n}^{l}}-\delta_{s_{n}^{l}, s_{n-1}^{l}}\right) e_{s_{n}^{l}, s_{n-1}^{l}}}{n\left(1-\delta_{s_{1}^{l}, s_{n}^{l}} \prod_{i=2}^{n} \delta_{s_{i}^{l}, s_{i-1}^{l}}\right)} \\
& +o\left(\delta_{\text {max }}^{2}\right) .
\end{aligned}
$$

Proof: See Appendix B,

In addition, with a relative loose approximation, we obtain

Proposition 4.13 Under assumption (1)-(7) and the Bernoulli-relax assumption (4.53), for a given sequence $s^{l}$, the product quality can be evaluated as:

$$
\begin{aligned}
P\left(g_{b t}^{l}\right) & =\frac{\sum_{i=1}^{n}\left(k_{s_{i}^{l}}-1\right) e_{s_{i}^{l}, s_{i}^{l}}+\sum_{i=2}^{n} e_{s_{i}^{l}, s_{i-1}^{l}}+e_{s_{1}^{l}, s_{n}^{l}}}{K}+o\left(\delta_{\text {max }}\right), \\
P\left(g_{s s}^{l}\right) & =\frac{\sum_{i=2}^{n} e_{s_{i}^{l}, s_{i-1}^{l}}+e_{s_{1}^{l}, s_{n}^{l}}}{n}+o\left(\delta_{\text {max }}\right) .
\end{aligned}
$$

Proof: See Appendix B,

Given product sequence $s^{l}$, by removing the terms of transitions within batches, and the denominators (assuming they are close to $K$ due to $\delta_{i j} \ll 1$ ), we define

$$
\begin{aligned}
I_{\delta_{\max }}^{l}= & \sum_{i=2}^{n} e_{s_{i}^{l}, s_{i-1}^{l}}+e_{s_{1}^{l}, s_{n}^{l}}, \\
I_{\delta_{\max }^{2}}^{l}= & \sum_{i=2}^{n} e_{s_{i}^{l}, s_{i-1}^{l}}+e_{s_{1}^{l}, s_{n}^{l}}+\sum_{i=1}^{n-1}\left(\delta_{s_{i+1}^{l}, s_{i}^{l}}-\delta_{s_{i}^{l}, s_{i}^{l}}\right) e_{s_{i}^{l}, s_{i}^{l}}+\left(\delta_{s_{1}^{l}, s_{n}^{l}}-\delta_{s_{n}^{l}, s_{n}^{l}}\right) e_{s_{n}^{l}, s_{n}^{l}} \\
& +\left(\delta_{s_{1}^{l}, s_{1}^{l}}-\delta_{s_{1}^{l}, s_{n}^{l}}\right) e_{s_{1}^{l}, s_{n}^{l}}+\sum_{i=2}^{n-1}\left(\delta_{s_{i}^{l}, s_{i}^{l}}-\delta_{s_{i}^{l}, s_{i-1}^{l}}\right) e_{s_{i}^{l}, s_{i-1}^{l}} \\
& +\left(\delta_{s_{n}^{l}, s_{n}^{l}}-\delta_{s_{n}^{l}, s_{n-1}^{l}}\right) e_{s_{n}^{l}, s_{n-1}^{l}} .
\end{aligned}
$$

We will investigate next whether $I_{\delta_{\max }}$ and $I_{\delta_{\max }^{2}}$ can be used as $Q_{s}$ improvability and bottleneck sequence indicators. 


\section{Indicator $I_{\delta_{\max }^{2}}$}

First we consider using $I_{\delta_{\max }^{2}}$ as an indicator for improvability and bottleneck identifications. We expect to identify the $Q_{s}$ improvability using $I_{\delta_{\max }^{2}}$, i.e.,

$$
I_{\delta_{\max }^{2}}^{l}>I_{\delta_{\max }^{2}}^{m} \quad \text { if and only if } P\left(g^{l}\right)>P\left(g^{m}\right)
$$

The unimprovable (or, optimal) sequence and the bottleneck sequence can also be

discovered by finding the ones which maximize and minimize $I_{\delta_{\text {max }}^{2}}$, respectively. To check the correctness of such an indicator, we investigate the bound for $o\left(\delta_{\max }^{2}\right)$ first. Let function $\operatorname{sum}(\cdot)$ represent the summation of the elements of a vector, i.e., for $\rho=\left[\rho_{1}, \rho_{2}, \ldots, \rho_{n}\right]^{T}$,

$$
\operatorname{sum}(\rho)=\sum_{i=1}^{n} \rho_{i}
$$

Then we obtain

Corollary 4.2 Under assumption (1)-(7) and the Bernoulli-relax assumption (4.53), for a given sequence $s^{l}$, the following bound is obtained:

$$
\left|o\left(\delta_{\max }^{2}\right)\right| \leq 2\left(\frac{1}{1-\delta_{\max }}-\frac{1+\delta_{\max }}{1-\delta_{\max }^{K}}\right) \operatorname{sum}\left(\frac{1}{K} E\right)
$$

where the vector $E$ is defined as

$$
E=\left[e_{s_{1}^{l}, s_{n}^{l}}, e_{s_{1}^{l}, s_{1}^{l}}, \ldots, e_{s_{1}^{l}, s_{1}^{l}}, e_{s_{2}^{l}, s_{1}^{l}}, e_{s_{2}^{l}, s_{2}^{l}}, \ldots, e_{s_{n}^{l}, s_{n-1}^{l}}, e_{s_{n}^{l}, s_{n}^{l}}, \ldots, e_{s_{n}^{l}, s_{n}^{l}}\right]^{T}
$$

for batch policy, and

$$
E=\left[e_{s_{1}^{l}, s_{n}^{l}}, e_{s_{2}^{l}, s_{1}^{l}}, \ldots, e_{s_{n}^{l}, s_{n-1}^{l}}\right]^{T}
$$

for strictly sequencing policy. 
Proof: See Appendix B.

As one can expect, when $\delta_{\max }$ is small, such a bound will also be small, which implies that most likely Indicator $I_{\delta_{\max }^{2}}$ leads to an accurate identification. To further investigate this accuracy, numerical experiments are carried out by randomly selecting $\delta_{i j}$. For any given $\delta_{\max }$, we randomly and equiprobably select $\mu_{i j}$ and then $\lambda_{i j}$ such that

$$
\begin{aligned}
& \lambda_{i j} \in\left(0, \delta_{\max }\right], \\
& \mu_{i j} \in\left[1-\delta_{\max }, 1\right),
\end{aligned}
$$

Such a selection implies that

$$
1-\delta_{\max }<\lambda_{i j}+\mu_{i j}<1+\delta_{\max }
$$

In addition, batch size $k_{i}$ is also selected randomly from

$$
k_{i} \in\{1,2, \ldots, 6\}
$$

Then, we investigate whether condition 4.75 holds or not under the Bernoullirelax assumption. For a selected $\delta_{\max }$, we randomly generate two sequences. We calculate the quality efficiencies and evaluate the good job probabilities for both sequences. Then we compare the two sequences based on Indicator $I_{\delta_{\max }^{2}}$ (i.e., using condition (4.75) and the good job probabilities. If both comparisons result in identical conclusions, it implies that condition 4.75 is correct. Otherwise an incorrect comparison is obtained. For the cases of incorrect comparison, we check the differences in quality between these two sequences. Such experiments are repeated for 10,000 times. 
The results of this experiment are summarized in Tables 4.6 and 4.7. It can be seen from Tables 4.6 that condition 4.75 is correct almost for all cases (larger than 99\%) when $\delta_{\max }=0.1$ and 0.2 . For the cases where condition 4.75 results in incorrect comparisons, let $s^{l}$ and $s^{m}$ are two sequences under comparison, and $P\left(g^{l}\right)$ and $P\left(g^{m}\right)$ are the corresponding quality. When the comparison results are different, define

$$
\begin{aligned}
\Delta_{c} & =\left|P\left(g^{l}\right)-P\left(g^{m}\right)\right| \\
\epsilon_{c} & =\frac{\left|P\left(g^{l}\right)-P\left(g^{m}\right)\right|}{\frac{P\left(g^{l}\right)+P\left(g^{m}\right)}{2}} \cdot 100 \% .
\end{aligned}
$$

Then, it can be seen from Table 4.7 that, in this case, the differences in quality between these two sequences are extremely small. Thus, we conclude that Indicator $I_{\delta_{\max }^{2}}$ (i.e., condition 4.75) can provide an effective comparison between two sequences.

Table 4.6: Effectiveness of Indicator $I_{\delta_{\max }^{2}}$ for $Q_{s}$ improvability (\%)

\begin{tabular}{|c|c|c|c|c|}
\hline$n$ & 3 & 4 & 5 & 6 \\
\hline$\delta_{\max }=0.1$ & 99.94 & 99.90 & 99.94 & 99.92 \\
\hline$\delta_{\max }=0.2$ & 99.62 & 99.67 & 99.68 & 99.74 \\
\hline
\end{tabular}

To identify the effectiveness of Indicator $I_{\delta_{\max }^{2}}$ for $Q_{s}$ unimprovable sequence, the optimal sequences selected by complete search of the possible combinations are compared with the sequences chosen using Indicator $I_{\delta_{\max }^{2}}\left(\right.$ i.e., $\left.\max _{l} I_{\delta_{\max }^{2}}^{l}\right)$. When both the chosen sequences are identical, the indicator results in an effective selection. The percentages of correct selections under different scenarios are summarized in Table 4.8 for $\delta_{\max }=0.1$ and 0.2 . It is clear that when $\delta_{\max }$ is small, which is the scenario of majority cases, such an indicator results in a high percentage of correctness (about $99 \%$ or more). 
Table 4.7: Errors when Indicator $I_{\delta_{\text {max }}^{2}}$ leads to incorrect comparisons

\begin{tabular}{|c|c|c|c|c|}
\hline$n$ & 3 & 4 & 5 & 6 \\
\hline Average $\Delta_{c}$ & $1.91 \mathrm{E}-05$ & $9.67 \mathrm{E}-06$ & $4.01 \mathrm{E}-06$ & $8.12 \mathrm{E}-06$ \\
\hline Maximal $\Delta_{c}$ & $3.74 \mathrm{E}-05$ & $2.75 \mathrm{E}-05$ & $1.02 \mathrm{E}-05$ & $3.03 \mathrm{E}-05$ \\
\hline Average $\epsilon_{c}(\%)$ & $2.02 \mathrm{E}-03$ & $1.02 \mathrm{E}-03$ & $4.18 \mathrm{E}-04$ & $8.54 \mathrm{E}-04$ \\
\hline Maximal $\epsilon_{c}(\%)$ & $3.98 \mathrm{E}-03$ & $2.89 \mathrm{E}-03$ & $1.06 \mathrm{E}-03$ & $3.21 \mathrm{E}-03$ \\
\hline
\end{tabular}

(a) $\delta_{\max }=0.1$

\begin{tabular}{|c|c|c|c|c|}
\hline$n$ & 3 & 4 & 5 & 6 \\
\hline Average $\Delta_{c}$ & $1.90 \mathrm{E}-04$ & $1.11 \mathrm{E}-04$ & $8.63 \mathrm{E}-05$ & $6.49 \mathrm{E}-05$ \\
\hline Maximal $\Delta_{c}$ & $1.29 \mathrm{E}-03$ & $5.42 \mathrm{E}-04$ & $3.87 \mathrm{E}-04$ & $3.36 \mathrm{E}-04$ \\
\hline Average $\epsilon_{c}(\%)$ & $2.12 \mathrm{E}-02$ & $1.23 \mathrm{E}-02$ & $9.63 \mathrm{E}-03$ & $7.24 \mathrm{E}-03$ \\
\hline Maximal $\epsilon_{c}(\%)$ & $1.45 \mathrm{E}-01$ & $6.07 \mathrm{E}-02$ & $4.34 \mathrm{E}-02$ & $3.74 \mathrm{E}-02$ \\
\hline
\end{tabular}

(b) $\delta_{\max }=0.2$

Again we investigate the cases where Indicator $I_{\delta_{\max }^{2}}$ does not select the optimal sequence. In such scenario, a sub-optimal one is typically chosen. In Table 4.9, the cases of incorrect selections are analyzed. First, the percentage that Indicator $I_{\delta_{\max }^{2}}$ selects the sequence with the second largest quality is presented. It can be seen that among all the incorrectly chosen cases, roughly all of them result in selecting the sequence with the second large quality based on Indicator $I_{\delta_{\max }^{2}}$. Moreover, we investigate the differences in quality between the optimal sequence and the one selected using Indicator $I_{\delta_{\max }^{2}}$. Define

$$
\begin{aligned}
\Delta_{o} & =\left|P\left(g^{l}\right)-P\left(g^{o p t}\right)\right| \\
\epsilon_{o} & =\frac{\left|P\left(g^{l}\right)-P\left(g^{o p t}\right)\right|}{P\left(g^{o p t}\right)} \cdot 100 \% .
\end{aligned}
$$

As one can see, such differences are extremely small.

Finally, we check the correctness of Indicator $I_{\delta_{\max }^{2}}$ for bottleneck identification (i.e., $\left.\min _{l} I_{\delta_{\max }^{2}}^{l}\right)$. As shown in Tables 4.10 and 4.11, it can correctly identify the 
Table 4.8: Effectiveness of Indicator $I_{\delta_{\max }^{2}}$ for $Q_{s}$ unimprovable sequence (\%)

\begin{tabular}{|c|c|c|c|c|}
\hline $\mathrm{n}$ & 3 & 4 & 5 & 6 \\
\hline$\delta_{\max }=0.1$ & 99.92 & 99.90 & 99.69 & 99.67 \\
\hline$\delta_{\max }=0.2$ & 99.66 & 99.31 & 98.92 & 98.35 \\
\hline
\end{tabular}

Table 4.9: Errors when Indicator $I_{\delta_{\max }^{2}}$ identifies incorrect unimprovable sequence

\begin{tabular}{|c|c|c|c|c|}
\hline $\mathrm{n}$ & 3 & 4 & 5 & 6 \\
\hline Selecting 2nd largest quality $(\%)$ & 100 & 100 & 100 & 100 \\
\hline Average $\Delta_{o}$ & $1.44 \mathrm{E}-05$ & $1.74 \mathrm{E}-05$ & $1.07 \mathrm{E}-05$ & $1.50 \mathrm{E}-05$ \\
\hline Maximal $\Delta_{o}$ & $8.31 \mathrm{E}-05$ & $6.59 \mathrm{E}-05$ & $5.58 \mathrm{E}-05$ & $4.14 \mathrm{E}-05$ \\
\hline Average $\epsilon_{o}(\%)$ & $1.51 \mathrm{E}-03$ & $1.82 \mathrm{E}-03$ & $1.13 \mathrm{E}-03$ & $1.57 \mathrm{E}-03$ \\
\hline Maximal $\epsilon_{o}(\%)$ & $8.67 \mathrm{E}-03$ & $6.80 \mathrm{E}-03$ & $5.89 \mathrm{E}-03$ & $4.36 \mathrm{E}-03$ \\
\hline
\end{tabular}

(a) $\delta_{\max }=0.1$

\begin{tabular}{|c|c|c|c|c|}
\hline $\mathrm{n}$ & 3 & 4 & 5 & 6 \\
\hline Selecting 2nd largest quality $(\%)$ & 100 & 98.55 & 95.37 & 95.15 \\
\hline Average $\Delta_{o}$ & $1.49 \mathrm{E}-04$ & $1.47 \mathrm{E}-04$ & $1.35 \mathrm{E}-04$ & $1.17 \mathrm{E}-04$ \\
\hline Maximal $\Delta_{o}$ & $3.94 \mathrm{E}-04$ & $5.46 \mathrm{E}-04$ & $9.11 \mathrm{E}-04$ & $5.09 \mathrm{E}-04$ \\
\hline Average $\epsilon_{o}(\%)$ & $1.67 \mathrm{E}-02$ & $1.61 \mathrm{E}-02$ & $1.47 \mathrm{E}-02$ & $1.28 \mathrm{E}-02$ \\
\hline Maximal $\epsilon_{o}(\%)$ & $4.45 \mathrm{E}-02$ & $6.00 \mathrm{E}-02$ & $9.91 \mathrm{E}-02$ & $5.43 \mathrm{E}-02$ \\
\hline
\end{tabular}

(b) $\delta_{\max }=0.2$ 
bottleneck sequence in almost all cases. Even if the identification is not correct, the sequence with the second worst quality is typically identified and the differences in quality comparing with the bottleneck one are extremely small. In Table 4.11, $\Delta_{b}$ and $\epsilon_{b}$ are defined as:

$$
\begin{aligned}
\Delta_{b} & =\left|P\left(g^{l}\right)-P\left(g^{b}\right)\right|, \\
\epsilon_{b} & =\frac{\left|P\left(g^{l}\right)-P\left(g^{b}\right)\right|}{P\left(g^{b}\right)} \cdot 100 \% .
\end{aligned}
$$

Table 4.10: Effectiveness of BN-s Indicator $I_{\delta_{\max }^{2}}(\%)$

\begin{tabular}{|c|c|c|c|c|}
\hline $\mathrm{n}$ & 3 & 4 & 5 & 6 \\
\hline$\delta_{\max }=0.1$ & 99.93 & 99.88 & 99.72 & 99.65 \\
\hline$\delta_{\max }=0.2$ & 99.66 & 99.38 & 98.86 & 98.77 \\
\hline
\end{tabular}

Table 4.11: Errors when Indicator $I_{\delta_{\max }^{2}}$ results in incorrect BN-s identification

\begin{tabular}{|c|c|c|c|c|}
\hline $\mathrm{n}$ & 3 & 4 & 5 & 6 \\
\hline Selecting 2nd largest quality (\%) & 100 & 100 & 100 & 96.43 \\
\hline Average $\Delta_{b}$ & $1.39 \mathrm{E}-05$ & $1.52 \mathrm{E}-05$ & $1.45 \mathrm{E}-05$ & $9.81 \mathrm{E}-06$ \\
\hline Maximal $\Delta_{b}$ & $3.64 \mathrm{E}-05$ & $5.90 \mathrm{E}-05$ & $5.10 \mathrm{E}-05$ & $3.94 \mathrm{E}-05$ \\
\hline Average $\epsilon_{b}(\%)$ & $1.47 \mathrm{E}-03$ & $1.60 \mathrm{E}-03$ & $1.51 \mathrm{E}-03$ & $1.02 \mathrm{E}-03$ \\
\hline Maximal $\epsilon_{b}(\%)$ & $3.89 \mathrm{E}-03$ & $6.19 \mathrm{E}-03$ & $5.31 \mathrm{E}-03$ & $4.08 \mathrm{E}-03$ \\
\hline
\end{tabular}

(a) $\delta_{\max }=0.1$

\begin{tabular}{|c|c|c|c|c|}
\hline $\mathrm{n}$ & 3 & 4 & 5 & 6 \\
\hline Selecting 2nd largest quality (\%) & 100 & 99.12 & 96.77 & 96.75 \\
\hline Average $\Delta_{b}$ & $2.17 \mathrm{E}-04$ & $1.24 \mathrm{E}-04$ & $9.79 \mathrm{E}-05$ & $1.20 \mathrm{E}-04$ \\
\hline Maximal $\Delta_{b}$ & $9.78 \mathrm{E}-04$ & $8.29 \mathrm{E}-04$ & $4.17 \mathrm{E}-04$ & $6.18 \mathrm{E}-04$ \\
\hline Average $\epsilon_{b}(\%)$ & $2.40 \mathrm{E}-02$ & $1.35 \mathrm{E}-02$ & $1.07 \mathrm{E}-02$ & $1.31 \mathrm{E}-02$ \\
\hline Maximal $\epsilon_{b}(\%)$ & $1.10 \mathrm{E}-01$ & $8.83 \mathrm{E}-02$ & $4.54 \mathrm{E}-02$ & $6.95 \mathrm{E}-02$ \\
\hline
\end{tabular}

(b) $\delta_{\max }=0.2$

Based on the above results, we proposed the following hypothesis: 
Hypothesis 4.3 Under assumptions (1)-(7) and the Bernoulli-relax assumption (4.53), a flexible system with product sequence $s^{l}$ is $Q_{s}$ improvable if and only if there exists another sequence $s^{m}$ such that

$$
I_{\delta_{\text {max }}^{2}}^{l}<I_{\delta_{\text {max }}^{2}}^{m}, \quad \forall m \in\{1,2, \ldots,(n-1) !\}, m \neq l .
$$

Otherwise, it is $Q_{s}$ unimprovable. In other words, $P\left(g^{l}\right)<P\left(g^{m}\right)$ if and only if (4.77) holds.

In addition, we propose the following $\mathrm{BN}-\mathrm{s}$ indicator:

$B N-s$ Indicator: Under assumptions (1)-(7) and the Bernoulli-relax assumption (4.53), the quality bottleneck sequence is the one that satisfies

$$
\min _{l \in\{1,2, \ldots,(n-1) !\}} I_{\delta_{\max }^{2}}^{l}
$$

Although Indicator $I_{\delta_{\max }^{2}}$ can lead to effective identification of $Q_{s}$ improvability and bottleneck sequence, its computation is relatively complicated. To make the indicator simple, we investigate Indicator $I_{\delta_{\max }}$ next.

\section{Indicator $I_{\delta_{\max }}$}

Now we expect to identify the $Q_{s}$ improvability using $I_{\delta_{\max }}$, i.e.,

$$
I_{\delta_{\max }}^{l}>I_{\delta_{\max }^{m}}^{m} \text { if and only if } P\left(g^{l}\right)>P\left(g^{m}\right)
$$

Then, the maximal and minimal $I_{\delta_{\max }}$ can be used to identify the unimprovable and the bottleneck sequences, respectively. In addition to the rationale from Equations (4.42) and 4.43), Indicator $I_{\delta_{\max }}$ can also be understood by directly replacing $\mu_{i j}$ in Proposition 4.11 with $e_{i j}$, where under Bernoulli-like assumption, they are identical. 
Intuitively we expect such replacement will lead to effective identification in most cases.

First, we evaluate the bound for $o\left(\delta_{\max }\right)$.

Corollary 4.3 Under assumption (1)-(7) and the Bernoulli-relax assumption (4.53), for a given sequence $s^{l}$, the following bound is obtained:

$$
\left|o\left(\delta_{\max }\right)\right| \leq 2\left(\frac{1}{1-\delta_{\max }}-\frac{1}{1-\delta_{\max }^{K}}\right) \operatorname{sum}\left(\frac{1}{K} E\right)
$$

where the vector $E$ is defined as

$$
E=\left[e_{s_{1}^{l}, s_{n}^{l}}, e_{s_{1}^{l}, s_{1}^{l}}, \ldots, e_{s_{1}^{l}, s_{1}^{l}}, e_{s_{2}^{l}, s_{1}^{l}}, e_{s_{2}^{l}, s_{2}^{l}}, \ldots, e_{s_{n}^{l}, s_{n-1}^{l}}, e_{s_{n}^{l}, s_{n}^{l}}, \ldots, e_{s_{n}^{l}, s_{n}^{l}}\right]^{T}
$$

for batch policy, and

$$
E=\left[e_{s_{1}^{l}, s_{n}^{l}}, e_{s_{2}^{l}, s_{1}^{l}}, \ldots, e_{s_{n}^{l}, s_{n-1}^{l}}\right]^{T}
$$

for strictly sequencing policy.

Proof: See Appendix B.

Thus, although $o\left(\delta_{\max }\right)$ is inferior to $o\left(\delta_{\max }^{2}\right)$, its bound is still small when $\delta_{\max }$ is small. Therefore, similar numerical experiments are carried out to further investigate the effectiveness of Indicator $I_{\delta_{\max }}$. The results for $Q_{s}$ improvability identification are summarized in Table 4.12 and 4.13. It can be seen from Table 4.12 that Indicator $I_{\delta_{\max }}$ (i.e., condition 4.79$)$ ) is correct for more than $98 \%$ and $96 \%$ for $\delta_{\max }=0.1$ and 0.2 , respectively. For the scenarios where condition 4.79 results in incorrect comparisons, it can be seen from Table 4.13 that, in this case, the differences in quality between two sequences are very small. Thus, we conclude that Indicator $I_{\delta_{\max }}$ can provide an effective comparison between two sequences. 
Table 4.12: Effectiveness of Indicator $I_{\delta_{\max }}$ for $Q_{s}$ improvability (\%)

\begin{tabular}{|c|c|c|c|c|}
\hline$n$ & 3 & 4 & 5 & 6 \\
\hline$\delta_{\max }=0.1$ & 98.21 & 98.35 & 98.13 & 98.12 \\
\hline$\delta_{\max }=0.2$ & 98.18 & 96.52 & 96.05 & 96.20 \\
\hline
\end{tabular}

Table 4.13: Errors when Indicator $I_{\delta_{\max }}$ leads to incorrect comparisons

\begin{tabular}{|c|c|c|c|c|}
\hline$n$ & 3 & 4 & 5 & 6 \\
\hline Average $\Delta_{c}$ & $3.80 \mathrm{E}-04$ & $2.55 \mathrm{E}-04$ & $2.37 \mathrm{E}-04$ & $1.98 \mathrm{E}-04$ \\
\hline Maximal $\Delta_{c}$ & $3.01 \mathrm{E}-03$ & $2.13 \mathrm{E}-03$ & $1.63 \mathrm{E}-03$ & $1.14 \mathrm{E}-03$ \\
\hline Average $\epsilon_{c}(\%)$ & $4.00 \mathrm{E}-02$ & $2.68 \mathrm{E}-02$ & $2.50 \mathrm{E}-02$ & $2.08 \mathrm{E}-02$ \\
\hline Maximal $\epsilon_{c}(\%)$ & 0.31 & 0.23 & 0.17 & 0.12 \\
\hline
\end{tabular}

(a) $\delta_{\max }=0.1$

\begin{tabular}{|c|c|c|c|c|}
\hline$n$ & 3 & 4 & 5 & 6 \\
\hline Average $\Delta_{c}$ & $1.37 \mathrm{E}-03$ & $1.01 \mathrm{E}-03$ & $8.96 \mathrm{E}-04$ & $8.76 \mathrm{E}-04$ \\
\hline Maximal $\Delta_{c}$ & $1.20 \mathrm{E}-02$ & $8.85 \mathrm{E}-03$ & $7.98 \mathrm{E}-03$ & $8.70 \mathrm{E}-03$ \\
\hline Average $\epsilon_{c}(\%)$ & 0.15 & 0.11 & $9.95 \mathrm{E}-02$ & $9.71 \mathrm{E}-02$ \\
\hline Maximal $\epsilon_{c}(\%)$ & 1.30 & 0.99 & 0.87 & 0.94 \\
\hline
\end{tabular}

(b) $\delta_{\max }=0.2$ 
Similar results are obtained to validate that the maximal value of $I_{\delta_{\max }}$ leads to $Q_{s}$ unimprovable (i.e., optimal) sequence. It is shown in Table 4.14 and 4.15 that the correctness of using indicator is more than $98 \%$ for $n=3$ and $93 \%$ for $n=6$ when $\delta_{\max }=0.1$, and $96 \%$ for $n=3$ and $85 \%$ for $n=6$ when $\delta_{\max }=0.2$. In addition, it typically identifies the second largest quality and the difference (in quality) compared with the optimal sequence is very small.

Table 4.14: Effectiveness of Indicator $I_{\delta_{\max }}$ for $Q_{s}$ unimprovable sequence (\%)

\begin{tabular}{|c|c|c|c|c|}
\hline $\mathrm{n}$ & 3 & 4 & 5 & 6 \\
\hline$\delta_{\max }=0.1$ & 98.08 & 96.26 & 94.53 & 92.22 \\
\hline$\delta_{\max }=0.2$ & 96.22 & 92.37 & 88.15 & 84.89 \\
\hline
\end{tabular}

Table 4.15: Errors when Indicator $I_{\delta_{\max }}$ identifies incorrect unimprovable sequence

\begin{tabular}{|c|c|c|c|c|}
\hline $\mathrm{n}$ & 3 & 4 & 5 & 6 \\
\hline Selecting 2nd largest quality $(\%)$ & 100 & 94.65 & 89.40 & 86.50 \\
\hline Average $\Delta_{o}$ & $6.38 \mathrm{E}-04$ & $5.56 \mathrm{E}-04$ & $5.48 \mathrm{E}-04$ & $5.24 \mathrm{E}-04$ \\
\hline Maximal $\Delta_{o}$ & $3.42 \mathrm{E}-03$ & $2.96 \mathrm{E}-03$ & $3.05 \mathrm{E}-03$ & $2.56 \mathrm{E}-03$ \\
\hline Average $\epsilon_{o}(\%)$ & $6.72 \mathrm{E}-02$ & $5.82 \mathrm{E}-02$ & $5.72 \mathrm{E}-02$ & $5.47 \mathrm{E}-02$ \\
\hline Maximal $\epsilon_{o}(\%)$ & 0.36 & 0.31 & 0.32 & 0.27 \\
\hline
\end{tabular}

(a) $\delta_{\max }=0.1$

\begin{tabular}{|c|c|c|c|c|}
\hline $\mathrm{n}$ & 3 & 4 & 5 & 6 \\
\hline Selecting 2nd largest quality $(\%)$ & 100 & 91.74 & 76.29 & 73.59 \\
\hline Average $\Delta_{o}$ & $2.16 \mathrm{E}-03$ & $2.11 \mathrm{E}-03$ & $2.00 \mathrm{E}-03$ & $1.89 \mathrm{E}-03$ \\
\hline Maximal $\Delta_{o}$ & $1.09 \mathrm{E}-02$ & $1.43 \mathrm{E}-02$ & $1.11 \mathrm{E}-02$ & $9.98 \mathrm{E}-03$ \\
\hline Average $\epsilon_{o}(\%)$ & 0.24 & 0.23 & 0.22 & 0.21 \\
\hline Maximal $\epsilon_{o}(\%)$ & 1.16 & 1.57 & 1.20 & 1.10 \\
\hline
\end{tabular}

(b) $\delta_{\max }=0.2$

Although the accuracy of Indicator $I_{\delta_{\max }}$ is not as good as that of Indicator $I_{\delta_{\max }^{2}}$, it is still relative high and acceptable for practical applications. More important, the calculation is much simpler and is more intuitive. Therefore, another hypothesis is proposed below: 
Hypothesis 4.4 Under assumptions (1)-(7) and the Bernoulli-relax assumption (4.53), a flexible system with product sequence $s^{l}$ is $Q_{s}$ improvable if and only if there exists another sequence $s^{m}$ such that

$$
I_{\delta_{\max }}^{l}<I_{\delta_{\max }}^{m}, \quad \forall m \in\{1,2, \ldots,(n-1) !\}, m \neq l
$$

Otherwise, it is $Q_{s}$ unimprovable. In other words, $P\left(g^{l}\right)<P\left(g^{m}\right)$ if and only if (4.81) holds.

Next we study bottleneck sequence. As shown in Table 4.16, when $\delta_{\max }$ is small, which is the scenario of majority cases in practice, Indicator $I_{\delta_{\max }}$ results in a relative high percentage of correctness, typically more than $90 \%$. For the incorrectly identified cases, Table 4.17 suggests that the sequence with the second worst quality is typically calculated. Roughly $90 \%$ and $80 \%$ of them result in selecting the sequences with the second worst quality, for $\delta_{\max }=0.1$ and 0.2 , respectively. Moreover, the differences in quality are also very small.

Table 4.16: Effectiveness of BN-s Indicator $I_{\delta_{\max }}(\%)$

\begin{tabular}{|c|c|c|c|c|}
\hline $\mathrm{n}$ & 3 & 4 & 5 & 6 \\
\hline$\delta_{\max }=0.1$ & 98.14 & 96.26 & 94.14 & 92.19 \\
\hline$\delta_{\max }=0.2$ & 96.54 & 92.03 & 88.65 & 84.99 \\
\hline
\end{tabular}

Remark 4.8 In addition to the above cases where $\delta_{\max }$ is small, for completeness of the study, we also investigate the cases when $\delta_{\max }$ is large. As one expects, the percentage of correctness of BN-s indicator using Indicator $I_{\delta_{\max }}$ drops. However, even when $\delta_{\max }=0.5$, still the percentages that Indicator $I_{\delta_{\max }}$ can select the correct bottleneck sequence are more than $90 \%$ and $65 \%$ for $n=3$ and 6 , respectively. 
Table 4.17: Errors when Indicator $I_{\delta_{\max }}$ results in incorrect BN-s identification

\begin{tabular}{|c|c|c|c|c|}
\hline $\mathrm{n}$ & 3 & 4 & 5 & 6 \\
\hline Selecting 2nd largest quality $(\%)$ & 100 & 95.72 & 92.49 & 88.99 \\
\hline Average $\Delta_{b}$ & $5.75 \mathrm{E}-04$ & $5.24 \mathrm{E}-04$ & $5.00 \mathrm{E}-04$ & $4.70 \mathrm{E}-04$ \\
\hline Maximal $\Delta_{b}$ & $2.52 \mathrm{E}-03$ & $2.69 \mathrm{E}-03$ & $2.81 \mathrm{E}-03$ & $2.39 \mathrm{E}-03$ \\
\hline Average $\epsilon_{b}(\%)$ & $6.05 \mathrm{E}-02$ & $5.48 \mathrm{E}-02$ & $5.22 \mathrm{E}-02$ & $4.90 \mathrm{E}-02$ \\
\hline Maximal $\epsilon_{b}(\%)$ & 0.26 & 0.28 & 0.29 & 0.25 \\
\hline
\end{tabular}

(a) $\delta_{\max }=0.1$

\begin{tabular}{|c|c|c|c|c|}
\hline $\mathrm{n}$ & 3 & 4 & 5 & 6 \\
\hline Selecting 2nd largest quality $(\%)$ & 100 & 90.46 & 82.64 & 76.28 \\
\hline Average $\Delta_{b}$ & $2.42 \mathrm{E}-03$ & $1.82 \mathrm{E}-03$ & $1.83 \mathrm{E}-03$ & $1.69 \mathrm{E}-03$ \\
\hline Maximal $\Delta_{b}$ & $1.56 \mathrm{E}-02$ & $1.13 \mathrm{E}-02$ & $9.82 \mathrm{E}-03$ & $1.04 \mathrm{E}-02$ \\
\hline Average $\epsilon_{b}(\%)$ & 0.27 & 0.20 & 0.20 & 0.18 \\
\hline Maximal $\epsilon_{b}(\%)$ & 1.70 & 1.23 & 1.06 & 1.17 \\
\hline
\end{tabular}

(b) $\delta_{\max }=0.2$

Based on the above, we conclude that Indicator $I_{\delta_{m a x}}$ can be used as a criterion to identify bottleneck sequence.

$B N-s$ Indicator: Under assumptions (1)-(7) and the Bernoulli-relax assumption (4.53), the quality bottleneck sequence is the one that satisfies

$$
\min _{l \in\{1,2, \ldots,(n-1) !\}} I_{\delta_{\max }^{l}}^{l}
$$

\subsection{Summary}

In this chapter, an analytical method based on Markov chain model is presented to study the product sequence policy with respect to the quality performance in a flexible manufacturing system with batch productions. In addition, we investigate the impact of product sequencing on quality. The optimal sequences leading to best quality under both batch and strict sequencing policies are obtained, the comparisons between 
different sequences under both policies are carried out, and finally the comparison between two policies is studied. It is shown that the product sequence observes the same characteristics under both policies (e.g., the optimality and comparison results), and batch policies outperforms the strictly sequencing policy if the total quality efficiency without product switch is better than the total quality efficiency with product switch. The model introduced here can be used to evaluate quality and investigate sequencing policies in many flexible systems with batch operations. It can also provide guidance for system design and operation to achieve better quality. As introduced in the application example, significant improvement in quality can be obtained by selecting a better sequencing and batch policy. However, it still has limitations. One of the limitation of this method is that it requires substantial amount of data to obtain stable results on transition probabilities. It is typically more suitable for manufacturing systems with $100 \%$ inspections, or for systems with sampling inspections but having a necessary quality data to calculate transition probabilities. Another limitation is related to the computation efficiency when the number of products is large. In this case, the dimension of the matrix will be increasing significantly.

In addition, $Q_{s}$ improvability and quality bottleneck sequence are defined. A flexible system is $Q_{s}$ improvable if there exists another sequence which renders better quality, and a quality bottleneck sequence is the one that impedes quality in the strongest manner. To check $Q_{s}$ improvability or to identify a quality bottleneck sequence, indicators based on collected data on the factory floor are developed. Such development provides a simple tool for production engineers and managers to design the appropriate sequence to achieve higher quality in flexible manufacturing systems. 


\section{CHAPTER 5}

\section{BOTTLENECK TRANSITION WITH RESPECT TO QUALITY}

As shown in Equations (4.36) and (4.40), the product quality is a function of the transition probabilities, such as $\lambda_{i j}$ and $\mu_{i j}$. Thus, improving these transition probabilities could lead to improvement of product quality. The question is, which transition should we focus on? To improve product quality most efficiently, the transition, whose improvement will lead to the largest improvement in product quality comparing with improving all other transitions, should be the one. Such a transition is referred to as quality bottleneck transition, which impedes the product quality in the strongest manner. Therefore, the bottleneck transition has the largest impact on quality.

Based on the above, we define

Definition 5.1 Under assumptions (1)-(7), transition probability $\lambda_{i j}$ is the negative bottleneck transition ( $n$ - $B N-t$ ) with respect to quality if

$$
\frac{\partial P(g)}{\partial \lambda_{i j}}<\frac{\partial P(g)}{\partial \lambda_{u v}}, \quad \forall u v \neq i j .
$$

Definition 5.2 Under assumptions (1)-(7), transition probability $\mu_{i j}$ is the positive bottleneck transition ( $p$-BN-t) with respect to quality if

$$
\frac{\partial P(g)}{\partial \mu_{i j}}>\frac{\partial P(g)}{\partial \mu_{u v}}, \quad \forall u v \neq i j .
$$

Although Definitions 5.1 and 5.2 provide a characterization of quality bottleneck transitions, it is difficult to implement on the factory floor. First, such derivatives 
are not measurable on the factory floor. Second, there may not be a closed-form formula for these derivatives. Moreover, even if we can use the differences of $P(g)$ with respect to $\lambda_{i j}$ and $\mu_{i j}$ within a step small enough to approximate such derivatives, the computation effort is intensive. Therefore, it is necessary to develop bottleneck transition indicators for n-BN-t and p-BN-t based on the available data on the factory floor.

\subsection{Implications from Simple Cases}

We begin with two simple cases, three product types in strictly sequencing policy and two product types each with batch size three in batch policy.

\subsubsection{Three product types in strictly sequencing policy}

Assume the product sequence is 1-2-3-1. Then the transition equations can be written as follows:

$$
\begin{aligned}
& P\left(g_{11}\right)=\delta_{13} P\left(g_{31}\right)+\frac{1}{3} \mu_{13}, \\
& P\left(g_{21}\right)=\delta_{21} P\left(g_{11}\right)+\frac{1}{3} \mu_{21}, \\
& P\left(g_{31}\right)=\delta_{32} P\left(g_{21}\right)+\frac{1}{3} \mu_{32} .
\end{aligned}
$$

Then, we have

Proposition 5.1 Under assumption (1)-(7), for a given sequence $s^{1}=\{1,2,3\}$ the partial derivatives of product quality with respect to transitions can be evaluated as:

$$
\frac{\partial P\left(g_{s s}\right)}{\partial \lambda_{21}}=-\frac{P\left(g_{11}\right)}{1-\delta_{21} \delta_{13} \delta_{32}}\left(1+\delta_{32}+\delta_{32} \delta_{13}\right)
$$




$$
\begin{aligned}
\frac{\partial P\left(g_{s s}\right)}{\partial \mu_{21}} & =\frac{P\left(d_{11}\right)}{1-\delta_{21} \delta_{13} \delta_{32}}\left(1+\delta_{32}+\delta_{32} \delta_{13}\right), \\
\frac{\partial P\left(g_{s s}\right)}{\partial \lambda_{32}} & =-\frac{P\left(g_{21}\right)}{1-\delta_{21} \delta_{13} \delta_{32}}\left(1+\delta_{13}+\delta_{13} \delta_{21}\right), \\
\frac{\partial P\left(g_{s s}\right)}{\partial \mu_{32}} & =\frac{P\left(d_{21}\right)}{1-\delta_{21} \delta_{13} \delta_{32}}\left(1+\delta_{13}+\delta_{13} \delta_{21}\right), \\
\frac{\partial P\left(g_{s s}\right)}{\partial \lambda_{13}} & =-\frac{P\left(g_{31}\right)}{1-\delta_{21} \delta_{13} \delta_{32}}\left(1+\delta_{21}+\delta_{21} \delta_{32}\right), \\
\frac{\partial P\left(g_{s s}\right)}{\partial \mu_{13}} & =\frac{P\left(d_{31}\right)}{1-\delta_{21} \delta_{13} \delta_{32}}\left(1+\delta_{21}+\delta_{21} \delta_{32}\right) .
\end{aligned}
$$

Proof: See Appendix C.

Next, we consider the scenario of batch productions. A simple case of two product types and each type with batch size three is investigated first.

\subsubsection{Two product types three batch sizes in batch policy}

Assume there are two types of products, each with batch size three. The product sequence will be 1-1-1-2-2-2-1. Similar with strict sequence, the transition equations with good states only can be written as follows:

$$
\begin{aligned}
& P\left(g_{11}\right)=\delta_{12} P\left(g_{23}\right)+\frac{1}{6} \mu_{12}, \\
& P\left(g_{12}\right)=\delta_{11} P\left(g_{11}\right)+\frac{1}{6} \mu_{11}, \\
& P\left(g_{13}\right)=\delta_{11} P\left(g_{12}\right)+\frac{1}{6} \mu_{11}, \\
& P\left(g_{21}\right)=\delta_{21} P\left(g_{13}\right)+\frac{1}{6} \mu_{21}, \\
& P\left(g_{22}\right)=\delta_{22} P\left(g_{21}\right)+\frac{1}{6} \mu_{22}, \\
& P\left(g_{23}\right)=\delta_{22} P\left(g_{22}\right)+\frac{1}{6} \mu_{22} .
\end{aligned}
$$

Then, the partial derivatives of $P(g)$ with respect to its arguments $\lambda_{i j}$ and $\mu_{i j}$ can be derived. 
Proposition 5.2 Under assumption (1)-(7), for a given sequence with two product types and three batch sizes $s^{1}=\{1,1,1,2,2,2\}$, the partial derivatives of product quality with respect to transitions can be evaluated as:

$$
\begin{aligned}
\frac{\partial P\left(g_{b t}\right)}{\partial \lambda_{21}}= & -\frac{P\left(g_{13}\right)}{1-\delta_{21} \delta_{11}^{2} \delta_{12} \delta_{22}^{2}}\left(1+\delta_{22}+\delta_{22}^{2}+\delta_{22}^{2} \delta_{12}+\delta_{22}^{2} \delta_{12} \delta_{11}+\delta_{22}^{2} \delta_{12} \delta_{11}^{2}\right) \\
\frac{\partial P\left(g_{b t}\right)}{\partial \mu_{21}}= & \frac{P\left(d_{13}\right)}{1-\delta_{21} \delta_{11}^{2} \delta_{12} \delta_{22}^{2}}\left(1+\delta_{22}+\delta_{22}^{2}+\delta_{22}^{2} \delta_{12}+\delta_{22}^{2} \delta_{12} \delta_{11}+\delta_{22}^{2} \delta_{12} \delta_{11}^{2}\right) \\
\frac{\partial P\left(g_{b t}\right)}{\partial \lambda_{12}}= & -\frac{P\left(g_{13}\right)}{1-\delta_{21} \delta_{11}^{2} \delta_{12} \delta_{22}^{2}}\left(1+\delta_{11}+\delta_{11}^{2}+\delta_{11}^{2} \delta_{21}+\delta_{11}^{2} \delta_{21} \delta_{22}+\delta_{11}^{2} \delta_{21} \delta_{22}^{2}\right) \\
\frac{\partial P\left(g_{b t}\right)}{\partial \mu_{12}}= & \frac{P\left(d_{13}\right)}{1-\delta_{21} \delta_{11}^{2} \delta_{12} \delta_{22}^{2}}\left(1+\delta_{11}+\delta_{11}^{2}+\delta_{11}^{2} \delta_{21}+\delta_{11}^{2} \delta_{21} \delta_{22}+\delta_{11}^{2} \delta_{21} \delta_{22}^{2}\right) \\
\frac{\partial P\left(g_{b t}\right)}{\partial \lambda_{11}}= & -\frac{P\left(g_{12}\right)}{1-\delta_{21} \delta_{11}^{2} \delta_{12} \delta_{22}^{2}}\left(1+\delta_{21}+\delta_{21} \delta_{22}+\delta_{21} \delta_{22}^{2}+\delta_{21} \delta_{22}^{2} \delta_{12}+\delta_{21} \delta_{22}^{2} \delta_{12} \delta_{11}\right) \\
& -\frac{P\left(g_{11}\right)}{1-\delta_{21} \delta_{11}^{2} \delta_{12} \delta_{22}^{2}}\left(1+\delta_{11}+\delta_{11} \delta_{21}+\delta_{11} \delta_{21} \delta_{22}+\delta_{11} \delta_{21} \delta_{22}^{2}+\delta_{11} \delta_{21} \delta_{22}^{2} \delta_{12}\right)
\end{aligned}
$$

$$
\begin{aligned}
\frac{\partial P\left(g_{b t}\right)}{\partial \mu_{11}}= & \frac{P\left(d_{12}\right)}{1-\delta_{21} \delta_{11}^{2} \delta_{12} \delta_{22}^{2}}\left(1+\delta_{21}+\delta_{21} \delta_{22}+\delta_{21} \delta_{22}^{2}+\delta_{21} \delta_{22}^{2} \delta_{12}+\delta_{21} \delta_{22}^{2} \delta_{12} \delta_{11}\right) \\
& +\frac{P\left(d_{11}\right)}{1-\delta_{21} \delta_{11}^{2} \delta_{12} \delta_{22}^{2}}\left(1+\delta_{11}+\delta_{11} \delta_{21}+\delta_{11} \delta_{21} \delta_{22}+\delta_{11} \delta_{21} \delta_{22}^{2}+\delta_{11} \delta_{21} \delta_{22}^{2} \delta_{12}\right)
\end{aligned}
$$

$$
\begin{aligned}
\frac{\partial P\left(g_{b t}\right)}{\partial \lambda_{22}}= & -\frac{P\left(g_{22}\right)}{1-\delta_{21} \delta_{11}^{2} \delta_{12} \delta_{22}^{2}}\left(1+\delta_{12}+\delta_{12} \delta_{11}+\delta_{12} \delta_{11}^{2}+\delta_{12} \delta_{11}^{2} \delta_{21}+\delta_{12} \delta_{11}^{2} \delta_{21} \delta_{22}\right) \\
& -\frac{P\left(g_{21}\right)}{1-\delta_{21} \delta_{11}^{2} \delta_{12} \delta_{22}^{2}}\left(1+\delta_{22}+\delta_{22} \delta_{12}+\delta_{22} \delta_{12} \delta_{11}+\delta_{22} \delta_{12} \delta_{11}^{2}+\delta_{22} \delta_{12} \delta_{11}^{2} \delta_{21}\right)
\end{aligned}
$$

$$
\begin{aligned}
\frac{\partial P\left(g_{b t}\right)}{\partial \mu_{22}}= & \frac{P\left(d_{22}\right)}{1-\delta_{21} \delta_{11}^{2} \delta_{12} \delta_{22}^{2}}\left(1+\delta_{12}+\delta_{12} \delta_{11}+\delta_{12} \delta_{11}^{2}+\delta_{12} \delta_{11}^{2} \delta_{21}+\delta_{12} \delta_{11}^{2} \delta_{21} \delta_{22}\right) \\
& +\frac{P\left(d_{21}\right)}{1-\delta_{21} \delta_{11}^{2} \delta_{12} \delta_{22}^{2}}\left(1+\delta_{22}+\delta_{22} \delta_{12}+\delta_{22} \delta_{12} \delta_{11}+\delta_{22} \delta_{12} \delta_{11}^{2}+\delta_{22} \delta_{12} \delta_{11}^{2} \delta_{21}\right)
\end{aligned}
$$

Proof: See Appendix C.

As shown in Propositions 5.1 and 5.2, the partial derivatives of $P(g)$ with respect 
to $\lambda_{i j}$ and $\mu_{i j}$ are mainly dependent on probabilities in states $g_{i j}$ and $d_{i j}$ and such probabilities are not available nor collectable on the factory floor. Therefore, we seek to develop some simple approximations of these partial derivatives without knowing these probabilities, but based on the measured data only, so that it is easy to calculate and understand.

\subsection{Approximation of Derivatives of $P(g)$}

In order to seek bottleneck transition indicators, the partial derivatives of product quality with respect to transitions are expanded.

For strictly sequencing policy, we have

Proposition 5.3 Under assumption (1)-(7) and the Bernoulli-relax assumption (4.53), for a given sequence $s^{l}$, derivatives of the product quality with respect to transitions in strictly sequencing policy can be evaluated as:

$$
\begin{aligned}
& \frac{\partial P\left(g_{s s}^{l}\right)}{\partial \lambda_{s_{2}^{l}, s_{1}^{l}}}=-\frac{1}{n}\left[\left(1+\delta_{s_{3}^{l}, s_{2}^{l}}\right) e_{s_{1}^{l}, s_{n}^{l}}+\delta_{s_{1}^{l}, s_{n}^{l}}\left(e_{s_{n}^{l}, s_{n-1}^{l}}-e_{s_{1}^{l}, s_{n}^{l}}\right)\right]+o\left(\delta_{\max }^{2}\right), \\
& \frac{\partial P\left(g_{s s}^{l}\right)}{\partial \lambda_{s_{3}^{l}, s_{2}^{l}}}=-\frac{1}{n}\left[\left(1+\delta_{s_{4}^{l}, s_{3}^{l}}\right) e_{s_{2}^{l}, s_{1}^{l}}+\delta_{s_{2}^{l}, s_{1}^{l}}\left(e_{s_{1}^{l}, s_{n}^{l}}-e_{s_{2}^{l}, s_{1}^{l}}\right)\right]+o\left(\delta_{\max }^{2}\right), \\
& \frac{\partial P\left(g_{s s}^{l}\right)}{\partial \lambda_{s_{i+1}^{l}, s_{i}^{l}}}=-\frac{1}{n}\left[\left(1+\delta_{s_{i+2}^{l}, s_{i+1}^{l}}\right) e_{s_{i}^{l}, s_{i-1}^{l}}+\delta_{s_{i}^{l}, s_{i-1}^{l}}\left(e_{s_{i-1}^{l}, s_{i-2}^{l}}-e_{s_{i}^{l}, s_{i-1}^{l}}\right)\right]+o\left(\delta_{\max }^{2}\right) \text {, } \\
& i=3, \ldots, n-2, \\
& \frac{\partial P\left(g_{s s}^{l}\right)}{\partial \lambda_{s_{n}^{l}, s_{n-1}^{l}}}=-\frac{1}{n}\left[\left(1+\delta_{s_{1}^{l}, s_{n}^{l}}\right) e_{s_{n-1}^{l}, s_{n-2}^{l}}+\delta_{s_{n-1}^{l}, s_{n-2}^{l}}\left(e_{s_{n-2}^{l}, s_{n-3}^{l}}-e_{s_{n-1}^{l}, s_{n-2}^{l}}\right)\right]+o\left(\delta_{\max }^{2}\right) \text {, } \\
& \frac{\partial P\left(g_{s s}^{l}\right)}{\partial \lambda_{s_{1}^{l}, s_{n}^{l}}}=-\frac{1}{n}\left[\left(1+\delta_{s_{2}^{l}, s_{1}^{l}}\right) e_{s_{n}^{l}, s_{n-1}^{l}}+\delta_{s_{n}^{l}, s_{n-1}^{l}}\left(e_{s_{n-1}^{l}, s_{n-2}^{l}}-e_{s_{n}^{l}, s_{n-1}^{l}}\right)\right]+o\left(\delta_{\max }^{2}\right),
\end{aligned}
$$


and

$$
\begin{aligned}
\frac{\partial P\left(g_{s s}^{l}\right)}{\partial \mu_{s_{2}^{l}, s_{1}^{l}}}= & -\frac{1}{n}\left[\left(1+\delta_{s_{3}^{l}, s_{2}^{l}}\right)\left(e_{s_{1}^{l}, s_{n}^{l}}-1\right)+\delta_{s_{1}^{l}, s_{n}^{l}}\left(e_{s_{n}^{l}, s_{n-1}^{l}}-e_{s_{1}^{l}, s_{n}^{l}}\right)\right]+o\left(\delta_{\max }^{2}\right), \quad(5.22) \\
\frac{\partial P\left(g_{s s}^{l}\right)}{\partial \mu_{s_{3}, s_{2}^{l}}}= & -\frac{1}{n}\left[\left(1+\delta_{s_{4}^{l}, s_{3}^{l}}\right)\left(e_{s_{2}^{l}, s_{1}^{l}}-1\right)+\delta_{s_{2}^{l}, s_{1}^{l}}\left(e_{s_{1}^{l}, s_{n}^{l}}-e_{s_{2}^{l}, s_{1}^{l}}\right)\right]+o\left(\delta_{\max }^{2}\right), \quad i=3, \ldots, n-2, \\
\frac{\partial P\left(g_{s s}^{l}\right)}{\partial \mu_{s_{i+1}^{l}, s_{i}^{l}}}= & -\frac{1}{n}\left[\left(1+\delta_{s_{i+2}^{l}, s_{i+1}^{l}}\right)\left(e_{s_{i}^{l}, s_{i-1}^{l}}-1\right)+\delta_{s_{i}^{l}, s_{i-1}^{l}}\left(e_{s_{i-1}^{l}, s_{i-2}^{l}}-e_{s_{i}^{l}, s_{i-1}^{l}}\right)\right]+o\left(\delta_{\max }^{2}\right), \\
\frac{\partial P\left(g_{s s}^{l}\right)}{\partial \mu_{s_{n}^{l}, s_{n-1}^{l}}}= & -\frac{1}{n}\left[\left(1+\delta_{s_{1}^{l}, s_{n}^{l}}\right)\left(e_{s_{n-1}^{l}, s_{n-2}^{l}}-1\right)+\delta_{s_{n-1}^{l}, s_{n-2}^{l}}\left(e_{s_{n-2}^{l}, s_{n-3}^{l}}-e_{s_{n-1}^{l}, s_{n-2}^{l}}\right)\right] \\
& +o\left(\delta_{\max }^{2}\right), \\
\frac{\partial P\left(g_{s s}^{l}\right)}{\partial \mu_{s_{1}^{l}, s_{n}^{l}}}= & -\frac{1}{n}\left[\left(1+\delta_{s_{2}^{l}, s_{1}^{l}}\right)\left(e_{s_{n}^{l}, s_{n-1}^{l}}-1\right)+\delta_{s_{n}^{l}, s_{n-1}^{l}}\left(e_{s_{n-1}^{l}, s_{n-2}^{l}}-e_{s_{n}^{l}, s_{n-1}^{l}}\right)\right]+o\left(\delta_{\max }^{2}\right) .
\end{aligned}
$$

Proof: See Appendix C.

For batch policy, the following is obtained

Proposition 5.4 Under assumption (1)-(4) and the Bernoulli-relax assumption 4.53), for a given sequence $s^{l}$, derivatives of the product quality with respect to transitions in batch policy can be evaluated as:

when batch size $k_{s_{i}^{l}}=2$,

$$
\begin{gathered}
\frac{\partial P\left(g_{b t}^{l}\right)}{\partial \lambda_{s_{2}^{l}, s_{1}^{l}}}=-\frac{1}{K}\left[\left(1+\delta_{s_{2}^{l}, s_{2}^{l}}\right) e_{s_{1}^{l}, s_{1}^{l}}+\delta_{s_{1}^{l}, s_{1}^{l}}\left(e_{s_{1}^{l}, s_{n}^{l}}-e_{s_{1}^{l}, s_{1}^{l}}\right)\right]+o\left(\delta_{\text {max }}^{2}\right), \\
\frac{\partial P\left(g_{b t}^{l}\right)}{\partial \lambda_{s_{i+1}^{l}, s_{i}^{l}}}=-\frac{1}{K}\left[\left(1+\delta_{s_{i+1}^{l}, s_{i+1}^{l}}\right) e_{s_{i}^{l}, s_{i}^{l}}+\delta_{s_{i}^{l}, s_{i}^{l}}\left(e_{s_{i}^{l}, s_{i-1}^{l}}-e_{s_{i}^{l}, s_{i}^{l}}\right)\right]+o\left(\delta_{\max }^{2}\right), \\
i=2, \ldots, n-1, \\
\frac{\partial P\left(g_{b t}^{l}\right)}{\partial \lambda_{s_{1}^{l}, s_{n}^{l}}}=-\frac{1}{K}\left[\left(1+\delta_{s_{1}^{l}, s_{1}^{l}}\right) e_{s_{n}^{l}, s_{n}^{l}}+\delta_{s_{n}^{l}, s_{n}^{l}}\left(e_{s_{n}^{l}, s_{n-1}^{l}}-e_{s_{n}^{l}, s_{n}^{l}}\right)\right]+o\left(\delta_{\text {max }}^{2}\right), \\
\frac{\partial P\left(g_{b t}^{l}\right)}{\partial \mu_{s_{2}^{l}, s_{1}^{l}}}=-\frac{1}{K}\left[\left(1+\delta_{s_{2}^{l}, s_{2}^{l}}\right)\left(e_{s_{1}^{l}, s_{1}^{l}}-1\right)+\delta_{s_{1}^{l}, s_{1}^{l}}\left(e_{s_{1}^{l}, s_{n}^{l}}-e_{s_{1}^{l}, s_{1}^{l}}\right)\right]+o\left(\delta_{\text {max }}^{2}\right),
\end{gathered}
$$




$$
\begin{aligned}
& \frac{\partial P\left(g_{b t}^{l}\right)}{\partial \mu_{s_{i+1}^{l}, s_{i}^{l}}}=-\frac{1}{K}\left[\left(1+\delta_{s_{i+1}^{l}, s_{i+1}^{l}}\right)\left(e_{s_{i}^{l}, s_{i}^{l}}-1\right)+\delta_{s_{i}^{l}, s_{i}^{l}}\left(e_{s_{i}^{l}, s_{i-1}^{l}}-e_{s_{i}^{l}, s_{i}^{l}}\right)\right]+o\left(\delta_{\max }^{2}\right), \\
& i=2, \ldots, n-1 \\
& \frac{\partial P\left(g_{b t}^{l}\right)}{\partial \mu_{s_{1}^{l}, s_{n}^{l}}}=-\frac{1}{K}\left[\left(1+\delta_{s_{1}^{l}, s_{1}^{l}}\right)\left(e_{s_{n}^{l}, s_{n}^{l}}-1\right)+\delta_{s_{n}^{l}, s_{n}^{l}}\left(e_{s_{n}^{l}, s_{n-1}^{l}}-e_{s_{n}^{l}, s_{n}^{l}}\right)\right]+o\left(\delta_{\max }^{2}\right), \\
& \frac{\partial P\left(g_{b t}^{l}\right)}{\partial \lambda_{s_{1}^{l}, s_{1}^{l}}}=-\frac{1}{K}\left[\left(1+\delta_{s_{2}^{l}, s_{1}^{l}}\right) e_{s_{1}^{l}, s_{n}^{l}}+\delta_{s_{1}^{l}, s_{n}^{l}}\left(e_{s_{n}^{l}, s_{n}^{l}}-e_{s_{1}^{l}, s_{n}^{l}}\right)\right]+o\left(\delta_{\max }^{2}\right), \\
& \frac{\partial P\left(g_{b t}^{l}\right)}{\partial \lambda_{s_{i}^{l}, s_{i}^{l}}}=-\frac{1}{K}\left[\left(1+\delta_{s_{i+1}^{l}, s_{i}^{l}}\right) e_{s_{i}^{l}, s_{i-1}^{l}}+\delta_{s_{i}^{l}, s_{i-1}^{l}}\left(e_{s_{i-1}^{l}, s_{i-1}^{l}}-e_{s_{i}^{l}, s_{i-1}^{l}}\right)\right]+o\left(\delta_{\max }^{2}\right), \\
& i=2, \ldots, n-1, \\
& \frac{\partial P\left(g_{b t}^{l}\right)}{\partial \lambda_{s_{n}^{l}, s_{n}^{l}}}=-\frac{1}{K}\left[\left(1+\delta_{s_{1}^{l}, s_{n}^{l}}\right) e_{s_{n}^{l}, s_{n-1}^{l}}+\delta_{s_{n}^{l}, s_{n-1}^{l}}\left(e_{s_{n-1}^{l}, s_{n-1}^{l}}-e_{s_{n}^{l}, s_{n-1}^{l}}\right)\right]+o\left(\delta_{\max }^{2}\right), \\
& \frac{\partial P\left(g_{b t}^{l}\right)}{\partial \mu_{s_{1}^{l}, s_{1}^{l}}}=-\frac{1}{K}\left[\left(1+\delta_{s_{2}^{l}, s_{1}^{l}}\right)\left(e_{s_{1}^{l}, s_{n}^{l}}-1\right)+\delta_{s_{1}^{l}, s_{n}^{l}}\left(e_{s_{n}^{l}, s_{n}^{l}}-e_{s_{1}^{l}, s_{n}^{l}}\right)\right]+o\left(\delta_{\max }^{2}\right), \\
& \frac{\partial P\left(g_{b t}^{l}\right)}{\partial \mu_{s_{i}^{l}, s_{i}^{l}}}=-\frac{1}{K}\left[\left(1+\delta_{s_{i+1}^{l}, s_{i}^{l}}\right)\left(e_{s_{i}^{l}, s_{i-1}^{l}}-1\right)+\delta_{s_{i}^{l}, s_{i-1}^{l}}\left(e_{s_{i-1}^{l}, s_{i-1}^{l}}-e_{s_{i}^{l}, s_{i-1}^{l}}\right)\right]+o\left(\delta_{\max }^{2}\right) \text {, } \\
& i=2, \ldots, n-1 \\
& \frac{\partial P\left(g_{b t}^{l}\right)}{\partial \mu_{s_{n}^{l}, s_{n}^{l}}}=-\frac{1}{K}\left[\left(1+\delta_{s_{1}^{l}, s_{n}^{l}}\right)\left(e_{s_{n}^{l}, s_{n-1}^{l}}-1\right)+\delta_{s_{n}^{l}, s_{n-1}^{l}}\left(e_{s_{n-1}^{l}, s_{n-1}^{l}}-e_{s_{n}^{l}, s_{n-1}^{l}}\right)\right]+o\left(\delta_{\max }^{2}\right) .
\end{aligned}
$$

when batch size $k_{s_{i}^{l}}>2$,

$$
\begin{aligned}
& \frac{\partial P\left(g_{b t}^{l}\right)}{\partial \lambda_{s_{i+1}^{l}, s_{i}^{l}}=}-\frac{1}{K}\left[\left(1+\delta_{s_{i+1}^{l}, s_{i+1}^{l}}\right) e_{s_{i}^{l}, s_{i}^{l}}\right]+o\left(\delta_{\max }^{2}\right), \\
& i=1, \ldots, n-1, \\
& \frac{\partial P\left(g_{b t}^{l}\right)}{\partial \lambda_{s_{1}^{l}, s_{n}^{l}}=}-\frac{1}{K}\left[\left(1+\delta_{s_{1}^{l}, s_{1}^{l}}\right) e_{s_{n}^{l}, s_{n}^{l}}\right]+o\left(\delta_{\max }^{2}\right), \\
& \frac{\partial P\left(g_{b t}^{l}\right)}{\partial \mu_{s_{i+1}^{l}, s_{i}^{l}}=}-\frac{1}{K}\left[\left(1+\delta_{s_{i+1}^{l}, s_{i+1}^{l}}\right)\left(e_{s_{i}^{l}, s_{i}^{l}}-1\right)\right]+o\left(\delta_{\max }^{2}\right), \\
& i=1, \ldots, n-1,
\end{aligned}
$$




$$
\begin{aligned}
& \frac{\partial P\left(g_{b t}^{l}\right)}{\partial \mu_{s_{1}^{l}, s_{n}^{l}}}=-\frac{1}{K}\left[\left(1+\delta_{s_{1}^{l}, s_{1}^{l}}\right)\left(e_{s_{n}^{l}, s_{n}^{l}}-1\right)\right]+o\left(\delta_{\max }^{2}\right) \\
& \frac{\partial P\left(g_{b t}^{l}\right)}{\partial \lambda_{s_{1}^{l}, s_{1}^{l}}}=-\frac{1}{K}\left[\left(1+\delta_{s_{1}^{l}, s_{1}^{l}}\right) e_{s_{1}^{l}, s_{n}^{l}}+\left(k_{s_{1}^{l}}-3\right)\left(1+\delta_{s_{1}^{l}, s_{1}^{l}}\right) e_{s_{1}^{l}, s_{1}^{l}}+\left(1+\delta_{s_{2}^{l}, s_{1}^{l}}\right) e_{s_{1}^{l}, s_{1}^{l}}\right] \\
& -\frac{1}{K}\left[\delta_{s_{1}^{l}, s_{n}^{l}}\left(e_{s_{n}^{l}, s_{n}^{l}}-e_{s_{1}^{l}, s_{n}^{l}}\right)+\delta_{s_{1}^{l}, s_{1}^{l}}\left(e_{s_{1}^{l}, s_{n}^{l}}-e_{s_{1}^{l}, s_{1}^{l}}\right)\right]+o\left(\delta_{\max }^{2}\right), \\
& \frac{\partial P\left(g_{b t}^{l}\right)}{\partial \lambda_{s_{i}^{l}, s_{i}^{l}}}=-\frac{1}{K}\left[\left(1+\delta_{s_{i}^{l}, s_{i}^{l}}\right) e_{s_{i}^{l}, s_{i-1}^{l}}+\left(k_{s_{i}^{l}}-3\right)\left(1+\delta_{s_{i}^{l}, s_{i}^{l}}\right) e_{s_{i}^{l}, s_{i}^{l}}+\left(1+\delta_{s_{i+1}^{l}, s_{i}^{l}}\right) e_{s_{i}^{l}, s_{i}^{l}}\right] \\
& -\frac{1}{K}\left[\delta_{s_{i}^{l}, s_{i-1}^{l}}\left(e_{s_{i-1}^{l}, s_{i-1}^{l}}-e_{s_{i}^{l}, s_{i-1}^{l}}\right)+\delta_{s_{i}^{l}, s_{i}^{l}}\left(e_{s_{i}^{l}, s_{i-1}^{l}}-e_{s_{i}^{l}, s_{i}^{l}}\right)\right]+o\left(\delta_{\max }^{2}\right), \\
& i=2, \ldots, n, \\
& \frac{\partial P\left(g_{b t}^{l}\right)}{\partial \mu_{s_{1}^{l}, s_{1}^{l}}}=-\frac{1}{K}\left[\left(1+\delta_{s_{1}^{l}, s_{1}^{l}}\right)\left(e_{s_{1}^{l}, s_{n}^{l}}-1\right)+\left(k_{s_{1}^{l}}-3\right)\left(1+\delta_{s_{1}^{l}, s_{1}^{l}}\right)\left(e_{s_{1}^{l}, s_{1}^{l}}-1\right)\right. \\
& \left.+\left(1+\delta_{s_{2}^{l}, s_{1}^{l}}\right)\left(e_{s_{1}^{l}, s_{1}^{l}}-1\right)\right] \\
& -\frac{1}{K}\left[\delta_{s_{1}^{l}, s_{n}^{l}}\left(e_{s_{n}^{l}, s_{n}^{l}}-e_{s_{1}^{l}, s_{n}^{l}}\right)+\delta_{s_{1}^{l}, s_{1}^{l}}\left(e_{s_{1}^{l}, s_{n}^{l}}-e_{s_{1}^{l}, s_{1}^{l}}\right)\right]+o\left(\delta_{\max }^{2}\right), \\
& \frac{\partial P\left(g_{b t}^{l}\right)}{\partial \mu_{s_{i}^{l}, s_{i}^{l}}}=-\frac{1}{K}\left[\left(1+\delta_{s_{i}^{l}, s_{i}^{l}}\right)\left(e_{s_{i}^{l}, s_{i-1}^{l}}-1\right)+\left(k_{s_{i}^{l}}-3\right)\left(1+\delta_{s_{i}^{l}, s_{i}^{l}}\right)\left(e_{s_{i}^{l}, s_{i}^{l}}-1\right)\right. \\
& \left.+\left(1+\delta_{s_{i+1}^{l}, s_{i}^{l}}\right)\left(e_{s_{i}^{l}, s_{i}^{l}}-1\right)\right] \\
& -\frac{1}{K}\left[\delta_{s_{i}^{l}, s_{i-1}^{l}}\left(e_{s_{i-1}^{l}, s_{i-1}^{l}}-e_{s_{i}^{l}, s_{i-1}^{l}}\right)+\delta_{s_{i}^{l}, s_{i}^{l}}\left(e_{s_{i}^{l}, s_{i-1}^{l}}-e_{s_{i}^{l}, s_{i}^{l}}\right)\right]+o\left(\delta_{\max }^{2}\right), \\
& i=2, \ldots, n \text {. }
\end{aligned}
$$

Proof: See Appendix C.

Remark 5.1 By ignoring the higher order terms of $\delta_{\text {max }}^{2}$, the partial derivatives are still complicated. The transition bottleneck indicators can be chosen as many forms according to the following principles: first, the indicators must be accurate enough; second, parameters of the indicators must be easily measured or calculated from the factory floor; finally, the indicators can provide intrinsic insights into dominating factors of quality improvement due to better transitions. 


\subsection{Transition Bottleneck Indicators}

Based on the approximation formulas in the previous section, the bottleneck transition identification method is introduced for strictly sequencing and batch policies. Then numerical experiments are carried out to prove it feasible.

\subsubsection{Transition bottleneck indicators in strictly sequencing policy}

Given a strict sequence $s^{l}$, we define:

$$
\begin{aligned}
I_{s s}^{l}\left(\lambda_{s_{2}, s_{1}}\right) & =e_{s_{1}^{l}, s_{n}^{l}}\left(1+\delta_{s_{3}^{l}, l_{2}^{l}}\right), \\
I_{s s}^{l}\left(\lambda_{s_{i+1}, s_{i}}\right) & =e_{s_{i}^{l}, s_{i-1}^{l}}\left(1+\delta_{s_{i+2}^{l}, s_{i+1}^{l}}\right), \quad i=2, \ldots, n-2, \\
I_{s s}^{l}\left(\lambda_{s_{n}, s_{n-1}}\right) & =e_{s_{n-1}^{l}, s_{n-2}^{l}}\left(1+\delta_{s_{1}^{l}, s_{n}^{l}}\right), \\
I_{s s}^{l}\left(\lambda_{s_{1}, s_{n}}\right) & =e_{s_{n}^{l}, s_{n-1}^{l}}\left(1+\delta_{s_{2}^{l}, s_{1}^{l}}\right) .
\end{aligned}
$$

and

$$
\begin{aligned}
I_{s s}^{l}\left(\mu_{s_{2}, s_{1}}\right) & =\left(1-e_{s_{1}^{l}, s_{n}^{l}}\right)\left(1+\delta_{s_{3}^{l}, s_{2}^{l}}\right), \\
I_{s s}^{l}\left(\mu_{s_{i+1}, s_{i}}\right) & =\left(1-e_{s_{i}^{l}, s_{i-1}^{l}}\right)\left(1+\delta_{s_{i+2}^{l}, s_{i+1}^{l}}\right), \quad i=2, \ldots, n-2, \\
I_{s s}^{l}\left(\mu_{s_{n}, s_{n-1}}\right) & =\left(1-e_{s_{n-1}^{l}, s_{n-2}^{l}}\right)\left(1+\delta_{s_{1}^{l}, s_{n}^{l}}\right), \\
I_{s s}^{l}\left(\mu_{s_{1}, s_{n}}\right) & =\left(1-e_{s_{n}^{l}, s_{n-1}^{l}}\right)\left(1+\delta_{s_{2}^{l}, s_{1}^{l}}\right) .
\end{aligned}
$$

Then, the transition bottleneck indicators are introduced as follows:

$n$-BN-t Indicator: Under assumptions (1)-(7), the negative bottleneck transition (n-BN-t) in strictly sequencing policy for a given sequence $s^{l}$ is the one that satisfies

$$
\max _{\lambda} I_{s s}^{l}(\lambda),
$$


where

$$
\lambda \in\left\{\lambda_{s_{1}^{l}, s_{n}^{l}}, \lambda_{s_{2}^{l}, s_{1}^{l}}, \ldots, \lambda_{s_{i+1}^{l}, s_{i}^{l}}, \ldots, \lambda_{s_{n}^{l}, s_{n-1}^{l}}\right\}
$$

$p$-BN-t Indicator: Under assumptions (1)-(7), the positive bottleneck transition (p-BN-t) in strictly sequencing policy for a given sequence $s^{l}$ is the one that satisfies

$$
\max _{\mu} I_{s s}^{l}(\mu)
$$

where

$$
\mu \in\left\{\mu_{s_{1}^{l}, s_{n}^{l}}, \mu_{s_{2}^{l}, s_{1}^{l}}, \ldots, \mu_{s_{i+1}^{l}, s_{i}^{l}}, \ldots, \mu_{s_{n}^{l}, s_{n-1}^{l}}\right\} .
$$

Remark 5.2 A bottleneck transition can be determined by its immediately upstream and downstream transitions. The immediately upstream transition can be treated as an estimate of good part probability of start state of bottleneck transition under the Bernoulli-like assumption (See Equation 4.43). The immediately downstream transition implies that only the states of the following two products are considered when variations of transitions propagate through the state loop.

\subsubsection{Transition bottleneck indicators in batch policy}

Given a batch sequence $s^{l}$, we define:

$$
\begin{aligned}
I_{b t}^{l}\left(\lambda_{s_{i+1}, s_{i}}\right) & =e_{s_{i}^{l}, s_{i}^{l}}\left(1+\delta_{s_{i+1}^{l}, s_{i+1}^{l}}\right), \quad i=1, \ldots, n-1, \\
I_{b t}^{l}\left(\lambda_{s_{1}, s_{n}}\right) & =e_{s_{1}^{l}, s_{1}^{l}}\left(1+\delta_{s_{n}^{l}, s_{n}^{l}}\right), \\
I_{b t}^{l}\left(\lambda_{s_{1}, s_{1}}\right) & =\left\{\begin{array}{l}
e_{s_{1}^{l}, s_{n}^{l}}\left(1+\delta_{s_{2}^{l}, s_{1}^{l}}\right), \\
e_{s_{1}^{l}, s_{n}^{l}}\left(1+\delta_{s_{1}^{l}, s_{1}^{l}}\right)+\left(k_{s_{i}^{l}}-3\right) e_{s_{1}^{l}, s_{1}^{l}}\left(1+\delta_{s_{1}^{l}, s_{1}^{l}}\right)+e_{s_{1}^{l}, s_{1}^{l}}\left(1+\delta_{s_{2}^{l}, s_{1}^{l}}\right), \\
\text { when } k_{s_{i}^{l}}>2
\end{array}\right. \\
I_{b t}^{l}\left(\lambda_{s_{i}, s_{i}}\right)= & \begin{array}{l}
e_{s_{i}^{l}, s_{i-1}^{l}}\left(1+\delta_{s_{i+1}^{l}, s_{i}^{l}}\right), \\
e_{s_{i}^{l}, s_{i-1}^{l}}\left(1+\delta_{s_{i}^{l}, s_{i}^{l}}\right)+\left(k_{s_{i}^{l}}-3\right) e_{s_{i}^{l}, s_{i}^{l}}\left(1+\delta_{s_{i}^{l}, s_{i}^{l}}\right)+e_{s_{i}^{l}, s_{i}^{l}}\left(1+\delta_{s_{i+1}^{l}, s_{i}^{l}}\right), \\
\text { when } k_{s_{i}^{l}}>2
\end{array}
\end{aligned}
$$




$$
I_{b t}^{l}\left(\lambda_{s_{n}, s_{n}}\right)=\left\{\begin{array}{lc}
e_{s_{n}^{l}, s_{n-1}^{l}}\left(1+\delta_{s_{1}^{l}, s_{n}^{l}}\right), & \text { when } k_{s_{i}^{l}}=2 \\
e_{s_{n}^{l}, s_{n-1}^{l}}\left(1+\delta_{s_{n}^{l}, s_{n}^{l}}\right)+\left(k_{s_{i}^{l}}-3\right) e_{s_{n}^{l}, s_{n}^{l}}\left(1+\delta_{s_{n}^{l}, s_{n}^{l}}\right)+e_{s_{n}^{l}, s_{n}^{l}}\left(1+\delta_{s_{1}^{l}, s_{n}^{l}}\right), & \text { when } k_{s_{i}^{l}}>2
\end{array}\right.
$$

and

$$
\begin{aligned}
& I_{b t}^{l}\left(\mu_{s_{i+1}, s_{i}}\right)=\left(1-e_{s_{i}^{l}, s_{i}^{l}}\right)\left(1+\delta_{s_{i+1}^{l}, s_{i+1}^{l}}\right), \quad i=1, \ldots, n-1 \\
& I_{b t}^{l}\left(\mu_{s_{1}, s_{n}}\right)=\left(1-e_{s_{1}^{l}, s_{1}^{l}}\right)\left(1+\delta_{s_{n}^{l}, s_{n}^{l}}\right), \\
& I_{b t}^{l}\left(\mu_{s_{1}, s_{1}}\right)=\left\{\begin{array}{lr}
\left(1-e_{s_{1}^{l}, s_{n}^{l}}\right)\left(1+\delta_{s_{2}^{l}, s_{1}^{l}}\right), & \text { when } k_{s_{i}^{l}}=2 \\
\left(1-e_{s_{1}^{l}, s_{n}^{l}}\right)\left(1+\delta_{s_{1}^{l}, s_{1}^{l}}\right)+\left(k_{s_{i}^{l}}-3\right)\left(1-e_{s_{1}^{l}, s_{1}^{l}}\right)\left(1+\delta_{s_{1}^{l}, s_{1}^{l}}\right) & \text { when } k_{s_{i}^{l}}>2
\end{array}\right. \\
& I_{b t}^{l}\left(\mu_{s_{i}, s_{i}}\right)=\left\{\begin{array}{lr}
\left(1-e_{s_{i}^{l}, s_{i-1}^{l}}\right)\left(1+\delta_{s_{i+1}^{l}, s_{i}^{l}}\right), & \text { when } k_{s_{i}^{l}}=2 \\
\left(1-e_{s_{i}^{l}, s_{i-1}^{l}}\right)\left(1+\delta_{s_{i}^{l}, s_{i}^{l}}\right)+\left(k_{s_{i}^{l}}-3\right)\left(1-e_{s_{i}^{l}, s_{i}^{l}}\right)\left(1+\delta_{s_{i}^{l}, s_{i}^{l}}\right) & \text { when } k_{s_{i}^{l}}>2
\end{array}\right. \\
& i=2, \ldots, n-1, \\
& I_{b t}^{l}\left(\mu_{s_{n}, s_{n}}\right)=\left\{\begin{array}{lr}
\left(1-e_{s_{n}^{l}, s_{n-1}^{l}}\right)\left(1+\delta_{s_{1}^{l}, s_{n}^{l}}\right), & \text { when } k_{s_{i}^{l}}=2 \\
\left(1-e_{s_{n}^{l}, s_{n-1}^{l}}\right)\left(1+\delta_{s_{n}^{l}, s_{n}^{l}}\right)+\left(k_{s_{i}^{l}}-3\right)\left(1-e_{s_{n}^{l}, s_{n}^{l}}\right)\left(1+\delta_{s_{n}^{l}, s_{n}^{l}}\right) \\
+\left(1-e_{s_{n}^{l}, s_{n}^{l}}\right)\left(1+\delta_{s_{1}^{l}, s_{n}^{l}}\right), & \text { when } k_{s_{i}^{l}}>2
\end{array}\right.
\end{aligned}
$$

Then, the transition bottleneck indicators are introduced as follows:

$n$-BN-t Indicator: Under assumptions (1)-(7), the negative bottleneck transition (n-BN-t) in batch policy for a given sequence $s^{l}$ is the one that satisfies

$$
\max _{\lambda} I_{b t}^{l}(\lambda)
$$

where

$$
\lambda \in\left\{\lambda_{s_{1}^{l}, s_{n}^{l}}, \lambda_{s_{2}^{l}, s_{1}^{l}}, \ldots, \lambda_{s_{i+1}^{l}, s_{i}^{l}}, \ldots, \lambda_{s_{n}^{l}, s_{n-1}^{l}} ; \lambda_{s_{1}^{l}, s_{1}^{l}}, \ldots, \lambda_{s_{i}^{l}, s_{i}^{l}}, \ldots, \lambda_{s_{n}^{l}, s_{n}^{l}}\right\}
$$


p-BN-t Indicator: Under assumptions (1)-(7), the positive bottleneck transition (p-BN-t) in batch policy for a given sequence $s^{l}$ is the one that satisfies

$$
\max _{\mu} I_{b t}^{l}(\mu)
$$

where

$$
\mu \in\left\{\mu_{s_{1}^{l}, s_{n}^{l}}, \mu_{s_{2}^{l}, s_{1}^{l}}, \ldots, \mu_{s_{i+1}^{l}, s_{i}^{l}}, \ldots, \mu_{s_{n}^{l}, s_{n-1}^{l}} ; \mu_{s_{1}^{l}, s_{1}^{l}}, \ldots, \mu_{s_{i}^{l}, s_{i}^{l}}, \ldots, \mu_{s_{n}^{l}, s_{n}^{l}}\right\}
$$

\subsubsection{Numerical justification}

Numerical experiments have been carried out to justify the bottleneck indicators above. More than 10000 cases are generated and tested. For $\delta_{\max }=0.2$, we randomly and equiprobably select $\mu_{i j}$ and then $\lambda_{i j}$ such that

$$
\begin{aligned}
& \lambda_{i j} \in\left(0, \delta_{\max }\right], \\
& \mu_{i j} \in\left[1-\delta_{\max }, 1\right),
\end{aligned}
$$

Such a selection implies that

$$
1-\delta_{\max }<\lambda_{i j}+\mu_{i j}<1+\delta_{\max }
$$

In addition, batch size $k_{i}$ is also selected randomly from

$$
k_{i} \in\{2, \ldots, 6\}
$$

The n-BN-t and p-BN-t indentified through indicators are compared with the results obtained by computing and selecting the largest $\Delta P(g) / \Delta \lambda_{i j}$ and $\Delta P(g) / \Delta \mu_{i j}$ with $\Delta \lambda_{i j}=0.001$ and $\Delta \mu_{i j}=0.001$, respectively. When both methods result in same transition, a correct identification is obtained. In addition, the differences in partial 
derivatives of two bottleneck transitions from both methods are investigated when indicators lead to incorrect bottleneck transitions. Define

$$
\begin{aligned}
\Delta_{t} & =\left|\frac{\partial P\left(g^{l}\right)}{\partial \lambda_{i j}}-\frac{\partial P\left(g^{l}\right)}{\partial \lambda_{u v}}\right| \text { or }\left|\frac{\partial P\left(g^{l}\right)}{\partial \mu_{i j}}-\frac{\partial P\left(g^{l}\right)}{\partial \mu_{u v}}\right|, \\
\epsilon_{t} & =\frac{\left|\frac{\partial P\left(g^{l}\right)}{\partial \lambda_{i j}}-\frac{\partial P\left(g^{l}\right)}{\partial \lambda_{u v}}\right|}{\frac{\partial P\left(g^{l}\right)}{\partial \lambda_{i j}}} \cdot 100 \% \text { or } \frac{\left|\frac{\partial P\left(g^{l}\right)}{\partial \mu_{i j}}-\frac{\partial P\left(g^{l}\right)}{\partial \mu_{u v}}\right|}{\frac{\partial P\left(g^{l}\right)}{\partial \mu_{i j}}} \cdot 100 \% .
\end{aligned}
$$

where $\lambda_{i j}$ and $\mu_{i j}$ are the bottleneck transitions obtained based on the largest $\Delta P(g) / \Delta \lambda_{i j}$ and $\Delta P(g) / \Delta \mu_{i j}$, and $\lambda_{u v}$ and $\mu_{u v}$ are the bottleneck transitions identified by indicators.

Table 5.1: Effectiveness of $\mathrm{n}-\mathrm{BN}-\mathrm{t}$ and $\mathrm{p}-\mathrm{BN}-\mathrm{t}$ indicators in strictly sequencing pol$\operatorname{icy}(\%)$

\begin{tabular}{|c|c|c|c|c|c|}
\hline$n$ & 2 & 3 & 4 & 5 & 6 \\
\hline n-BN-t & 99.30 & 96.25 & 94.20 & 94.41 & 94.22 \\
\hline p-BN-t & 99.64 & 98.05 & 95.44 & 93.97 & 93.00 \\
\hline
\end{tabular}

Table 5.2: Errors when n-BN-t and p-BN-t indicators lead to incorrect comparisons in strictly sequencing policy

\begin{tabular}{|c|c|c|c|c|c|}
\hline$n$ & 2 & 3 & 4 & 5 & 6 \\
\hline 2nd largest slope $(\%)$ & 100 & 98.13 & 95.69 & 95.35 & 93.77 \\
\hline Average $\Delta_{t}$ & 0.0015 & 0.0024 & 0.0022 & 0.0016 & 0.0013 \\
\hline Maximal $\Delta_{t}$ & 0.0054 & 0.0113 & 0.0227 & 0.0082 & 0.0081 \\
\hline Average $\epsilon_{t}(\%)$ & 0.33 & 0.77 & 0.87 & 0.81 & 0.79 \\
\hline Maximal $\epsilon_{t}(\%)$ & 1.37 & 3.47 & 7.82 & 4.09 & 4.46 \\
\hline
\end{tabular}

(a) n-BN-t

\begin{tabular}{|c|c|c|c|c|c|}
\hline$n$ & 2 & 3 & 4 & 5 & 6 \\
\hline 2nd largest slope $(\%)$ & 100 & 100 & 98.25 & 97.68 & 95.29 \\
\hline Average $\Delta_{t}$ & $5.51 \mathrm{E}-04$ & $9.26 \mathrm{E}-04$ & 0.0015 & 0.0011 & $9.76 \mathrm{E}-04$ \\
\hline Maximal $\Delta_{t}$ & 0.0020 & 0.0052 & 0.0071 & 0.0063 & 0.0045 \\
\hline Average $\epsilon_{t}(\%)$ & 0.89 & 2.13 & 3.64 & 3.34 & 3.51 \\
\hline Maximal $\epsilon_{t}(\%)$ & 3.05 & 10.22 & 14.74 & 14.79 & 15.55 \\
\hline
\end{tabular}

(b) p-BN-t 
Table 5.3: Effectiveness of n-BN-t and p-BN-t indicators in batch policy(\%)

\begin{tabular}{|c|c|c|c|c|c|}
\hline$n$ & 2 & 3 & 4 & 5 & 6 \\
\hline n-BN-t & 99.48 & 99.41 & 99.36 & 99.21 & 98.97 \\
\hline p-BN-t & 97.81 & 97.16 & 97.36 & 97.00 & 97.06 \\
\hline
\end{tabular}

Table 5.4: Errors when n-BN-t and p-BN-t indicators lead to incorrect comparisons in batch policy

\begin{tabular}{|c|c|c|c|c|c|}
\hline$n$ & 2 & 3 & 4 & 5 & 6 \\
\hline 2nd largest slope (\%) & 100 & 98.61 & 98.44 & 98.73 & 98.06 \\
\hline Average $\Delta_{t}$ & 0.0015 & $9.98 \mathrm{E}-04$ & $7.91 \mathrm{E}-04$ & $7.58 \mathrm{E}-04$ & $5.51 \mathrm{E}-04$ \\
\hline Maximal $\Delta_{t}$ & 0.0066 & 0.0041 & 0.0032 & 0.0031 & 0.0024 \\
\hline Average $\epsilon_{t}(\%)$ & 0.56 & 0.45 & 0.43 & 0.44 & 0.36 \\
\hline Maximal $\epsilon_{t}(\%)$ & 2.32 & 2.30 & 2.38 & 1.68 & 1.63 \\
\hline
\end{tabular}

(a) n-BN-t

\begin{tabular}{|c|c|c|c|c|c|}
\hline$n$ & 2 & 3 & 4 & 5 & 6 \\
\hline 2nd largest slope (\%) & 99.09 & 98.94 & 97.45 & 97.67 & 96.71 \\
\hline Average $\Delta_{t}$ & 0.0014 & $9.36 \mathrm{E}-04$ & $6.74 \mathrm{E}-04$ & $5.35 \mathrm{E}-04$ & $4.10 \mathrm{E}-04$ \\
\hline Maximal $\Delta_{t}$ & 0.0059 & 0.0056 & 0.0035 & 0.0037 & 0.0034 \\
\hline Average $\epsilon_{t}(\%)$ & 4.11 & 3.83 & 3.27 & 2.99 & 2.48 \\
\hline Maximal $\epsilon_{t}(\%)$ & 20.21 & 22.92 & 17.99 & 17.87 & 20.61 \\
\hline
\end{tabular}

(b) p-BN-t 
It is shown in Tables 5.1 and 5.3 that the bottleneck indicators have resulted in high accuracy to identify the bottlenecks, with more than $93 \%$ correctness in strictly sequencing policy and $97 \%$ in batch policy. The differences in partial derivatives of two bottleneck transitions from both methods shown in Tables 5.2 and 5.4 are very small when incorrect bottleneck transitions are identified using indicators. In this case, the transitions identified by indicators can be treated as real bottleneck transitions. Note that maximal $\epsilon_{t}$ is usually large. That is because the partial derivatives of bottleneck transitions in that cases are not large compared with the maximal $\Delta_{t}$ which is already small. It implies that in some cases quality improvement due to better transitions can not be expected significant. Therefore, we conclude that these bottleneck indicators can be used for identifying the bottleneck transitions for quality improvement.

\subsection{Summary}

Identifying the bottleneck is an effective way to improve the quality. In this chapter, we define a quality bottleneck transition as the transition that impedes quality performance in the strongest manner. A method to evaluate the sensitivity of quality performance with respect to its transition probabilities is presented and quality bottleneck transition indicators based on the data collected on the factory floor are proposed. Using these indicators, negative and positive quality bottleneck transitions can be identified effectively. Such methods provide a quantitative and practical tool for production engineers and managers to improve quality in flexible manufacturing systems. 


\section{CHAPTER 6}

\section{PRODUCT SEQUENCING TO REDUCE ENERGY CONSUMPTION AND ATMOSPHERIC EMISSIONS}

This chapter is to apply optimal batch and scheduling method to reduce energy consumption and atmospheric emissions in automotive paint shops. Specifically, by using the quality evaluation models, we analyze the energy usage and emission levels of a vehicle painting process. The benefits in energy savings through optimal batch (to group the vehicles with the same color together) and scheduling policies (to arrange color sequence) are investigated.

\subsection{Job Flow, Energy and Emission Evaluation in Paint Shops 6.1.1 Job flow}

Automotive painting is an extremely complex process. In order to improve paint quality, every vehicle will be inspected after color coating and baking operations. Vehicles with paint defects will be either repaired or repainted. A typical structure of painting process with repair is illustrated in Figure 6.1.

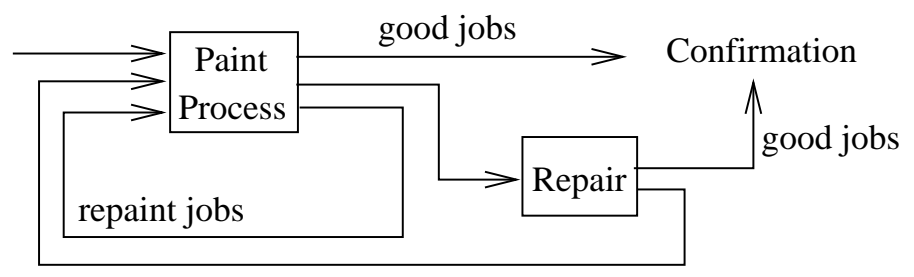

Figure 6.1: Illustration of job flow in paint in automotive assembly plant 
In such system, jobs after painting processes are inspected. A job is sent to the confirmation station if it has good paint quality. The ratio that the jobs pass the inspection after first time processing is referred to as first time quality (FTQ). All defective jobs are routed to either repair or repaint based on the nature and severity of the defects. Jobs with severe defects are sent back to the painting booths for repaint, i.e., the complete painting process will be repeated. In the repair process, either new parts (e.g., panels) are exchanged or light defects are fixed (e.g., scratches are polished). After repair, jobs are inspected again. Jobs now with good quality are routed to confirmation station. However, there is a possibility that some jobs still have bad quality so that they will be sent back to painting both for repaint. In most paint shops, the first time quality is typically above $80 \%$, but the repaint quality is much lower than the FTQ. The quality ratio is decreasing rapidly with more repaints. In practice, a vehicle may be repainted no more than three times.

To analyze the energy consumption of the painting process, we simplify the process in Figure 6.1 by ignoring the energy costs in repairing process, since such process is typically finished manually. Then we aggregate the good job ratios in painting booths and repair shops to define the quality buy rate (QBR) of the overall painting process, and obtain a simplified model as shown in Figure 6.2. In other words, the good jobs leaving from the painting process include the jobs pass the inspection directly from painting booths and those go to confirmation station from repair. Similarly, the repaint jobs are comprised by jobs with severe defects which are sent directly for repaint after painting booths and inspection, and jobs which could not meet quality standard even after repair. Using such a simplified model, we evaluate the energy 
consumption and Carbon emissions in painting process next.

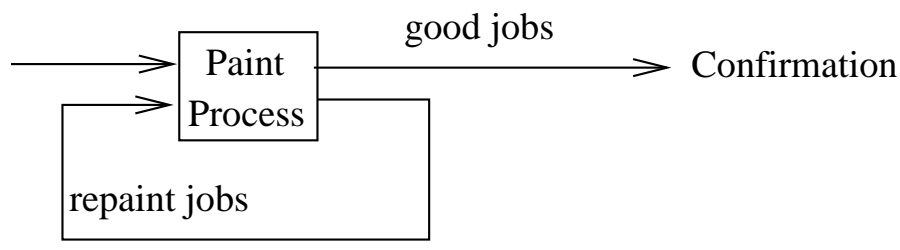

Figure 6.2: Simplified painting process

\subsubsection{Energy evaluation}

Introduce the following notation:

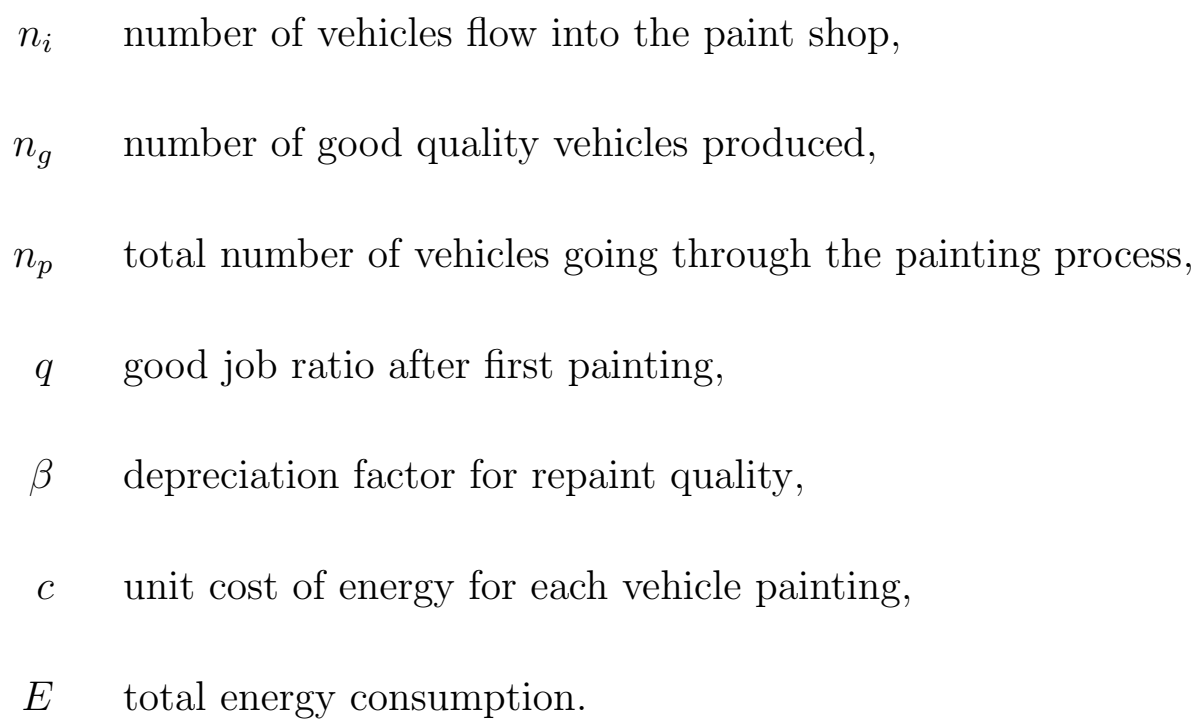

Note that the number of vehicles flow into the paint shop per day, $n_{i}$, is more than the number of vehicles arriving the confirmation station, $n_{g}$, due to possible scraps (i.e., vehicles could not obtain good quality after three repaints will be scraped). Then, the following relationship is obtained:

$n_{g}=n_{i} q+n_{i}(1-q) \beta q+n_{i}(1-q)(1-\beta q) \beta^{2} q+n_{i}(1-q)(1-\beta q)\left(1-\beta^{2} q\right) \beta^{3} q \cdot(6.1)$

The necessary number of vehicles $n_{i}$ to achieve production volume $n_{g}$ can be calculated by 


$$
n_{i}=\frac{n_{g}}{q\left[1+(1-q) \beta+(1-q)(1-\beta q) \beta^{2}+(1-q)(1-\beta q)\left(1-\beta^{2} q\right) \beta^{3}\right]} .
$$

Then, the total number of vehicles going through the painting booths and ovens is

$$
n_{p}=n_{i}+n_{i}(1-q)+n_{i}(1-q)(1-\beta q)+n_{i}(1-q)(1-\beta q)\left(1-\beta^{2} q\right) .
$$

Finally, the energy consumption $E$ is described as:

$$
E=c n_{p}=\frac{c n_{g}\left[1+(1-q)+(1-q)(1-\beta q)+(1-q)(1-\beta q)\left(1-\beta^{2} q\right)\right]}{q\left[1+(1-q) \beta+(1-q)(1-\beta q) \beta^{2}+(1-q)(1-\beta q)\left(1-\beta^{2} q\right) \beta^{3}\right]} .
$$

Consider a painting process having two scheduling procedures, whose first time quality performances are characterized as $q_{1}$ and $q_{2}$. Assuming all other parameters, $\beta, c$, and $n_{g}$ are the same. Then the energy consumptions of these two procedures, $E_{1}$ and $E_{2}$, can be compared as

$$
\begin{aligned}
\frac{E_{1}}{E_{2}=} & \frac{\frac{c n_{g}\left[1+\left(1-q_{1}\right)+\left(1-q_{1}\right)\left(1-\beta q_{1}\right)+\left(1-q_{1}\right)\left(1-\beta q_{1}\right)\left(1-\beta^{2} q_{1}\right)\right]}{q_{1}\left[1+\left(1-q_{1}\right) \beta+\left(1-q_{1}\right)\left(1-\beta q_{1}\right) \beta^{2}+\left(1-q_{1}\right)\left(1-\beta q_{1}\right)\left(1-\beta^{2} q_{1}\right) \beta^{3}\right]}}{\frac{c n_{g}\left[1+\left(1-q_{2}\right)+\left(1-q_{2}\right)\left(1-\beta q_{2}\right)+\left(1-q_{2}\right)\left(1-\beta q_{2}\right)\left(1-\beta^{2} q_{2}\right)\right]}{q_{2}\left[1+\left(1-q_{2}\right) \beta+\left(1-q_{2}\right)\left(1-\beta q_{2}\right) \beta^{2}+\left(1-q_{2}\right)\left(1-\beta q_{2}\right)\left(1-\beta^{2} q_{2}\right) \beta^{3}\right]}} \\
= & \frac{\left.1+\left(1-q_{1}\right)+\left(1-q_{1}\right)\left(1-\beta q_{1}\right)+\left(1-q_{1}\right)\left(1-\beta q_{1}\right)\left(1-\beta^{2} q_{1}\right)\right]}{1+\left(1-q_{2}\right)+\left(1-q_{2}\right)\left(1-\beta q_{2}\right)+\left(1-q_{2}\right)\left(1-\beta q_{2}\right)\left(1-\beta^{2} q_{2}\right)} \\
& \cdot \frac{1+\left(1-q_{2}\right) \beta+\left(1-q_{2}\right)\left(1-\beta q_{2}\right) \beta^{2}+\left(1-q_{2}\right)\left(1-\beta q_{2}\right)\left(1-\beta^{2} q_{2}\right) \beta^{3}}{1+\left(1-q_{1}\right) \beta+\left(1-q_{1}\right)\left(1-\beta q_{1}\right) \beta^{2}+\left(1-q_{1}\right)\left(1-\beta q_{1}\right)\left(1-\beta^{2} q_{1}\right) \beta^{3}} \cdot \frac{q_{2}}{q_{1}}(6.5)
\end{aligned}
$$

Clearly, to evaluate the energy consumption $E$ or to compare $E$ s under different policies (i.e., to evaluate $E_{1} / E_{2}$ ), the good job ratio $q$ needs to be known first. In order to investigate the impacts of batch and sequencing policies on energy consumption, a quality evaluation model for paint system with batch production is needed. Such a model is described in Chapter 3. 


\subsubsection{Emission evaluation}

Introduce the following notation:

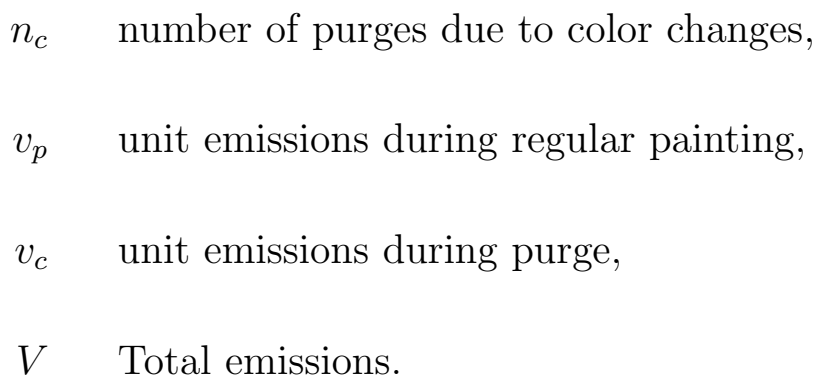

Other notation, $n_{g}, n_{i}, n_{p}$, and $\beta$ are the same as in the energy model.

The total number of vehicles going through the painting process is $n_{p}$, thus, the emissions during regular painting are $n_{p} v_{p}$. In addition, the purge processes generate emissions of $n_{c} v_{c}$. Therefore, the overall painting procedures have emissions of

$$
V=n_{p} v_{p}+n_{c} v_{c}=v_{p}\left(n_{p}+\alpha n_{c}\right)
$$

where $n_{p}$ is calculated in $(6.3), \alpha$ is the ration of emissions generated during purge and regular painting, i.e., $\alpha=v_{c} / v_{p}$, and $n_{c}$ is dependent on the batch and scheduling policy, which will be introduced in Section 6.2.

Again, with the knowledge of paint quality, the total emissions $V$ can be calculated or the comparison of $V$ s can be carried out.

\subsection{Evaluation of Energy Usage and Atmospheric Emission in Paint Shops}

\subsubsection{Comparison of energy usage}

To evaluate the energy consumption in paint shops, we compare painting processes under different scheduling policies by randomly selecting the quality parameters. Specifically, the quality failure probabilities and efficiencies, and batch size are randomly 
and uniformly selected from the following sets:

$$
\begin{aligned}
\lambda_{i i} & \in[0.05,0.15], \\
\lambda_{i j} & \in[0.1,0.2], \\
e_{i i} & \in[0.88,0.96], \\
e_{i j} & \in[0.85,0.93], \\
k & \in\{2,3,4,5,6\},
\end{aligned}
$$

and $\mu_{i i}$ is calculated from $\lambda_{i i}$ and $e_{i i}$. Similar calculation is used for $\mu_{i j}$. In addition, since in practice, the quality efficiency without color switch is typically higher than that with color change, i.e.,

$$
e_{i i}>e_{i j}, \quad j \neq i
$$

thus, only the cases that $e_{i i}>e_{i j}$ are selected. The batch sizes for all colors are assumed equal since we would like to compare with the scenario of strictly sequencing policy. The depreciation factor of repaint quality, $\beta$, is chosen as $1,0.75$ and 0.5 .

A total of 10,000 systems are generated and compared for energy consumption under optimal and worst batch policies, optimal and worst strictly sequencing policies, and randomly mixed policy (more details in paper [21]). The energy consumptions under these policies are denoted as $E_{b t_{-} o p}$ (batch policy with optimal sequence in terms of quality), $E_{b t_{-} w t}$ (batch policy with worst sequence), $E_{s s_{-} o p}$ (strictly sequencing policy with optimal sequence), $E_{s s_{-} w t}$ (strictly sequencing policy with optimal sequence) and $E_{r n d}$ (sequence in randomly mixed policy), respectively. The results of these comparisons are shown in Figures 6.36.6. 


\section{Batch policy with optimal quality vs. strictly sequencing policy with worst quality}

First we compare the results or optimal batch policy with strictly sequencing policy having worst quality $\left(E_{b t \_o p} / E_{s s \_w t}\right)$. Three scenarios with different depreciation factors in repaint quality, $\beta=1,0.75$ and 0.5 , are considered in Figure 6.3, where the average reductions of energy are illustrated. It is shown that when there is no quality depreciation in repaint, at average about 4-5\% savings in energy can be obtained. When repaint quality is becoming lower (which is typical in most paint shops), the savings are significantly higher, up to $11 \%$ when $\beta=0.5$.

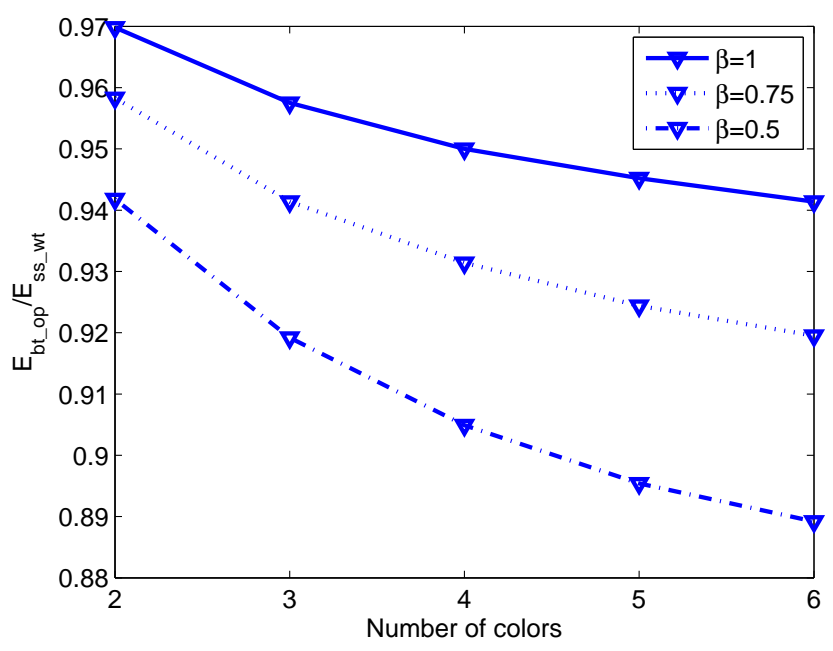

Figure 6.3: Energy usage of optimal sequence in batch policy vs worst sequence in strictly sequencing policy

\section{Batch policy with optimal quality vs. strictly sequencing policy with op- timal quality}

The above comparison illustrate the best case we may have. Next we compare batch policy with strictly sequencing policy, both under optimal sequence schedule $\left(E_{b t \_o p} / E_{s s_{-} o p}\right)$. Such a comparison can justify the advantage using batch in paint 
shops. The results are similar but with smaller magnitude, as shown in Figure 6.4. When $\beta=1$, there is about $3 \%$ savings on average. Such savings become larger when depreciation factor $\beta$ is smaller. If $\beta=0.5$, such savings are almost $5-6 \%$, which again is significant.

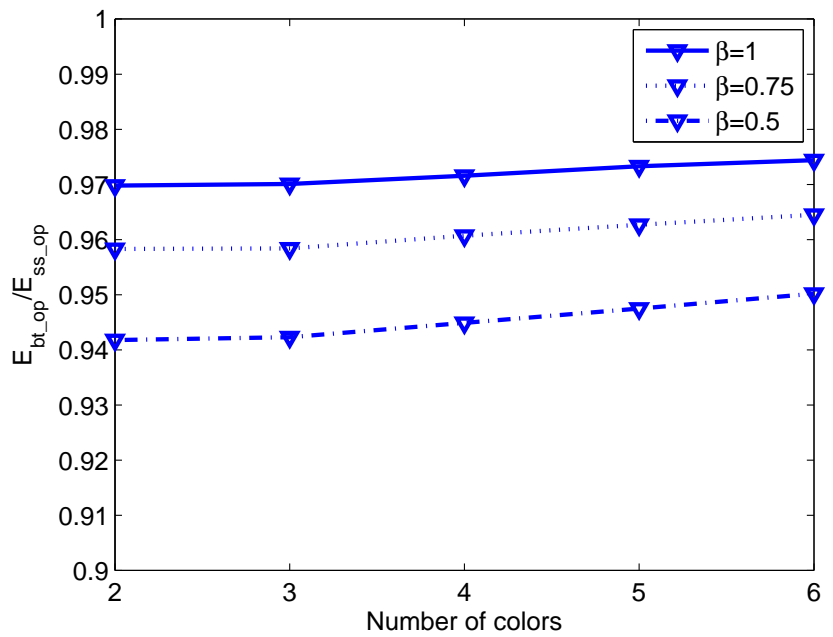

Figure 6.4: Energy usage of optimal sequence in batch policy vs optimal sequence in strictly sequencing policy

\section{Batch policy with optimal quality vs. batch policy with worst quality}

The above results show that batch policy can lead to substantial savings in energy consumption. Next we compare the impact of sequencing in both batch production environment, i.e., compare optimal and worst sequences with respect to quality in batch production $\left(E_{b t_{-o p}} / E_{b t_{-} w t}\right)$. We observe that we can have close to $1 \%$ reduction in energy when no repaint quality depreciation is assumed (see Figure 6.5). When there is depreciation during repaint, the average savings is up to $2 \%$. These results indicate that selecting the optimal batch and sequence schedule is important to achieve maximal improvement in energy efficiency. 


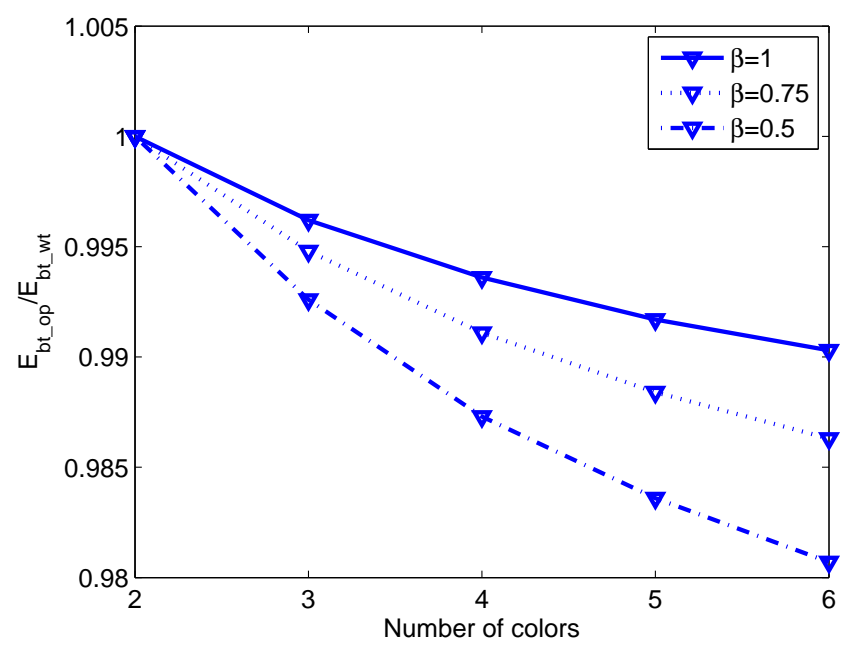

Figure 6.5: Energy usage of optimal sequence vs worst sequence in batch policy

\section{Batch policy with optimal quality vs. randomly mixed policy with average quality}

Finally, since some paint shops are operating similar to randomly mixed scenario, we compare it with optimal batch and sequence schedule. Here, randomly generated incoming vehicle sequences are evaluated and average quality is calculated for comparison. Figure 6.6 illustrates the comparison results between $E_{b t_{-} o p}$ and $E_{r n d}$. As one can see, the energy savings that can be obtained are from $2-3 \%$ to $5-6 \%$ for $\beta$ s from 1 to 0.5 .

In summary, these results suggest that introducing optimal batch and sequencing policies can improve the quality performance and reduce the energy consumption. Since a huge amount of energy is consumed in automotive paint shops, in particular in painting process areas, even 2 or $3 \%$ reduction will be significant. 


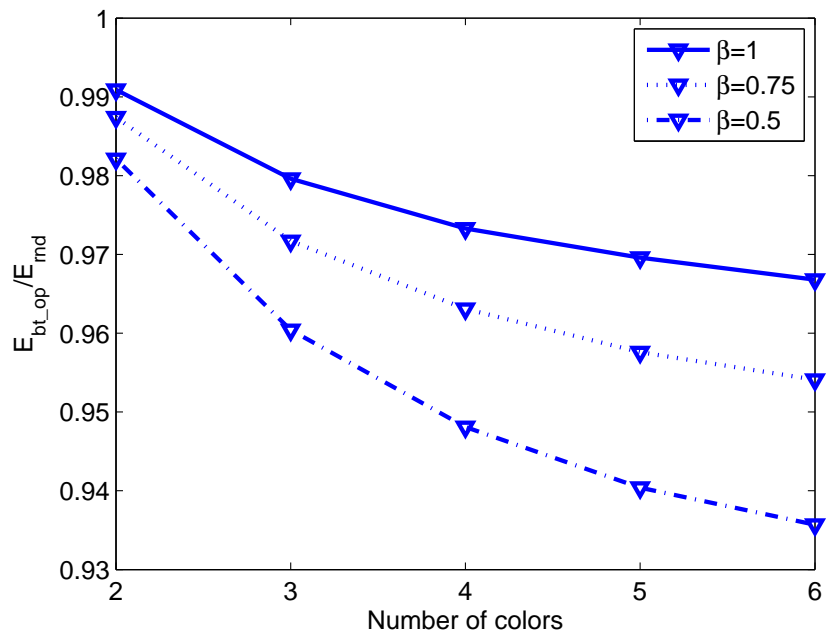

Figure 6.6: Energy usage of optimal sequence in batch policy vs randomly mixed policy

\subsubsection{Emission Comparison}

First, to compare the emissions under different batch and sequencing policies, the number of color changes during the whole painting procedure for $n_{p}$ vehicles need to be estimated.

- For strictly sequencing policy, the number of color changes equals to $n_{p}-1$.

- For batch policy with batch size $k$, we have $\left\lceil\frac{n_{i}}{k}-1\right\rceil$ color changes for first time paint vehicles, $\left\lceil\frac{n_{i}(1-q)}{k}-1\right\rceil$ for first repaint vehicles, $\left\lceil\frac{n_{i}(1-q)(1-\beta q)}{k}-1\right\rceil$ and $\left\lceil\frac{n_{i}(1-q)(1-\beta q)\left(1-\beta^{2} q\right)}{k}-1\right\rceil$ for second and third time repaint ones, respectively.

- If it is under randomly mixed policy, the average number of color changes for first time paint vehicles will be $\left\lceil\frac{\left(n_{i}-1\right)(n-1)}{n}\right\rceil$. Then the numbers of purges for first, second, and third time repaint vehicles are, $\left\lceil\frac{\left(n_{i}-1\right)(n-1)}{n}(1-q)\right\rceil,\left\lceil\frac{\left(n_{i}-1\right)(n-1)}{n}(1-\right.$ $q)(1-\beta q)\rceil$ and $\left\lceil\frac{\left(n_{i}-1\right)(n-1)}{n}(1-q)(1-\beta q)\left(1-\beta^{2} q\right)\right\rceil$, respectively. 
- Therefore, the total number of purges will be

strictly sequencing policy

$$
n_{c}=n_{p}-1,
$$

batch policy

$$
\begin{aligned}
n_{c}= & \left\lceil\frac{n_{i}}{k}-1\right\rceil+\left\lceil\frac{n_{i}(1-q)}{k}-1\right\rceil+\left\lceil\frac{n_{i}(1-q)(1-\beta q)}{k}-1\right\rceil \\
& +\left\lceil\frac{n_{i}(1-q)(1-\beta q)\left(1-\beta^{2} q\right)}{k}-1\right\rceil,
\end{aligned}
$$

randomly mixed policy

$$
\begin{aligned}
n_{c}= & \left\lceil\frac{\left(n_{i}-1\right)(n-1)}{n}\right\rceil+\left\lceil\frac{\left(n_{i}-1\right)(n-1)}{n}(1-q)\right\rceil \\
& +\left\lceil\frac{\left(n_{i}-1\right)(n-1)}{n}(1-q)(1-\beta q)\right\rceil \\
& +\left\lceil\frac{\left(n_{i}-1\right)(n-1)}{n}(1-q)(1-\beta q)\left(1-\beta^{2} q\right)\right\rceil .
\end{aligned}
$$

Denote the number of vehicles going through the painting process and number of color changes for scheduling procedure $j$ (batch, strictly sequencing, and randomly mixed) as $n_{p, j}$ and $n_{c, j}$, respectively. Then the the total emissions in two scheduling procedures, $V_{1}$ and $V_{2}$, can be compared as

$$
\frac{V_{1}}{V_{2}}=\frac{v_{p} n_{p, 1}+n_{c, 1} v_{c}}{v_{p} n_{p, 2}+n_{c, 2} v_{c}},
$$

where $n_{p, j}$ is calculated using 6.3$)$.

Again a total of 10,000 systems are generated and compared for energy consumption under optimal and worst batch policies, optimal and worst strictly sequencing policies, and randomly mixed policy. The resulting Carbon emissions under these policies are denoted as $V_{b t \_o p}$ (batch policy with optimal sequence in terms of quality), $V_{b t \_w t}$ (batch policy with worst sequence), $V_{s s_{-} o p}$ (strictly sequencing policy with 
optimal sequence), $V_{s s_{-} w t}$ (strictly sequencing policy with optimal sequence) and $V_{\text {rnd }}$ (sequence in randomly mixed policy), respectively. The results of these comparisons are similar to those in energy models, and are illustrated in Figures 6.3 6.6.

\section{Batch policy with optimal quality vs. strictly sequencing policy with worst quality}

First we compare the results of emissions under optimal batch policy with strictly sequencing policy, both having worst quality $\left(V_{b t_{-} o p} / V_{s s_{-} w t}\right)$. Again, we consider three scenarios of depreciation factors in repaint quality, $\beta=1,0.75$ and 0.5 . Figure 6.7 illustrates the average reductions of emissions. If there is no depreciation in quality during repaints, at average about $7-8 \%$ reduction of emissions can be achieved. When $\beta$ is less than 1, i.e., repaint quality is becoming lower, the emission reductions are more significant, up to 11 and $14 \%$, when $\beta=0.75$ and 0.5 , respectively.

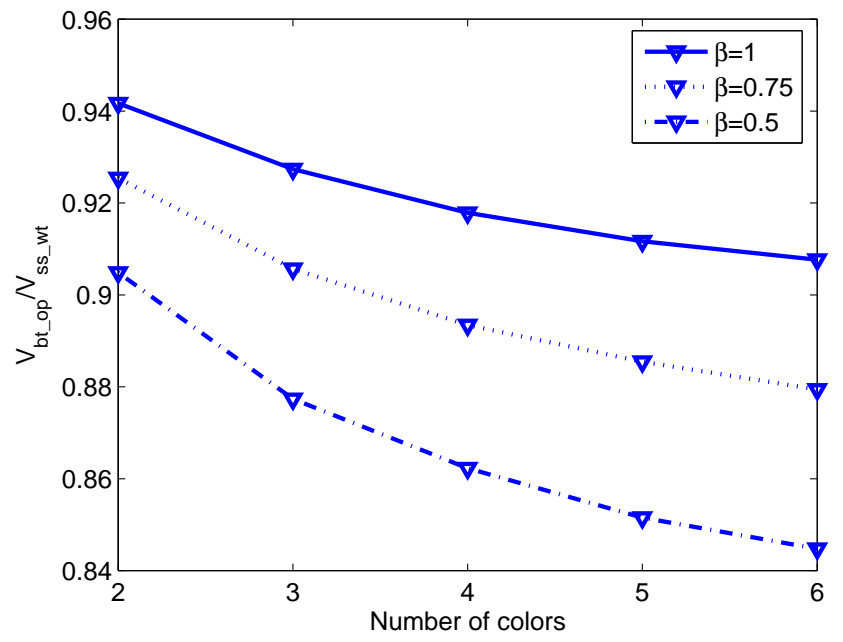

Figure 6.7: Emissions: optimal sequence in batch policy vs worst sequence in strictly sequencing policy 
Batch policy with optimal quality vs. strictly sequencing policy with optimal quality

Next we compare batch policy with strictly sequencing policy, both under optimal sequence schedule, $V_{b t \_o p} / V_{s s_{-} o p}$. As we expected, smaller magnitudes are obtained in this comparison, as shown in Figure 6.8. When $\beta=1$, there is about $5 \%$ reduction in emissions. When depreciation factor $\beta$ is smaller, the reduction is larger. For example, if $\beta=0.5$, such reduction is almost $9 \%$, which is a substantial reduction of emissions.

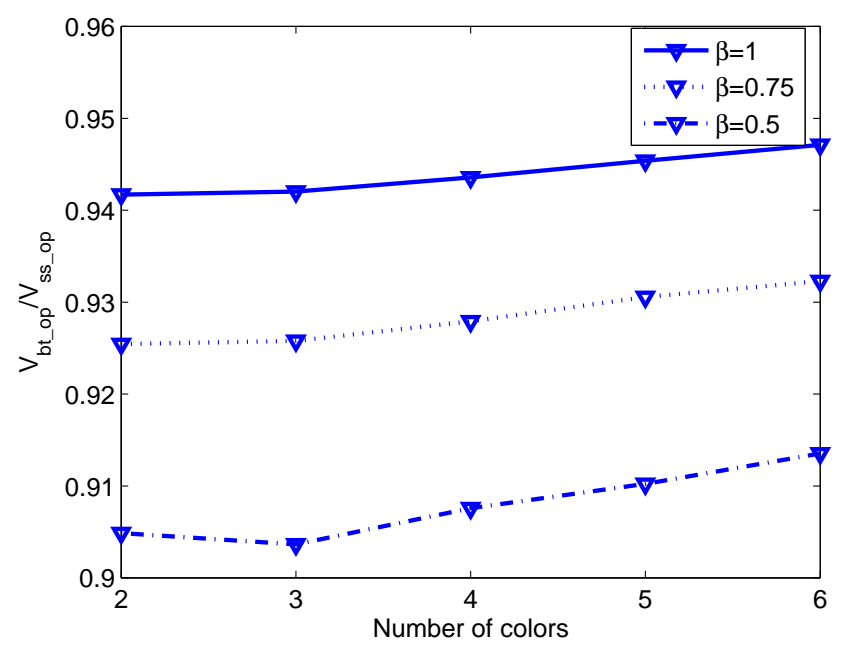

Figure 6.8: Emissions: optimal sequence in batch policy vs optimal sequence in strictly sequencing policy

\section{Batch policy with optimal quality vs. batch policy with worst quality}

Next the impact of sequencing on emissions is studied in batch production environment, i.e., we compare optimal and worst sequences with respect to quality in batch production, $V_{b t \_o p} / V_{b t \_w t}$. We observe that we can have almost $1 \%$ reduction in emission when no depreciation is assumed. When there lower quality during repaint, the reduction can be more than $2 \%$ (see Figure 6.9). These results are due to that 
the emissions during purges are significantly less than that during regular painting (through over spray). In this study, such a ratio, $\alpha$, is selected as $1 / 50$.

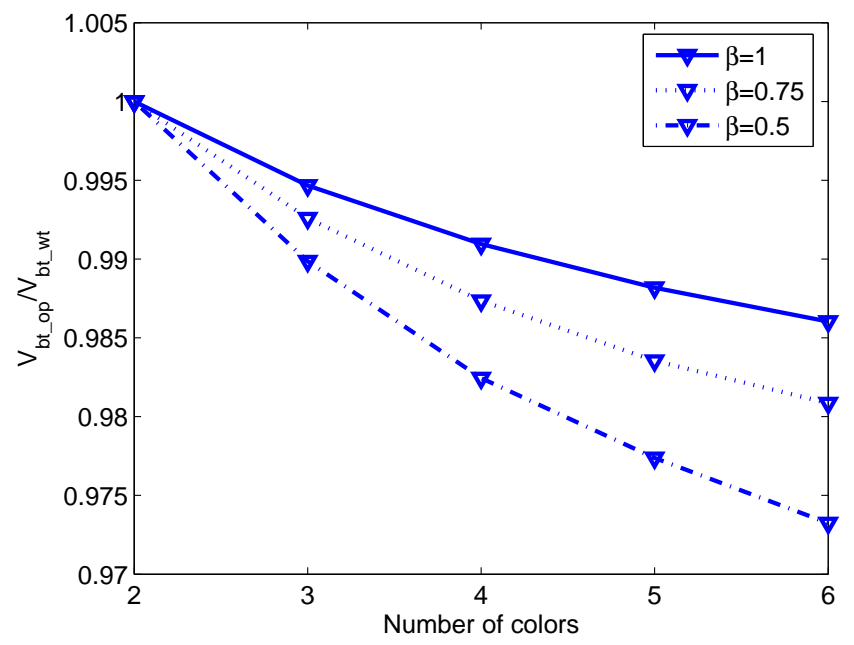

Figure 6.9: Emission: optimal sequence vs worst sequence in batch policy

\section{Batch policy with optimal quality vs. randomly mixed policy with average quality}

Finally, randomly mixed scenario is investigated by comparing with optimal batch and sequencing policy. Figure 6.10 illustrates the comparison results between $V_{b t \_o p}$ and $V_{r n d}$. As one can see, the emission reductions that can be obtained are from $4 \%$ to $9 \%$ for different $\beta$ s.

Similar to the energy reduction case, the above results again validate the importance of introducing optimal batch and sequencing policy to improve the quality performance and reduce atmospheric emissions.

\subsection{Summary}

In this chapter, we introduce application of optimal batch and scheduling policies to reduce energy consumption and atmospheric emissions in automotive paint shops. We 


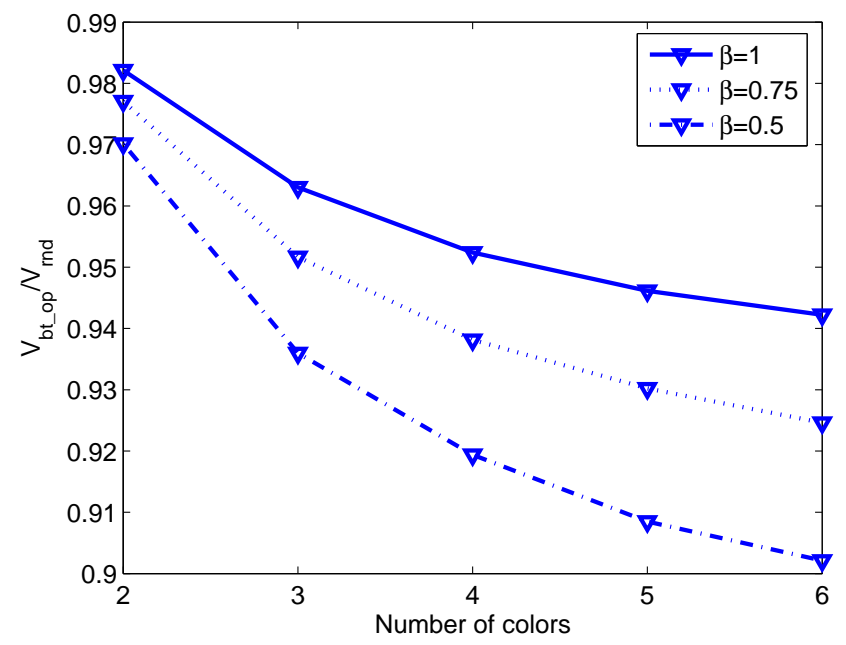

Figure 6.10: Emissions: optimal sequence in batch policy vs randomly mixed policy show that the energy consumption and Carbon emissions can be reduced significantly. Thus, without any equipment investment or process changes, energy efficiency can be improved substantially, and emissions adverse to the environment can be reduced significantly. Therefore, the method presented here provides another approach to achieve sustainable manufacturing in automotive paint shops. 


\section{CHAPTER 7}

\section{CASE STUDY}

The method introduced in the previous chapters has been applied at an automotive paint shop to evaluate paint quality and investigate potential improvement strategies. In the painting system under consideration, the painting booth is capable of painting any colors. An inspection station is next to the painting operation to check the paint quality of every vehicle. Seven color options are available, and we denote them as colors A to G.

The good job ratio of this painting systems is measured with $P(g)=0.8278$. Since the system is operating at a randomly mixed policy without specific sequencing, the results obtained in paper [21] are used to model the system quality. Based on the historical data, we calculate the quality failure and repair probabilities, $\lambda_{i j}$ and $\mu_{i j}$. Using them, we obtain the calculated good job ratio as 0.8245 , which is only $0.39 \%$ difference with the measured one. Thus, the model is validated. (Note that in order to confidentiality, the data presented here has been modified, however, the accuracy is preserved.)

Next, using the calculated transition probabilities, we obtain the quality bottleneck sequence as 'A-D-G-C-E-F-B', which leads to $P(g)=0.7356$, much lower than current one. Then, we investigate how to improve system quality through resequencing. 
- First, we select one sequence 'A-F-G-C-D-E-B', with $\sum e_{i j}=5.5884$ and $P(g)=$ 0.8235 , which is close to current system measurement. We use this sequence as a replacement of current system operating under sequence policy.

- Then, we randomly select another sequence 'A-F-E-G-B-C-D', which has $\sum e_{i j}=$ 5.9837, and $P(g)=0.8564$. It is clear that larger $\sum e_{i j}$ results in higher $P(g)$.

- Next, we check how to change the order of colors so that quality can be improved. For the sequence 'A-F-G-C-D-E-B', we switch the order of 'F' and 'G', and obtain a new sequence 'A-G-F-C-D-E-B'. This results in $\sum e_{i j}=6.0936$ and $P(g)=0.8857$. This implies a $7.55 \%$ improvement can be achieved by simply switching the order of two colors.

Finally, the bottleneck transitions are identified to improve product quality. Using the n-BN-t and p-BN-t indicators, we identify $\lambda_{22}$ and $\mu_{22}$ as the negative and positive bottleneck transitions, respectively. This result is also verified by calculating $\Delta P(g) / \Delta \lambda_{i j}$ and $\Delta P(g) / \Delta \mu_{i j}$ numerically. By improving $\lambda_{22}$ from 0.2160 to 0.2 , the paint quality is increased to 0.8427 . Or by improving $\mu_{22}$ from 0.7215 to 0.75 , the product quality is upgraded to 0.8394 . Therefore, quality improvement can be achieved by focusing on mitigating the bottleneck transitions.

The above study indicates that the presented method can be used for quality improvement in flexible manufacturing systems. 


\section{CHAPTER 8 CONCLUSIONS}

Flexible manufacturing systems have been studied extensively during the last thirty year. Most of the studies emphasize on the tradeoffs between productivity and flexibility, while the issue of product quality is usually neglected. It is typically assumed that quality related issues have minimal impact. However, empirical evidences and analytical studies have shown that flexibility has a significant impact on quality. In addition, many flexible manufacturing systems implement batch productions to improve product quality by reducing product changes which may affect product quality

during changeover. However, no analytical studies are available to address quality in batch production enviromment. Therefore, there is a critical need to fully understand the coupling between flexibility and quality in batch productions.

In this research, an analytical method based on Markov chain model is presented to evaluate the quality performance in a flexible manufacturing system with batch production. A closed formula is derived to quantify quality performance which is defined as the probability of producing a good part. Structural properties to address the transitions and appropriate batch size are discussed. Counter intuitively, monotonicity with respect to transitions and batch size does not always hold. It is shown that when quality failure probability is relative high, or repair probability relatively low, or quality efficiency significantly low, monotonicity may not stand. In addition, 
more monotonic cases are observed when batch size increases. Necessary conditions of transitions and batch sizes are provided to avoid possible negative effect due to non-monotonicity when carrying out continuous improvement.

In addition, different product sequencing and batch policies may result in different quality. Then, how to determine an appropriate sequence or batch policy? How to select the optimal sequence with respect to quality? Will the quality characteristics change under different policies? Answers to these questions can provide guidance for system design and operation to achieve better product quality. Based on the analytical model, the quality performance in batch and strictly sequencing policies is studied. The optimal sequences leading to best quality under both policies are obtained, the comparisons between different sequences under both policies are carried out, and finally the comparison between two policies is studied. It is shown that the optimality and superiority of a product sequence in one policy still hold in the other one. In particular, batch policy outperforms strictly sequencing policy if the total quality efficiency without product switch is better than the total quality efficiency with product switch, and vice versa.

To improve the quality performance from the perspective of product sequencing, the notions of quality improvability and bottleneck sequence are introduced. A flexible system is improvable in terms of quality with respect to sequencing if there exists another sequence which renders better quality, otherwise it is unimprovable (i.e., optimal). In addition, quality bottleneck sequence is defined as the one that impedes the product quality in the strongest manner, i.e., the sequence that leads to the worst quality among all possible ones. Indicators for quality improvability and bottleneck 
sequence, based on the data available on the factory floor rather than complicated calculations of quality performance, are developed. Such developments provide a simple tool for production engineers and managers to design the appropriate sequence to achieve higher quality in flexible manufacturing systems.

Identifying the quality bottleneck transition is another effective way to improve the quality of a flexible manufacturing system. Similar with bottleneck sequence, the bottleneck transition is defined as the one that impedes quality performance in the strongest manner. The sensitivity of quality performance with respect to quality failure and repair probabilities is investigated and quality bottleneck transition indicators are proposed based on the data collected on the factory floor.

In automotive assembly plants, the largest amount of energy consumption and atmospheric emissions is in paint shop. Optimizing the energy usage to pursue maximum energy savings, and reducing of Carbon Dioxide equivalent emissions are of significant importance in automotive paint shops. Instead of inventing new chemicals, new painting processes or new control systems in painting booths and ovens, an optimal batch and scheduling procedure of vehicles to achieve the goal of energy and emission reduction is developed. Specifically, by selecting appropriate batch and sequence policies, the paint quality can be improved and repaints can be reduced so that less material and energy will be consumed, and fewer atmospheric emissions will be generated. It is shown that such scheduling and control method can lead to significant energy savings and emission reduction with no extra investment, nor changes to existing painting processes.

The presented method has been applied at an automotive paint shop to evaluate 
paint quality and investigate potential improvement strategies. Based on historical inspection data, quality failure and repair probabilities are calculated. Given the transition probabilities and product sequence, the model is validated with good accuracy. Then the quality bottleneck sequence is identified, and how to improve product quality through resequencing is introduced. Finally, the negative and positive bottleneck transitions are identified. Improving them can lead to substantial improvement in quality.

The model introduced here can be used to evaluate quality and investigate sequencing policies in many flexible systems with batch productions. It provides industrial engineers and operation managers a quantitative tool for continuous improvement on product quality in flexible manufacturing environment. However, it still has limitations. It is more suitable for manufacturing systems with $100 \%$ inspections such that substantial amount of data can be obtained to calculate transition probabilities. In addition, when the number of products is large, the computation efficiency of quality performance might be a problem. In such a case, the bottleneck indicators have more advantages.

Future work can be directed to the following issues:

- First, consider the reprocessing cases. For example, at an automotive paint shop, a vehicle is inspected defective and might be sent to the same paint booth again for repaints. In this case, transition probabilities of this type usually change during repaints compared with first painting. Besides, resequencing problem appears each time a repaint is required. 
- Second, continue our application effort to apply the methods on the factory floor, e.g., automotive paint shops, for model validation and quality improvement.

- Finally, integrate this method into quality control and assurance strategies. 


\section{APPENDICES}

\section{Appendix A: Proofs of Chapter 3}

\section{Proof of Theorem 3.1;}

It can be shown that

$$
\begin{aligned}
& P\left(g_{11}\right)+P\left(d_{11}\right)=P\left(g_{22}\right)+P\left(d_{22}\right), \\
& P\left(g_{12}\right)+P\left(d_{12}\right)=P\left(g_{11}\right)+P\left(d_{11}\right), \\
& P\left(g_{21}\right)+P\left(d_{21}\right)=P\left(g_{12}\right)+P\left(d_{12}\right) .
\end{aligned}
$$

Therefore

$$
P\left(g_{11}\right)+P\left(d_{11}\right)=P\left(g_{12}\right)+P\left(d_{12}\right)=P\left(g_{21}\right)+P\left(d_{21}\right)=P\left(g_{22}\right)+P\left(d_{22}\right)=\frac{1}{4}
$$

Then

$$
\begin{aligned}
P\left(g_{11}\right) & =\mu_{12}\left[\frac{1}{4}-P\left(g_{22}\right)\right]+\left(1-\lambda_{12}\right) P\left(g_{22}\right) \\
& =\frac{1}{4} \mu_{12}+\left(1-\lambda_{12}-\mu_{12}\right) P\left(g_{22}\right) \\
P\left(g_{12}\right) & =\mu_{11}\left[\frac{1}{4}-P\left(g_{11}\right)\right]+\left(1-\lambda_{11}\right) P\left(g_{11}\right) \\
& =\frac{1}{4} \mu_{11}+\left(1-\lambda_{11}-\mu_{11}\right) P\left(g_{11}\right) \\
& =\frac{1}{4} \mu_{11}+\frac{1}{4} \mu_{12}\left(1-\lambda_{11}-\mu_{11}\right)+\left(1-\lambda_{12}-\mu_{12}\right)\left(1-\lambda_{11}-\mu_{11}\right) P\left(g_{22}\right), \\
P\left(g_{21}\right) & =\left(1-\lambda_{21}\right) P\left(g_{12}\right)+\mu_{21}\left[\frac{1}{4}-P\left(g_{12}\right)\right] \\
& =\frac{1}{4} \mu_{21}+\left(1-\lambda_{21}-\mu_{21}\right) P\left(g_{12}\right) \\
& =\frac{1}{4} \mu_{21}+\frac{1}{4} \mu_{11}\left(1-\lambda_{21}-\mu_{21}\right)+\frac{1}{4} \mu_{12}\left(1-\lambda_{21}-\mu_{21}\right)\left(1-\lambda_{11}-\mu_{11}\right)
\end{aligned}
$$




$$
\begin{aligned}
& +\left(1-\lambda_{21}-\mu_{21}\right)\left(1-\lambda_{12}-\mu_{12}\right)\left(1-\lambda_{11}-\mu_{11}\right) P\left(g_{22}\right), \\
P\left(g_{22}\right)= & \mu_{22}\left[\frac{1}{4}-P\left(g_{21}\right)\right]+\left(1-\lambda_{22}\right) P\left(g_{21}\right) \\
= & \frac{1}{4} \mu_{22}+\left(1-\lambda_{22}-\mu_{22}\right) P\left(g_{21}\right) \\
= & \frac{1}{4} \mu_{22}+\frac{1}{4} \mu_{21}\left(1-\lambda_{22}-\mu_{22}\right)+\frac{1}{4} \mu_{11}\left(1-\lambda_{21}-\mu_{21}\right)\left(1-\lambda_{22}-\mu_{22}\right) \\
& +\frac{1}{4} \mu_{12}\left(1-\lambda_{21}-\mu_{21}\right)\left(1-\lambda_{11}-\mu_{11}\right)\left(1-\lambda_{22}-\mu_{22}\right) \\
& +\left(1-\lambda_{21}-\mu_{21}\right)\left(1-\lambda_{22}-\mu_{22}\right)\left(1-\lambda_{11}-\mu_{11}\right)\left(1-\lambda_{12}-\mu_{12}\right) P\left(g_{22}\right) .
\end{aligned}
$$

Furthermore

$$
\begin{aligned}
P\left(g_{22}\right)= & \left(\mu_{22}+\mu_{21}\left(1-\lambda_{22}-\mu_{22}\right)+\mu_{11}\left(1-\lambda_{21}-\mu_{21}\right)\left(1-\lambda_{22}-\mu_{22}\right)\right. \\
& \left.+\mu_{12}\left(1-\lambda_{21}-\mu_{21}\right)\left(1-\lambda_{11}-\mu_{11}\right)\left(1-\lambda_{22}-\mu_{22}\right)\right) \\
& /\left(4\left[1-\left(1-\lambda_{21}-\mu_{21}\right)\left(1-\lambda_{22}-\mu_{22}\right)\left(1-\lambda_{11}-\mu_{11}\right)\left(1-\lambda_{12}-\mu_{12}\right)\right]\right) .
\end{aligned}
$$

Hence, we can obtain the overall quality performance

$$
\begin{aligned}
P(g)= & P\left(g_{11}\right)+P\left(g_{12}\right)+P\left(g_{21}\right)+P\left(g_{22}\right) \\
= & \frac{1}{4} \mu_{12}+\left(1-\lambda_{12}-\mu_{12}\right) P\left(g_{22}\right)+\frac{1}{4} \mu_{11}+\frac{1}{4} \mu_{12}\left(1-\lambda_{11}-\mu_{11}\right) \\
& +\left(1-\lambda_{12}-\mu_{12}\right)\left(1-\lambda_{11}-\mu_{11}\right) P\left(g_{22}\right)+\frac{1}{4} \mu_{21}+\frac{1}{4} \mu_{11}\left(1-\lambda_{21}-\mu_{21}\right) \\
& +\frac{1}{4} \mu_{12}\left(1-\lambda_{21}-\mu_{21}\right)\left(1-\lambda_{11}-\mu_{11}\right)+P\left(g_{22}\right) \\
& +\left(1-\lambda_{21}-\mu_{21}\right)\left(1-\lambda_{12}-\mu_{12}\right)\left(1-\lambda_{11}-\mu_{11}\right) P\left(g_{22}\right) \\
& \frac{1}{4}\left[\mu_{11}+\mu_{21}+\mu_{12}+\mu_{11}\left(1-\lambda_{21}-\mu_{21}\right)+\mu_{12}\left(1-\lambda_{11}-\mu_{11}\right)\right. \\
& \left.+\mu_{12}\left(1-\lambda_{21}-\mu_{21}\right)\left(1-\lambda_{11}-\mu_{11}\right)\right]+P\left(g_{22}\right)\left[1+1-\lambda_{12}-\mu_{12}\right. \\
& +\left(1-\lambda_{12}-\mu_{12}\right)\left(1-\lambda_{11}-\mu_{11}\right)+\left(1-\lambda_{21}-\mu_{21}\right)\left(1-\lambda_{11}-\mu_{11}\right) \\
& \left.\left(1-\lambda_{12}-\mu_{12}\right)\right]
\end{aligned}
$$




$$
\begin{aligned}
& =\frac{1}{4}\left[\mu_{21}+\mu_{12}+\mu_{11}\left(2-\lambda_{21}-\mu_{21}\right)+\mu_{12}\left(1-\lambda_{11}-\mu_{11}\right)\left(2-\lambda_{21}-\mu_{21}\right)\right] \\
& +P\left(g_{22}\right)\left[2-\lambda_{12}-\mu_{12}+\left(1-\lambda_{11}-\mu_{11}\right)\left(2-\lambda_{21}-\mu_{21}\right)\left(1-\lambda_{12}-\mu_{12}\right)\right] \\
& =\frac{1}{4}\left[\mu_{21}+\mu_{12}+\left[\mu_{11}+\mu_{12}\left(1-\lambda_{11}-\mu_{11}\right)\right]\left(2-\lambda_{21}-\mu_{21}\right)\right] \\
& +P\left(g_{22}\right)\left[2-\lambda_{12}-\mu_{12}+\left(1-\lambda_{11}-\mu_{11}\right)\left(1-\lambda_{12}-\mu_{12}\right)\left(2-\lambda_{21}-\mu_{21}\right)\right] \\
& =\frac{1}{4}\left[\mu_{21}+\mu_{12}+\left[\mu_{11}+\mu_{12}\left(1-\lambda_{11}-\mu_{11}\right)\right]\left(2-\lambda_{21}-\mu_{21}\right)\right] \\
& +\left[2-\lambda_{12}-\mu_{12}+\left(1-\lambda_{11}-\mu_{11}\right)\left(1-\lambda_{12}-\mu_{12}\right)\left(2-\lambda_{21}-\mu_{21}\right)\right] \\
& \cdot\left[\mu_{22}+\mu_{21}\left(1-\lambda_{22}-\mu_{22}\right)+\mu_{11}\left(1-\lambda_{21}-\mu_{21}\right)\left(1-\lambda_{22}-\mu_{22}\right)\right. \\
& \left.+\mu_{12}\left(1-\lambda_{21}-\mu_{21}\right)\left(1-\lambda_{11}-\mu_{11}\right)\left(1-\lambda_{22}-\mu_{22}\right)\right] \\
& /\left[4\left[1-\left(1-\lambda_{21}-\mu_{21}\right)\left(1-\lambda_{22}-\mu_{22}\right)\left(1-\lambda_{11}-\mu_{11}\right)\left(1-\lambda_{12}-\mu_{12}\right)\right]\right] \\
& =\left[\mu_{11}\left(1-\lambda_{21}-\mu_{21}\right)\left[1+\left(1-\lambda_{22}-\mu_{22}\right)\left(1-\lambda_{12}-\mu_{12}\right)\right]\right. \\
& +\mu_{12}\left(1-\lambda_{11}-\mu_{11}\right)\left[1+\left(1-\lambda_{22}-\mu_{22}\right)\left(1-\lambda_{21}-\mu_{21}\right)\right] \\
& +\mu_{21}\left(1-\lambda_{22}-\mu_{22}\right)\left[1+\left(1-\lambda_{12}-\mu_{12}\right)\left(1-\lambda_{11}-\mu_{11}\right)\right] \\
& +\mu_{22}\left(1-\lambda_{12}-\mu_{12}\right)\left[1+\left(1-\lambda_{11}-\mu_{11}\right)\left(1-\lambda_{21}-\mu_{21}\right)\right] \\
& +\mu_{21}\left[1+\left(1-\lambda_{22}-\mu_{22}\right)\left(1-\lambda_{12}-\mu_{12}\right)\right] \\
& +\mu_{12}\left[1+\left(1-\lambda_{11}-\mu_{11}\right)\left(1-\lambda_{21}-\mu_{21}\right)\right] \\
& +\mu_{11}\left[1+\left(1-\lambda_{22}-\mu_{22}\right)\left(1-\lambda_{21}-\mu_{21}\right)\right] \\
& \left.+\mu_{22}\left[1+\left(1-\lambda_{12}-\mu_{12}\right)\left(1-\lambda_{11}-\mu_{11}\right)\right]\right] \\
& /\left[4\left[1-\left(1-\lambda_{21}-\mu_{21}\right)\left(1-\lambda_{22}-\mu_{22}\right)\left(1-\lambda_{11}-\mu_{11}\right)\left(1-\lambda_{12}-\mu_{12}\right)\right]\right] \\
& =\left[\left[\mu_{21}+\mu_{11}\left(1-\lambda_{21}-\mu_{21}\right)\right]\left[1+\left(1-\lambda_{22}-\mu_{22}\right)\left(1-\lambda_{12}-\mu_{12}\right)\right]\right. \\
& +\left[\mu_{11}+\mu_{12}\left(1-\lambda_{11}-\mu_{11}\right)\right]\left[1+\left(1-\lambda_{22}-\mu_{22}\right)\left(1-\lambda_{21}-\mu_{21}\right)\right]
\end{aligned}
$$




$$
\begin{aligned}
& +\left[\mu_{22}+\mu_{21}\left(1-\lambda_{22}-\mu_{22}\right)\right]\left[1+\left(1-\lambda_{12}-\mu_{12}\right)\left(1-\lambda_{11}-\mu_{11}\right)\right] \\
& \left.+\left[\mu_{12}+\mu_{22}\left(1-\lambda_{12}-\mu_{12}\right)\right]\left[1+\left(1-\lambda_{11}-\mu_{11}\right)\left(1-\lambda_{21}-\mu_{21}\right)\right]\right] \\
& /\left[4\left[1-\left(1-\lambda_{21}-\mu_{21}\right)\left(1-\lambda_{22}-\mu_{22}\right)\left(1-\lambda_{11}-\mu_{11}\right)\left(1-\lambda_{12}-\mu_{12}\right)\right]\right] .
\end{aligned}
$$

Let

$$
\begin{aligned}
\mathcal{A} & =\left[\mu_{21}+\mu_{11}\left(1-\lambda_{21}-\mu_{21}\right)\right]\left[1+\left(1-\lambda_{22}-\mu_{22}\right)\left(1-\lambda_{12}-\mu_{12}\right)\right], \\
\mathcal{B} & =\left[\mu_{11}+\mu_{12}\left(1-\lambda_{11}-\mu_{11}\right)\right]\left[1+\left(1-\lambda_{22}-\mu_{22}\right)\left(1-\lambda_{21}-\mu_{21}\right)\right], \\
\mathcal{C} & =\left[\mu_{21}+\mu_{21}\left(1-\lambda_{22}-\mu_{22}\right)\right]\left[1+\left(1-\lambda_{12}-\mu_{12}\right)\left(1-\lambda_{11}-\mu_{11}\right)\right], \\
\mathcal{D} & =\left[\mu_{12}+\mu_{22}\left(1-\lambda_{12}-\mu_{12}\right)\right]\left[1+\left(1-\lambda_{11}-\mu_{11}\right)\left(1-\lambda_{21}-\mu_{21}\right)\right], \\
\mathcal{F} & =\left(1-\lambda_{21}-\mu_{21}\right)\left(1-\lambda_{22}-\mu_{22}\right)\left(1-\lambda_{11}-\mu_{11}\right)\left(1-\lambda_{12}-\mu_{12}\right) .
\end{aligned}
$$

We have

$$
P(g)=\frac{\mathcal{A}+\mathcal{B}+\mathcal{C}+\mathcal{D}}{4(1-\mathcal{F})} .
$$

\section{Proof of Corollary 3.1:}

$$
\begin{aligned}
P(g)= & {\left[2\left[1+\left(1-\lambda_{11}-\mu_{11}\right)\left(1-\lambda_{21}-\mu_{21}\right)\right]\left[\mu_{21}+\mu_{11}\left(1-\lambda_{21}-\mu_{21}\right)\right]\right.} \\
& \left.+2\left[1+\left(1-\lambda_{11}-\mu_{11}\right)\left(1-\lambda_{21}-\mu_{21}\right)\right]\left[\mu_{11}+\mu_{21}\left(1-\lambda_{11}-\mu_{11}\right)\right]\right] \\
& /\left[4\left[1-\left(1-\lambda_{11}-\mu_{11}\right)^{2}\left(1-\lambda_{21}-\mu_{21}\right)^{2}\right]\right] \\
= & {\left[[ 1 + ( 1 - \lambda _ { 1 1 } - \mu _ { 1 1 } ) ( 1 - \lambda _ { 2 1 } - \mu _ { 2 1 } ) ] \left[\mu_{21}+\mu_{11}\left(1-\lambda_{21}-\mu_{21}\right)+\mu_{11}\right.\right.} \\
& \left.\left.+\mu_{21}\left(1-\lambda_{11}-\mu_{11}\right)\right]\right] /\left[2\left[1-\left(1-\lambda_{11}-\mu_{11}\right)\left(1-\lambda_{21}-\mu_{21}\right)\right]\right. \\
& \left.\cdot\left[1+\left(1-\lambda_{11}-\mu_{11}\right)\left(1-\lambda_{21}-\mu_{21}\right)\right]\right] \\
= & \frac{\mu_{11}+\mu_{21}+\mu_{11}\left(1-\lambda_{21}-\mu_{21}\right)+\mu_{21}\left(1-\lambda_{11}-\mu_{11}\right)}{2\left[1-\left(1-\lambda_{11}-\mu_{11}\right)\left(1-\lambda_{21}-\mu_{21}\right)\right]}
\end{aligned}
$$




$$
=\frac{\mu_{11}\left(2-\lambda_{21}-\mu_{21}\right)+\mu_{21}\left(2-\lambda_{11}-\mu_{11}\right)}{2\left[1-\left(1-\lambda_{11}-\mu_{11}\right)\left(1-\lambda_{21}-\mu_{21}\right)\right]} .
$$

\section{Proof of Corollary 3.2 :}

Since $0<\lambda_{11}, \lambda_{21}, \mu_{11}, \mu_{21}<1$, we have

$$
\begin{aligned}
& -1<1-\lambda_{11}-\mu_{11}<1 \\
& -1<1-\lambda_{21}-\mu_{21}<1
\end{aligned}
$$

In order to analyze the monotonicity with respect to $\lambda_{11}, \lambda_{21}, \mu_{11}, \mu_{21}$, we derive the their corresponding partial differentials from Corollary 3.1 above.

$$
\begin{aligned}
& \frac{\partial P(g)}{\partial \lambda_{11}}=-\frac{2 \mu_{11}\left[1-\left(1-\lambda_{11}-\mu_{11}\right)\left(1-\lambda_{21}-\mu_{21}\right)\right]}{4\left[1-\left(1-\lambda_{11}-\mu_{11}\right)\left(1-\lambda_{21}-\mu_{21}\right)\right]^{2}} \\
& -\frac{2\left(1-\lambda_{21}-\mu_{21}\right)\left[\mu_{11}\left(2-\lambda_{21}-\mu_{21}\right)+\mu_{21}\left(2-\lambda_{11}-\mu_{11}\right)\right]}{4\left[1-\left(1-\lambda_{11}-\mu_{11}\right)\left(1-\lambda_{21}-\mu_{21}\right)\right]^{2}} \\
& =-\frac{2\left[\mu_{21}+\mu_{11}\left(1-\lambda_{21}-\mu_{21}\right)\right]\left(2-\lambda_{21}-\mu_{21}\right)}{4\left[1-\left(1-\lambda_{11}-\mu_{11}\right)\left(1-\lambda_{21}-\mu_{21}\right)\right]^{2}} \\
& =-\frac{2\left[\mu_{21}\left(1-\mu_{11}\right)+\mu_{11}\left(1-\lambda_{21}\right)\right]\left(2-\lambda_{21}-\mu_{21}\right)}{4\left[1-\left(1-\lambda_{11}-\mu_{11}\right)\left(1-\lambda_{21}-\mu_{21}\right)\right]^{2}} \\
& <0 \\
& \frac{\partial P(g)}{\partial \lambda_{21}}=-\frac{2 \mu_{11}\left[1-\left(1-\lambda_{11}-\mu_{11}\right)\left(1-\lambda_{21}-\mu_{21}\right)\right]}{4\left[1-\left(1-\lambda_{11}-\mu_{11}\right)\left(1-\lambda_{21}-\mu_{21}\right)\right]^{2}} \\
& -\frac{2\left(1-\lambda_{11}-\mu_{11}\right)\left[\mu_{11}\left(2-\lambda_{21}-\mu_{21}\right)+\mu_{21}\left(2-\lambda_{11}-\mu_{11}\right)\right]}{4\left[1-\left(1-\lambda_{11}-\mu_{11}\right)\left(1-\lambda_{21}-\mu_{21}\right)\right]^{2}} \\
& =-\frac{2\left[\mu_{11}+\mu_{21}\left(1-\lambda_{11}-\mu_{11}\right)\right]\left(2-\lambda_{11}-\mu_{11}\right)}{4\left[1-\left(1-\lambda_{11}-\mu_{11}\right)\left(1-\lambda_{21}-\mu_{21}\right)\right]^{2}} \\
& =-\frac{2\left[\mu_{11}\left(1-\mu_{21}\right)+\mu_{21}\left(1-\lambda_{11}\right)\right]\left(2-\lambda_{11}-\mu_{11}\right)}{4\left[1-\left(1-\lambda_{11}-\mu_{11}\right)\left(1-\lambda_{21}-\mu_{21}\right)\right]^{2}} \\
& <0 \\
& \frac{\partial P(g)}{\partial \mu_{11}}=\frac{2\left(2-\lambda_{21}-2 \mu_{21}\right)\left[1-\left(1-\lambda_{11}-\mu_{11}\right)\left(1-\lambda_{21}-\mu_{21}\right)\right]}{4\left[1-\left(1-\lambda_{11}-\mu_{11}\right)\left(1-\lambda_{21}-\mu_{21}\right)\right]^{2}}
\end{aligned}
$$




$$
\begin{aligned}
& -\frac{2\left(1-\lambda_{21}-\mu_{21}\right)\left[\mu_{11}\left(2-\lambda_{21}-\mu_{21}\right)+\mu_{21}\left(2-\lambda_{11}-\mu_{11}\right)\right]}{4\left[1-\left(1-\lambda_{11}-\mu_{11}\right)\left(1-\lambda_{21}-\mu_{21}\right)\right]^{2}} \\
= & \frac{2\left[\lambda_{21}+\lambda_{11}\left(1-\lambda_{21}-\mu_{21}\right)\right]\left(2-\lambda_{21}-\mu_{21}\right)}{4\left[1-\left(1-\lambda_{11}-\mu_{11}\right)\left(1-\lambda_{21}-\mu_{21}\right)\right]^{2}} \\
= & \frac{2\left[\lambda_{21}\left(1-\lambda_{11}\right)+\lambda_{11}\left(1-\mu_{21}\right)\right]\left(2-\lambda_{21}-\mu_{21}\right)}{4\left[1-\left(1-\lambda_{11}-\mu_{11}\right)\left(1-\lambda_{21}-\mu_{21}\right)\right]^{2}} \\
> & 0, \\
\frac{\partial P(g)}{\partial \mu_{21}}= & \frac{2\left(2-\lambda_{11}-2 \mu_{11}\right)\left[1-\left(1-\lambda_{11}-\mu_{11}\right)\left(1-\lambda_{21}-\mu_{21}\right)\right]}{4\left[1-\left(1-\lambda_{11}-\mu_{11}\right)\left(1-\lambda_{21}-\mu_{21}\right)\right]^{2}} \\
& -\frac{2\left(1-\lambda_{11}-\mu_{11}\right)\left[\mu_{11}\left(2-\lambda_{21}-\mu_{21}\right)+\mu_{21}\left(2-\lambda_{11}-\mu_{11}\right)\right]}{4\left[1-\left(1-\lambda_{11}-\mu_{11}\right)\left(1-\lambda_{21}-\mu_{21}\right)\right]^{2}} \\
= & \frac{2\left[\lambda_{11}+\lambda_{21}\left(1-\lambda_{11}-\mu_{11}\right)\right]\left(2-\lambda_{21}-\mu_{21}\right)}{4\left[1-\left(1-\lambda_{11}-\mu_{11}\right)\left(1-\lambda_{21}-\mu_{21}\right)\right]^{2}} \\
= & \frac{2\left[\lambda_{11}\left(1-\lambda_{21}\right)+\lambda_{21}\left(1-\mu_{11}\right)\right]\left(2-\lambda_{21}-\mu_{21}\right)}{4\left[1-\left(1-\lambda_{11}-\mu_{11}\right)\left(1-\lambda_{21}-\mu_{21}\right)\right]^{2}} \\
> & 0 .
\end{aligned}
$$

\section{Proof of Corollary 3.3:}

$$
\begin{aligned}
& P\left(g_{b t}\right)-P\left(g_{s s}\right) \\
= & \frac{\mu_{11}+\mu_{21}+\mu_{11}\left(1-\lambda_{21}-\mu_{21}\right)+\mu_{21}\left(1-\lambda_{11}-\mu_{11}\right)}{2\left[1-\left(1-\lambda_{11}-\mu_{11}\right)\left(1-\lambda_{21}-\mu_{21}\right)\right]}-\frac{\mu_{21}}{\lambda_{21}+\mu_{21}} \\
= & \frac{2 \mu_{21}+2 \mu_{11}-\mu_{21}\left(\lambda_{11}+\mu_{11}\right)-\mu_{11}\left(\lambda_{21}+\mu_{21}\right)}{2\left[\lambda_{21}+\mu_{21}+\lambda_{11}+\mu_{11}-\left(\lambda_{21}+\mu_{21}\right)\left(\lambda_{11}+\mu_{11}\right)\right]}-\frac{\mu_{21}}{\lambda_{21}+\mu_{21}} \\
= & \frac{1}{2}\left[2 \mu_{21}\left(\lambda_{21}+\mu_{21}\right)+2 \mu_{11}\left(\lambda_{21}+\mu_{21}\right)-\mu_{21}\left(\lambda_{21}+\mu_{21}\right)\left(\lambda_{11}+\mu_{11}\right)\right. \\
& -\mu_{11}\left(\lambda_{21}+\mu_{21}\right)^{2}-2 \mu_{21}\left(\lambda_{21}+\mu_{21}\right)-2 \mu_{21}\left(\lambda_{11}+\mu_{11}\right) \\
& \left.+2 \mu_{21}\left(\lambda_{21}+\mu_{21}\right)\left(\lambda_{11}+\mu_{11}\right)\right] \\
& /\left[\left(\lambda_{21}+\mu_{21}\right)\left[\lambda_{21}+\mu_{21}+\lambda_{11}+\mu_{11}-\left(\lambda_{21}+\mu_{21}\right)\left(\lambda_{11}+\mu_{11}\right)\right]\right] \\
= & \frac{\mu_{11}\left(\lambda_{21}+\mu_{21}\right)\left(2-\lambda_{21}-\mu_{21}\right)-\mu_{21}\left(\lambda_{11}+\mu_{11}\right)\left(2-\lambda_{21}-\mu_{21}\right)}{\left(\lambda_{21}+\mu_{21}\right)\left[\lambda_{21}+\mu_{21}+\lambda_{11}+\mu_{11}-\left(\lambda_{21}+\mu_{21}\right)\left(\lambda_{11}+\mu_{11}\right)\right]} \\
= & \frac{\left(2-\lambda_{21}-\mu_{21}\right)\left(\lambda_{21} \mu_{11}-\lambda_{11} \mu_{21}\right)}{\left(\lambda_{21}+\mu_{21}\right)\left[\lambda_{21}+\mu_{21}+\lambda_{11}+\mu_{11}-\left(\lambda_{21}+\mu_{21}\right)\left(\lambda_{11}+\mu_{11}\right)\right]} .
\end{aligned}
$$


Since

$$
\begin{aligned}
& \lambda_{21}+\mu_{21}+\lambda_{11}+\mu_{11}-\left(\lambda_{21}+\mu_{21}\right)\left(\lambda_{11}+\mu_{11}\right) \\
= & \left(\sqrt{\lambda_{21}+\mu_{21}}-\sqrt{\lambda_{11}+\mu_{11}}\right)^{2} \\
+ & \sqrt{\left(\lambda_{11}+\mu_{11}\right)\left(\lambda_{21}+\mu_{21}\right)}\left(2-\sqrt{\left(\lambda_{11}+\mu_{11}\right)\left(\lambda_{21}+\mu_{21}\right)}\right),
\end{aligned}
$$

and

$$
\lambda_{21}+\mu_{21}<2, \quad \lambda_{11}+\mu_{11}<2,
$$

we obtain

$$
\lambda_{21}+\mu_{21}+\lambda_{11}+\mu_{11}>\left(\lambda_{21}+\mu_{21}\right)\left(\lambda_{11}+\mu_{11}\right) .
$$

If

$$
e_{11}>e_{21},
$$

i.e.,

$$
\lambda_{21} \mu_{11}>\lambda_{11} \mu_{21}
$$

we have $P\left(g_{b t}\right)>P\left(g_{s s}\right)$ and vice versa when $e_{11}<e_{21}$.

\section{Proof of Corollary 3.4:}

$$
\begin{aligned}
& P\left(g_{b t}\right)-P\left(g_{r}\right) \\
= & \frac{2 \mu_{21}+2 \mu_{11}-\mu_{21}\left(\lambda_{11}+\mu_{11}\right)-\mu_{11}\left(\lambda_{21}+\mu_{21}\right)}{2\left[1-\left(1-\lambda_{11}-\mu_{11}\right)\left(1-\lambda_{21}-\mu_{21}\right)\right]}-\frac{\mu_{11}+\mu_{21}}{\lambda_{11}+\mu_{11}+\lambda_{21}+\mu_{21}} \\
= & {\left[2 \mu_{11}\left(\lambda_{11}+\mu_{11}\right)+2 \mu_{11}\left(\lambda_{21}+\mu_{21}\right)+2 \mu_{21}\left(\lambda_{11}+\mu_{11}\right)+2 \mu_{21}\left(\lambda_{21}+\mu_{21}\right)\right.} \\
& -\mu_{11}\left(\lambda_{11}+\mu_{11}\right)\left(\lambda_{21}+\mu_{21}\right)-\mu_{11}\left(\lambda_{21}+\mu_{21}\right)^{2}-\mu_{21}\left(\lambda_{11}+\mu_{11}\right)^{2} \\
& -\mu_{21}\left(\lambda_{11}+\mu_{11}\right)\left(\lambda_{21}+\mu_{21}\right)-2 \mu_{11}\left(\lambda_{11}+\mu_{11}\right)-2 \mu_{11}\left(\lambda_{21}+\mu_{21}\right)
\end{aligned}
$$




$$
\begin{aligned}
& +2 \mu_{11}\left(\lambda_{11}+\mu_{11}\right)\left(\lambda_{21}+\mu_{21}\right)-2 \mu_{21}\left(\lambda_{11}+\mu_{11}\right)-2 \mu_{21}\left(\lambda_{21}+\mu_{21}\right) \\
+ & \left.2 \mu_{21}\left(\lambda_{11}+\mu_{11}\right)\left(\lambda_{21}+\mu_{21}\right)\right] \\
& /\left[2\left[1-\left(1-\lambda_{11}-\mu_{11}\right)\left(1-\lambda_{21}-\mu_{21}\right)\right] \cdot\left(\lambda_{11}+\mu_{11}+\lambda_{21}+\mu_{21}\right)\right] \\
= & {\left[\left(\mu_{11}+\mu_{21}\right)\left(\lambda_{11}+\mu_{11}\right)\left(\lambda_{21}+\mu_{21}\right)-\mu_{11}\left(\lambda_{21}+\mu_{21}\right)^{2}-\mu_{21}\left(\lambda_{11}+\mu_{11}\right)^{2}\right] } \\
& /\left[2\left[1-\left(1-\lambda_{11}-\mu_{11}\right)\left(1-\lambda_{21}-\mu_{21}\right)\right]\left(\lambda_{11}+\mu_{11}+\lambda_{21}+\mu_{21}\right)\right] \\
= & \frac{\left(\lambda_{11}+\mu_{11}-\lambda_{21}-\mu_{21}\right)\left(\lambda_{21} \mu_{11}-\lambda_{11} \mu_{21}\right)}{2\left[1-\left(1-\lambda_{11}-\mu_{11}\right)\left(1-\lambda_{21}-\mu_{21}\right)\right]\left(\lambda_{11}+\mu_{11}+\lambda_{21}+\mu_{21}\right)} .
\end{aligned}
$$

Therefore, if $\lambda_{21} \mu_{11}>\lambda_{11} \mu_{21}$, i.e., $e_{11}>e_{21}$, and in addition, $\lambda_{11}+\mu_{11}>\lambda_{21}+\mu_{21}$, then

$$
P\left(g_{b t}\right)>P\left(g_{r}\right)
$$

where the second condition implies that $\frac{\mu_{11}}{e_{11}}>\frac{\mu_{21}}{e_{21}}$, the repair probability $\mu_{11}$ is sufficiently high.

\section{Proof of Corollary 3.5 :}

From the transition equations, we can obtain

$$
P\left(g_{i j}\right)+P\left(d_{i j}\right)=\frac{1}{n k}, i=1, \ldots, n ; j=1, \ldots, k
$$

Replacing $P\left(d_{i j}\right)$ with $\frac{1}{n k}-P\left(g_{i j}\right)$ in the transition equations, we have for the equal products case

$$
\begin{aligned}
& P\left(g_{11}\right)=\left(1-\lambda_{21}-\mu_{21}\right) P\left(g_{n, k}\right)+\frac{\mu_{21}}{n k}, \\
& P\left(g_{i 1}\right)=\left(1-\lambda_{21}-\mu_{21}\right) P\left(g_{i-1, k}\right)+\frac{\mu_{21}}{n k}, i=2, \ldots, n \\
& P\left(g_{i j}\right)=\left(1-\lambda_{11}-\mu_{11}\right) P\left(g_{i, j-1}\right)+\frac{\mu_{11}}{n k}, i=1, \ldots, n ; j=2, \ldots, k .
\end{aligned}
$$


Therefore, for $i=1, \ldots, n$ and $j=2, \ldots, k$,

$$
\begin{aligned}
P\left(g_{i j}\right) & =\sum_{v=0}^{j-2} \frac{\mu_{11}}{n k}\left(1-\lambda_{11}-\mu_{11}\right)^{v}+\left(1-\lambda_{11}-\mu_{11}\right)^{j-1} P\left(g_{i 1}\right) \\
& =\frac{\mu_{11}}{n k} \cdot \frac{1-\left(1-\lambda_{11}-\mu_{11}\right)^{j-1}}{\lambda_{11}+\mu_{11}}+\left(1-\lambda_{11}-\mu_{11}\right)^{j-1} P\left(g_{i 1}\right) .
\end{aligned}
$$

Adding all the states of first products, we have

$$
\begin{aligned}
\sum_{i=1}^{n} P\left(g_{i 1}\right)= & \left(1-\lambda_{21}-\mu_{21}\right)\left(P\left(g_{n, k}\right)+\sum_{i=2}^{n} P\left(g_{i-1, k}\right)\right)+\frac{\mu_{21}}{k} \\
= & \left(1-\lambda_{21}-\mu_{21}\right)\left(\frac{\mu_{11}\left[1-\left(1-\lambda_{11}-\mu_{11}\right)^{k-1}\right]}{k\left(\lambda_{11}+\mu_{11}\right)}\right. \\
& \left.+\left(1-\lambda_{11}-\mu_{11}\right)^{k-1} \sum_{i=1}^{n} P\left(g_{i 1}\right)\right)+\frac{\mu_{21}}{k}
\end{aligned}
$$

Finally, we can obtain

$$
\sum_{i=1}^{n} P\left(g_{i 1}\right)=\frac{\mu_{21}\left(\lambda_{11}+\mu_{11}\right)+\mu_{11}\left(1-\lambda_{21}-\mu_{21}\right)\left[1-\left(1-\lambda_{11}-\mu_{11}\right)^{k-1}\right]}{k\left(\lambda_{11}+\mu_{11}\right)\left[1-\left(1-\lambda_{21}-\mu_{21}\right)\left(1-\lambda_{11}-\mu_{11}\right)^{k-1}\right]} .
$$

The overall quality performance is

$$
\begin{aligned}
P(g)= & \sum_{i=1}^{n} \sum_{j=1}^{k} P\left(g_{i j}\right) \\
= & \sum_{i=1}^{n}\left(P\left(g_{i 1}\right)+\sum_{j=2}^{k} P\left(g_{i j}\right)\right) \\
= & \sum_{i=1}^{n}\left[P\left(g_{i 1}\right)+\sum_{j=2}^{k}\left(\frac{\mu_{11}}{n k} \cdot \frac{1-\left(1-\lambda_{11}-\mu_{11}\right)^{j-1}}{\lambda_{11}+\mu_{11}}+\left(1-\lambda_{11}-\mu_{11}\right)^{j-1} P\left(g_{i 1}\right)\right)\right] \\
= & \sum_{i=1}^{n}\left[\frac{1-\left(1-\lambda_{11}-\mu_{11}\right)^{k}}{\lambda_{11}+\mu_{11}} P\left(g_{i 1}\right)+\frac{\mu_{11}}{n k\left(\lambda_{11}+\mu_{11}\right)}\left(k-\frac{1-\left(1-\lambda_{11}-\mu_{11}\right)^{k}}{\lambda_{11}+\mu_{11}}\right)\right] \\
= & \frac{1-\left(1-\lambda_{11}-\mu_{11}\right)^{k}}{\lambda_{11}+\mu_{11}} P\left(g_{i 1}\right)+\frac{\mu_{11}}{k\left(\lambda_{11}+\mu_{11}\right)}\left(k-\frac{1-\left(1-\lambda_{11}-\mu_{11}\right)^{k}}{\lambda_{11}+\mu_{11}}\right) \\
= & \frac{\mu_{11}}{k\left(\lambda_{11}+\mu_{11}\right)}\left(k-\frac{1-\left(1-\lambda_{11}-\mu_{11}\right)^{k}}{\lambda_{11}+\mu_{11}}\right)+\frac{1-\left(1-\lambda_{11}-\mu_{11}\right)^{k}}{\lambda_{11}+\mu_{11}} \\
& \cdot\left(\frac{\mu_{21}\left(\lambda_{11}+\mu_{11}\right)+\mu_{11}\left(1-\lambda_{21}-\mu_{21}\right)\left[1-\left(1-\lambda_{11}-\mu_{11}\right)^{k-1}\right]}{k\left(\lambda_{11}+\mu_{11}\right)\left[1-\left(1-\lambda_{21}-\mu_{21}\right)\left(1-\lambda_{11}-\mu_{11}\right)^{k-1}\right]}\right) \\
= & \frac{\mu_{11}}{\lambda_{11}+\mu_{11}}+\frac{\left(\lambda_{11} \mu_{21}-\lambda_{21} \mu_{11}\right)\left[1-\left(1-\lambda_{11}-\mu_{11}\right)^{k}\right]}{k\left(\lambda_{11}+\mu_{11}\right)^{2}\left[1-\left(1-\lambda_{21}-\mu_{21}\right)\left(1-\lambda_{11}-\mu_{11}\right)^{k-1}\right]} .
\end{aligned}
$$




\section{Proof of Proposition 3.1:}

Let $a=1-\lambda_{11}-\mu_{11}$ and $b=1-\lambda_{21}-\mu_{21}$, then we have

$$
\begin{aligned}
\frac{\partial P(g)}{\partial \lambda_{21}} & =\frac{-\mu_{11}\left(1-a^{k}\right)\left(1-b a^{k-1}\right)-\left(\lambda_{11} \mu_{21}-\lambda_{21} \mu_{11}\right)\left(1-a^{k}\right) a^{k-1}}{k(1-a)^{2}\left(1-b a^{k-1}\right)^{2}} \\
& =-\frac{\left(1-a^{k}\right)\left[\mu_{11}\left(1-a^{k-1}\right)+\mu_{21} a^{k-1}(1-a)\right]}{k(1-a)^{2}\left(1-b a^{k-1}\right)^{2}} .
\end{aligned}
$$

If $a=0$, the partial differential equation can be simplified and we can easily find $\frac{\partial P(g)}{\partial \lambda_{21}}<0$

If $a^{k-1}>0$, we can easily find $\frac{\partial P(g)}{\partial \lambda_{21}}<0$.

If $a^{k-1}<0$, that means $a<0$ and therefore $a^{k-2}>0$. The equation can be expressed as follows

$$
\begin{aligned}
\frac{\partial P(g)}{\partial \lambda_{21}} & =\frac{-\mu_{11}\left(1-a^{k}\right)\left(1-b a^{k-1}\right)-\left(\lambda_{11} \mu_{21}-\lambda_{21} \mu_{11}\right)\left(1-a^{k}\right) a^{k-1}}{k(1-a)^{2}\left(1-b a^{k-1}\right)^{2}} \\
& =-\frac{\left(1-a^{k}\right)\left[\mu_{11}\left(1-a^{k-1}\right)+\mu_{21} a^{k-1}(1-a)\right]}{k(1-a)^{2}\left(1-b a^{k-1}\right)^{2}} \\
& =-\frac{\left(1-a^{k}\right)(1-a)\left(\mu_{11} \cdot \sum_{m=0}^{k-2} a^{m}+\mu_{21} a^{k-1}\right)}{k(1-a)^{2}\left(1-b a^{k-1}\right)^{2}} \\
& =-\frac{\left(1-a^{k}\right)(1-a)\left(\mu_{11} \cdot \sum_{m=0}^{k-3} a^{m}+\mu_{11} a^{k-2}+\mu_{21} a^{k-1}\right)}{k(1-a)^{2}\left(1-b a^{k-1}\right)^{2}} \\
& =-\frac{\left(1-a^{k}\right)(1-a)\left(\mu_{11} \frac{1-a^{k-2}}{1-a}+\left(\mu_{11}+\mu_{21} a\right) a^{k-2}\right)}{k(1-a)^{2}\left(1-b a^{k-1}\right)^{2}} \\
& =-\frac{\left(1-a^{k}\right)(1-a)\left[\mu_{11} \frac{1-a^{k-2}}{1-a}+\left(\mu_{11}+\mu_{21}-\mu_{21} \lambda_{11}-\mu_{21} \mu_{11}\right) a^{k-2}\right]}{k(1-a)^{2}\left(1-b a^{k-1}\right)^{2}} \\
& =-\frac{\left(1-a^{k}\right)(1-a)\left[\mu_{11} \frac{1-a^{k-2}}{1-a}+\left(\mu_{11}\left(1-\mu_{21}\right)+\mu_{21}\left(1-\lambda_{11}\right)\right) a^{k-2}\right]}{k(1-a)^{2}\left(1-b a^{k-1}\right)^{2}} \\
& <0 .
\end{aligned}
$$

Similar derivation for $\frac{\partial P(g)}{\partial \mu_{21}}>0$

$$
\begin{aligned}
\frac{\partial P(g)}{\partial \mu_{21}} & =\frac{\lambda_{11}\left(1-a^{k}\right)\left(1-b a^{k-1}\right)-\left(\lambda_{11} \mu_{21}-\lambda_{21} \mu_{11}\right)\left(1-a^{k}\right) a^{k-1}}{k(1-a)^{2}\left(1-b a^{k-1}\right)^{2}} \\
& =\frac{\left(1-a^{k}\right)\left[\lambda_{11}\left(1-a^{k-1}\right)+\lambda_{21} a^{k-1}(1-a)\right]}{k(1-a)^{2}\left(1-b a^{k-1}\right)^{2}}
\end{aligned}
$$


If $a=0$, the partial differential equation can be simplified and we can easily find $\frac{\partial P(g)}{\partial \mu_{21}}>0$.

If $a^{k-1}>0$, we can easily find $\frac{\partial P(g)}{\partial \mu_{21}}>0$.

If $a^{k-1}<0$, that means $a<0$ and therefore $a^{k-2}>0$. The equation can be expressed as follows

$$
\begin{aligned}
\frac{\partial P(g)}{\partial \mu_{21}} & =\frac{\lambda_{11}\left(1-a^{k}\right)\left(1-b a^{k-1}\right)-\left(\lambda_{11} \mu_{21}-\lambda_{21} \mu_{11}\right)\left(1-a^{k}\right) a^{k-1}}{k(1-a)^{2}\left(1-b a^{k-1}\right)^{2}} \\
& =\frac{\left(1-a^{k}\right)\left[\lambda_{11}\left(1-a^{k-1}\right)+\lambda_{21} a^{k-1}(1-a)\right]}{k(1-a)^{2}\left(1-b a^{k-1}\right)^{2}} \\
& =\frac{\left(1-a^{k}\right)(1-a)\left(\lambda_{11} \cdot \Sigma_{m=0}^{k-2} a^{m}+\lambda_{21} a^{k-1}\right)}{k(1-a)^{2}\left(1-b a^{k-1}\right)^{2}} \\
& =\frac{\left(1-a^{k}\right)(1-a)\left(\lambda_{11} \cdot \Sigma_{m=0}^{k-3} a^{m}+\lambda_{11} a^{k-2}+\lambda_{21} a^{k-1}\right)}{k(1-a)^{2}\left(1-b a^{k-1}\right)^{2}} \\
& =\frac{\left(1-a^{k}\right)(1-a)\left(\lambda_{11} \frac{1-a^{k-2}}{1-a}+\left(\lambda_{11}+\lambda_{21} a\right) a^{k-2}\right)}{k(1-a)^{2}\left(1-b a^{k-1}\right)^{2}} \\
& =\frac{\left(1-a^{k}\right)(1-a)\left[\lambda_{11} \frac{1-a^{k-2}}{1-a}+\left(\lambda_{11}+\lambda_{21}-\mu_{21} \lambda_{11}-\mu_{21} \mu_{11}\right) a^{k-2}\right]}{k(1-a)^{2}\left(1-b a^{k-1}\right)^{2}} \\
& =\frac{\left(1-a^{k}\right)(1-a)\left[\lambda_{11} \frac{1-a^{k-2}}{1-a}+\left(\lambda_{11}\left(1-\lambda_{21}\right)+\lambda_{21}\left(1-\mu_{11}\right)\right) a^{k-2}\right]}{k(1-a)^{2}\left(1-b a^{k-1}\right)^{2}} \\
& >0
\end{aligned}
$$

Note that the derivations are both based on the batch size $k \geq 2$.

\section{Proof of Proposition 3.2:}

First we prove $D_{k+1}<D_{k}$ when $k$ is odd, then the monotonicity follows immediately by selecting $e_{11}>e_{21}$ or $e_{11}<e_{21}$. This proof is carried out by induction.

We first show that $D_{2}<D_{1}$. We have

$$
D_{2}-D_{1}=\frac{1-a^{2}}{2(1-b a)}-\frac{1-a}{1-b}
$$




$$
\begin{aligned}
& =-\frac{(1-a)^{2}(1+b)}{2(1-b a)(1-b)} \\
& <0 .
\end{aligned}
$$

Next we assume that

$$
D_{2 j}<D_{2 j-1} \text {. }
$$

This implies that

$$
\begin{aligned}
D_{2 j}-D_{2 j-1} & =\frac{1-a^{2 j}}{2 j\left(1-b a^{2 j-1}\right)}-\frac{1-a^{2 j-1}}{(2 j-1)\left(1-b a^{2 j-2}\right)} \\
& =\frac{-\left(1-b a^{2 j-2}\right)\left(1-a^{2 j}\right)+(1-a) 2 j a^{2 j-2}(a-b)}{2 j(2 j-1)\left(1-b a^{2 j-1}\right)\left(1-b a^{2 j-2}\right)} \\
& <0 .
\end{aligned}
$$

It follows that

$$
(1-a)(a-b) 2 j a^{2 j-2}<\left(1-a^{2 j}\right)\left(1-b a^{2 j-2}\right),
$$

i.e.,

$$
(1-a)(a-b) 2 j a^{2 j}<a^{2}\left(1-a^{2 j}\right)\left(1-b a^{2 j-2}\right),
$$

Now we need to show that $D_{2 j+2}<D_{2 j+1}$.

$$
\begin{aligned}
& D_{2 j+2}-D_{2 j+1} \\
= & \frac{1-a^{2 j+2}}{(2 j+2)\left(1-b a^{2 j+1}\right)}-\frac{1-a^{2 j+1}}{(2 j+1)\left(1-b a^{2 j}\right)} \\
= & \frac{-\left(1-a^{2 j}\right)\left(1-b a^{2 j+2}\right)-(1-a)^{2}(1+b) a^{2 j}+2 j a^{2 j}(1-a)(a-b)}{(2 j+1)(2 j+2)\left(1-b a^{2 j+1}\right)\left(1-b a^{2 j}\right)} \\
< & \frac{-\left(1-a^{2 j}\right)\left(1-b a^{2 j+2}\right)-(1-a)^{2}(1+b) a^{2 j}+a^{2}\left(1-a^{2 j}\right)\left(1-b a^{2 j-2}\right)}{(2 j+1)(2 j+2)\left(1-b a^{2 j+1}\right)\left(1-b a^{2 j}\right)} \\
= & \frac{-\left(1-a^{2 j}\right)\left(1-b a^{2 j+2}-a^{2}+b a^{2 j}\right)-(1-a)^{2}(1+b) a^{2 j}}{(2 j+1)(2 j+2)\left(1-b a^{2 j+1}\right)\left(1-b a^{2 j}\right)}
\end{aligned}
$$




$$
\begin{aligned}
& =\frac{-\left(1-a^{2 j}\right)\left(1-a^{2}+b a^{2 j}\left(1-a^{2}\right)\right)-(1-a)^{2}(1+b) a^{2 j}}{(2 j+1)(2 j+2)\left(1-b a^{2 j+1}\right)\left(1-b a^{2 j}\right)} \\
& =\frac{-\left(1-a^{2 j}\right)\left(1-a^{2}\right)\left(1-b a^{2 j}\right)-(1-a)^{2}(1+b) a^{2 j}}{(2 j+1)(2 j+2)\left(1-b a^{2 j+1}\right)\left(1-b a^{2 j}\right)} \\
& <0 .
\end{aligned}
$$

By induction, $D_{k+1}<D_{k}$ when $\mathrm{k}$ is odd.

\section{Proof of Proposition 3.3:}

First we prove $D_{k+2}<D_{k}$ when $\mathrm{k}$ is odd, then the monotonicity follows immediately by selecting $e_{11}>e_{21}$ or $e_{11}<e_{21}$.

We first show that $D_{3}<D_{1}$. Then

$$
\begin{aligned}
D_{3}-D_{1} & =\frac{1-a^{3}}{3\left(1-b a^{2}\right)}-\frac{1-a}{1-b} \\
& =\frac{1-a}{3\left(1-b a^{2}\right)(1-b)}\left[\left(1+a+a^{2}\right)(1-b)-3\left(1-b a^{2}\right)\right] \\
& =-\frac{\left.(1-a)^{2}[1+a b+(1+a)(1+a))\right]}{3\left(1-b a^{2}\right)(1-b)} \\
& =<0 .
\end{aligned}
$$

Next we assume that

$$
D_{2 j+1}<D_{2 j-1}
$$

This implies that

$$
\begin{aligned}
D_{2 j+1}-D_{2 j-1} & =\frac{1-a^{2 j+1}}{(2 j+1)\left(1-b a^{2 j}\right)}-\frac{1-a^{2 j-1}}{(2 j-1)\left(1-b a^{2 j-2}\right)} \\
& =\frac{(2 j-1)\left(1-b a^{2 j-2}\right)\left(1-a^{2 j+1}\right)-(2 j+1)\left(1-b a^{2 j}\right)\left(1-a^{2 j-1}\right)}{(2 j-1)(2 j+1)\left(1-b a^{2 j-2}\right)\left(1-b a^{2 j}\right)} \\
& =\frac{-2\left(1-a^{2 j-1}\right)\left(1-b a^{2 j}\right)+(2 j-1) a^{2 j-2}\left(1-a^{2}\right)(a-b)}{(2 j-1)(2 j+1)\left(1-b a^{2 j-2}\right)\left(1-b a^{2 j}\right)} \\
& <0 .
\end{aligned}
$$


It follows that

$$
-2\left(1-a^{2 j-1}\right)\left(1-b a^{2 j}\right)+(2 j-1) a^{2 j-2}\left(1-a^{2}\right)(a-b)<0
$$

Now we need to show that $D_{2 j+3}<D_{2 j+1}$.

$$
\begin{aligned}
& D_{2 j+3}-D_{2 j+1}=\frac{1-a^{2 j+3}}{(2 j+3)\left(1-b a^{2 j+2}\right)}-\frac{1-a^{2 j+1}}{(2 j+1)\left(1-b a^{2 j}\right)} \\
& =\frac{(2 j+1)\left(1-b a^{2 j}\right)\left(1-a^{2 j+3}\right)-(2 j+3)\left(1-b a^{2 j+2}\right)\left(1-a^{2 j+1}\right)}{(2 j+3)(2 j+1)\left(1-b a^{2 j+2}\right)\left(1-b a^{2 j}\right)} \\
& =\left[-2\left(1-a^{2 j-1}\right)\left(1-b a^{2 j+4}\right)-2 a^{2 j-1}\left(1-a^{2}\right)^{2}(1+a b)\right. \\
& \left.+(2 j-1) a^{2 j}\left(1-a^{2}\right)(a-b)\right] \\
& /\left[(2 j+3)(2 j+1)\left(1-b a^{2 j+2}\right)\left(1-b a^{2 j}\right)\right] \\
& <\left[-2\left(1-a^{2 j-1}\right)\left(1-b a^{2 j+4}\right)-2 a^{2 j-1}\left(1-a^{2}\right)^{2}(1+a b)\right. \\
& \left.+2 a^{2}\left(1-a^{2 j-1}\right)\left(1-b a^{2 j}\right)\right] \\
& /\left[(2 j+3)(2 j+1)\left(1-b a^{2 j+2}\right)\left(1-b a^{2 j}\right)\right] \\
& =\frac{-2\left(1-a^{2 j-1}\right)\left(1-a^{2}\right)\left(1+b a^{2 j+2}\right)-2 a^{2 j-1}\left(1-a^{2}\right)^{2}(1+a b)}{(2 j+3)(2 j+1)\left(1-b a^{2 j+2}\right)\left(1-b a^{2 j}\right)} \\
& =-2(1-a)^{2} \frac{\left(1-a^{2 j-1}\right)\left(1+b a^{2 j+2}\right)+a^{2 j-1}\left(1-a^{2}\right)(1+a b)}{(2 j+3)(2 j+1)\left(1-b a^{2 j+2}\right)\left(1-b a^{2 j}\right)} \\
& =-2(1-a)^{2} \frac{1-b a^{4 j+1}-a^{2 j+1}+b a^{2 j}}{(2 j+3)(2 j+1)\left(1-b a^{2 j+2}\right)\left(1-b a^{2 j}\right)} \\
& =-2(1-a)^{2} \frac{\left(1-a^{2 j+1}\right)\left(1+b a^{2 j}\right)}{(2 j+3)(2 j+1)\left(1-b a^{2 j+2}\right)\left(1-b a^{2 j}\right)} \\
& <0 \text {. }
\end{aligned}
$$

By induction, $D_{k+2}<D_{k}$ when $\mathrm{k}$ is odd.

Next we prove $D_{k+2}<D_{k}$ when $\mathrm{k}$ is even, then the monotonicity follows immediately by selecting $e_{11}>e_{21}$ or $e_{11}<e_{21}$. 
We first show that $D_{4}<D_{2}$. Then

$$
\begin{aligned}
D_{4}-D_{2} & =\frac{1-a^{4}}{4\left(1-b a^{3}\right)}-\frac{1-a^{2}}{2(1-b a)} \\
& =\frac{1-a^{2}}{4\left(1-b a^{3}\right)(1-b a)}\left[\left(1+a^{2}\right)(1-b a)-2\left(1-b a^{3}\right)\right] \\
& =-\frac{\left(1-a^{2}\right)^{2}(1+b a)}{4\left(1-b a^{3}\right)(1-b a)} \\
& <0 .
\end{aligned}
$$

Next we assume that

$$
D_{2 j+2}<D_{2 j}
$$

This implies that

$$
\begin{aligned}
D_{2 j+2}-D_{2 j} & =\frac{1-a^{2 j+2}}{(2 j+2)\left(1-b a^{2 j+1}\right)}-\frac{1-a^{2 j}}{(2 j)\left(1-b a^{2 j-1}\right)} \\
& =\frac{(2 j)\left(1-b a^{2 j-1}\right)\left(1-a^{2 j+2}\right)-(2 j+2)\left(1-b a^{2 j+1}\right)\left(1-a^{2 j}\right)}{(2 j)(2 j+2)\left(1-b a^{2 j-1}\right)\left(1-b a^{2 j+1}\right)} \\
& =\frac{-2\left(1-a^{2 j}\right)\left(1-b a^{2 j+1}\right)+(2 j) a^{2 j-1}\left(1-a^{2}\right)(a-b)}{(2 j)(2 j+2)\left(1-b a^{2 j-1}\right)\left(1-b a^{2 j+1}\right)} \\
& <0 .
\end{aligned}
$$

It follows that

$$
-2\left(1-a^{2 j}\right)\left(1-b a^{2 j+1}\right)+(2 j) a^{2 j-1}\left(1-a^{2}\right)(a-b)<0 .
$$

Now we need to show that $D_{2 j+4}<D_{2 j+2}$.

$$
\begin{aligned}
D_{2 j+4}-D_{2 j+2}= & \frac{1-a^{2 j+4}}{(2 j+4)\left(1-b a^{2 j+3}\right)}-\frac{1-a^{2 j+2}}{(2 j+2)\left(1-b a^{2 j+1}\right)} \\
= & \frac{(2 j+2)\left(1-b a^{2 j+1}\right)\left(1-a^{2 j+4}\right)-(2 j+4)\left(1-b a^{2 j+3}\right)\left(1-a^{2 j+2}\right)}{(2 j+2)(2 j+4)\left(1-b a^{2 j+1}\right)\left(1-b a^{2 j+3}\right)} \\
= & {\left[-2\left(1-a^{2 j}\right)\left(1-b a^{2 j+5}\right)-2 a^{2 j}\left(1-a^{2}\right)^{2}(1+a b)\right.} \\
& \left.+(2 j) a^{2 j+1}\left(1-a^{2}\right)(a-b)\right]
\end{aligned}
$$




$$
\begin{aligned}
& /\left[(2 j+2)(2 j+4)\left(1-b a^{2 j+1}\right)\left(1-b a^{2 j+3}\right)\right] \\
< & {\left[-2\left(1-a^{2 j}\right)\left(1-b a^{2 j+5}\right)-2 a^{2 j}\left(1-a^{2}\right)^{2}(1+a b)\right.} \\
& \left.+2 a^{2}\left(1-a^{2 j}\right)\left(1-b a^{2 j+1}\right)\right] \\
& /\left[(2 j+2)(2 j+4)\left(1-b a^{2 j+1}\right)\left(1-b a^{2 j+3}\right)\right] \\
= & \frac{-2\left(1-a^{2 j}\right)\left(1-a^{2}\right)\left(1+b a^{2 j+3}\right)-2 a^{2 j}\left(1-a^{2}\right)^{2}(1+a b)}{(2 j+2)(2 j+4)\left(1-b a^{2 j+1}\right)\left(1-b a^{2 j+3}\right)} \\
< & 0 .
\end{aligned}
$$

By induction, $D_{k+2}<D_{k}$ when $\mathrm{k}$ is even.

Therefore, in both cases we have $D_{k+2}-D_{k}<0$. The monotonicity statement holds.

\section{Appendix B: Proofs of Chapter 4}

\section{Proof of Theorem 4.1:}

By substituting good states until only one exists, we have

$$
\begin{aligned}
P\left(g_{11}\right) & =\delta_{12}\left[\delta_{22} P\left(g_{21}\right)+\frac{\mu_{22}}{4}\right]+\frac{\mu_{12}}{4} \\
& =\delta_{12} \delta_{22}\left[\delta_{21} P\left(g_{12}\right)+\frac{\mu_{21}}{4}\right]+\delta_{12} \frac{\mu_{22}}{4}+\frac{\mu_{12}}{4} \\
& =\delta_{12} \delta_{22} \delta_{21}\left[\delta_{11} P\left(g_{11}\right)+\frac{\mu_{11}}{4}\right]+\delta_{12} \delta_{22} \frac{\mu_{21}}{4}+\delta_{12} \frac{\mu_{22}}{4}+\frac{\mu_{12}}{4} \\
& =\delta_{12} \delta_{22} \delta_{21} \delta_{11} P\left(g_{11}\right)+\delta_{12} \delta_{22} \delta_{21} \frac{\mu_{11}}{4}+\delta_{12} \delta_{22} \frac{\mu_{21}}{4}+\delta_{12} \frac{\mu_{22}}{4}+\frac{\mu_{12}}{4} .
\end{aligned}
$$

Therefore,

$$
P\left(g_{11}\right)=\frac{\delta_{12} \delta_{22} \delta_{21} \frac{\mu_{11}}{4}+\delta_{12} \delta_{22} \frac{\mu_{21}}{4}+\delta_{12} \frac{\mu_{22}}{4}+\frac{\mu_{12}}{4}}{1-\delta_{12} \delta_{22} \delta_{21} \delta_{11}} .
$$


Similarly,

$$
\begin{aligned}
& P\left(g_{12}\right)=\frac{\delta_{11} \delta_{12} \delta_{22} \frac{\mu_{21}}{4}+\delta_{11} \delta_{12} \frac{\mu_{22}}{4}+\delta_{11} \frac{\mu_{12}}{4}+\frac{\mu_{11}}{4}}{1-\delta_{12} \delta_{22} \delta_{21} \delta_{11}}, \\
& P\left(g_{21}\right)=\frac{\delta_{21} \delta_{11} \delta_{12} \frac{\mu_{22}}{4}+\delta_{21} \delta_{11} \frac{\mu_{12}}{4}+\delta_{21} \frac{\mu_{11}}{4}+\frac{\mu_{21}}{4}}{1-\delta_{12} \delta_{22} \delta_{21} \delta_{11}}, \\
& P\left(g_{22}\right)=\frac{\delta_{22} \delta_{21} \delta_{11} \frac{\mu_{12}}{4}+\delta_{22} \delta_{21} \frac{\mu_{11}}{4}+\delta_{22} \frac{\mu_{21}}{4}+\frac{\mu_{22}}{4}}{1-\delta_{12} \delta_{22} \delta_{21} \delta_{11}} .
\end{aligned}
$$

The total good part probability can be derived

$$
\begin{aligned}
P\left(g_{b t}\right)= & \frac{1}{4} \cdot \frac{\left(1+\delta_{21}+\delta_{22} \delta_{21}+\delta_{12} \delta_{22} \delta_{21}\right) \mu_{11}}{1-\delta_{11} \delta_{12} \delta_{22} \delta_{21}} \\
& +\frac{1}{4} \cdot \frac{\left(1+\delta_{11}+\delta_{21} \delta_{11}+\delta_{22} \delta_{21} \delta_{11}\right) \mu_{12}}{1-\delta_{11} \delta_{12} \delta_{22} \delta_{21}} \\
& +\frac{1}{4} \cdot \frac{\left(1+\delta_{22}+\delta_{12} \delta_{22}+\delta_{11} \delta_{12} \delta_{22}\right) \mu_{21}}{1-\delta_{11} \delta_{12} \delta_{22} \delta_{21}} \\
& +\frac{1}{4} \cdot \frac{\left(1+\delta_{12}+\delta_{11} \delta_{12}+\delta_{21} \delta_{11} \delta_{12}\right) \mu_{22}}{1-\delta_{11} \delta_{12} \delta_{22} \delta_{21}} .
\end{aligned}
$$

\section{Proof of Proposition 4.2;}

When $n=2$, we have

$$
\begin{aligned}
\mu_{\text {no-switch }}^{\text {total }} & =\mu_{11}+\mu_{22}, \\
\mu_{\text {switch }}^{\text {total }} & =\mu_{12}+\mu_{21} .
\end{aligned}
$$

First, we prove

$$
\begin{aligned}
\frac{\mu_{\text {switch }}^{\text {total }}}{2\left(1+\delta_{\max }\right)} & <P\left(g_{s s}\right)<\frac{\mu_{\text {switch }}^{\text {total }}}{2\left(1-\delta_{\max }\right)} \\
\frac{\mu_{\text {no-switch }}^{\text {total }}+\mu_{\text {switch }}^{\text {total }}}{4\left(1+\delta_{\max }\right)} & <P\left(g_{\text {bt }}\right)<\frac{\mu_{\text {no-switch }}^{\text {total }}+\mu_{\text {switch }}^{\text {total }}}{4\left(1-\delta_{\max }\right)} .
\end{aligned}
$$

For strictly sequencing policy, we have

$$
\begin{aligned}
& P\left(g_{11}\right)=\delta_{12} P\left(g_{21}\right)+\frac{1}{2} \mu_{12}, \\
& P\left(g_{21}\right)=\delta_{21} P\left(g_{11}\right)+\frac{1}{2} \mu_{21} .
\end{aligned}
$$


Since $-\delta_{\max }<\delta_{12}, \delta_{21}<\delta_{\max }$, we can obtain

$$
\begin{aligned}
& -\delta_{\max } P\left(g_{21}\right)+\frac{1}{2} \mu_{12}<P\left(g_{11}\right)<\delta_{\max } P\left(g_{21}\right)+\frac{1}{2} \mu_{12} \\
& -\delta_{\max } P\left(g_{11}\right)+\frac{1}{2} \mu_{21}<P\left(g_{21}\right)<\delta_{\max } P\left(g_{11}\right)+\frac{1}{2} \mu_{21}
\end{aligned}
$$

Summing up these two equations, we have

$$
-\delta_{\text {max }} P\left(g_{s s}\right)+\frac{1}{2} \mu_{\text {switch }}^{\text {total }}<P\left(g_{s s}\right)<\delta_{\text {max }} P\left(g_{s s}\right)+\frac{1}{2} \mu_{\text {switch }}^{\text {total }}
$$

Therefore,

$$
\frac{\mu_{\text {switch }}^{\text {total }}}{2\left(1+\delta_{\max }\right)}<P\left(g_{s s}\right)<\frac{\mu_{\text {switch }}^{\text {total }}}{2\left(1-\delta_{\max }\right)}
$$

For batch policy, we have

$$
\begin{aligned}
& P\left(g_{11}\right)=\delta_{12} P\left(g_{22}\right)+\frac{1}{4} \mu_{12}, \\
& P\left(g_{12}\right)=\delta_{11} P\left(g_{11}\right)+\frac{1}{4} \mu_{11}, \\
& P\left(g_{21}\right)=\delta_{21} P\left(g_{12}\right)+\frac{1}{4} \mu_{21}, \\
& P\left(g_{22}\right)=\delta_{22} P\left(g_{21}\right)+\frac{1}{4} \mu_{22} .
\end{aligned}
$$

Since $-\delta_{\max }<\delta_{12}, \delta_{11}, \delta_{21}, \delta_{22}<\delta_{\max }$, we can obtain

$$
\begin{aligned}
& -\delta_{\text {max }} P\left(g_{22}\right)+\frac{1}{4} \mu_{12}<P\left(g_{11}\right)<\delta_{\text {max }} P\left(g_{22}\right)+\frac{1}{4} \mu_{12}, \\
& -\delta_{\text {max }} P\left(g_{11}\right)+\frac{1}{4} \mu_{11}<P\left(g_{12}\right)<\delta_{\text {max }} P\left(g_{11}\right)+\frac{1}{4} \mu_{11} \\
& -\delta_{\max } P\left(g_{12}\right)+\frac{1}{4} \mu_{21}<P\left(g_{21}\right)<\delta_{\max } P\left(g_{12}\right)+\frac{1}{4} \mu_{21} \\
& -\delta_{\max } P\left(g_{21}\right)+\frac{1}{4} \mu_{22}<P\left(g_{22}\right)<\delta_{\max } P\left(g_{21}\right)+\frac{1}{4} \mu_{22} .
\end{aligned}
$$

Similarly, we can finally obtain

$$
\frac{\mu_{\text {switch }}^{\text {total }}+\mu_{\text {no-switch }}^{\text {total }}}{4\left(1+\delta_{\max }\right)}<P\left(g_{b t}\right)<\frac{\mu_{\text {switch }}^{\text {total }}+\mu_{\text {no-switch }}^{\text {total }}}{4\left(1-\delta_{\max }\right)}
$$


Next, we proceed to prove the proposition.

$$
\begin{aligned}
& \text { If } \mu_{\text {no-switch }}^{\text {total }}>\mu_{\text {switch }}^{\text {total }} \text { and } \delta_{\max }<\frac{\mu_{\text {no-switch }}^{\text {total }}-\mu_{\text {switch }}^{\text {total }}}{\mu_{\text {no-switch }}^{\text {total }}+3 \mu_{\text {switch }}^{\text {total }}} \text {, then } \\
& \qquad \frac{\mu_{\text {switch }}^{\text {total }}+\mu_{\text {no-switch }}^{\text {total }}}{4\left(1+\delta_{\max }\right)}>\frac{\mu_{\text {switch }}^{\text {total }}}{2\left(1-\delta_{\max }\right)} .
\end{aligned}
$$

Therefore,

$$
P\left(g_{b t}\right)>P\left(g_{s s}\right)
$$

If $\mu_{\text {no-switch }}^{\text {total }}<\mu_{\text {switch }}^{\text {total }}$ and $\delta_{\text {max }}<\frac{\mu_{\text {switch }}^{\text {total }}-\mu_{\text {no-switch }}^{\text {total }}}{\mu_{\text {no-switch }}^{\text {total }}+3 \mu_{\text {switch }}^{\text {total }}}$, then

$$
\frac{\mu_{\text {switch }}^{\text {total }}+\mu_{\text {no-switch }}^{\text {total }}}{4\left(1-\delta_{\max }\right)}<\frac{\mu_{\text {switch }}^{\text {total }}}{2\left(1+\delta_{\max }\right)}
$$

Therefore,

$$
P\left(g_{b t}\right)<P\left(g_{s s}\right)
$$

\section{Proof of Equation 4.35:}

First, we prove that $\operatorname{det}(I-\Gamma)=1-\prod_{i=1}^{K} \gamma_{i}$.

$$
\begin{aligned}
\operatorname{det}(I-\Gamma)= & \operatorname{det}\left(\begin{array}{ccccc}
1 & 0 & \cdots & 0 & -\gamma_{1} \\
-\gamma_{2} & 1 & \cdots & 0 & 0 \\
0 & -\gamma_{3} & \cdots & 0 & 0 \\
\vdots & \vdots & \vdots & \vdots & \vdots \\
0 & 0 & \cdots & -\gamma_{K} & 1
\end{array}\right) \\
= & \left(\begin{array}{cccc}
1 & \cdots & 0 & 0 \\
-\gamma_{3} & \cdots & 0 & 0 \\
\vdots & \vdots & \vdots & \vdots \\
0 & \cdots & -\gamma_{K} & 1
\end{array}\right)+(-1)^{K+1}\left(-\gamma_{1}\right)\left(\begin{array}{cccc}
-\gamma_{2} & 1 & \cdots & 0 \\
0 & -\gamma_{3} & \cdots & 0 \\
\vdots & \vdots & \vdots & \vdots \\
0 & 0 & \cdots & -\gamma_{K}
\end{array}\right) \\
= & 1+(-1)^{K+1}\left(-\gamma_{1}\right) \Pi_{i=2}^{K}\left(-\gamma_{i}\right) \\
= & 1-\prod_{i=1}^{K} \gamma_{i}
\end{aligned}
$$


Next, we show that $\Gamma^{K}=\Pi_{i=1}^{K} \gamma_{i} I$. Define transformation matrix $I_{r}$ and a serial of diagonal matrix $\Gamma_{i}, i=1, \ldots, K$, as

$$
\begin{aligned}
I_{r}= & \left(\begin{array}{ccccc}
0 & 0 & \cdots & 0 & 1 \\
1 & 0 & \cdots & 0 & 0 \\
0 & 1 & \cdots & 0 & 0 \\
\vdots & \vdots & \vdots & \vdots & \vdots \\
0 & 0 & \cdots & 1 & 0
\end{array}\right), \\
\Gamma_{i}= & \left(\begin{array}{cccccc}
\gamma_{i} & 0 & \cdots & 0 & \cdots & 0 \\
0 & \gamma_{i+1} & \cdots & 0 & \cdots & 0 \\
\vdots & \vdots & \vdots & \vdots & \vdots & \vdots \\
0 & 0 & \cdots & \gamma_{1} & \cdots & 0 \\
\vdots & \vdots & \vdots & \vdots & \vdots & \vdots \\
0 & 0 & \cdots & 0 & \cdots & \gamma_{i-1}
\end{array}\right) .
\end{aligned}
$$

The following properties are obtained:

$$
\begin{aligned}
I_{r}^{K} & =I, \\
\Gamma & =\Gamma_{1} I_{r}, \\
I_{r}^{-1} \Gamma_{i} I_{r} & =\Gamma_{i+1}, \\
\prod_{i=1}^{K} \Gamma_{i} & =\prod_{i=1}^{K} \gamma_{i} I .
\end{aligned}
$$

It follows that:

$$
\begin{aligned}
\Gamma^{K} & =\Gamma_{1} I_{r} \Gamma_{1} I_{r} \cdots \Gamma_{1} I_{r} \Gamma_{1} I_{r} \Gamma_{1} I_{r} \\
& =\Gamma_{1} I_{r} \Gamma_{1} I_{r} \cdots \Gamma_{1} I_{r} \Gamma_{1} I_{r} I_{r} I_{r}^{-1} \Gamma_{1} I_{r} \\
& =\Gamma_{1} I_{r} \Gamma_{1} I_{r} \cdots \Gamma_{1} I_{r} \Gamma_{1} I_{r} I_{r} \Gamma_{2} \\
& =\Gamma_{1} I_{r} \Gamma_{1} I_{r} \cdots \Gamma_{1} I_{r} I_{r} I_{r}^{-1} \Gamma_{1} I_{r} I_{r} \Gamma_{2} \\
& =\Gamma_{1} I_{r} \Gamma_{1} I_{r} \cdots \Gamma_{1} I_{r}^{2} \Gamma_{2} I_{r} \Gamma_{2} \\
& =\Gamma_{1} I_{r} \Gamma_{1} I_{r} \cdots \Gamma_{1} I_{r}^{2} I_{r} I_{r}^{-1} \Gamma_{2} I_{r} \Gamma_{2} \\
& =\Gamma_{1} I_{r} \Gamma_{1} I_{r} \cdots \Gamma_{1} I_{r}^{3} \Gamma_{3} \Gamma_{2}
\end{aligned}
$$




$$
\begin{aligned}
& =\Gamma_{1} I_{r} \Gamma_{1} I_{r} \cdots \Gamma_{1} I_{r}^{i} \Gamma_{i} \Gamma_{i-1} \cdots \Gamma_{2} \\
& =\Gamma_{1} I_{r}^{K} \Pi_{i=2}^{K} \Gamma_{i} \\
& =\prod_{i=1}^{K} \Gamma_{i} \\
& =\prod_{i=1}^{K} \gamma_{i} I
\end{aligned}
$$

Finally,we obtain

$$
\begin{aligned}
(I-\Gamma) \frac{\sum_{i=1}^{K} \Gamma^{i-1}}{\operatorname{det}(I-\Gamma)} & =\frac{\sum_{i=1}^{K} \Gamma^{i-1}}{\operatorname{det}(I-\Gamma)}(I-\Gamma) \\
& =\frac{\sum_{i=1}^{K} \Gamma^{i-1}-\sum_{i=1}^{K} \Gamma^{i}}{\operatorname{det}(I-\Gamma)} \\
& =\frac{I-\Gamma^{K}}{\operatorname{det}(I-\Gamma)} \\
& =\frac{I-\prod_{i=1}^{K} \gamma_{i} I}{\operatorname{det}(I-\Gamma)} \\
& =\frac{\left(1-\Pi_{i=1}^{K} \gamma_{i}\right) I}{1-\prod_{i=1}^{K} \gamma_{i}} \\
& =I
\end{aligned}
$$

Therefore,

$$
(I-\Gamma)^{-1}=\frac{\sum_{i=1}^{K} \Gamma^{i-1}}{\operatorname{det}(I-\Gamma)}
$$

Equation 4.35 follows immediately.

\section{Proof of Proposition 4.5:}

For batch policy, we have

$$
\begin{aligned}
P\left(g_{b t}^{l}\right)-P\left(g_{b t}^{m}\right)= & \frac{\mu_{s_{1}^{l}, s_{n}^{l}}+\sum_{i=2}^{n} \mu_{s_{i}^{l}, s_{i-1}^{l}}+\sum_{i=1}^{n}\left(k_{s_{i}^{l}}-1\right) \mu_{s_{i}^{l}, s_{i}^{l}}}{\sum_{i=1}^{n} k_{s_{i}^{l}}} \\
& -\frac{\mu_{s_{1}^{m}, s_{n}^{m}}+\sum_{i=2}^{n} \mu_{s_{i}^{m}, s_{i-1}^{m}}+\sum_{i=1}^{n}\left(k_{s_{i}^{m}}-1\right) \mu_{s_{i}^{m}, s_{i}^{m}}}{\sum_{i=1}^{n} k_{s_{i}^{m}}} .
\end{aligned}
$$


Note that $\sum_{i=1}^{n} k_{s_{i}^{l}}=\sum_{i=1}^{n} k_{s_{i}^{m}}$ and $\sum_{i=1}^{n}\left(k_{s_{i}^{l}}-1\right) \mu_{s_{i}^{l}, s_{i}^{l}}=\sum_{i=1}^{n}\left(k_{s_{i}^{m}}-1\right) \mu_{s_{i}^{m}, s_{i}^{m}}$, where $s_{i}^{l}, s_{i}^{m} \in\{1,2, \ldots, n\}$. Then, we have

$$
P\left(g_{b t}^{l}\right)-P\left(g_{b t}^{m}\right)=\frac{\left(\mu_{s_{1}^{l}, s_{n}^{l}}+\sum_{i=2}^{n} \mu_{s_{i}^{l}, s_{i-1}^{l}}\right)-\left(\mu_{s_{1}^{m}, s_{n}^{m}}+\sum_{i=2}^{n} \mu_{s_{i}^{m}, s_{i-1}^{m}}\right)}{\sum_{i=1}^{n} k_{s_{i}^{l}}} .
$$

For strictly sequencing policy, we obtain

$$
\begin{aligned}
P\left(g_{s s}^{l}\right)-P\left(g_{s s}^{m}\right) & =\frac{\mu_{s_{1}^{l}, s_{n}^{l}}+\sum_{i=2}^{n} \mu_{s_{i}^{l}, s_{i-1}^{l}}}{n}-\frac{\mu_{s_{1}^{m}, s_{n}^{m}}+\sum_{i=2}^{n} \mu_{s_{i}^{m}, s_{i-1}^{m}}}{n} \\
& =\frac{\left(\mu_{s_{1}^{l}, s_{n}^{l}}+\sum_{i=2}^{n} \mu_{s_{i}^{l}, s_{i-1}^{l}}\right)-\left(\mu_{s_{1}^{m}, s_{n}^{m}}+\sum_{i=2}^{n} \mu_{s_{i}^{m}, s_{i-1}^{m}}\right)}{n} .
\end{aligned}
$$

Thus, the numerators are the same in both comparisons, which implies that

$$
P\left(g_{b t}^{l}\right)>P\left(g_{b t}^{m}\right) \Longleftrightarrow P\left(g_{s s}^{l}\right)>P\left(g_{s s}^{m}\right)
$$

\section{Proof of Proposition 4.7:}

For a given sequence $s^{l}$, we have

$$
\begin{aligned}
P\left(g_{b t}^{l}\right)-P\left(g_{s s}^{l}\right)= & \frac{\mu_{s_{1}^{l}, s_{n}^{l}}+\sum_{i=2}^{n} \mu_{s_{i}^{l}, s_{i-1}^{l}}+\sum_{i=1}^{n}\left(k_{s_{i}^{l}}-1\right) \mu_{s_{i}^{l}, s_{i}^{l}}}{\sum_{i=1}^{n} k_{s_{i}^{l}}}-\frac{\mu_{s_{1}^{l}, s_{n}^{l}}+\sum_{i=2}^{n} \mu_{s_{i}^{l}, s_{i-1}^{l}}}{n} \\
= & \frac{\sum_{i=1}^{n}\left(k_{s_{i}^{l}}-1\right) \mu_{s_{i}^{l}, s_{i}^{l}}}{\sum_{i=1}^{n} k_{s_{i}^{l}}}-\left(\frac{1}{n}-\frac{1}{\sum_{i=1}^{n} k_{s_{i}^{l}}}\right)\left(\mu_{s_{1}^{l}, s_{n}^{l}}+\sum_{i=2}^{n} \mu_{s_{i}^{l}, s_{i-1}^{l}}\right) \\
= & \sum_{i=1}^{n}\left[\frac{k_{s_{i}^{l}}}{\sum_{i=1}^{n} k_{s_{i}^{l}}}-\frac{1}{\sum_{i=1}^{n} k_{s_{i}^{l}}^{l}}\right] \mu_{s_{i}^{l}, s_{i}^{l}} \\
& -\left(\frac{1}{n}-\frac{1}{\sum_{i=1}^{n} k_{s_{i}^{l}}}\right)\left(\mu_{s_{1}^{l}, s_{n}^{l}}+\sum_{i=2}^{n} \mu_{s_{i}^{l}, s_{i-1}^{l}}\right) \\
= & \widetilde{e}_{\text {no-switch }}^{\text {total }}-\widetilde{e}_{\text {switch }}^{\text {total }} .
\end{aligned}
$$

Therefore,we obtain

$$
P\left(g_{b t}^{l}\right)>P\left(g_{s s}^{l}\right) \Longleftrightarrow \widetilde{e}_{n o-s w i t c h}^{\text {total }}>\widetilde{e}_{\text {switch }}^{\text {total }}
$$




\section{Proof of Proposition 4.8:}

Define function $\operatorname{sum}(\cdot)$ to represent the summation of elements of a vector, i.e.,

$$
\operatorname{sum}(\rho)=\sum_{i=1}^{n} \rho_{i} \text {. }
$$

where $\rho=\left[\rho_{1}, \rho_{2}, \ldots, \rho_{n}\right]^{T}$.

For both policies, the probability of good parts is calculated by

$$
P\left(g^{l}\right)=\sum_{i=1}^{K} x_{i}=\operatorname{sum}\left(\frac{1}{K} \frac{\sum_{i=1}^{K} \Gamma^{i-1}}{\operatorname{det}(I-\Gamma)} \Phi\right) .
$$

In Bernoulli-like case, $\Gamma$ is a matrix with all zero elements. Then, we have

$$
\widetilde{\mu}^{l}=\frac{1}{K} \Phi
$$

In Bernoulli-relax case, $\delta_{\max }=\max _{i j}\left|\delta_{i j}\right|$,

$$
\begin{aligned}
P\left(g^{l}\right)-\frac{1}{1+\delta_{\max }} \widetilde{\mu}^{l} & =\operatorname{sum}\left(\frac{1}{K} \frac{\sum_{i=1}^{K} \Gamma^{i-1}}{\operatorname{det}(I-\Gamma)} \Phi\right)-\operatorname{sum}\left(\frac{1}{K\left(1+\delta_{\max }\right)} \Phi\right) \\
& =\operatorname{sum}\left(\frac{1}{K} \frac{\sum_{i=1}^{K} \Gamma^{i-1}}{\operatorname{det}(I-\Gamma)} \Phi-\frac{1}{K\left(1+\delta_{\max }\right)} \Phi\right) \\
& =\operatorname{sum}\left(\frac{\sum_{i=1}^{K} \Gamma^{i-1} \Phi\left(1+\delta_{\max }\right)-\operatorname{det}(I-\Gamma) \Phi}{K \operatorname{det}(I-\Gamma)\left(1+\delta_{\max }\right)}\right) \\
& =\operatorname{sum}\left(\frac{\sum_{i=1}^{K} \Gamma^{i-1} \Phi\left(1+\delta_{\max }\right)-\left(1-\operatorname{det}\left(\Gamma^{K}\right)\right) \Phi}{K \operatorname{det}(I-\Gamma)\left(1+\delta_{\max }\right)}\right) \\
& =\operatorname{sum}\left(\frac{\Gamma \sum_{i=1}^{K} \Gamma^{i-1} \Phi+\delta_{\max } \sum_{i=1}^{K} \Gamma^{i-1} \Phi}{K \operatorname{det}(I-\Gamma)\left(1+\delta_{\max }\right)}\right) \\
& =\operatorname{sum}\left(\frac{\left(\Gamma+\delta_{\max } I\right) \sum_{i=1}^{K} \Gamma^{i-1} \Phi}{K \operatorname{det}(I-\Gamma)\left(1+\delta_{\max }\right)}\right) \\
& =\operatorname{sum}\left(\frac{\left(\Gamma+\delta_{\max } I\right) X}{1+\delta_{\max }}\right)
\end{aligned}
$$

Denote the transformation matrix $I_{t}$ as

$$
I_{t}=\left(\begin{array}{cccccc}
0 & 1 & 0 & 0 & 0 & 0 \\
0 & 0 & 1 & 0 & 0 & 0 \\
0 & 0 & 0 & \ddots & 0 & 0 \\
0 & 0 & 0 & 0 & 1 & 0 \\
0 & 0 & 0 & 0 & 0 & 1 \\
1 & 0 & 0 & 0 & 0 & 0
\end{array}\right) .
$$


Then,

$$
\operatorname{sum}\left(\frac{\left(\Gamma+\delta_{\max } I\right) X}{1+\delta_{\max }}\right)=\operatorname{sum}\left(\frac{\left(I_{t} \Gamma+\delta_{\max } I\right) X}{1+\delta_{\max }}\right)
$$

Note that matrix $I_{t} \Gamma+\delta_{\max } I$ is diagonal and its elements are non-negative. Therefore,

$$
P\left(g^{l}\right) \geq \frac{1}{1+\delta_{\max }} \widetilde{\mu}^{l}
$$

Similarly, we obtain

$$
\begin{aligned}
& \frac{1}{1-\delta_{\max }} \widetilde{\mu}^{l}-P\left(g^{l}\right)=\operatorname{sum}\left(\frac{1}{K\left(1-\delta_{\max }\right)} \Phi\right)-\operatorname{sum}\left(\frac{1}{K} \frac{\sum_{i=1}^{K} \Gamma^{i-1}}{\operatorname{det}(I-\Gamma)} \Phi\right) \\
& =\operatorname{sum}\left(\frac{1}{K\left(1-\delta_{\max }\right)} \Phi-\frac{1}{K} \frac{\sum_{i=1}^{K} \Gamma^{i-1}}{\operatorname{det}(I-\Gamma)} \Phi\right) \\
& =\operatorname{sum}\left(\frac{\operatorname{det}(I-\Gamma) \Phi-\sum_{i=1}^{K} \Gamma^{i-1} \Phi\left(1-\delta_{\max }\right)}{K \operatorname{det}(I-\Gamma)\left(1-\delta_{\max }\right)}\right) \\
& =\operatorname{sum}\left(\frac{\left(1-\operatorname{det}\left(\Gamma^{K}\right)\right) \Phi-\sum_{i=1}^{K} \Gamma^{i-1} \Phi\left(1-\delta_{\max }\right)}{K \operatorname{det}(I-\Gamma)\left(1-\delta_{\max }\right)}\right) \\
& =\operatorname{sum}\left(\frac{-\Gamma \sum_{i=1}^{K} \Gamma^{i-1} \Phi+\delta_{\max } \sum_{i=1}^{K} \Gamma^{i-1} \Phi}{K \operatorname{det}(I-\Gamma)\left(1-\delta_{\max }\right)}\right) \\
& =\operatorname{sum}\left(\frac{\left(\delta_{\max } I-\Gamma\right) \sum_{i=1}^{K} \Gamma^{i-1} \Phi}{K \operatorname{det}(I-\Gamma)\left(1-\delta_{\max }\right)}\right) \\
& =\operatorname{sum}\left(\frac{\left(\delta_{\max } I-\Gamma\right) X}{1-\delta_{\max }}\right)
\end{aligned}
$$

It follows that

$$
\operatorname{sum}\left(\frac{\left(\delta_{\max } I-\Gamma\right) X}{1-\delta_{\max }}\right)=\operatorname{sum}\left(\frac{\left(\delta_{\max } I-I_{t} \Gamma\right) X}{1-\delta_{\max }}\right)
$$

Matrix $\delta_{\max } I-I_{t} \Gamma$ is again diagonal and its elements are non-negative. Therefore,

$$
P\left(g^{l}\right) \leq \frac{1}{1-\delta_{\max }} \widetilde{\mu}^{l}
$$

Finally, we obtain

$$
\frac{1}{1+\delta_{\max }} \widetilde{\mu}^{l} \leq P\left(g^{l}\right) \leq \frac{1}{1-\delta_{\max }} \widetilde{\mu}^{l}
$$




\section{Proof of Proposition 4.9:}

First, we show that

$$
\frac{\widetilde{\mu}_{b t}^{l}-\widetilde{\mu}_{b t}^{m}}{\widetilde{\mu}_{b t}^{l}+\widetilde{\mu}_{b t}^{m}}=\frac{\widetilde{\mu}_{s s}^{l}-\widetilde{\mu}_{s s}^{m}}{\widetilde{\mu}_{s s}^{l}+\widetilde{\mu}_{s s}^{m}}
$$

Under the Bernoulli-like assumption

$$
\begin{aligned}
\tilde{\mu}_{b t}^{l} & =\frac{\sum_{i=1}^{n}\left(k_{s_{i}^{l}}-1\right) \mu_{s_{i}^{l}, s_{i}^{l}}+\sum_{i=2}^{n} \mu_{s_{i}^{l}, s_{i-1}^{l}}+\mu_{s_{1}^{l}, s_{n}^{l}}}{\sum_{i=1}^{n} k_{s_{i}^{l}}} \\
\tilde{\mu}_{s s}^{l} & =\frac{\sum_{i=2}^{n} \mu_{s_{i}^{l}, s_{i-1}^{l}}+\mu_{s_{1}^{l}, s_{n}^{l}}}{n} .
\end{aligned}
$$

Since the total number of parts are identical for all sequences, i.e., $\sum_{i=1}^{n} k_{s_{i}^{l}}=$ $\sum_{i=1}^{n} k_{s_{i}^{m}}$ and $\sum_{i=1}^{n}\left(k_{s_{i}^{l}}-1\right) \mu_{s_{i}^{l}, s_{i}^{l}}=\sum_{i=1}^{n}\left(k_{s_{i}^{m}}-1\right) \mu_{s_{i}^{m}, s_{i}^{m}}$, where $s_{i}^{l}, s_{i}^{m} \in\{1,2, \ldots, n\}$, we have

$$
\begin{aligned}
& \frac{\widetilde{\mu}_{b t}^{l}-\widetilde{\mu}_{b t}^{m}}{\widetilde{\mu}_{b t}^{l}+\widetilde{\mu}_{b t}^{m}}=\left[\frac{\mu_{s_{1}^{l}, s_{n}^{l}}+\sum_{i=2}^{n} \mu_{s_{i}^{l}, s_{i-1}^{l}}+\sum_{i=1}^{n}\left(k_{s_{i}^{l}}-1\right) \mu_{s_{i}^{l}, s_{i}^{l}}}{\sum_{i=1}^{n} k_{s_{i}^{l}}}\right. \\
& \left.-\frac{\mu_{s_{1}^{m}, s_{n}^{m}}+\sum_{i=2}^{n} \mu_{s_{i}^{m}, s_{i-1}^{m}}+\sum_{i=1}^{n}\left(k_{s_{i}^{m}}-1\right) \mu_{s_{i}^{m}, s_{i}^{m}}}{\sum_{i=1}^{n} k_{s_{i}^{m}}}\right] \\
& /\left[\frac{\mu_{s_{1}^{l}, s_{n}^{l}}+\sum_{i=2}^{n} \mu_{s_{i}^{l}, s_{i-1}^{l}}+\sum_{i=1}^{n}\left(k_{s_{i}^{l}}-1\right) \mu_{s_{i}^{l}, s_{i}^{l}}}{\sum_{i=1}^{n} k_{s_{i}^{l}}}\right. \\
& \left.+\frac{\mu_{s_{1}^{m}, s_{n}^{m}}+\sum_{i=2}^{n} \mu_{s_{i}^{m}, s_{i-1}^{m}}+\sum_{i=1}^{n}\left(k_{s_{i}^{m}}-1\right) \mu_{s_{i}^{m}, s_{i}^{m}}}{\sum_{i=1}^{n} k_{s_{i}^{m}}}\right] \\
& =\frac{\left(\mu_{s_{1}^{l}, s_{n}^{l}}+\sum_{i=2}^{n} \mu_{s_{i}^{l}, s_{i-1}^{l}}\right)-\left(\mu_{s_{1}^{m}, s_{n}^{m}}+\sum_{i=2}^{n} \mu_{s_{i}^{m}, s_{i-1}^{m}}\right)}{\left(\mu_{s_{1}^{l}, s_{n}^{l}}+\sum_{i=2}^{n} \mu_{s_{i}^{l}, s_{i-1}^{l}}\right)+\left(\mu_{s_{1}^{m}, s_{n}^{m}}+\sum_{i=2}^{n} \mu_{s_{i}^{m}, s_{i-1}^{m}}\right)}
\end{aligned}
$$

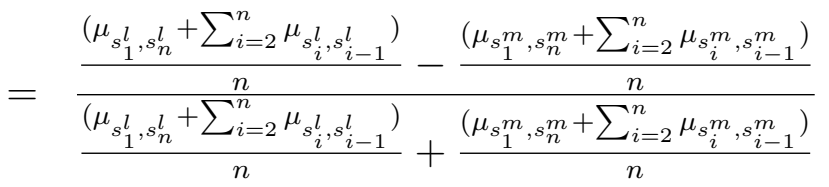

$$
\begin{aligned}
& =\frac{\widetilde{\mu}_{s s}^{l}-\widetilde{\mu}_{s s}^{m}}{\widetilde{\mu}_{s s}^{l}+\widetilde{\mu}_{s s}^{m}} .
\end{aligned}
$$

Next, from Proposition 4.8, we obtain

$$
\frac{\left(\widetilde{\mu}_{b t}^{l}-\widetilde{\mu}_{b t}^{m}\right)-\delta_{\max }\left(\widetilde{\mu}_{b t}^{l}+\widetilde{\mu}_{b t}^{m}\right)}{1-\delta_{\max }^{2}}<P\left(g_{b t}^{l}\right)-P\left(g_{b t}^{m}\right)<\frac{\left(\widetilde{\mu}_{b t}^{l}-\widetilde{\mu}_{b t}^{m}\right)+\delta_{\max }\left(\widetilde{\mu}_{b t}^{l}+\widetilde{\mu}_{b t}^{m}\right)}{1-\delta_{\max }^{2}}
$$




$$
\frac{\left(\widetilde{\mu}_{s s}^{l}-\widetilde{\mu}_{s s}^{m}\right)-\delta_{\max }\left(\widetilde{\mu}_{s s}^{l}+\widetilde{\mu}_{s s}^{m}\right)}{1-\delta_{\max }^{2}}<P\left(g_{s s}^{l}\right)-P\left(g_{s s}^{m}\right)<\frac{\left(\widetilde{\mu}_{s s}^{l}-\widetilde{\mu}_{s s}^{m}\right)+\delta_{\max }\left(\widetilde{\mu}_{s s}^{l}+\widetilde{\mu}_{s s}^{m}\right)}{1-\delta_{\max }^{2}} .
$$

If $\delta_{\max }<\left(\widetilde{\mu}_{b t}^{l}-\widetilde{\mu}_{b t}^{m}\right) /\left(\widetilde{\mu}_{b t}^{l}+\widetilde{\mu}_{b t}^{m}\right)=\left(\widetilde{\mu}_{s s}^{l}-\widetilde{\mu}_{s s}^{m}\right) /\left(\widetilde{\mu}_{s s}^{l}+\widetilde{\mu}_{s s}^{m}\right)$, then

$$
\begin{aligned}
& \left(\widetilde{\mu}_{b t}^{l}-\widetilde{\mu}_{b t}^{m}\right)-\delta_{\max }\left(\widetilde{\mu}_{b t}^{l}+\widetilde{\mu}_{b t}^{m}\right)>0, \\
& \left(\widetilde{\mu}_{s s}^{l}-\widetilde{\mu}_{s s}^{m}\right)-\delta_{\max }\left(\widetilde{\mu}_{s s}^{l}+\widetilde{\mu}_{s s}^{m}\right)>0 .
\end{aligned}
$$

Therefore,

$$
\begin{aligned}
& P\left(g_{b t}^{l}\right)>P\left(g_{b t}^{m}\right), \\
& P\left(g_{s s}^{l}\right)>P\left(g_{s s}^{m}\right) .
\end{aligned}
$$

The arguments follow immediately.

\section{Proof of Proposition 4.10;}

Similar arguments can be applied.

$$
\begin{aligned}
& \frac{\mu_{n o-\text { switch }}^{\text {total }}-\mu_{\text {switch }}^{\text {total }}}{\mu_{\text {no-switch }}^{\text {total }}+\frac{k+1}{k-1} \mu_{\text {switch }}^{\text {total }}} \\
= & \frac{(k-1) \sum_{i=1}^{n} \mu_{s_{i}^{l}, s_{i}^{l}}-(k-1)\left[\mu_{s_{1}^{l}, s_{n}^{l}}+\sum_{i=2}^{n} \mu_{s_{i}^{l}, s_{-1}^{l}}^{l}\right]}{(k-1) \sum_{i=1}^{n} \mu_{s_{i}^{l}, s_{i}^{l}}+(k+1)\left[\mu_{s_{1}^{l}, s_{n}^{l}}+\sum_{i=2}^{n} \mu_{s_{i}^{l}, s_{i-1}^{l}}^{l}\right]} \\
= & {\left[\frac{\mu_{s_{1}^{l}, s_{n}^{l}}+\sum_{i=2}^{n} \mu_{s_{i}^{l}, s_{i-1}^{l}}+\sum_{i=1}^{n}(k-1) \mu_{s_{i}^{l}, s_{i}^{l}}}{n k}-\frac{\mu_{s_{1}^{l}, s_{n}^{l}}+\sum_{i=2}^{n} \mu_{s_{i}^{l}, s_{i-1}^{l}}^{l}}{n}\right] } \\
= & /\left[\frac{\mu_{s_{1}^{l}, s_{n}^{l}}+\sum_{i=2}^{n} \mu_{s_{i}^{l}, s_{i-1}^{l}}+\sum_{i=1}^{n}(k-1) \mu_{s_{i}^{l}, s_{i}^{l}}}{n k}+\frac{\mu_{s_{1}^{l}, s_{n}^{l}}+\sum_{i=2}^{n} \mu_{s_{i}^{l}, s_{i-1}^{l}}^{l}}{n}\right] \\
= & \frac{\widetilde{\mu}_{b t}^{l}-\widetilde{\mu}_{s s}^{l}}{\widetilde{\mu}_{b t}^{l}+\widetilde{\mu}_{s s}^{l}} .
\end{aligned}
$$

Therefore,

$$
\delta_{\text {max }}<\frac{\mu_{\text {no-switch }}^{\text {total }}-\mu_{\text {switch }}^{\text {total }}}{\mu_{\text {no-switch }}^{\text {tol }}+\frac{k+1}{k-1} \mu_{\text {switch }}^{\text {total }}} \quad \text { implies } \quad \delta_{\max }<\frac{\widetilde{\mu}_{b t}^{l}-\widetilde{\mu}_{s s}^{l}}{\widetilde{\mu}_{b t}^{l}+\widetilde{\mu}_{s s}^{l}}
$$


which leads to

$$
\frac{1}{1+\delta_{\max }} \widetilde{\mu}_{b t}^{l}>\frac{1}{1-\delta_{\max }} \widetilde{\mu}_{s s}^{l} .
$$

From Proposition 4.8, we obtain $P\left(g_{b t}^{l}\right)>P\left(g_{s s}^{l}\right)$.

Similarly,

$$
\begin{aligned}
& \frac{\mu_{\text {switch }}^{\text {total }}-\mu_{\text {no-switch }}^{\text {total }}}{\mu_{\text {no-switch }}^{\text {tat }}+\frac{k+1}{k-1} \mu_{\text {switch }}^{\text {total }}} \\
= & \frac{(k-1)\left[\mu_{s_{1}^{l}, s_{n}^{l}}+\sum_{i=2}^{n} \mu_{s_{i}^{l}, s_{i-1}^{l}}\right]-(k-1) \sum_{i=1}^{n} \mu_{s_{i}^{l}, s_{i}^{l}}}{(k-1) \sum_{i=1}^{n} \mu_{s_{i}^{l}, s_{i}^{l}}+(k+1)\left[\mu_{s_{1}^{l}, s_{n}^{l}}+\sum_{i=2}^{n} \mu_{\left.s_{i}^{l}, s_{i-1}^{l}\right]}\right]} \\
= & {\left[\frac{\mu_{s_{1}^{l}, s_{n}^{l}}+\sum_{i=2}^{n} \mu_{s_{i}^{l}, s_{i-1}^{l}}}{n}-\frac{\mu_{s_{1}^{l}, s_{n}^{l}}+\sum_{i=2}^{n} \mu_{s_{i}^{l}, s_{i-1}^{l}}+\sum_{i=1}^{n}(k-1) \mu_{s_{i}^{l}, s_{i}^{l}}}{n k}\right] } \\
& /\left[\frac{\mu_{s_{1}^{l}, s_{n}^{l}}+\sum_{i=2}^{n} \mu_{s_{i}^{l}, s_{i-1}^{l}}+\sum_{i=1}^{n}(k-1) \mu_{s_{i}^{l}, s_{i}^{l}}}{n k}+\frac{\mu_{s_{1}^{l}, s_{n}^{l}}+\sum_{i=2}^{n} \mu_{s_{i}^{l}, s_{i-1}^{l}}}{n}\right] \\
= & \frac{\widetilde{\mu}_{s s}^{l}-\widetilde{\mu}_{b t}^{l}}{\widetilde{\mu}_{b t}^{l}+\widetilde{\mu}_{s s}^{l} .}
\end{aligned}
$$

Therefore,

$$
\delta_{\text {max }}<\frac{\mu_{\text {switch }}^{\text {total }}-\mu_{\text {no-switch }}^{\text {total }}}{\mu_{\text {no-switch }}^{\text {total }}+\frac{k+1}{k-1} \mu_{\text {switch }}^{\text {total }}} \quad \text { implies } \quad \delta_{\text {max }}<\frac{\widetilde{\mu}_{s s}^{l}-\widetilde{\mu}_{b t}^{l}}{\widetilde{\mu}_{b t}^{l}+\widetilde{\mu}_{s s}^{l}} \text {, }
$$

which leads to

$$
\frac{1}{1+\delta_{\max }} \tilde{\mu}_{s s}^{l}>\frac{1}{1-\delta_{\max }} \tilde{\mu}_{b t}^{l} .
$$

From Proposition 4.8, we obtain $P\left(g_{b t}^{l}\right)<P\left(g_{s s}^{l}\right)$.

\section{Proof of Proposition 4.12;}

Rewrite $P\left(g^{l}\right)$ in Equation A.3 as

$$
\begin{aligned}
P\left(g^{l}\right)= & \operatorname{sum}\left(\frac{1}{K} E\right)+\operatorname{sum}\left(\frac{1}{K} \frac{\Gamma-\Theta}{\operatorname{det}(I-\Gamma)} E\right) \\
& +\operatorname{sum}\left(\frac{1}{K} \frac{\sum_{i=3}^{K} \Gamma^{i-1}}{\operatorname{det}(I-\Gamma)} E-\frac{1}{K} \frac{\sum_{i=2}^{K-1} \Gamma^{i-1}}{\operatorname{det}(I-\Gamma)} \Theta E\right) .
\end{aligned}
$$


Since elements of $\sum_{i=3}^{K} \Gamma^{i-1}, \sum_{i=2}^{K-1} \Gamma^{i-1} \Theta$ and $\operatorname{det}(I-\Gamma)$ are polynomials with $\delta_{i j}$ whose orders are no smaller than 2, we have

$$
P\left(g^{l}\right)=\operatorname{sum}\left(\frac{1}{K} E\right)+\operatorname{sum}\left(\frac{1}{K} \frac{\Gamma-\Theta}{\operatorname{det}(I-\Gamma)} E\right)+o\left(\delta_{\max }^{2}\right) .
$$

For batch policy and strictly sequencing policy, it can be rewritten as

$$
\begin{aligned}
& P\left(g_{b t}^{l}\right)=\frac{\sum_{i=1}^{n}\left(k_{s_{i}^{l}}-1\right) e_{s_{i}^{l}, s_{i}^{l}}+\sum_{i=2}^{n} e_{s_{i}^{l}, s_{i-1}^{l}}+e_{s_{1}^{l}, s_{n}^{l}}}{K} \\
& +\frac{\left(\delta_{s_{1}^{l}, s_{1}^{l}}-\delta_{s_{1}^{l}, s_{n}^{l}}\right) e_{s_{1}^{l}, s_{n}^{l}}+\sum_{i=2}^{n-1}\left(\delta_{s_{i}^{l}, s_{i}^{l}}-\delta_{s_{i}^{l}, s_{i-1}^{l}}\right) e_{s_{i}^{l}, s_{i-1}^{l}}}{K\left(1-\delta_{s_{1}^{l}, s_{n}^{l}} \prod_{i=2}^{n} \delta_{s_{i}^{l}, s_{i-1}^{l}} \prod_{i=1}^{n} \delta_{s_{i}^{l}, s_{i}^{l}}^{k_{l}^{l}-1}\right)} \\
& \left.+\frac{\left(\delta_{s_{n}^{l}, s_{n}^{l}}-\delta_{s_{n}^{l}, s_{n-1}^{l}}\right) e_{s_{n}^{l}, s_{n-1}^{l}}+\sum_{i=1}^{n-1}\left(\delta_{s_{i+1}^{l}, s_{i}^{l}}-\delta_{s_{i}^{l}, s_{i}^{l}}\right) e_{s_{i}^{l}, s_{i}^{l}}}{K\left(1-\delta_{s_{1}^{l}, s_{n}^{l}} \prod_{i=2}^{n} \delta_{s_{i}^{l}, s_{i-1}^{l}} \prod_{i=1}^{n} \delta_{s_{i}^{l},-1}^{l}, s_{i}^{l}\right.}\right) \\
& +\frac{\left(\delta_{s_{1}^{l}, s_{n}^{l}}-\delta_{s_{n}^{l}, s_{n}^{l}}\right) e_{s_{n}^{l}, s_{n}^{l}}}{K\left(1-\delta_{s_{1}^{l}, s_{n}^{l}} \prod_{i=2}^{n} \delta_{s_{i}^{l}, s_{i-1}^{l}} \prod_{i=1}^{n} \delta_{s_{i}^{l}, s_{i}^{l}}^{k_{s_{l}^{l}-1}}\right)} \\
& +o\left(\delta_{\max }^{2}\right) \text {, } \\
& P\left(g_{s s}^{l}\right)=\frac{\sum_{i=2}^{n} e_{s_{i}^{l}, s_{i-1}^{l}}+e_{s_{1}^{l}, s_{n}^{l}}}{n} \\
& +\frac{\left(\delta_{s_{2}^{l}, s_{1}^{l}}-\delta_{s_{1}^{l}, s_{n}^{l}}\right) e_{s_{1}^{l}, s_{n}^{l}}+\sum_{i=2}^{n-1}\left(\delta_{s_{i+1}^{l}, s_{i}^{l}}-\delta_{s_{i}^{l}, s_{i-1}^{l}}\right) e_{s_{i}^{l}, s_{i-1}^{l}}}{n\left(1-\delta_{s_{1}^{l}, s_{n}^{l}} \prod_{i=2}^{n} \delta_{s_{i}^{l}, s_{i-1}^{l}}\right)} \\
& +\frac{\left(\delta_{s_{1}^{l}, s_{n}^{l}}-\delta_{s_{n}^{l}, s_{n-1}^{l}}\right) e_{s_{n}^{l}, s_{n-1}^{l}}}{n\left(1-\delta_{s_{1}^{l}, s_{n}^{l}} \prod_{i=2}^{n} \delta_{s_{i}^{l}, s_{i-1}^{l}}\right)}+o\left(\delta_{\max }^{2}\right) \text {. }
\end{aligned}
$$

\section{Proof of Proposition 4.13:}

The good part probability is calculated by

$$
P\left(g^{l}\right)=\operatorname{sum}\left(\frac{1}{K} \frac{\sum_{i=1}^{K} \Gamma^{i-1}}{\operatorname{det}(I-\Gamma)} \Phi\right) .
$$


Define $\Theta$ and $E$ for batch policy

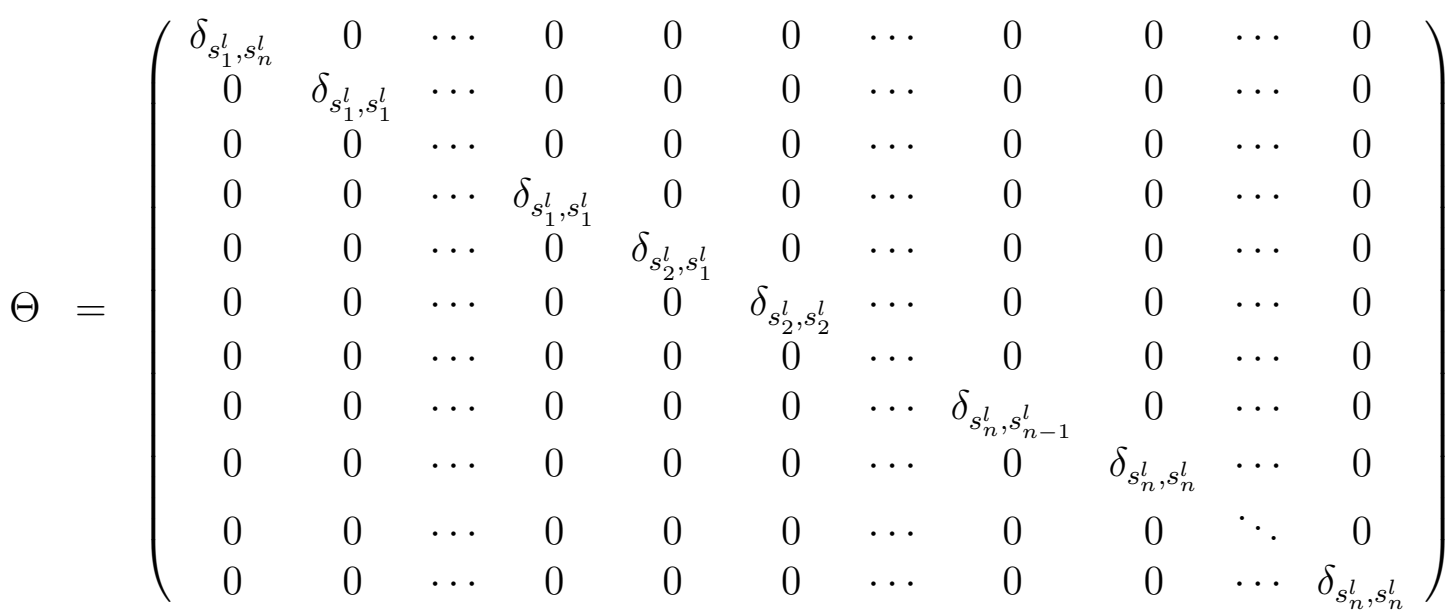

$$
\begin{aligned}
& E=\left[e_{s_{1}^{l}, s_{n}^{l}}, e_{s_{1}^{l}, s_{1}^{l}}, \ldots, e_{s_{1}^{l}, s_{1}^{l}}, e_{s_{2}^{l}, s_{1}^{l}}, e_{s_{2}^{l}, s_{2}^{l}}, \ldots, e_{s_{n}^{l}, s_{n-1}^{l}}, e_{s_{n}^{l}, s_{n}^{l}}, \ldots, e_{s_{n}^{l}, s_{n}^{l}}\right]^{T}
\end{aligned}
$$

and $\Theta$ and $E$ for strictly sequencing policy

$$
\begin{aligned}
\Theta & =\left(\begin{array}{cccc}
\delta_{s_{1}^{l}, s_{n}^{l}} & 0 & \cdots & 0 \\
0 & \delta_{s_{2}^{l}, s_{1}^{l}} & \cdots & 0 \\
0 & 0 & \ddots & 0 \\
0 & 0 & \cdots & \delta_{s_{n}^{l}, s_{n-1}^{l}}
\end{array}\right) \\
E & =\left[e_{s_{1}^{l}, s_{n}^{l}}, e_{s_{2}^{l}, s_{1}^{l}}, \ldots, e_{s_{n}^{l}, s_{n-1}^{l}}\right]^{T}
\end{aligned}
$$

Then, it follows that

$$
\Phi=(I-\Theta) E
$$

Furthermore,

$$
\begin{aligned}
P\left(g^{l}\right) & =\operatorname{sum}\left(\frac{1}{K} \frac{\sum_{i=1}^{K} \Gamma^{i-1}}{\operatorname{det}(I-\Gamma)}(I-\Theta) E\right) \\
& =\operatorname{sum}\left(\frac{1}{K} \frac{\sum_{i=1}^{K} \Gamma^{i-1}}{\operatorname{det}(I-\Gamma)} E-\frac{1}{K} \frac{\sum_{i=1}^{K} \Gamma^{i-1}}{\operatorname{det}(I-\Gamma)} \Theta E\right) \\
& =\operatorname{sum}\left(\frac{1}{K} \frac{I-\Gamma^{K-1} \Theta}{\operatorname{det}(I-\Gamma)} E+\frac{1}{K} \frac{\sum_{i=2}^{K} \Gamma^{i-1}}{\operatorname{det}(I-\Gamma)} E-\frac{1}{K} \frac{\sum_{i=1}^{K-1} \Gamma^{i-1}}{\operatorname{det}(I-\Gamma)} \Theta E\right)
\end{aligned}
$$

Since

$$
\operatorname{sum}\left(\left(I-\Gamma^{K-1} \Theta\right) E\right)=\operatorname{det}(I-\Gamma) \operatorname{sum}(E),
$$


we can obtain

$$
P\left(g^{l}\right)=\operatorname{sum}\left(\frac{1}{K} E\right)+\operatorname{sum}\left(\frac{1}{K} \frac{\sum_{i=2}^{K} \Gamma^{i-1}}{\operatorname{det}(I-\Gamma)} E-\frac{1}{K} \frac{\sum_{i=1}^{K-1} \Gamma^{i-1}}{\operatorname{det}(I-\Gamma)} \Theta E\right) .
$$

It can be shown that $\sum_{i=2}^{K} \Gamma^{i-1}, \sum_{i=1}^{K-1} \Gamma^{i-1} \Theta$ and $\operatorname{det}(I-\Gamma)$ are polynomials with $\delta_{i j}$ whose orders are no smaller than 1 . Thus,

$$
P\left(g^{l}\right)=\operatorname{sum}\left(\frac{1}{K} E\right)+o\left(\delta_{\max }\right)
$$

\section{Proof of Corollary 4.2:}

From Equation (A.1), we obtain

$$
\left|\operatorname{sum}\left(\frac{1}{K} \frac{\Gamma^{i}-\Gamma^{i-1} \Theta}{\operatorname{det}(I-\Gamma)} E\right)\right| \leq \frac{2 \delta_{\max }^{i}}{1-\delta_{\max }^{K}} \operatorname{sum}\left(\frac{1}{K} E\right)
$$

Then,

$$
\begin{aligned}
& \left|o\left(\delta_{\max }^{2}\right)\right|=\left|\operatorname{sum}\left(\frac{1}{K} \frac{\sum_{i=3}^{K} \Gamma^{i-1}}{\operatorname{det}(I-\Gamma)} E-\frac{1}{K} \frac{\sum_{i=2}^{K-1} \Gamma^{i-1}}{\operatorname{det}(I-\Gamma)} \Theta E\right)\right| \\
& =\left|\operatorname{sum}\left(\frac{1}{K} \frac{\sum_{i=2}^{K-1}\left(\Gamma^{i}-\Gamma^{i-1} \Theta\right)}{\operatorname{det}(I-\Gamma)} E\right)\right| \\
& =\left|\sum_{i=2}^{K-1} \operatorname{sum}\left(\frac{1}{K} \frac{\Gamma^{i}-\Gamma^{i-1} \Theta}{\operatorname{det}(I-\Gamma)} E\right)\right| \\
& \leq \sum_{i=2}^{K-1}\left|\operatorname{sum}\left(\frac{1}{K} \frac{\Gamma^{i}-\Gamma^{i-1} \Theta}{\operatorname{det}(I-\Gamma)} E\right)\right| \\
& \leq \sum_{i=2}^{K-1} \frac{2 \delta_{\max }^{i}}{1-\delta_{\max }^{K}} \operatorname{sum}\left(\frac{1}{K} E\right) \\
& =2\left(\frac{1}{1-\delta_{\max }}-\frac{1+\delta_{\max }}{1-\delta_{\max }^{K}}\right) \operatorname{sum}\left(\frac{1}{K} E\right) \text {. }
\end{aligned}
$$




\section{Proof of Corollary 4.3 :}

$$
\begin{aligned}
\left|o\left(\delta_{\max }\right)\right| & =\left|\operatorname{sum}\left(\frac{1}{K} \frac{\sum_{i=2}^{K} \Gamma^{i-1}}{\operatorname{det}(I-\Gamma)} E-\frac{1}{K} \frac{\sum_{i=1}^{K-1} \Gamma^{i-1}}{\operatorname{det}(I-\Gamma)} \Theta E\right)\right| \\
& =\left|\operatorname{sum}\left(\frac{1}{K} \frac{\sum_{i=1}^{K-1}\left(\Gamma^{i}-\Gamma^{i-1} \Theta\right)}{\operatorname{det}(I-\Gamma)} E\right)\right| \\
& =\left|\sum_{i=1}^{K-1} \operatorname{sum}\left(\frac{1}{K} \frac{\Gamma^{i}-\Gamma^{i-1} \Theta}{\operatorname{det}(I-\Gamma)} E\right)\right| \\
& \leq \sum_{i=1}^{K-1}\left|\operatorname{sum}\left(\frac{1}{K} \frac{\Gamma^{i}-\Gamma^{i-1} \Theta}{\operatorname{det}(I-\Gamma)} E\right)\right| \\
& \leq \sum_{i=1}^{K-1} \frac{2 \delta_{\max }^{i}}{1-\delta_{\max }^{K}} \operatorname{sum}\left(\frac{1}{K} E\right) \\
& =2\left(\frac{1}{1-\delta_{\max }}-\frac{1}{1-\delta_{\max }^{K}}\right) \operatorname{sum}\left(\frac{1}{K} E\right) .
\end{aligned}
$$

\section{Appendix C: Proofs of Chapter 5}

\section{Proof of Proposition 5.1:}

Assume the product sequence is 1-2-3-1. Then the transition equations can be written as follows:

$$
\begin{aligned}
& P\left(g_{11}\right)=\delta_{13} P\left(g_{31}\right)+\frac{1}{3} \mu_{13}, \\
& P\left(g_{21}\right)=\delta_{21} P\left(g_{11}\right)+\frac{1}{3} \mu_{21}, \\
& P\left(g_{31}\right)=\delta_{32} P\left(g_{21}\right)+\frac{1}{3} \mu_{32} .
\end{aligned}
$$

Consider a subtle increment $\Delta \lambda_{21}$ of $\lambda_{21}$. Then, we have

$$
\begin{aligned}
& P\left(g_{11}\right)+\Delta P\left(g_{11}\right)=\delta_{13}\left[P\left(g_{31}\right)+\Delta P\left(g_{31}\right)\right]+\frac{1}{3} \mu_{13}, \\
& P\left(g_{21}\right)+\Delta P\left(g_{21}\right)=\left(\delta_{21}-\Delta \lambda_{21}\right)\left[P\left(g_{11}\right)+\Delta P\left(g_{11}\right)\right]+\frac{1}{3} \mu_{21}, \\
& P\left(g_{31}\right)+\Delta P\left(g_{31}\right)=\delta_{32}\left[P\left(g_{21}\right)+\Delta P\left(g_{11}\right)\right]+\frac{1}{3} \mu_{32} .
\end{aligned}
$$


By subtracting the transition equations after subtle change with the ones before change, we obtain

$$
\begin{aligned}
& \Delta P\left(g_{11}\right)=\delta_{13} \Delta P\left(g_{31}\right), \\
& \Delta P\left(g_{21}\right)=-\Delta \lambda_{21} P\left(g_{11}\right)+\left(\delta_{21}-\Delta \lambda_{21}\right) \Delta P\left(g_{11}\right), \\
& \Delta P\left(g_{31}\right)=\delta_{32} \Delta P\left(g_{21}\right) .
\end{aligned}
$$

Since

$$
\lim _{\Delta \lambda_{21} \longrightarrow 0} \frac{\Delta P\left(g_{i j}\right)}{\Delta \lambda_{21}}=\frac{\partial P\left(g_{i j}\right)}{\partial \lambda_{21}},
$$

we have

$$
\begin{aligned}
& \frac{\partial P\left(g_{11}\right)}{\partial \lambda_{21}}=\delta_{13} \frac{\partial P\left(g_{31}\right)}{\partial \lambda_{21}} \\
& \frac{\partial P\left(g_{21}\right)}{\partial \lambda_{21}}=-P\left(g_{11}\right)+\delta_{21} \frac{\partial P\left(g_{11}\right)}{\partial \lambda_{21}} \\
& \frac{\partial P\left(g_{31}\right)}{\partial \lambda_{21}}=\delta_{32} \frac{\partial P\left(g_{21}\right)}{\partial \lambda_{21}} .
\end{aligned}
$$

Thus,

$$
\frac{\partial P\left(g_{21}\right)}{\partial \lambda_{21}}=-\frac{P\left(g_{11}\right)}{1-\delta_{21} \delta_{13} \delta_{32}} .
$$

The partial derivative of $P\left(g_{s s}\right)$ with respect to $\lambda_{21}$ is

$$
\begin{aligned}
\frac{\partial P\left(g_{s s}\right)}{\partial \lambda_{21}} & =\frac{\partial P\left(g_{21}\right)}{\partial \lambda_{21}}+\frac{\partial P\left(g_{31}\right)}{\partial \lambda_{21}}+\frac{\partial P\left(g_{11}\right)}{\partial \lambda_{31}} \\
& =\frac{\partial P\left(g_{21}\right)}{\partial \lambda_{21}}\left(1+\delta_{32}+\delta_{32} \delta_{13}\right) \\
& =-\frac{P\left(g_{11}\right)}{1-\delta_{21} \delta_{13} \delta_{32}}\left(1+\delta_{32}+\delta_{32} \delta_{13}\right) .
\end{aligned}
$$

Similarly, consider a subtle increment $\Delta \mu_{21}$ of $\mu_{21}$. Then, we have

$$
P\left(g_{11}\right)+\Delta P\left(g_{11}\right)=\delta_{13}\left[P\left(g_{31}\right)+\Delta P\left(g_{31}\right)\right]+\frac{1}{3} \mu_{13},
$$




$$
\begin{aligned}
& P\left(g_{21}\right)+\Delta P\left(g_{21}\right)=\left(\delta_{21}-\Delta \mu_{21}\right)\left[P\left(g_{11}\right)+\Delta P\left(g_{11}\right)\right]+\frac{1}{3}\left(\mu_{21}+\Delta \mu_{21}\right), \\
& P\left(g_{31}\right)+\Delta P\left(g_{31}\right)=\delta_{32}\left[P\left(g_{21}\right)+\Delta P\left(g_{11}\right)\right]+\frac{1}{3} \mu_{32} .
\end{aligned}
$$

By subtracting the transition equations after subtle change with the ones before change, we obtain

$$
\begin{aligned}
& \Delta P\left(g_{11}\right)=\delta_{13} \Delta P\left(g_{31}\right), \\
& \Delta P\left(g_{21}\right)=-\Delta \mu_{21}\left[P\left(g_{11}\right)-\frac{1}{3}\right]+\left(\delta_{21}-\Delta \mu_{21}\right) \Delta P\left(g_{11}\right), \\
& \Delta P\left(g_{31}\right)=\delta_{32} \Delta P\left(g_{21}\right) .
\end{aligned}
$$

Thus,

$$
\frac{\partial P\left(g_{21}\right)}{\partial \mu_{21}}=-\frac{P\left(g_{11}\right)-\frac{1}{3}}{1-\delta_{21} \delta_{13} \delta_{32}} .
$$

The partial derivative of $P\left(g_{s s}\right)$ with respect to $\mu_{21}$ is

$$
\begin{aligned}
\frac{\partial P\left(g_{s s}\right)}{\partial \mu_{21}} & =\frac{\partial P\left(g_{21}\right)}{\partial \mu_{21}}+\frac{\partial P\left(g_{31}\right)}{\partial \mu_{21}}+\frac{\partial P\left(g_{11}\right)}{\partial \mu_{31}} \\
& =\frac{\partial P\left(g_{21}\right)}{\partial \mu_{21}}\left(1+\delta_{32}+\delta_{32} \delta_{13}\right) \\
& =-\frac{P\left(g_{11}\right)-\frac{1}{3}}{1-\delta_{21} \delta_{13} \delta_{32}}\left(1+\delta_{32}+\delta_{32} \delta_{13}\right) .
\end{aligned}
$$

Since $P\left(g_{11}\right)+P\left(d_{11}\right)=\frac{1}{3}$, the equation can be rewritten as

$$
\frac{\partial P\left(g_{s s}\right)}{\partial \mu_{21}}=\frac{P\left(d_{11}\right)}{1-\delta_{21} \delta_{13} \delta_{32}}\left(1+\delta_{32}+\delta_{32} \delta_{13}\right)
$$

Following similar derivation, the conclusions hold.

\section{Proof of Proposition 5.2:}

Assume there are two types of products, each with batch size three. The product sequence will be 1-1-1-2-2-2-1. Similar with strict sequence, the transition equations 
with good states only can be written as follows:

$$
\begin{aligned}
& P\left(g_{11}\right)=\delta_{12} P\left(g_{23}\right)+\frac{1}{6} \mu_{12}, \\
& P\left(g_{12}\right)=\delta_{11} P\left(g_{11}\right)+\frac{1}{6} \mu_{11}, \\
& P\left(g_{13}\right)=\delta_{11} P\left(g_{12}\right)+\frac{1}{6} \mu_{11}, \\
& P\left(g_{21}\right)=\delta_{21} P\left(g_{13}\right)+\frac{1}{6} \mu_{21}, \\
& P\left(g_{22}\right)=\delta_{22} P\left(g_{21}\right)+\frac{1}{6} \mu_{22}, \\
& P\left(g_{23}\right)=\delta_{22} P\left(g_{22}\right)+\frac{1}{6} \mu_{22} .
\end{aligned}
$$

In batch policy, transitions with product switch appear once while transitions without product switch happen more as batch sizes increase. We investigate transitions with switch first and then without switch.

\section{Transitions with switch}

Consider a subtle increment $\Delta \lambda_{21}$ of $\lambda_{21}$. Then, we have

$$
\begin{aligned}
& P\left(g_{11}\right)+\Delta P\left(g_{11}\right)=\delta_{12}\left[P\left(g_{23}\right)+\Delta P\left(g_{23}\right)\right]+\frac{1}{6} \mu_{12}, \\
& P\left(g_{12}\right)+\Delta P\left(g_{12}\right)=\delta_{11}\left[P\left(g_{11}\right)+\Delta P\left(g_{11}\right)\right]+\frac{1}{6} \mu_{11}, \\
& P\left(g_{13}\right)+\Delta P\left(g_{13}\right)=\delta_{11}\left[P\left(g_{12}\right)+\Delta P\left(g_{12}\right)\right]+\frac{1}{6} \mu_{11}, \\
& P\left(g_{21}\right)+\Delta P\left(g_{21}\right)=\left(\delta_{21}-\Delta \lambda_{21}\right)\left[P\left(g_{13}\right)+\Delta P\left(g_{13}\right)\right]+\frac{1}{6} \mu_{21}, \\
& P\left(g_{22}\right)+\Delta P\left(g_{22}\right)=\delta_{22}\left[P\left(g_{21}\right)+\Delta P\left(g_{21}\right)\right]+\frac{1}{6} \mu_{22}, \\
& P\left(g_{23}\right)+\Delta P\left(g_{23}\right)=\delta_{22}\left[P\left(g_{22}\right)+\Delta P\left(g_{22}\right)\right]+\frac{1}{6} \mu_{22} .
\end{aligned}
$$


By subtracting the transition equations after subtle change with the ones before change, we obtain

$$
\begin{aligned}
& \Delta P\left(g_{11}\right)=\delta_{12} \Delta P\left(g_{23}\right), \\
& \Delta P\left(g_{12}\right)=\delta_{11} \Delta P\left(g_{11}\right), \\
& \Delta P\left(g_{13}\right)=\delta_{11} \Delta P\left(g_{12}\right), \\
& \Delta P\left(g_{21}\right)=-\Delta \lambda_{21} P\left(g_{13}\right)+\left(\delta_{21}-\Delta \lambda_{21}\right) \Delta P\left(g_{13}\right), \\
& \Delta P\left(g_{22}\right)=\delta_{22} \Delta P\left(g_{21}\right), \\
& \Delta P\left(g_{23}\right)=\delta_{22} \Delta P\left(g_{22}\right) .
\end{aligned}
$$

Since

$$
\lim _{\Delta \lambda_{21} \longrightarrow 0} \frac{\Delta P\left(g_{i j}\right)}{\Delta \lambda_{21}}=\frac{\partial P\left(g_{i j}\right)}{\partial \lambda_{21}},
$$

we have

$$
\begin{aligned}
& \frac{\partial P\left(g_{11}\right)}{\partial \lambda_{21}}=\delta_{12} \frac{\partial P\left(g_{23}\right)}{\partial \lambda_{21}} \\
& \frac{\partial P\left(g_{12}\right)}{\partial \lambda_{21}}=\delta_{11} \frac{\partial P\left(g_{11}\right)}{\partial \lambda_{21}} \\
& \frac{\partial P\left(g_{13}\right)}{\partial \lambda_{21}}=\delta_{11} \frac{\partial P\left(g_{12}\right)}{\partial \lambda_{21}} \\
& \frac{\partial P\left(g_{21}\right)}{\partial \lambda_{21}}=-P\left(g_{13}\right)+\delta_{21} \frac{\partial P\left(g_{13}\right)}{\partial \lambda_{21}} \\
& \frac{\partial P\left(g_{22}\right)}{\partial \lambda_{21}}=\delta_{22} \frac{\partial P\left(g_{21}\right)}{\partial \lambda_{21}} \\
& \frac{\partial P\left(g_{23}\right)}{\partial \lambda_{21}}=\delta_{22} \frac{\partial P\left(g_{22}\right)}{\partial \lambda_{21}} .
\end{aligned}
$$

Thus,

$$
\frac{\partial P\left(g_{21}\right)}{\partial \lambda_{21}}=-\frac{P\left(g_{13}\right)}{1-\delta_{21} \delta_{11}^{2} \delta_{12} \delta_{22}^{2}} .
$$


The partial derivative of $P\left(g_{b t}\right)$ with respect to $\lambda_{21}$ is

$$
\begin{aligned}
\frac{\partial P\left(g_{b t}\right)}{\partial \lambda_{21}} & =\frac{\partial P\left(g_{21}\right)}{\partial \lambda_{21}}+\frac{\partial P\left(g_{22}\right)}{\partial \lambda_{21}}+\frac{\partial P\left(g_{23}\right)}{\partial \lambda_{31}}+\frac{\partial P\left(g_{21}\right)}{\partial \lambda_{21}}+\frac{\partial P\left(g_{22}\right)}{\partial \lambda_{11}}+\frac{\partial P\left(g_{12}\right)}{\partial \lambda_{13}} \\
& =\frac{\partial P\left(g_{21}\right)}{\partial \lambda_{21}}\left(1+\delta_{22}+\delta_{22}^{2}+\delta_{22}^{2} \delta_{12}+\delta_{22}^{2} \delta_{12} \delta_{11}+\delta_{22}^{2} \delta_{12} \delta_{11}^{2}\right) \\
& =-\frac{P\left(g_{13}\right)}{1-\delta_{21} \delta_{11}^{2} \delta_{12} \delta_{22}^{2}}\left(1+\delta_{22}+\delta_{22}^{2}+\delta_{22}^{2} \delta_{12}+\delta_{22}^{2} \delta_{12} \delta_{11}+\delta_{22}^{2} \delta_{12} \delta_{11}^{2}\right) .
\end{aligned}
$$

Similarly, the partial derivative of $P\left(g_{b t}\right)$ with respect to $\mu_{21}$ is

$$
\begin{aligned}
\frac{\partial P\left(g_{b t}\right)}{\partial \mu_{21}} & =\frac{\partial P\left(g_{21}\right)}{\partial \mu_{21}}+\frac{\partial P\left(g_{22}\right)}{\partial \mu_{21}}+\frac{\partial P\left(g_{23}\right)}{\partial \mu_{31}}+\frac{\partial P\left(g_{21}\right)}{\partial \mu_{21}}+\frac{\partial P\left(g_{22}\right)}{\partial \mu_{11}}+\frac{\partial P\left(g_{12}\right)}{\partial \mu_{13}} \\
& =\frac{\partial P\left(g_{21}\right)}{\partial \mu_{21}}\left(1+\delta_{22}+\delta_{22}^{2}+\delta_{22}^{2} \delta_{12}+\delta_{22}^{2} \delta_{12} \delta_{11}+\delta_{22}^{2} \delta_{12} \delta_{11}^{2}\right) \\
& =\frac{P\left(d_{13}\right)}{1-\delta_{21} \delta_{11}^{2} \delta_{12} \delta_{22}^{2}}\left(1+\delta_{22}+\delta_{22}^{2}+\delta_{22}^{2} \delta_{12}+\delta_{22}^{2} \delta_{12} \delta_{11}+\delta_{22}^{2} \delta_{12} \delta_{11}^{2}\right) .
\end{aligned}
$$

\section{Transitions without switch}

Consider a subtle increment $\Delta \lambda_{11}$ of $\lambda_{11}$. Then, we have

$$
\begin{aligned}
& P\left(g_{11}\right)+\Delta P\left(g_{11}\right)=\delta_{12}\left[P\left(g_{23}\right)+\Delta P\left(g_{23}\right)\right]+\frac{1}{6} \mu_{12}, \\
& P\left(g_{12}\right)+\Delta P\left(g_{12}\right)=\left(\delta_{11}-\Delta \lambda_{11}\right)\left[P\left(g_{11}\right)+\Delta P\left(g_{11}\right)\right]+\frac{1}{6} \mu_{11}, \\
& P\left(g_{13}\right)+\Delta P\left(g_{13}\right)=\left(\delta_{11}-\Delta \lambda_{11}\right)\left[P\left(g_{12}\right)+\Delta P\left(g_{12}\right)\right]+\frac{1}{6} \mu_{11}, \\
& P\left(g_{21}\right)+\Delta P\left(g_{21}\right)=\delta_{21}\left[P\left(g_{13}\right)+\Delta P\left(g_{13}\right)\right]+\frac{1}{6} \mu_{21}, \\
& P\left(g_{22}\right)+\Delta P\left(g_{22}\right)=\delta_{22}\left[P\left(g_{21}\right)+\Delta P\left(g_{21}\right)\right]+\frac{1}{6} \mu_{22}, \\
& P\left(g_{23}\right)+\Delta P\left(g_{23}\right)=\delta_{22}\left[P\left(g_{22}\right)+\Delta P\left(g_{22}\right)\right]+\frac{1}{6} \mu_{22} .
\end{aligned}
$$

By subtracting the transition equations after subtle change with the ones before change, we obtain

$$
\Delta P\left(g_{11}\right)=\delta_{12} \Delta P\left(g_{23}\right)
$$




$$
\begin{aligned}
& \Delta P\left(g_{12}\right)=-\Delta \lambda_{11} P\left(g_{11}\right)+\left(\delta_{11}-\Delta \lambda_{11}\right) \Delta P\left(g_{11}\right), \\
& \Delta P\left(g_{13}\right)=-\Delta \lambda_{11} P\left(g_{12}\right)+\left(\delta_{11}-\Delta \lambda_{11}\right) \Delta P\left(g_{12}\right), \\
& \Delta P\left(g_{21}\right)=\delta_{21} \Delta P\left(g_{13}\right), \\
& \Delta P\left(g_{22}\right)=\delta_{22} \Delta P\left(g_{21}\right), \\
& \Delta P\left(g_{23}\right)=\delta_{22} \Delta P\left(g_{22}\right) .
\end{aligned}
$$

Since

$$
\lim _{\Delta \lambda_{21} \longrightarrow 0} \frac{\Delta P\left(g_{i j}\right)}{\Delta \lambda_{21}}=\frac{\partial P\left(g_{i j}\right)}{\partial \lambda_{21}},
$$

we have

$$
\begin{aligned}
& \frac{\partial P\left(g_{11}\right)}{\partial \lambda_{11}}=\delta_{12} \frac{\partial P\left(g_{23}\right)}{\partial \lambda_{11}} \\
& \frac{\partial P\left(g_{12}\right)}{\partial \lambda_{11}}=-P\left(g_{11}\right)+\delta_{11} \frac{\partial P\left(g_{11}\right)}{\partial \lambda_{11}} \\
& \frac{\partial P\left(g_{13}\right)}{\partial \lambda_{11}}=-P\left(g_{12}\right)+\delta_{11} \frac{\partial P\left(g_{12}\right)}{\partial \lambda_{11}} \\
& \frac{\partial P\left(g_{21}\right)}{\partial \lambda_{11}}=\delta_{21} \frac{\partial P\left(g_{13}\right)}{\partial \lambda_{11}} \\
& \frac{\partial P\left(g_{22}\right)}{\partial \lambda_{11}}=\delta_{22} \frac{\partial P\left(g_{21}\right)}{\partial \lambda_{11}} \\
& \frac{\partial P\left(g_{23}\right)}{\partial \lambda_{11}}=\delta_{22} \frac{\partial P\left(g_{22}\right)}{\partial \lambda_{11}} .
\end{aligned}
$$

Thus,

$$
\frac{\partial P\left(g_{13}\right)}{\partial \lambda_{11}}=-\frac{P\left(g_{12}\right)+\delta_{11} P\left(g_{11}\right)}{1-\delta_{21} \delta_{11}^{2} \delta_{12} \delta_{22}^{2}} .
$$

The partial derivative of $P\left(g_{b t}\right)$ with respect to $\lambda_{11}$ is

$$
\begin{aligned}
\frac{\partial P\left(g_{b t}\right)}{\partial \lambda_{11}} & =\frac{\partial P\left(g_{13}\right)}{\partial \lambda_{11}}+\frac{\partial P\left(g_{21}\right)}{\partial \lambda_{11}}+\frac{\partial P\left(g_{22}\right)}{\partial \lambda_{11}}+\frac{\partial P\left(g_{23}\right)}{\partial \lambda_{11}}+\frac{\partial P\left(g_{11}\right)}{\partial \lambda_{11}}+\frac{\partial P\left(g_{12}\right)}{\partial \lambda_{11}} \\
& =\frac{\partial P\left(g_{13}\right)}{\partial \lambda_{11}}\left(1+\delta_{21}+\delta_{21} \delta_{22}+\delta_{21} \delta_{22}^{2}+\delta_{21} \delta_{22}^{2} \delta_{12}+\delta_{21} \delta_{22}^{2} \delta_{12} \delta_{11}\right)-P\left(g_{11}\right)
\end{aligned}
$$




$$
\begin{aligned}
= & -\frac{P\left(g_{12}\right)}{1-\delta_{21} \delta_{11}^{2} \delta_{12} \delta_{22}^{2}}\left(1+\delta_{21}+\delta_{21} \delta_{22}+\delta_{21} \delta_{22}^{2}+\delta_{21} \delta_{22}^{2} \delta_{12}+\delta_{21} \delta_{22}^{2} \delta_{12} \delta_{11}\right) \\
& -\frac{P\left(g_{11}\right)}{1-\delta_{21} \delta_{11}^{2} \delta_{12} \delta_{22}^{2}}\left(1+\delta_{11}+\delta_{11} \delta_{21}+\delta_{11} \delta_{21} \delta_{22}+\delta_{11} \delta_{21} \delta_{22}^{2}+\delta_{11} \delta_{21} \delta_{22}^{2} \delta_{12}\right) .
\end{aligned}
$$

Similarly, consider a subtle increment $\Delta \mu_{11}$ of $\mu_{11}$. Then, we have

$$
\begin{aligned}
& P\left(g_{11}\right)+\Delta P\left(g_{11}\right)=\delta_{12}\left[P\left(g_{23}\right)+\Delta P\left(g_{23}\right)\right]+\frac{1}{6} \mu_{12}, \\
& P\left(g_{12}\right)+\Delta P\left(g_{12}\right)=\left(\delta_{11}-\Delta \mu_{11}\right)\left[P\left(g_{11}\right)+\Delta P\left(g_{11}\right)\right]+\frac{1}{6}\left(\mu_{11}+\Delta \mu_{11}\right), \\
& P\left(g_{13}\right)+\Delta P\left(g_{13}\right)=\left(\delta_{11}-\Delta \mu_{11}\right)\left[P\left(g_{12}\right)+\Delta P\left(g_{12}\right)\right]+\frac{1}{6}\left(\mu_{11}+\Delta \mu_{11}\right), \\
& P\left(g_{21}\right)+\Delta P\left(g_{21}\right)=\delta_{21}\left[P\left(g_{13}\right)+\Delta P\left(g_{13}\right)\right]+\frac{1}{6} \mu_{21}, \\
& P\left(g_{22}\right)+\Delta P\left(g_{22}\right)=\delta_{22}\left[P\left(g_{21}\right)+\Delta P\left(g_{21}\right)\right]+\frac{1}{6} \mu_{22}, \\
& P\left(g_{23}\right)+\Delta P\left(g_{23}\right)=\delta_{22}\left[P\left(g_{22}\right)+\Delta P\left(g_{22}\right)\right]+\frac{1}{6} \mu_{22} .
\end{aligned}
$$

By subtracting the transition equations after subtle change with the ones before change, we obtain

$$
\begin{aligned}
& \Delta P\left(g_{11}\right)=\delta_{12} \Delta P\left(g_{23}\right), \\
& \Delta P\left(g_{12}\right)=-\Delta \mu_{11}\left[P\left(g_{11}\right)-\frac{1}{6}\right]+\left(\delta_{11}-\Delta \mu_{11}\right) \Delta P\left(g_{11}\right), \\
& \Delta P\left(g_{13}\right)=-\Delta \mu_{11}\left[P\left(g_{12}\right)-\frac{1}{6}\right]+\left(\delta_{11}-\Delta \mu_{11}\right) \Delta P\left(g_{12}\right), \\
& \Delta P\left(g_{21}\right)=\delta_{21} \Delta P\left(g_{13}\right), \\
& \Delta P\left(g_{22}\right)=\delta_{22} \Delta P\left(g_{21}\right), \\
& \Delta P\left(g_{23}\right)=\delta_{22} \Delta P\left(g_{22}\right) .
\end{aligned}
$$

When $\Delta \mu_{11} \longrightarrow 0$ and $P\left(g_{11}\right)+P\left(d_{11}\right)=P\left(g_{12}\right)+P\left(d_{12}\right)=\frac{1}{6}$, we have

$$
\frac{\partial P\left(g_{11}\right)}{\partial \mu_{11}}=\delta_{12} \frac{\partial P\left(g_{23}\right)}{\partial \mu_{11}},
$$




$$
\begin{aligned}
& \frac{\partial P\left(g_{12}\right)}{\partial \mu_{11}}=P\left(d_{11}\right)+\delta_{11} \frac{\partial P\left(g_{11}\right)}{\partial \mu_{11}}, \\
& \frac{\partial P\left(g_{13}\right)}{\partial \mu_{11}}=P\left(d_{12}\right)+\delta_{11} \frac{\partial P\left(g_{12}\right)}{\partial \mu_{11}}, \\
& \frac{\partial P\left(g_{21}\right)}{\partial \mu_{11}}=\delta_{21} \frac{\partial P\left(g_{13}\right)}{\partial \mu_{11}} \\
& \frac{\partial P\left(g_{22}\right)}{\partial \mu_{11}}=\delta_{22} \frac{\partial P\left(g_{21}\right)}{\partial \mu_{11}} \\
& \frac{\partial P\left(g_{23}\right)}{\partial \mu_{11}}=\delta_{22} \frac{\partial P\left(g_{22}\right)}{\partial \mu_{11}} .
\end{aligned}
$$

Thus,

$$
\frac{\partial P\left(g_{13}\right)}{\partial \mu_{11}}=\frac{P\left(d_{12}\right)+\delta_{11} P\left(d_{11}\right)}{1-\delta_{21} \delta_{11}^{2} \delta_{12} \delta_{22}^{2}} .
$$

The partial derivative of $P\left(g_{b t}\right)$ with respect to $\mu_{11}$ is

$$
\begin{aligned}
\frac{\partial P\left(g_{b t}\right)}{\partial \mu_{11}}= & \frac{\partial P\left(g_{13}\right)}{\partial \mu_{11}}+\frac{\partial P\left(g_{21}\right)}{\partial \mu_{11}}+\frac{\partial P\left(g_{22}\right)}{\partial \mu_{11}}+\frac{\partial P\left(g_{23}\right)}{\partial \mu_{11}}+\frac{\partial P\left(g_{11}\right)}{\partial \mu_{11}}+\frac{\partial P\left(g_{12}\right)}{\partial \mu_{11}} \\
= & \frac{\partial P\left(g_{13}\right)}{\partial \mu_{11}}\left(1+\delta_{21}+\delta_{21} \delta_{22}+\delta_{21} \delta_{22}^{2}+\delta_{21} \delta_{22}^{2} \delta_{12}+\delta_{21} \delta_{22}^{2} \delta_{12} \delta_{11}\right)+P\left(d_{11}\right) \\
= & \frac{P\left(d_{12}\right)}{1-\delta_{21} \delta_{11}^{2} \delta_{12} \delta_{22}^{2}}\left(1+\delta_{21}+\delta_{21} \delta_{22}+\delta_{21} \delta_{22}^{2}+\delta_{21} \delta_{22}^{2} \delta_{12}+\delta_{21} \delta_{22}^{2} \delta_{12} \delta_{11}\right) \\
& +\frac{P\left(d_{11}\right)}{1-\delta_{21} \delta_{11}^{2} \delta_{12} \delta_{22}^{2}}\left(1+\delta_{11}+\delta_{11} \delta_{21}+\delta_{11} \delta_{21} \delta_{22}+\delta_{11} \delta_{21} \delta_{22}^{2}+\delta_{11} \delta_{21} \delta_{22}^{2} \delta_{12}\right) .
\end{aligned}
$$

Following similar derivation, the conclusions hold.

\section{Proof of Proposition 5.3 and 5.4;}

$$
\begin{aligned}
P\left(g^{l}\right) & =\operatorname{sum}\left(\frac{1}{K}(I-\Gamma)^{-1} \Phi\right) \\
& =\operatorname{sum}\left(\frac{1}{K} \frac{\sum_{i=1}^{K} \Gamma^{i-1}}{\operatorname{det}(I-\Gamma)} \Phi\right) .
\end{aligned}
$$


where $\Gamma$ and $\Phi$ for batch policy

$$
\begin{aligned}
& \Gamma=\left(\begin{array}{ccccccccccc}
0 & \cdots & 0 & 0 & 0 & \cdots & 0 & 0 & \cdots & 0 & \delta_{s_{1}^{l}, s_{n}^{l}} \\
\delta_{s_{1}^{l}, s_{1}^{l}} & \cdots & 0 & 0 & 0 & \cdots & 0 & 0 & \cdots & 0 & 0 \\
0 & \ddots & 0 & 0 & 0 & \cdots & 0 & 0 & \cdots & 0 & 0 \\
0 & \cdots & \delta_{s_{1}^{l}, s_{1}^{l}} & 0 & 0 & \cdots & 0 & 0 & \cdots & 0 & 0 \\
0 & \cdots & 0 & \delta_{s_{2}^{l}, s_{1}^{l}} & 0 & \cdots & 0 & 0 & \cdots & 0 & 0 \\
0 & \cdots & 0 & 0 & \delta_{s_{2}, s_{2}^{l}} & \cdots & 0 & 0 & \cdots & 0 & 0 \\
0 & \cdots & 0 & 0 & 0 & \ddots & 0 & 0 & \cdots & 0 & 0 \\
0 & \cdots & 0 & 0 & 0 & \cdots & \delta_{s_{n}^{l}, s_{n-1}^{l}} & 0 & \cdots & 0 & 0 \\
0 & \cdots & 0 & 0 & 0 & \cdots & 0 & \delta_{s_{n}^{l}, s_{n}^{l}} & \cdots & 0 & 0 \\
0 & \cdots & 0 & 0 & 0 & \cdots & 0 & 0 & \ddots & 0 & 0 \\
0 & \cdots & 0 & 0 & 0 & \cdots & 0 & 0 & \cdots & \delta_{s_{n}^{l}, s_{n}^{l}} & 0
\end{array}\right), \\
& \Phi=\left[\mu_{s_{1}^{l}, s_{n}^{l}}, \mu_{s_{1}^{l}, s_{1}^{l}}, \ldots, \mu_{s_{1}^{l}, s_{1}^{l}}, \mu_{s_{2}^{l}, s_{1}^{l}}, \mu_{s_{2}^{l}, s_{2}^{l}}, \ldots, \mu_{s_{n}^{l}, s_{n-1}^{l}}, \mu_{s_{n}^{l}, s_{n}^{l}}, \ldots, \mu_{s_{n}^{l}, s_{n}^{l}}\right]^{T} \text {, }
\end{aligned}
$$

and $\Gamma$ and $\Phi$ for strictly sequencing policy

$$
\begin{aligned}
\Gamma & =\left(\begin{array}{cccc}
0 & \cdots & 0 & \delta_{s_{1}^{l}, s_{n}^{l}} \\
\delta_{s_{2}^{l}, s_{1}^{l}} & \cdots & 0 & 0 \\
0 & \ddots & 0 & 0 \\
0 & \cdots & \delta_{s_{n}^{l}, s_{n-1}^{l}} & 0
\end{array}\right), \\
\Phi & =\left[\mu_{s_{1}^{l}, s_{n}^{l}}, \mu_{s_{2}^{l}, s_{1}^{l}}, \ldots, \mu_{s_{n}^{l}, s_{n-1}^{l}}\right]^{T} .
\end{aligned}
$$

Step 1: Transform $P\left(g^{l}\right)$ as a equation of $\delta_{s_{i}^{l}, s_{j}^{l}}$ and $e_{s_{i}^{l}, s_{j}^{l}}$.

Define $\Theta$ and $E$ for batch policy

$$
\begin{aligned}
& \Theta=\left(\begin{array}{ccccccccccc}
\delta_{s_{1}^{l}, s_{n}^{l}} & 0 & \cdots & 0 & 0 & 0 & \cdots & 0 & 0 & \cdots & 0 \\
0 & \delta_{s_{1}^{l}, s_{1}^{l}} & \cdots & 0 & 0 & 0 & \cdots & 0 & 0 & \cdots & 0 \\
0 & 0 & \cdots & 0 & 0 & 0 & \cdots & 0 & 0 & \cdots & 0 \\
0 & 0 & \cdots & \delta_{s_{1}^{l}, s_{1}^{l}} & 0 & 0 & \cdots & 0 & 0 & \cdots & 0 \\
0 & 0 & \cdots & 0 & \delta_{s_{2}^{l}, s_{1}^{l}} & 0 & \cdots & 0 & 0 & \cdots & 0 \\
0 & 0 & \cdots & 0 & 0 & \delta_{s_{2}^{l}, s_{2}^{l}} & \cdots & 0 & 0 & \cdots & 0 \\
0 & 0 & \cdots & 0 & 0 & 0 & \cdots & 0 & 0 & \cdots & 0 \\
0 & 0 & \cdots & 0 & 0 & 0 & \cdots & \delta_{s_{n}^{l}, s_{n-1}^{l}} & 0 & \cdots & 0 \\
0 & 0 & \cdots & 0 & 0 & 0 & \cdots & 0 & \delta_{s_{n}^{l}, s_{n}^{l}} & \cdots & 0 \\
0 & 0 & \cdots & 0 & 0 & 0 & \cdots & 0 & 0 & \ddots & 0 \\
0 & 0 & \cdots & 0 & 0 & 0 & \cdots & 0 & 0 & \cdots & \delta_{s_{n}^{l}, s_{n}^{l}}
\end{array}\right), \\
& E=\left[e_{s_{1}^{l}, s_{n}^{l}}, e_{s_{1}^{l}, s_{1}^{l}}, \ldots, e_{s_{1}^{l}, s_{1}^{l}}, e_{s_{2}^{l}, s_{1}^{l}}, e_{s_{2}^{l}, s_{2}^{l}}, \ldots, e_{s_{n}^{l}, s_{n-1}^{l}}, e_{s_{n}^{l}, s_{n}^{l}}, \ldots, e_{s_{n}^{l}, s_{n}^{l}}\right]^{T} \text {, }
\end{aligned}
$$


and $\Theta$ and $E$ for strictly sequencing policy

$$
\begin{aligned}
\Theta & =\left(\begin{array}{cccc}
\delta_{s_{1}^{l}, s_{n}^{l}} & 0 & \cdots & 0 \\
0 & \delta_{s_{2}^{l}, s_{1}^{l}} & \cdots & 0 \\
0 & 0 & \ddots & 0 \\
0 & 0 & \cdots & \delta_{s_{n}^{l}, s_{n-1}^{l}}
\end{array}\right), \\
E & =\left[e_{s_{1}^{l}, s_{n}^{l}}, e_{s_{2}^{l}, s_{1}^{l}}, \ldots, e_{s_{n}^{l}, s_{n-1}^{l}}\right]^{T} .
\end{aligned}
$$

Then,

$$
\begin{aligned}
\Phi & =(I-\Theta) E, \\
\Theta & =\Gamma P .
\end{aligned}
$$

where

$$
P=\left(\begin{array}{cccc}
0 & 1 & \cdots & 0 \\
0 & 0 & \ddots & 0 \\
0 & 0 & \cdots & 1 \\
1 & 0 & \cdots & 0
\end{array}\right)
$$

Furthermore,

$$
\begin{aligned}
P\left(g^{l}\right) & =\operatorname{sum}\left(\frac{1}{K} \frac{\sum_{i=1}^{K} \Gamma^{i-1}}{\operatorname{det}(I-\Gamma)}(I-\Theta) E\right) \\
& =\operatorname{sum}\left(\frac{1}{K} \frac{\sum_{i=1}^{K} \Gamma^{i-1}}{\operatorname{det}(I-\Gamma)}(I-\Gamma P) E\right) \\
& =\operatorname{sum}\left(\frac{1}{K} \frac{\sum_{i=1}^{K} \Gamma^{i-1}}{\operatorname{det}(I-\Gamma)} E-\frac{1}{K} \frac{\sum_{i=1}^{K} \Gamma^{i}}{\operatorname{det}(I-\Gamma)} P E\right) \\
& =\operatorname{sum}\left(\frac{1}{K} \frac{I-\Gamma^{K} P}{\operatorname{det}(I-\Gamma)} E+\frac{1}{K} \frac{\sum_{i=2}^{K} \Gamma^{i-1}}{\operatorname{det}(I-\Gamma)} E-\frac{1}{K} \frac{\sum_{i=1}^{K-1} \Gamma^{i}}{\operatorname{det}(I-\Gamma)} P E\right) \\
& =\operatorname{sum}\left(\frac{1}{K} \frac{I-\Gamma^{K} P}{\operatorname{det}(I-\Gamma)} E\right)+\operatorname{sum}\left(\frac{1}{K} \frac{\sum_{i=1}^{K-1} \Gamma^{i}}{\operatorname{det}(I-\Gamma)}(I-P) E\right) .
\end{aligned}
$$

Since

$$
\operatorname{sum}\left(\left(I-\Gamma^{K} P\right) E\right)=\operatorname{det}(I-\Gamma) \operatorname{sum}(E),
$$


we can obtain

$$
\begin{aligned}
P\left(g^{l}\right) & =\operatorname{sum}\left(\frac{1}{K} E\right)+\operatorname{sum}\left(\frac{1}{K} \frac{\sum_{i=1}^{K-1} \Gamma^{i}}{\operatorname{det}(I-\Gamma)}(I-P) E\right) \\
& =\frac{1}{K} \operatorname{sum}(E)+\frac{1}{K \operatorname{det}(I-\Gamma)} \operatorname{sum}\left(\sum_{i=1}^{K-1} \Gamma^{i}(I-P) E\right) .
\end{aligned}
$$

Step 2: Partial derivatives and ignoring 2nd order $\delta_{s_{i}^{l}, s_{j}^{l}}$.

Let $\delta_{s_{i}^{l}, s_{j}^{l}}$ and $e_{s_{i}^{l}, s_{j}^{l}}$ for batch policy

$$
\begin{aligned}
& \delta_{s_{i}^{l}, s_{j}^{l}} \in\left\{\delta_{s_{1}^{l}, s_{n}^{l}}, \delta_{s_{2}^{l}, s_{1}^{l}}, \delta_{s_{3}^{l}, s_{2}^{l}}, \ldots, \delta_{s_{n}^{l}, s_{n-1}^{l}}, \delta_{s_{1}^{l}, s_{1}^{l}}, \delta_{s_{2}^{l}, s_{2}^{l}}, \ldots, \delta_{s_{n}^{l}, s_{n}^{l}}\right\}, \\
& e_{s_{i}^{l}, s_{j}^{l}} \in\left\{e_{s_{1}^{l}, s_{n}^{l}}, e_{s_{2}^{l}, s_{1}^{l}}, e_{s_{3}^{l}, s_{2}^{l}}, \ldots, e_{s_{n}^{l}, s_{n-1}^{l}}, e_{s_{1}^{l}, s_{1}^{l}}, e_{s_{2}^{l}, s_{2}^{l}}, \ldots, e_{s_{n}^{l}, s_{n}^{l}}\right\} .
\end{aligned}
$$

and $\delta_{s_{i}^{l}, s_{j}^{l}}$ and $e_{s_{i}^{l}, s_{j}^{l}}$ for strictly sequencing policy

$$
\begin{aligned}
& \delta_{s_{i}^{l}, s_{j}^{l}} \in\left\{\delta_{s_{1}^{l}, s_{n}^{l}}, \delta_{s_{2}^{l}, s_{1}^{l}}, \delta_{s_{3}^{l}, s_{2}^{l}}, \ldots, \delta_{s_{n}^{l}, s_{n-1}^{l}}\right\}, \\
& e_{s_{i}^{l}, s_{j}^{l}} \in\left\{e_{s_{1}^{l}, s_{n}^{l}}, e_{s_{2}^{l}, s_{1}^{l}}, e_{s_{3}^{l}, s_{2}^{l}}, \ldots, e_{s_{n}^{l}, s_{n-1}^{l}}\right\} .
\end{aligned}
$$

The partial derivatives can be calculated as

$$
\begin{aligned}
& \frac{\partial P\left(g^{l}\right)}{\partial \lambda_{s_{i}^{l}, s_{j}^{l}}}=\frac{\partial P\left(g^{l}\right)}{\partial \delta_{s_{i}^{l}, s_{j}^{l}}} \frac{\partial \delta_{s_{i}^{l}, s_{j}^{l}}}{\partial \lambda_{s_{i}^{l}, s_{j}^{l}}}+\frac{\partial P\left(g^{l}\right)}{\partial e_{s_{i}^{l}, s_{j}^{l}}} \frac{\partial e_{s_{i}^{l}, s_{j}^{l}}}{\partial \lambda_{s_{i}^{l}, s_{j}^{l}}}, \\
& \frac{\partial P\left(g^{l}\right)}{\partial \mu_{s_{i}^{l}, s_{j}^{l}}^{l}}=\frac{\partial P\left(g^{l}\right)}{\partial \delta_{s_{i}^{l}, s_{j}^{l}}} \frac{\partial \delta_{s_{i}^{l}, s_{j}^{l}}}{\partial \mu_{s_{i}^{l}, s_{j}^{l}}}+\frac{\partial P\left(g^{l}\right)}{\partial e_{s_{i}^{l}, s_{j}^{l}}} \frac{\partial e_{s_{i}^{l}, s_{j}^{l}}}{\partial \mu_{s_{i}^{l}, s_{j}^{l}}} .
\end{aligned}
$$

We can easily obtain

$$
\begin{aligned}
& \frac{\partial \delta_{s_{i}^{l}, s_{j}^{l}}}{\partial \lambda_{s_{i}^{l}, s_{j}^{l}}}=-1, \\
& \frac{\partial \delta_{s_{i}^{l}, s_{j}^{l}}}{\partial \mu_{s_{i}^{l}, s_{j}^{l}}}=-1 ; \\
& \frac{\partial e_{s_{i}^{l}, s_{j}^{l}}}{\partial \lambda_{s_{i}^{l}, s_{j}^{l}}}=-\frac{e_{s_{i}^{l}, s_{j}^{l}}}{1-\delta_{s_{i}^{l}, s_{j}^{l}}}, \\
& \frac{\partial e_{s_{i}^{l}, s_{j}^{l}}}{\partial \mu_{s_{i}^{l}, s_{j}^{l}}}=\frac{1-e_{s_{i}^{l}, s_{j}^{l}}}{1-\delta_{s_{i}^{l}, s_{j}^{l}}^{l}} .
\end{aligned}
$$


Then,

$$
\begin{aligned}
\frac{\partial P\left(g^{l}\right)}{\partial \delta_{s_{i}^{l}, s_{j}^{l}}=} & \frac{1}{K \operatorname{det}(I-\Gamma)} \frac{\partial \operatorname{sum}\left(\sum_{i=1}^{K-1} \Gamma^{i}(I-P) E\right)}{\partial \delta_{s_{i}^{l}, s_{j}^{l}}} \\
& -\frac{\operatorname{sum}\left(\sum_{i=1}^{K-1} \Gamma^{i}(I-P) E\right)}{K \operatorname{det}(I-\Gamma)^{2}} \frac{\partial \operatorname{det}(I-\Gamma)}{\partial \delta_{s_{i}^{l}, s_{j}^{l}}}, \\
\frac{\partial P\left(g^{l}\right)}{\partial e_{s_{i}^{l}, s_{j}^{l}}}= & \frac{1}{K} \frac{\partial \operatorname{sum}(E)}{\partial e_{s_{i}^{l}, s_{j}^{l}}}+\frac{1}{K \operatorname{det}(I-\Gamma)} \frac{\partial \operatorname{sum}\left(\sum_{i=1}^{K-1} \Gamma^{i}(I-P) E\right)}{\partial e_{s_{i}^{l}, s_{j}^{l}}} .
\end{aligned}
$$

\section{Strictly Sequencing Policy}

In this case, $i=j+1$ or $i=1$ when $j=n$. In the following derivation, the index $s_{i}^{l}, s_{j}^{l}$ must satisfy the rule:

if $i=1$ or $j=1$

$$
\begin{aligned}
& i-1=i-1+n, \\
& j-1=j-1+n .
\end{aligned}
$$

if $i=n$ or $j=n$

$$
\begin{aligned}
& i+1=i+1-n, \\
& j+1=j+1-n .
\end{aligned}
$$

Define the matrix $Q$

$$
Q=\left(\begin{array}{cccccc}
0 & \ldots & 0 & 0 & \ldots & 0 \\
0 & \ddots & 0 & 0 & \ddots & 0 \\
0 & \ldots & q_{i-1, i-1} & 0 & \ldots & 0 \\
0 & \ldots & 0 & q_{i, i} & \ldots & 0 \\
0 & \ddots & 0 & 0 & \ddots & 0 \\
0 & \ldots & 0 & 0 & \ldots & 0
\end{array}\right) .
$$

where $q_{i-1, i-1}=\operatorname{det}(I-\Gamma)$ and $q_{i, i}=1$, and $i-1=n$ if $i=1$. 
We can find

$$
\operatorname{det}(I-\Gamma) \frac{\partial \operatorname{sum}(E)}{\partial e_{s_{i}^{l}, s_{j}^{l}}}=\frac{\partial \operatorname{sum}(Q(I-P) E)}{\partial e_{s_{i}^{l}, s_{j}^{l}}}
$$

Considering

$$
\frac{\partial \operatorname{det}(I-\Gamma)}{\partial \delta_{s_{i}^{l}, s_{j}^{l}}}=o\left(\delta_{\max }^{n-1}\right)
$$

the partial derivatives can be obtained

$$
\begin{aligned}
& \frac{\partial P\left(g^{l}\right)}{\partial \lambda_{s_{i}^{l}, s_{j}^{l}}} \\
& =\frac{\partial P\left(g^{l}\right)}{\partial \delta_{s_{i}^{l}, s_{j}^{l}}} \frac{\partial \delta_{s_{i}^{l}, s_{j}^{l}}}{\partial \lambda_{s_{i}^{l}, s_{j}^{l}}}+\frac{\partial P\left(g^{l}\right)}{\partial e_{s_{i}^{l}, s_{j}^{l}}} \frac{\partial e_{s_{i}^{l}, s_{j}^{l}}}{\partial \lambda_{s_{i}^{l}, s_{j}^{l}}} \\
& =-\frac{1}{n \operatorname{det}(I-\Gamma)} \frac{\partial \operatorname{sum}\left(\sum_{i=1}^{n-1} \Gamma^{i}(I-P) E\right)}{\partial \delta_{s_{i}^{l}, s_{j}^{l}}}+\frac{o\left(\delta_{\max }^{n}\right)}{n \operatorname{det}(I-\Gamma)^{2}} \\
& -\frac{e_{s_{i}^{l}, s_{j}^{l}}}{1-\delta_{s_{i}^{l}, s_{j}^{l}}}\left[\frac{1}{n} \frac{\partial \operatorname{sum}(E)}{\partial e_{s_{i}^{l}, s_{j}^{l}}}+\frac{1}{n \operatorname{det}(I-\Gamma)} \frac{\partial \operatorname{sum}\left(\sum_{i=1}^{n-1} \Gamma^{i}(I-P) E\right)}{\partial e_{s_{i}^{l}, s_{j}^{l}}}\right] \\
& =-\frac{1}{n \operatorname{det}(I-\Gamma)}\left[\frac{\partial \operatorname{sum}\left(\sum_{i=1}^{2} \Gamma^{i}(I-P) E\right)}{\partial \delta_{s_{i}^{l}, s_{j}^{l}}}+o\left(\delta_{\max }^{2}\right)\right]+\frac{o\left(\delta_{\max }^{n}\right)}{n \operatorname{det}(I-\Gamma)^{2}} \\
& -\frac{1}{n \operatorname{det}(I-\Gamma)} \frac{e_{s_{i}^{l}, s_{j}^{l}}}{1-\delta_{s_{i}^{l}, s_{j}^{l}}}\left[\operatorname{det}(I-\Gamma) \frac{\partial \operatorname{sum}(E)}{\partial e_{s_{i}^{l}, s_{j}^{l}}}+\frac{\partial \operatorname{sum}\left(\sum_{i=1}^{n-1} \Gamma^{i}(I-P) E\right)}{\partial e_{s_{i}^{l}, s_{j}^{l}}}\right] \\
& =-\frac{1}{n \operatorname{det}(I-\Gamma)}\left[\frac{\partial \operatorname{sum}\left(\sum_{i=1}^{2} \Gamma^{i}(I-P) E\right)}{\partial \delta_{s_{i}^{l}, s_{j}^{l}}}\right]+\frac{o\left(\delta_{\max }^{2}\right)}{n \operatorname{det}(I-\Gamma)}+\frac{o\left(\delta_{\max }^{n}\right)}{n \operatorname{det}(I-\Gamma)^{2}} \\
& -\frac{1}{n \operatorname{det}(I-\Gamma)} \frac{e_{s_{i}^{l}, s_{j}^{l}}}{1-\delta_{s_{i}^{l}, s_{j}^{l}}}\left[\frac{\partial \operatorname{sum}\left(\left(Q+\sum_{i=1}^{n-1} \Gamma^{i}\right)(I-P) E\right)}{\partial e_{s_{i}^{l}, s_{j}^{l}}}\right] \\
& =-\frac{1}{n \operatorname{det}(I-\Gamma)}\left[\left(1+\delta_{s_{i+1}^{l}, s_{j+1}^{l}}\right)\left(e_{s_{i-1}^{l}, s_{j-1}^{l}}-e_{s_{i}^{l}, s_{j}^{l}}\right)\right. \\
& \left.+\delta_{s_{i-1}^{l}, s_{j-1}^{l}}\left(e_{s_{i-2}^{l}, s_{j-2}^{l}}-e_{s_{i-1}^{l}, s_{j-1}^{l}}\right)\right] \\
& -\frac{1}{n \operatorname{det}(I-\Gamma)} \frac{e_{s_{i}^{l}, s_{j}^{l}}}{1-\delta_{s_{i}^{l}, s_{j}^{l}}}\left[\left(1-\delta_{s_{i}^{l}, s_{j}^{l}}\right)\left(1+\delta_{s_{i+1}^{l}, s_{j+1}^{l}}+o\left(\delta_{\max }^{2}\right)\right)\right] \\
& +\frac{o\left(\delta_{\max }^{2}\right)}{n \operatorname{det}(I-\Gamma)}+\frac{o\left(\delta_{\max }^{n}\right)}{n \operatorname{det}(I-\Gamma)^{2}}
\end{aligned}
$$




$$
\begin{aligned}
= & -\frac{1}{n \operatorname{det}(I-\Gamma)}\left[\left(1+\delta_{s_{i+1}^{l}, s_{j+1}^{l}}\right) e_{s_{i-1}^{l}, s_{j-1}^{l}}+\delta_{s_{i-1}^{l}, s_{j-1}^{l}}\left(e_{s_{i-2}^{l}, s_{j-2}^{l}}-e_{s_{i-1}^{l}, s_{j-1}^{l}}\right)\right] \\
& +\frac{o\left(\delta_{\text {max }}^{2}\right)}{n \operatorname{det}(I-\Gamma)}+\frac{o\left(\delta_{\text {max }}^{n}\right)}{n \operatorname{det}(I-\Gamma)^{2}} \\
= & -\frac{1}{n\left(1-o\left(\delta_{\text {max }}^{n}\right)\right)}\left[\left(1+\delta_{s_{i+1}^{l}, s_{j+1}^{l}}\right) e_{s_{i-1}^{l}, s_{j-1}^{l}}+\delta_{s_{i-1}^{l}, s_{j-1}^{l}}\left(e_{s_{i-2}^{l}, s_{j-2}^{l}}-e_{s_{i-1}^{l}, s_{j-1}^{l}}\right)\right] \\
& +\frac{o\left(\delta_{\text {max }}^{2}\right)}{n\left(1-o\left(\delta_{\text {max }}^{n}\right)\right)}+\frac{o\left(\delta_{\max }^{n}\right)}{n\left(1-o\left(\delta_{\max }^{n}\right)\right)} \\
= & -\frac{1}{n}\left[\left(1+\delta_{s_{i+1}^{l}, s_{j+1}^{l}}\right) e_{s_{i-1}^{l}, s_{j-1}^{l}}+\delta_{s_{i-1}^{l}, s_{j-1}^{l}}\left(e_{s_{i-2}^{l}, s_{j-2}^{l}}-e_{s_{i-1}^{l}, s_{j-1}^{l}}\right)\right]+o\left(\delta_{\text {max }}^{2}\right) .
\end{aligned}
$$

$$
\begin{aligned}
& \frac{\partial P\left(g^{l}\right)}{\partial \mu_{s_{i}^{l}, s_{j}^{l}}} \\
& =\frac{\partial P\left(g^{l}\right)}{\partial \delta_{s_{i}^{l}, s_{j}^{l}}} \frac{\partial \delta_{s_{i}^{l}, s_{j}^{l}}}{\partial \mu_{s_{i}^{l}, s_{j}^{l}}}+\frac{\partial P\left(g^{l}\right)}{\partial e_{s_{i}^{l}, s_{j}^{l}}} \frac{\partial e_{s_{i}^{l}, s_{j}^{l}}}{\partial \mu_{s_{i}^{l}, s_{j}^{l}}} \\
& =-\frac{1}{n \operatorname{det}(I-\Gamma)} \frac{\partial \operatorname{sum}\left(\sum_{i=1}^{n-1} \Gamma^{i}(I-P) E\right)}{\partial \delta_{s_{i}^{l}, s_{j}^{l}}}+\frac{o\left(\delta_{\max }^{n}\right)}{n \operatorname{det}(I-\Gamma)^{2}} \\
& +\frac{1-e_{s_{i}^{l}, s_{j}^{l}}}{1-\delta_{s_{i}^{l}, s_{j}^{l}}}\left[\frac{1}{n} \frac{\partial \operatorname{sum}(E)}{\partial e_{s_{i}^{l}, s_{j}^{l}}}+\frac{1}{n \operatorname{det}(I-\Gamma)} \frac{\partial \operatorname{sum}\left(\sum_{i=1}^{n-1} \Gamma^{i}(I-P) E\right)}{\partial e_{s_{i}^{l}, s_{j}^{l}}}\right] \\
& =-\frac{1}{n \operatorname{det}(I-\Gamma)}\left[\frac{\partial \operatorname{sum}\left(\sum_{i=1}^{2} \Gamma^{i}(I-P) E\right)}{\partial \delta_{s_{i}^{l}, s_{j}^{l}}}+o\left(\delta_{\text {max }}^{2}\right)\right]+\frac{o\left(\delta_{\max }^{n}\right)}{n \operatorname{det}(I-\Gamma)^{2}} \\
& +\frac{1}{n \operatorname{det}(I-\Gamma)} \frac{1-e_{s_{i}^{l}, s_{j}^{l}}}{1-\delta_{s_{i}^{l}, s_{j}^{l}}}\left[\operatorname{det}(I-\Gamma) \frac{\partial \operatorname{sum}(E)}{\partial e_{s_{i}^{l}, s_{j}^{l}}}+\frac{\partial \operatorname{sum}\left(\sum_{i=1}^{n-1} \Gamma^{i}(I-P) E\right)}{\partial e_{s_{i}^{l}, s_{j}^{l}}}\right] \\
& =-\frac{1}{n \operatorname{det}(I-\Gamma)}\left[\frac{\partial \operatorname{sum}\left(\sum_{i=1}^{2} \Gamma^{i}(I-P) E\right)}{\partial \delta_{s_{i}^{l}, s_{j}^{l}}}\right]+\frac{o\left(\delta_{\max }^{2}\right)}{n \operatorname{det}(I-\Gamma)}+\frac{o\left(\delta_{\max }^{n}\right)}{n \operatorname{det}(I-\Gamma)^{2}} \\
& +\frac{1}{n \operatorname{det}(I-\Gamma)} \frac{1-e_{s_{i}^{l}, s_{j}^{l}}}{1-\delta_{s_{i}^{l}, s_{j}^{l}}}\left[\frac{\partial \operatorname{sum}\left(\left(Q+\sum_{i=1}^{n-1} \Gamma^{i}\right)(I-P) E\right)}{\partial e_{s_{i}^{l}, s_{j}^{l}}}\right] \\
& =-\frac{1}{n \operatorname{det}(I-\Gamma)}\left[\left(1+\delta_{s_{i+1}^{l}, s_{j+1}^{l}}\right)\left(e_{s_{i-1}^{l}, s_{j-1}^{l}}-e_{i, j}\right)\right. \\
& \left.+\delta_{s_{i-1}^{l}, s_{j-1}^{l}}\left(e_{s_{i-2}^{l}, s_{j-2}^{l}}-e_{s_{i-1}^{l}, s_{j-1}^{l}}\right)\right] \\
& +\frac{1}{n \operatorname{det}(I-\Gamma)} \frac{1-e_{s_{i}^{l}, s_{j}^{l}}}{1-\delta_{s_{i}^{l}, s_{j}^{l}}}\left[\left(1-\delta_{s_{i}^{l}, s_{j}^{l}}\right)\left(1+\delta_{s_{i+1}^{l}, s_{j+1}^{l}}+o\left(\delta_{\text {max }}^{2}\right)\right)\right] \\
& +\frac{o\left(\delta_{\max }^{2}\right)}{n \operatorname{det}(I-\Gamma)}+\frac{o\left(\delta_{\max }^{n}\right)}{n \operatorname{det}(I-\Gamma)^{2}}
\end{aligned}
$$




$$
\begin{aligned}
= & -\frac{1}{n \operatorname{det}(I-\Gamma)}\left[\left(1+\delta_{s_{i+1}^{l}, s_{j+1}^{l}}\right)\left(e_{s_{i-1}^{l}, s_{j-1}^{l}}-1\right)+\delta_{s_{i-1}^{l}, s_{j-1}^{l}}\left(e_{s_{i-2}^{l}, s_{j-2}^{l}}-e_{s_{i-1}^{l}, s_{j-1}^{l}}\right)\right] \\
& +\frac{o\left(\delta_{\max }^{2}\right)}{n \operatorname{det}(I-\Gamma)}+\frac{o\left(\delta_{\max }^{n}\right)}{n \operatorname{det}(I-\Gamma)^{2}} \\
= & -\frac{1}{n\left(1-o\left(\delta_{\text {max }}^{n}\right)\right)}\left[\left(1+\delta_{s_{i+1}^{l}, s_{j+1}^{l}}\right)\left(e_{s_{i-1}^{l}, s_{j-1}^{l}}-1\right)+\delta_{s_{i-1}^{l}, s_{j-1}^{l}}\left(e_{s_{i-2}^{l}, s_{j-2}^{l}}-e_{s_{i-1}^{l}, s_{j-1}^{l}}\right)\right] \\
& +\frac{o\left(\delta_{\max }^{2}\right)}{n\left(1-o\left(\delta_{\text {max }}^{n}\right)\right)}+\frac{o\left(\delta_{\max }^{n}\right)}{n\left(1-o\left(\delta_{\max }^{n}\right)\right)} \\
= & -\frac{1}{n}\left[\left(1+\delta_{s_{i+1}^{l}, s_{j+1}^{l}}^{l}\right)\left(e_{s_{i-1}^{l}, s_{j-1}^{l}}-1\right)+\delta_{s_{i-1}^{l}, s_{j-1}^{l}}\left(e_{s_{i-2}^{l}, s_{j-2}^{l}}-e_{s_{i-1}^{l}, s_{j-1}^{l}}^{l}\right)\right]+o\left(\delta_{\text {max }}^{2}\right) .
\end{aligned}
$$

\section{Batch Policy}

In this case, $i=j+1$ or $i=1$ when $j=n$ for transitions with switch, and $i=j$ for

transitions without switch. In the following derivation, the index $s_{i}^{l}, s_{j}^{l}$ must satisfy the rule:

if $i=1$ or $j=1$

$$
\begin{aligned}
& i-1=i-1+n, \\
& j-1=j-1+n .
\end{aligned}
$$

if $i=n$ or $j=n$

$$
\begin{aligned}
& i+1=i+1-n, \\
& j+1=j+1-n .
\end{aligned}
$$

\section{Transitions with switch:}

Define the matrix $Q$

$$
Q=\left(\begin{array}{cccccc}
0 & \ldots & 0 & 0 & \ldots & 0 \\
0 & \ddots & 0 & 0 & \ddots & 0 \\
0 & \ldots & q_{m-1, m-1} & 0 & \ldots & 0 \\
0 & \ldots & 0 & q_{m, m} & \ldots & 0 \\
0 & \ddots & 0 & 0 & \ddots & 0 \\
0 & \ldots & 0 & 0 & \ldots & 0
\end{array}\right)
$$


where $m=\sum_{h=2}^{i}\left(k_{s_{h-1}^{l}}+1\right)$ when $i>1$ or $m=1$ when $i=1, q_{m-1, m-1}=\operatorname{det}(I-\Gamma)$ and $q_{m, m}=1$, and $m-1=K$ if $m=1$.

We can find

$$
\operatorname{det}(I-\Gamma) \frac{\partial \operatorname{sum}(E)}{\partial e_{s_{i}^{l}, s_{j}^{l}}}=\frac{\partial \operatorname{sum}(Q(I-P) E)}{\partial e_{s_{i}^{l}, s_{j}^{l}}} .
$$

Considering

$$
\frac{\partial \operatorname{det}(I-\Gamma)}{\partial \delta_{s_{i}^{l}, s_{j}^{l}}^{l}}=o\left(\delta_{\max }^{n-1}\right)
$$

the partial derivatives can be obtained

If the batch size $k_{s_{j}^{l}}=2$

$$
\begin{aligned}
& \frac{\partial P\left(g^{l}\right)}{\partial \lambda_{s_{i}^{l}, s_{j}^{l}}} \\
& =\frac{\partial P\left(g^{l}\right)}{\partial \delta_{s_{i}^{l}, s_{j}^{l}}} \frac{\partial \delta_{s_{i}^{l}, s_{j}^{l}}}{\partial \lambda_{s_{i}^{l}, s_{j}^{l}}}+\frac{\partial P\left(g^{l}\right)}{\partial e_{s_{i}^{l}, s_{j}^{l}}} \frac{\partial e_{s_{i}^{l}, s_{j}^{l}}}{\partial \lambda_{s_{i}^{l}, s_{j}^{l}}} \\
& =-\frac{1}{K \operatorname{det}(I-\Gamma)} \frac{\partial \operatorname{sum}\left(\sum_{i=1}^{K-1} \Gamma^{i}(I-P) E\right)}{\partial \delta_{s_{i}^{l}, s_{j}^{l}}^{l}}+\frac{o\left(\delta_{\max }^{K}\right)}{K \operatorname{det}(I-\Gamma)^{2}} \\
& -\frac{e_{s_{i}^{l}, s_{j}^{l}}}{1-\delta_{s_{i}^{l}, s_{j}^{l}}}\left[\frac{1}{K} \frac{\partial \operatorname{sum}(E)}{\partial e_{s_{i}^{l}, s_{j}^{l}}}+\frac{1}{K \operatorname{det}(I-\Gamma)} \frac{\partial \operatorname{sum}\left(\sum_{i=1}^{K-1} \Gamma^{i}(I-P) E\right)}{\partial e_{s_{i}^{l}, s_{j}^{l}}}\right] \\
& =-\frac{1}{K \operatorname{det}(I-\Gamma)}\left[\frac{\partial \operatorname{sum}\left(\sum_{i=1}^{2} \Gamma^{i}(I-P) E\right)}{\partial \delta_{s_{i}^{l}, s_{j}^{l}}}+o\left(\delta_{\max }^{2}\right)\right]+\frac{o\left(\delta_{\max }^{K}\right)}{K \operatorname{det}(I-\Gamma)^{2}} \\
& -\frac{1}{K \operatorname{det}(I-\Gamma)} \frac{e_{s_{i}^{l}, s_{j}^{l}}}{1-\delta_{s_{i}^{l}, s_{j}^{l}}}\left[\operatorname{det}(I-\Gamma) \frac{\partial \operatorname{sum}(E)}{\partial e_{s_{i}^{l}, s_{j}^{l}}}+\frac{\partial \operatorname{sum}\left(\sum_{i=1}^{K-1} \Gamma^{i}(I-P) E\right)}{\partial e_{s_{i}^{l}, s_{j}^{l}}}\right] \\
& =-\frac{1}{K \operatorname{det}(I-\Gamma)}\left[\frac{\partial \operatorname{sum}\left(\sum_{i=1}^{2} \Gamma^{i}(I-P) E\right)}{\partial \delta_{s_{i}^{l}, s_{j}^{l}}}\right]+\frac{o\left(\delta_{\max }^{2}\right)}{K \operatorname{det}(I-\Gamma)}+\frac{o\left(\delta_{\max }^{K}\right)}{K \operatorname{det}(I-\Gamma)^{2}} \\
& -\frac{1}{K \operatorname{det}(I-\Gamma)} \frac{e_{s_{i}^{l}, s_{j}^{l}}}{1-\delta_{s_{i}^{l}, s_{j}^{l}}}\left[\frac{\partial \operatorname{sum}\left(\left(Q+\sum_{i=1}^{K-1} \Gamma^{i}\right)(I-P) E\right)}{\partial e_{s_{i}^{l}, s_{j}^{l}}}\right] \\
& =-\frac{1}{K \operatorname{det}(I-\Gamma)}\left[\left(1+\delta_{s_{i}^{l}, s_{j+1}^{l}}\right)\left(e_{s_{i-1}^{l}, s_{j}^{l}}-e_{s_{i}^{l}, s_{j}^{l}}\right)+\delta_{s_{i-1}^{l}, s_{j}^{l}}\left(e_{s_{i-1}^{l}, s_{j-1}^{l}}-e_{s_{i-1}^{l}, s_{j}^{l}}\right)\right]
\end{aligned}
$$




$$
\begin{aligned}
& -\frac{1}{K \operatorname{det}(I-\Gamma)} \frac{e_{s_{i}^{l}, s_{j}^{l}}}{1-\delta_{s_{i}^{l}, s_{j}^{l}}}\left[\left(1-\delta_{s_{i}^{l}, s_{j}^{l}}\right)\left(1+\delta_{s_{i}^{l}, s_{j+1}^{l}}+o\left(\delta_{\max }^{2}\right)\right)\right] \\
& +\frac{o\left(\delta_{\max }^{2}\right)}{K \operatorname{det}(I-\Gamma)}+\frac{o\left(\delta_{\max }^{K}\right)}{K \operatorname{det}(I-\Gamma)^{2}} \\
& =-\frac{1}{K \operatorname{det}(I-\Gamma)}\left[\left(1+\delta_{s_{i}^{l}, s_{j+1}^{l}}\right) e_{s_{i-1}^{l}, s_{j}^{l}}+\delta_{s_{i-1}^{l}, s_{j}^{l}}\left(e_{s_{i-1}^{l}, s_{j-1}^{l}}-e_{s_{i-1}^{l}, s_{j}^{l}}\right)\right] \\
& +\frac{o\left(\delta_{\max }^{2}\right)}{K \operatorname{det}(I-\Gamma)}+\frac{o\left(\delta_{\max }^{K}\right)}{K \operatorname{det}(I-\Gamma)^{2}} \\
& =-\frac{1}{K\left(1-o\left(\delta_{\max }^{K}\right)\right)}\left[\left(1+\delta_{s_{i}^{l}, s_{j+1}^{l}}\right) e_{s_{i-1}^{l}, s_{j}^{l}}+\delta_{s_{i-1}^{l}, s_{j}^{l}}\left(e_{s_{i-1}^{l}, s_{j-1}^{l}}-e_{s_{i-1}^{l}, s_{j}^{l}}\right)\right] \\
& +\frac{o\left(\delta_{\max }^{2}\right)}{K\left(1-o\left(\delta_{\max }^{K}\right)\right)}+\frac{o\left(\delta_{\max }^{K}\right)}{K\left(1-o\left(\delta_{\max }^{K}\right)\right)} \\
& =-\frac{1}{K}\left[\left(1+\delta_{s_{i}^{l}, s_{j+1}^{l}}\right) e_{s_{i-1}^{l}, s_{j}^{l}}+\delta_{s_{i-1}^{l}, s_{j}^{l}}\left(e_{s_{i-1}^{l}, s_{j-1}^{l}}-e_{s_{i-1}^{l}, s_{j}^{l}}\right)\right]+o\left(\delta_{\text {max }}^{2}\right) \text {. } \\
& \frac{\partial P\left(g^{l}\right)}{\partial \mu_{s_{i}^{l}, s_{j}^{l}}} \\
& =\frac{\partial P\left(g^{l}\right)}{\partial \delta_{s_{i}^{l}, s_{j}^{l}}} \frac{\partial \delta_{s_{i}^{l}, s_{j}^{l}}}{\partial \mu_{s_{i}^{l}, s_{j}^{l}}}+\frac{\partial P\left(g^{l}\right)}{\partial e_{s_{i}^{l}, s_{j}^{l}}^{l}} \frac{\partial e_{s_{i}^{l}, s_{j}^{l}}}{\partial \mu_{s_{i}^{l}, s_{j}^{l}}} \\
& =-\frac{1}{K \operatorname{det}(I-\Gamma)} \frac{\partial \operatorname{sum}\left(\sum_{i=1}^{K-1} \Gamma^{i}(I-P) E\right)}{\partial \delta_{s_{i}^{l}, s_{j}^{l}}^{l}}+\frac{o\left(\delta_{\max }^{K}\right)}{K \operatorname{det}(I-\Gamma)^{2}} \\
& +\frac{1-e_{s_{i}^{l}, s_{j}^{l}}}{1-\delta_{s_{i}^{l}, s_{j}^{l}}}\left[\frac{1}{K} \frac{\partial \operatorname{sum}(E)}{\partial e_{s_{i}^{l}, s_{j}^{l}}}+\frac{1}{K \operatorname{det}(I-\Gamma)} \frac{\partial \operatorname{sum}\left(\sum_{i=1}^{K-1} \Gamma^{i}(I-P) E\right)}{\partial e_{s_{i}^{l}, s_{j}^{l}}}\right] \\
& =-\frac{1}{K \operatorname{det}(I-\Gamma)}\left[\frac{\partial \operatorname{sum}\left(\sum_{i=1}^{2} \Gamma^{i}(I-P) E\right)}{\partial \delta_{s_{i}^{l}, s_{j}^{l}}^{l}}+o\left(\delta_{\max }^{2}\right)\right]+\frac{o\left(\delta_{\max }^{K}\right)}{K \operatorname{det}(I-\Gamma)^{2}} \\
& +\frac{1}{K \operatorname{det}(I-\Gamma)} \frac{1-e_{s_{i}^{l}, s_{j}^{l}}}{1-\delta_{s_{i}^{l}, s_{j}^{l}}}\left[\operatorname{det}(I-\Gamma) \frac{\partial \operatorname{sum}(E)}{\partial e_{s_{i}^{l}, s_{j}^{l}}}+\frac{\partial \operatorname{sum}\left(\sum_{i=1}^{K-1} \Gamma^{i}(I-P) E\right)}{\partial e_{s_{i}^{l}, s_{j}^{l}}}\right] \\
& =-\frac{1}{K \operatorname{det}(I-\Gamma)}\left[\frac{\partial \operatorname{sum}\left(\sum_{i=1}^{2} \Gamma^{i}(I-P) E\right)}{\partial \delta_{s_{i}^{l}, s_{j}^{l}}}\right]+\frac{o\left(\delta_{\max }^{2}\right)}{K \operatorname{det}(I-\Gamma)}+\frac{o\left(\delta_{\max }^{K}\right)}{K \operatorname{det}(I-\Gamma)^{2}} \\
& +\frac{1}{K \operatorname{det}(I-\Gamma)} \frac{1-e_{s_{i}^{l}, s_{j}^{l}}}{1-\delta_{s_{i}^{l}, s_{j}^{l}}}\left[\frac{\partial \operatorname{sum}\left(\left(Q+\sum_{i=1}^{K-1} \Gamma^{i}\right)(I-P) E\right)}{\partial e_{s_{i}^{l}, s_{j}^{l}}}\right] \\
& =-\frac{1}{K \operatorname{det}(I-\Gamma)}\left[\left(1+\delta_{s_{i}^{l}, s_{j+1}^{l}}\right)\left(e_{s_{i-1}^{l}, s_{j}^{l}}-e_{s_{i}^{l}, s_{j}}\right)+\delta_{s_{i-1}^{l}, s_{j}^{l}}\left(e_{s_{i-1}^{l}, s_{j-1}^{l}}-e_{s_{i-1}^{l}, s_{j}^{l}}\right)\right] \\
& +\frac{1}{K \operatorname{det}(I-\Gamma)} \frac{1-e_{s_{i}^{l}, s_{j}^{l}}}{1-\delta_{s_{i}^{l}, s_{j}^{l}}^{l}}\left[\left(1-\delta_{s_{i}^{l}, s_{j}^{l}}\right)\left(1+\delta_{s_{i}^{l}, s_{j+1}^{l}}+o\left(\delta_{\max }^{2}\right)\right)\right]
\end{aligned}
$$




$$
\begin{aligned}
& +\frac{o\left(\delta_{\max }^{2}\right)}{K \operatorname{det}(I-\Gamma)}+\frac{o\left(\delta_{\max }^{K}\right)}{K \operatorname{det}(I-\Gamma)^{2}} \\
= & -\frac{1}{K \operatorname{det}(I-\Gamma)}\left[\left(1+\delta_{s_{i}^{l}, s_{j+1}^{l}}\right)\left(e_{s_{i-1}^{l}, s_{j}^{l}}-1\right)+\delta_{s_{i-1}^{l}, s_{j}^{l}}\left(e_{s_{i-1}^{l}, s_{j-1}^{l}}-e_{s_{i-1}^{l}, s_{j}^{l}}\right)\right] \\
& +\frac{o\left(\delta_{\max }^{2}\right)}{K \operatorname{det}(I-\Gamma)}+\frac{o\left(\delta_{\max }^{K}\right)}{K \operatorname{det}(I-\Gamma)^{2}} \\
= & -\frac{1}{K\left(1-o\left(\delta_{\max }^{K}\right)\right)}\left[\left(1+\delta_{s_{i}^{l}, s_{j+1}^{l}}\right)\left(e_{s_{i-1}^{l}, s_{j}^{l}}-1\right)+\delta_{s_{i-1}^{l}, s_{j}^{l}}\left(e_{s_{i-1}^{l}, s_{j-1}^{l}}-e_{s_{i-1}^{l}, s_{j}^{l}}\right)\right] \\
& +\frac{o\left(\delta_{\max }^{2}\right)}{K\left(1-o\left(\delta_{\max }^{K}\right)\right)}+\frac{o\left(\delta_{\max }^{K}\right)}{K\left(1-o\left(\delta_{\max }^{K}\right)\right)} \\
= & -\frac{1}{K}\left[\left(1+\delta_{s_{i}^{l}, s_{j+1}^{l}}\right)\left(e_{s_{i-1}^{l}, s_{j}^{l}}-1\right)+\delta_{s_{i-1}^{l}, s_{j}^{l}}\left(e_{s_{i-1}^{l}, s_{j-1}^{l}}-e_{s_{i-1}^{l}, s_{j}^{l}}\right)\right]+o\left(\delta_{\max }^{2}\right) .
\end{aligned}
$$

If the batch size $k_{s_{j}^{l}}>2$

$$
\begin{aligned}
& \frac{\partial P\left(g^{l}\right)}{\partial \lambda_{s_{i}^{l}, s_{j}^{l}}} \\
& =\frac{\partial P\left(g^{l}\right)}{\partial \delta_{s_{i}^{l}, s_{j}^{l}}} \frac{\partial \delta_{s_{i}^{l}, s_{j}^{l}}}{\partial \lambda_{s_{i}^{l}, s_{j}^{l}}}+\frac{\partial P\left(g^{l}\right)}{\partial e_{s_{i}^{l}, s_{j}^{l}}} \frac{\partial e_{s_{i}^{l}, s_{j}^{l}}}{\partial \lambda_{s_{i}^{l}, s_{j}^{l}}} \\
& =-\frac{1}{K \operatorname{det}(I-\Gamma)} \frac{\partial \operatorname{sum}\left(\sum_{i=1}^{K-1} \Gamma^{i}(I-P) E\right)}{\partial \delta_{s_{i}^{l}, s_{j}^{l}}}+\frac{o\left(\delta_{\max }^{K}\right)}{K \operatorname{det}(I-\Gamma)^{2}} \\
& -\frac{e_{s_{i}^{l}, s_{j}^{l}}}{1-\delta_{s_{i}^{l}, s_{j}^{l}}}\left[\frac{1}{K} \frac{\partial \operatorname{sum}(E)}{\partial e_{s_{i}^{l}, s_{j}^{l}}}+\frac{1}{K \operatorname{det}(I-\Gamma)} \frac{\partial \operatorname{sum}\left(\sum_{i=1}^{K-1} \Gamma^{i}(I-P) E\right)}{\partial e_{s_{i}^{l}, s_{j}^{l}}}\right] \\
& =-\frac{1}{K \operatorname{det}(I-\Gamma)}\left[\frac{\partial \operatorname{sum}\left(\sum_{i=1}^{2} \Gamma^{i}(I-P) E\right)}{\partial \delta_{s_{i}^{l}, s_{j}^{l}}}+o\left(\delta_{\max }^{2}\right)\right]+\frac{o\left(\delta_{\max }^{K}\right)}{K \operatorname{det}(I-\Gamma)^{2}} \\
& -\frac{1}{K \operatorname{det}(I-\Gamma)} \frac{e_{s_{i}^{l}, s_{j}^{l}}}{1-\delta_{s_{i}^{l}, s_{j}^{l}}}\left[\operatorname{det}(I-\Gamma) \frac{\partial \operatorname{sum}(E)}{\partial e_{s_{i}^{l}, s_{j}^{l}}}+\frac{\partial \operatorname{sum}\left(\sum_{i=1}^{K-1} \Gamma^{i}(I-P) E\right)}{\partial e_{s_{i}^{l}, s_{j}^{l}}}\right] \\
& =-\frac{1}{K \operatorname{det}(I-\Gamma)}\left[\frac{\partial \operatorname{sum}\left(\sum_{i=1}^{2} \Gamma^{i}(I-P) E\right)}{\partial \delta_{s_{i}^{l}, s_{j}^{l}}}\right]+\frac{o\left(\delta_{\max }^{2}\right)}{K \operatorname{det}(I-\Gamma)}+\frac{o\left(\delta_{\max }^{K}\right)}{K \operatorname{det}(I-\Gamma)^{2}} \\
& -\frac{1}{K \operatorname{det}(I-\Gamma)} \frac{e_{s_{i}^{l}, s_{j}^{l}}}{1-\delta_{s_{i}^{l}, s_{j}^{l}}}\left[\frac{\partial \operatorname{sum}\left(\left(Q+\sum_{i=1}^{K-1} \Gamma^{i}\right)(I-P) E\right)}{\partial e_{s_{i}^{l}, s_{j}^{l}}}\right] \\
& =-\frac{1}{K \operatorname{det}(I-\Gamma)}\left[\left(1+\delta_{s_{i}^{l}, s_{j+1}^{l}}\right)\left(e_{s_{i-1}^{l}, s_{j}^{l}}-e_{s_{i}^{l}, s_{j}^{l}}\right)+\delta_{s_{i-1}^{l}, s_{j}^{l}}\left(e_{s_{i-1}^{l}, s_{j}^{l}}-e_{s_{i-1}^{l}, s_{j}^{l}}\right)\right] \\
& -\frac{1}{K \operatorname{det}(I-\Gamma)} \frac{e_{s_{i}^{l}, s_{j}^{l}}}{1-\delta_{s_{i}^{l}, s_{j}^{l}}}\left[\left(1-\delta_{s_{i}^{l}, s_{j}^{l}}\right)\left(1+\delta_{s_{i}^{l}, s_{j+1}^{l}}+o\left(\delta_{\text {max }}^{2}\right)\right)\right]
\end{aligned}
$$




$$
\begin{aligned}
& +\frac{o\left(\delta_{\max }^{2}\right)}{K \operatorname{det}(I-\Gamma)}+\frac{o\left(\delta_{\max }^{K}\right)}{K \operatorname{det}(I-\Gamma)^{2}} \\
& =-\frac{1}{K \operatorname{det}(I-\Gamma)}\left[\left(1+\delta_{s_{i}^{l}, s_{j+1}^{l}}\right) e_{s_{i-1}^{l}, s_{j}^{l}}\right]+\frac{o\left(\delta_{\max }^{2}\right)}{K \operatorname{det}(I-\Gamma)}+\frac{o\left(\delta_{\max }^{K}\right)}{K \operatorname{det}(I-\Gamma)^{2}} \\
& =-\frac{1}{K\left(1-o\left(\delta_{\max }^{K}\right)\right)}\left[\left(1+\delta_{s_{i}^{l}, s_{j+1}^{l}}\right) e_{s_{i-1}^{l}, s_{j}^{l}}\right]+\frac{o\left(\delta_{\max }^{2}\right)}{K\left(1-o\left(\delta_{\max }^{K}\right)\right)}+\frac{o\left(\delta_{\max }^{K}\right)}{K\left(1-o\left(\delta_{\max }^{K}\right)\right)} \\
& =-\frac{1}{K}\left[\left(1+\delta_{s_{i}^{l}, s_{j+1}^{l}}\right) e_{s_{i-1}^{l}, s_{j}^{l}}\right]+o\left(\delta_{\text {max }}^{2}\right) \text {. } \\
& \frac{\partial P\left(g^{l}\right)}{\partial \mu_{s_{i}^{l}, s_{j}^{l}}} \\
& =\frac{\partial P\left(g^{l}\right)}{\partial \delta_{s_{i}^{l}, s_{j}^{l}}} \frac{\partial \delta_{s_{i}^{l}, s_{j}^{l}}}{\partial \mu_{s_{i}^{l}, s_{j}^{l}}}+\frac{\partial P\left(g^{l}\right)}{\partial e_{s_{i}^{l}, s_{j}^{l}}} \frac{\partial e_{s_{i}^{l}, s_{j}^{l}}}{\partial \mu_{s_{i}^{l}, s_{j}^{l}}} \\
& =-\frac{1}{K \operatorname{det}(I-\Gamma)} \frac{\partial \operatorname{sum}\left(\sum_{i=1}^{K-1} \Gamma^{i}(I-P) E\right)}{\partial \delta_{s_{i}^{l}, s_{j}^{l}}}+\frac{o\left(\delta_{\max }^{K}\right)}{K \operatorname{det}(I-\Gamma)^{2}} \\
& +\frac{1-e_{s_{i}^{l}, s_{j}^{l}}}{1-\delta_{s_{i}^{l}, s_{j}^{l}}}\left[\frac{1}{K} \frac{\partial \operatorname{sum}(E)}{\partial e_{s_{i}^{l}, s_{j}^{l}}}+\frac{1}{K \operatorname{det}(I-\Gamma)} \frac{\partial \operatorname{sum}\left(\sum_{i=1}^{K-1} \Gamma^{i}(I-P) E\right)}{\partial e_{s_{i}^{l}, s_{j}^{l}}}\right] \\
& =-\frac{1}{K \operatorname{det}(I-\Gamma)}\left[\frac{\partial \operatorname{sum}\left(\sum_{i=1}^{2} \Gamma^{i}(I-P) E\right)}{\partial \delta_{s_{i}^{l}, s_{j}^{l}}}+o\left(\delta_{\max }^{2}\right)\right]+\frac{o\left(\delta_{\max }^{K}\right)}{K \operatorname{det}(I-\Gamma)^{2}} \\
& +\frac{1}{K \operatorname{det}(I-\Gamma)} \frac{1-e_{s_{i}^{l}, s_{j}^{l}}}{1-\delta_{s_{i}^{l}, s_{j}^{l}}}\left[\operatorname{det}(I-\Gamma) \frac{\partial \operatorname{sum}(E)}{\partial e_{s_{i}^{l}, s_{j}^{l}}}+\frac{\partial \operatorname{sum}\left(\sum_{i=1}^{K-1} \Gamma^{i}(I-P) E\right)}{\partial e_{s_{i}^{l}, s_{j}^{l}}}\right] \\
& =-\frac{1}{K \operatorname{det}(I-\Gamma)}\left[\frac{\partial \operatorname{sum}\left(\sum_{i=1}^{2} \Gamma^{i}(I-P) E\right)}{\partial \delta_{s_{i}^{l}, s_{j}^{l}}}\right]+\frac{o\left(\delta_{\max }^{2}\right)}{K \operatorname{det}(I-\Gamma)}+\frac{o\left(\delta_{\max }^{K}\right)}{K \operatorname{det}(I-\Gamma)^{2}} \\
& +\frac{1}{K \operatorname{det}(I-\Gamma)} \frac{1-e_{s_{i}^{l}, s_{j}^{l}}}{1-\delta_{s_{i}^{l}, s_{j}^{l}}}\left[\frac{\partial \operatorname{sum}\left(\left(Q+\sum_{i=1}^{K-1} \Gamma^{i}\right)(I-P) E\right)}{\partial e_{s_{i}^{l}, s_{j}^{l}}}\right] \\
& =-\frac{1}{K \operatorname{det}(I-\Gamma)}\left[\left(1+\delta_{s_{i}^{l}, s_{j+1}^{l}}\right)\left(e_{s_{i-1}^{l}, s_{j}^{l}}-e_{s_{i}^{l}, s_{j}^{l}}\right)+\delta_{s_{i-1}^{l}, s_{j}^{l}}\left(e_{s_{i-1}^{l}, s_{j}^{l}}-e_{s_{i-1}^{l}, s_{j}^{l}}\right)\right] \\
& +\frac{1}{K \operatorname{det}(I-\Gamma)} \frac{1-e_{s_{i}^{l}, s_{j}^{l}}}{1-\delta_{s_{i}^{l}, s_{j}^{l}}}\left[\left(1-\delta_{s_{i}^{l}, s_{j}^{l}}\right)\left(1+\delta_{s_{i}^{l}, s_{j+1}^{l}}+o\left(\delta_{\max }^{2}\right)\right)\right] \\
& +\frac{o\left(\delta_{\max }^{2}\right)}{K \operatorname{det}(I-\Gamma)}+\frac{o\left(\delta_{\max }^{K}\right)}{K \operatorname{det}(I-\Gamma)^{2}} \\
& =-\frac{1}{K \operatorname{det}(I-\Gamma)}\left[\left(1+\delta_{s_{i}^{l}, s_{j+1}^{l}}\right)\left(e_{s_{i-1}^{l}, s_{j}^{l}}-1\right)\right]+\frac{o\left(\delta_{\max }^{2}\right)}{K \operatorname{det}(I-\Gamma)}+\frac{o\left(\delta_{\max }^{K}\right)}{K \operatorname{det}(I-\Gamma)^{2}} \\
& =-\frac{1}{K\left(1-o\left(\delta_{\max }^{K}\right)\right)}\left[\left(1+\delta_{s_{i}^{l}, s_{j+1}^{l}}\right)\left(e_{s_{i-1}^{l}, s_{j}^{l}}-1\right)\right]+\frac{o\left(\delta_{\max }^{2}\right)}{K\left(1-o\left(\delta_{\max }^{K}\right)\right)}
\end{aligned}
$$




$$
\begin{aligned}
& +\frac{o\left(\delta_{\max }^{K}\right)}{K\left(1-o\left(\delta_{\max }^{K}\right)\right)} \\
= & -\frac{1}{K}\left[\left(1+\delta_{s_{i}^{l}, s_{j+1}^{l}}^{l}\right)\left(e_{s_{i-1}^{l}, s_{j}^{l}}-1\right)\right]+o\left(\delta_{\max }^{2}\right) .
\end{aligned}
$$

\section{Transitions without switch:}

Define the matrix $Q$

$$
Q=\left(\begin{array}{ccccccccc}
0 & \ldots & 0 & \ldots & 0 & \ldots & 0 & \ldots & 0 \\
0 & \ddots & 0 & \ldots & 0 & \ldots & 0 & \ddots & 0 \\
0 & \ldots & q_{j-k_{s_{i}}, j-k_{s_{i}}} & \ldots & 0 & \ldots & 0 & \ldots & 0 \\
0 & \ldots & 0 & \ddots & 0 & \ldots & 0 & \ldots & 0 \\
0 & \ldots & 0 & \ldots & q_{j-m, j-m} & \ldots & 0 & \ldots & 0 \\
0 & \ldots & 0 & \ldots & 0 & \ddots & 0 & \ldots & 0 \\
0 & \ldots & 0 & \ldots & 0 & \ldots & q_{j, j} & \ldots & 0 \\
0 & \ddots & 0 & \ldots & 0 & \ldots & 0 & \ddots & 0 \\
0 & \ldots & 0 & \ldots & 0 & \ldots & 0 & \ldots & 0
\end{array}\right)
$$

where $j=\sum_{h=1}^{i}\left(k_{s_{h}^{l}}+1\right), q_{j-k_{s_{i}^{l}}, j-k_{s_{i}^{l}}}=\operatorname{det}(I-\Gamma), q_{j-m, j-m}=1-\operatorname{det}(I-\Gamma), m=$ $2, \ldots, k_{s_{i}^{l}}-1$, and $q_{j, j}=1$, and the index must follow the above rule.

We can find

$$
\operatorname{det}(I-\Gamma) \frac{\partial \operatorname{sum}(E)}{\partial e_{s_{i}^{l}, s_{i}^{l}}}=\frac{\partial \operatorname{sum}(Q(I-P) E)}{\partial e_{s_{i}^{l}, s_{i}^{l}}}
$$

Considering

$$
\frac{\partial \operatorname{det}(I-\Gamma)}{\partial \delta_{s_{i}^{l}, s_{i}^{l}}}=o\left(\delta_{\max }^{n-1}\right)
$$

the partial derivatives can be obtained

If the batch size $k_{s_{i}^{l}}=2$

$$
\frac{\partial P\left(g^{l}\right)}{\partial \lambda_{s_{i}^{l}, s_{i}^{l}}}
$$




$$
\begin{aligned}
& =\frac{\partial P\left(g^{l}\right)}{\partial \delta_{s_{i}^{l}, s_{i}^{l}}^{l}} \frac{\partial \delta_{s_{i}^{l}, s_{i}^{l}}}{\partial \lambda_{s_{i}^{l}, s_{i}^{l}}}+\frac{\partial P\left(g^{l}\right)}{\partial e_{s_{i}^{l}, s_{i}^{l}}} \frac{\partial e_{s_{i}^{l}, s_{i}^{l}}}{\partial \lambda_{s_{i}^{l}, s_{i}^{l}}} \\
& =-\frac{1}{K \operatorname{det}(I-\Gamma)} \frac{\partial \operatorname{sum}\left(\sum_{i=1}^{K-1} \Gamma^{i}(I-P) E\right)}{\partial \delta_{s_{i}^{l}, s_{i}^{l}}}+\frac{o\left(\delta_{\max }^{K}\right)}{K \operatorname{det}(I-\Gamma)^{2}} \\
& -\frac{e_{s_{i}^{l}, s_{i}^{l}}}{1-\delta_{s_{i}^{l}, s_{i}^{l}}}\left[\frac{1}{K} \frac{\partial \operatorname{sum}(E)}{\partial e_{s_{i}^{l}, s_{i}^{l}}^{l}}+\frac{1}{K \operatorname{det}(I-\Gamma)} \frac{\partial \operatorname{sum}\left(\sum_{i=1}^{K-1} \Gamma^{i}(I-P) E\right)}{\partial e_{s_{i}^{l}, s_{i}^{l}}}\right] \\
& =-\frac{1}{K \operatorname{det}(I-\Gamma)}\left[\frac{\partial \operatorname{sum}\left(\sum_{i=1}^{2} \Gamma^{i}(I-P) E\right)}{\partial \delta_{s_{i}^{l}, s_{i}^{l}}^{l}}+o\left(\delta_{\max }^{2}\right)\right]+\frac{o\left(\delta_{\max }^{K}\right)}{K \operatorname{det}(I-\Gamma)^{2}} \\
& -\frac{1}{K \operatorname{det}(I-\Gamma)} \frac{e_{s_{i}^{l}, s_{i}^{l}}}{1-\delta_{s_{i}^{l}, s_{i}^{l}}}\left[\operatorname{det}(I-\Gamma) \frac{\partial \operatorname{sum}(E)}{\partial e_{s_{i}^{l}, s_{i}^{l}}}+\frac{\partial \operatorname{sum}\left(\sum_{i=1}^{K-1} \Gamma^{i}(I-P) E\right)}{\partial e_{s_{i}^{l}, s_{i}^{l}}}\right] \\
& =-\frac{1}{K \operatorname{det}(I-\Gamma)}\left[\frac{\partial \operatorname{sum}\left(\sum_{i=1}^{2} \Gamma^{i}(I-P) E\right)}{\partial \delta_{s_{i}^{l}, s_{i}^{l}}^{l}}\right]+\frac{o\left(\delta_{\max }^{2}\right)}{K \operatorname{det}(I-\Gamma)}+\frac{o\left(\delta_{\max }^{K}\right)}{K \operatorname{det}(I-\Gamma)^{2}} \\
& -\frac{1}{K \operatorname{det}(I-\Gamma)} \frac{e_{s_{i}^{l}, s_{i}^{l}}}{1-\delta_{s_{i}^{l}, s_{i}^{l}}}\left[\frac{\partial \operatorname{sum}\left(\left(Q+\sum_{i=1}^{K-1} \Gamma^{i}\right)(I-P) E\right)}{\partial e_{s_{i}^{l}, s_{i}^{l}}}\right] \\
& =-\frac{1}{K \operatorname{det}(I-\Gamma)}\left[\left(1+\delta_{s_{i+1}^{l}, s_{i}^{l}}\right)\left(e_{s_{i}^{l}, s_{i-1}^{l}}-e_{s_{i}^{l}, s_{i}}\right)+\delta_{s_{i}^{l}, s_{i-1}^{l}}\left(e_{s_{i-1}^{l}, s_{i-1}^{l}}-e_{s_{i}^{l}, s_{i-1}^{l}}\right)\right] \\
& -\frac{1}{K \operatorname{det}(I-\Gamma)} \frac{e_{s_{i}^{l}, s_{i}^{l}}}{1-\delta_{s_{i}^{l}, s_{i}^{l}}}\left[\left(1-\delta_{s_{i}^{l}, s_{i}^{l}}\right)\left(1+\delta_{s_{i+1}^{l}, s_{i}^{l}}+o\left(\delta_{\text {max }}^{2}\right)\right)\right] \\
& +\frac{o\left(\delta_{\max }^{2}\right)}{K \operatorname{det}(I-\Gamma)}+\frac{o\left(\delta_{\max }^{K}\right)}{K \operatorname{det}(I-\Gamma)^{2}} \\
& =-\frac{1}{K \operatorname{det}(I-\Gamma)}\left[\left(1+\delta_{s_{i+1}^{l}, s_{i}^{l}}\right) e_{s_{i}^{l}, s_{i-1}^{l}}+\delta_{s_{i}^{l}, s_{i-1}^{l}}\left(e_{s_{i-1}^{l}, s_{i-1}^{l}}-e_{s_{i}^{l}, s_{i-1}^{l}}\right)\right] \\
& +\frac{o\left(\delta_{\max }^{2}\right)}{K \operatorname{det}(I-\Gamma)}+\frac{o\left(\delta_{\max }^{K}\right)}{K \operatorname{det}(I-\Gamma)^{2}} \\
& =-\frac{1}{K\left(1-o\left(\delta_{\max }^{K}\right)\right)}\left[\left(1+\delta_{s_{i+1}^{l}, s_{i}^{l}}\right) e_{s_{i}^{l}, s_{i-1}^{l}}+\delta_{s_{i}^{l}, s_{i-1}^{l}}\left(e_{s_{i-1}^{l}, s_{i-1}^{l}}-e_{s_{i}^{l}, s_{i-1}^{l}}\right)\right] \\
& +\frac{o\left(\delta_{\max }^{2}\right)}{K\left(1-o\left(\delta_{\max }^{K}\right)\right)}+\frac{o\left(\delta_{\max }^{K}\right)}{K\left(1-o\left(\delta_{\max }^{K}\right)\right)} \\
& =-\frac{1}{K}\left[\left(1+\delta_{s_{i+1}^{l}, s_{i}^{l}}\right) e_{s_{i}^{l}, s_{i-1}^{l}}+\delta_{s_{i}^{l}, s_{i-1}^{l}}\left(e_{s_{i-1}^{l}, s_{i-1}^{l}}-e_{s_{i}^{l}, s_{i-1}^{l}}\right)\right]+o\left(\delta_{\text {max }}^{2}\right) \text {. } \\
& \frac{\partial P\left(g^{l}\right)}{\partial \mu_{s_{i}^{l}, s_{i}^{l}}} \\
& =\frac{\partial P\left(g^{l}\right)}{\partial \delta_{s_{i}^{l}, s_{i}^{l}}} \frac{\partial \delta_{s_{i}^{l}, s_{i}^{l}}}{\partial \mu_{s_{i}^{l}, s_{i}^{l}}}+\frac{\partial P\left(g^{l}\right)}{\partial e_{s_{i}^{l}, s_{i}^{l}}^{l}} \frac{\partial e_{s_{i}^{l}, s_{i}^{l}}}{\partial \mu_{s_{i}^{l}, s_{i}^{l}}}
\end{aligned}
$$




$$
\begin{aligned}
& =-\frac{1}{K \operatorname{det}(I-\Gamma)} \frac{\partial \operatorname{sum}\left(\sum_{i=1}^{K-1} \Gamma^{i}(I-P) E\right)}{\partial \delta_{s_{i}^{l}, s_{i}^{l}}}+\frac{o\left(\delta_{\max }^{K}\right)}{K \operatorname{det}(I-\Gamma)^{2}} \\
& +\frac{1-e_{s_{i}^{l}, s_{i}^{l}}}{1-\delta_{s_{i}^{l}, s_{i}^{l}}}\left[\frac{1}{K} \frac{\partial \operatorname{sum}(E)}{\partial e_{s_{i}^{l}, s_{i}^{l}}}+\frac{1}{K \operatorname{det}(I-\Gamma)} \frac{\partial \operatorname{sum}\left(\sum_{i=1}^{K-1} \Gamma^{i}(I-P) E\right)}{\partial e_{s_{i}^{l}, s_{i}^{l}}}\right] \\
& =-\frac{1}{K \operatorname{det}(I-\Gamma)}\left[\frac{\partial \operatorname{sum}\left(\sum_{i=1}^{2} \Gamma^{i}(I-P) E\right)}{\partial \delta_{s_{i}^{l}, s_{i}^{l}}^{l}}+o\left(\delta_{\max }^{2}\right)\right]+\frac{o\left(\delta_{\max }^{K}\right)}{K \operatorname{det}(I-\Gamma)^{2}} \\
& +\frac{1}{K \operatorname{det}(I-\Gamma)} \frac{1-e_{s_{i}^{l}, s_{i}^{l}}}{1-\delta_{s_{i}^{l}, s_{i}^{l}}}\left[\operatorname{det}(I-\Gamma) \frac{\partial \operatorname{sum}(E)}{\partial e_{s_{i}^{l}, s_{i}^{l}}}+\frac{\partial \operatorname{sum}\left(\sum_{i=1}^{K-1} \Gamma^{i}(I-P) E\right)}{\partial e_{s_{i}^{l}, s_{i}^{l}}}\right] \\
& =-\frac{1}{K \operatorname{det}(I-\Gamma)}\left[\frac{\partial \operatorname{sum}\left(\sum_{i=1}^{2} \Gamma^{i}(I-P) E\right)}{\partial \delta_{s_{i}^{l}, s_{i}^{l}}}\right]+\frac{o\left(\delta_{\max }^{2}\right)}{K \operatorname{det}(I-\Gamma)}+\frac{o\left(\delta_{\max }^{K}\right)}{K \operatorname{det}(I-\Gamma)^{2}} \\
& +\frac{1}{K \operatorname{det}(I-\Gamma)} \frac{1-e_{s_{i}^{l}, s_{i}^{l}}}{1-\delta_{s_{i}^{l}, s_{i}^{l}}}\left[\frac{\partial \operatorname{sum}\left(\left(Q+\sum_{i=1}^{K-1} \Gamma^{i}\right)(I-P) E\right)}{\partial e_{s_{i}^{l}, s_{i}^{l}}}\right] \\
& =-\frac{1}{K \operatorname{det}(I-\Gamma)}\left[\left(1+\delta_{s_{i+1}^{l}, s_{i}^{l}}\right)\left(e_{s_{i}^{l}, s_{i-1}^{l}}-e_{s_{i}^{l}, s_{i}}\right)+\delta_{s_{i}^{l}, s_{i-1}^{l}}\left(e_{s_{i-1}^{l}, s_{i-1}^{l}}-e_{s_{i}^{l}, s_{i-1}^{l}}\right)\right] \\
& +\frac{1}{K \operatorname{det}(I-\Gamma)} \frac{1-e_{s_{i}^{l}, s_{i}^{l}}}{1-\delta_{s_{i}^{l}, s_{i}^{l}}^{l}}\left[\left(1-\delta_{s_{i}^{l}, s_{i}^{l}}\right)\left(1+\delta_{s_{i+1}^{l}, s_{i}^{l}}+o\left(\delta_{\max }^{2}\right)\right)\right] \\
& +\frac{o\left(\delta_{\max }^{2}\right)}{K \operatorname{det}(I-\Gamma)}+\frac{o\left(\delta_{\max }^{K}\right)}{K \operatorname{det}(I-\Gamma)^{2}} \\
& =-\frac{1}{K \operatorname{det}(I-\Gamma)}\left[\left(1+\delta_{s_{i+1}^{l}, s_{i}^{l}}\right)\left(e_{s_{i}^{l}, s_{i-1}^{l}}-1\right)+\delta_{s_{i}^{l}, s_{i-1}^{l}}\left(e_{s_{i-1}^{l}, s_{i-1}^{l}}-e_{s_{i}^{l}, s_{i-1}^{l}}\right)\right] \\
& +\frac{o\left(\delta_{\max }^{2}\right)}{K \operatorname{det}(I-\Gamma)}+\frac{o\left(\delta_{\max }^{K}\right)}{K \operatorname{det}(I-\Gamma)^{2}} \\
& =-\frac{1}{K\left(1-o\left(\delta_{\max }^{K}\right)\right)}\left[\left(1+\delta_{s_{i+1}^{l}, s_{i}^{l}}\right)\left(e_{s_{i}^{l}, s_{i-1}^{l}}-1\right)+\delta_{s_{i}^{l}, s_{i-1}^{l}}\left(e_{s_{i-1}^{l}, s_{i-1}^{l}}-e_{s_{i}^{l}, s_{i-1}^{l}}\right)\right] \\
& +\frac{o\left(\delta_{\max }^{2}\right)}{K\left(1-o\left(\delta_{\max }^{K}\right)\right)}+\frac{o\left(\delta_{\max }^{K}\right)}{K\left(1-o\left(\delta_{\max }^{K}\right)\right)} \\
& =-\frac{1}{K}\left[\left(1+\delta_{s_{i+1}^{l}, s_{i}^{l}}\right)\left(e_{s_{i}^{l}, s_{i-1}^{l}}-1\right)+\delta_{s_{i}^{l}, s_{i-1}^{l}}\left(e_{s_{i-1}^{l}, s_{i-1}^{l}}-e_{s_{i}^{l}, s_{i-1}^{l}}\right)\right]+o\left(\delta_{\text {max }}^{2}\right) \text {. }
\end{aligned}
$$

If the batch size $k_{s_{i}^{l}}>2$

$$
\begin{aligned}
& \frac{\partial P\left(g^{l}\right)}{\partial \lambda_{s_{i}^{l}, s_{i}^{l}}} \\
= & \frac{\partial P\left(g^{l}\right)}{\partial \delta_{s_{i}^{l}, s_{i}^{l}}^{l}} \frac{\partial \delta_{s_{i}^{l}, s_{i}^{l}}}{\partial \lambda_{s_{i}^{l}, s_{i}^{l}}^{l}}+\frac{\partial P\left(g^{l}\right)}{\partial e_{s_{i}^{l}, s_{i}^{l}}} \frac{\partial e_{s_{i}^{l}, s_{i}^{l}}}{\partial \lambda_{s_{i}^{l}, s_{i}^{l}}}
\end{aligned}
$$




$$
\begin{aligned}
& =-\frac{1}{K \operatorname{det}(I-\Gamma)} \frac{\partial \operatorname{sum}\left(\sum_{i=1}^{K-1} \Gamma^{i}(I-P) E\right)}{\partial \delta_{s_{i}^{l}, s_{i}^{l}}}+\frac{o\left(\delta_{\max }^{K}\right)}{K \operatorname{det}(I-\Gamma)^{2}} \\
& -\frac{e_{s_{i}^{l}, s_{i}^{l}}}{1-\delta_{s_{i}^{l}, s_{i}^{l}}^{l}}\left[\frac{1}{K} \frac{\partial \operatorname{sum}(E)}{\partial e_{s_{i}^{l}, s_{i}^{l}}}+\frac{1}{K \operatorname{det}(I-\Gamma)} \frac{\partial \operatorname{sum}\left(\sum_{i=1}^{K-1} \Gamma^{i}(I-P) E\right)}{\partial e_{s_{i}^{l}, s_{i}^{l}}}\right] \\
& =-\frac{1}{K \operatorname{det}(I-\Gamma)}\left[\frac{\partial \operatorname{sum}\left(\sum_{i=1}^{2} \Gamma^{i}(I-P) E\right)}{\partial \delta_{s_{i}^{l}, s_{i}^{l}}}+o\left(\delta_{\max }^{2}\right)\right]+\frac{o\left(\delta_{\max }^{K}\right)}{K \operatorname{det}(I-\Gamma)^{2}} \\
& -\frac{1}{K \operatorname{det}(I-\Gamma)} \frac{e_{s_{i}^{l}, s_{i}^{l}}}{1-\delta_{s_{i}^{l}, s_{i}^{l}}}\left[\operatorname{det}(I-\Gamma) \frac{\partial \operatorname{sum}(E)}{\partial e_{s_{i}^{l}, s_{i}^{l}}}+\frac{\partial \operatorname{sum}\left(\sum_{i=1}^{K-1} \Gamma^{i}(I-P) E\right)}{\partial e_{s_{i}^{l}, s_{i}^{l}}}\right] \\
& =-\frac{1}{K \operatorname{det}(I-\Gamma)}\left[\frac{\partial \operatorname{sum}\left(\sum_{i=1}^{2} \Gamma^{i}(I-P) E\right)}{\partial \delta_{s_{i}^{l}, s_{i}^{l}}}\right]+\frac{o\left(\delta_{\max }^{2}\right)}{K \operatorname{det}(I-\Gamma)}+\frac{o\left(\delta_{\max }^{K}\right)}{K \operatorname{det}(I-\Gamma)^{2}} \\
& -\frac{1}{K \operatorname{det}(I-\Gamma)} \frac{e_{s_{i}^{l}, s_{i}^{l}}}{1-\delta_{s_{i}^{l}, s_{i}^{l}}}\left[\frac{\partial \operatorname{sum}\left(\left(Q+\sum_{i=1}^{K-1} \Gamma^{i}\right)(I-P) E\right)}{\partial e_{s_{i}^{l}, s_{i}^{l}}}\right] \\
& =-\frac{1}{K \operatorname{det}(I-\Gamma)}\left[\left(1+\delta_{s_{i}^{l}, s_{i}^{l}}\right)\left(e_{s_{i}^{l}, s_{i-1}^{l}}-e_{s_{i}^{l}, s_{i}^{l}}\right)+\delta_{s_{i}^{l}, s_{i-1}^{l}}\left(e_{s_{i-1}^{l}, s_{i-1}^{l}}-e_{s_{i}^{l}, s_{i-1}^{l}}\right)\right. \\
& \left.+\delta_{s_{i}^{l}, s_{i}^{l}}\left(e_{s_{i}^{l}, s_{i-1}^{l}}-e_{s_{i}^{l}, s_{i}^{l}}\right)\right]-\frac{1}{K \operatorname{det}(I-\Gamma)} \frac{e_{s_{i}^{l}, s_{i}^{l}}}{1-\delta_{s_{i}^{l}, s_{i}^{l}}} \\
& \cdot\left[\left(1-\delta_{s_{i}^{l}, s_{i}^{l}}\right)\left(1+\delta_{s_{i+1}^{l}, s_{i}^{l}}+\left(k_{s_{i}^{l}}-2\right)\left(1+\delta_{s_{i}^{l}, s_{i}^{l}}\right)+o\left(\delta_{\max }^{2}\right)\right)\right] \\
& +\frac{o\left(\delta_{\max }^{2}\right)}{K \operatorname{det}(I-\Gamma)}+\frac{o\left(\delta_{\max }^{K}\right)}{K \operatorname{det}(I-\Gamma)^{2}} \\
& =-\frac{1}{K\left(1-o\left(\delta_{\text {max }}^{K}\right)\right)}\left[\left(1+\delta_{s_{i}^{l}, s_{i}^{l}}\right) e_{s_{i}^{l}, s_{i-1}^{l}}+\left(k_{s_{i}^{l}}-3\right)\left(1+\delta_{s_{i}^{l}, s_{i}^{l}}\right) e_{s_{i}^{l}, s_{i}^{l}}\right. \\
& \left.+\left(1+\delta_{s_{i+1}^{l}, s_{i}^{l}}\right) e_{s_{i}^{l}, s_{i}^{l}}\right] \\
& -\frac{1}{K\left(1-o\left(\delta_{\max }^{K}\right)\right)}\left[\delta_{s_{i}^{l}, s_{i-1}^{l}}\left(e_{s_{i-1}^{l}, s_{i-1}^{l}}-e_{s_{i}^{l}, s_{i-1}^{l}}\right)+\delta_{s_{i}^{l}, s_{i}^{l}}\left(e_{s_{i}^{l}, s_{i-1}^{l}}-e_{s_{i}^{l}, s_{i}^{l}}\right)\right] \\
& +\frac{o\left(\delta_{\max }^{2}\right)}{K\left(1-o\left(\delta_{\max }^{K}\right)\right)}+\frac{o\left(\delta_{\max }^{K}\right)}{K\left(1-o\left(\delta_{\max }^{K}\right)\right)} \\
& =-\frac{1}{K}\left[\left(1+\delta_{s_{i}^{l}, s_{i}^{l}}\right) e_{s_{i}^{l}, s_{i-1}^{l}}+\left(k_{s_{i}^{l}}-3\right)\left(1+\delta_{s_{i}^{l}, s_{i}^{l}}\right) e_{s_{i}^{l}, s_{i}^{l}}+\left(1+\delta_{s_{i+1}^{l}, s_{i}^{l}}\right) e_{s_{i}^{l}, s_{i}^{l}}\right] \\
& -\frac{1}{K}\left[\delta_{s_{i}^{l}, s_{i-1}^{l}}\left(e_{s_{i-1}^{l}, s_{i-1}^{l}}^{l}-e_{s_{i}^{l}, s_{i-1}^{l}}\right)+\delta_{s_{i}^{l}, s_{i}^{l}}\left(e_{s_{i}^{l}, s_{i-1}^{l}}-e_{s_{i}^{l}, s_{i}^{l}}\right)\right]+o\left(\delta_{\max }^{2}\right) \text {. } \\
& \frac{\partial P\left(g^{l}\right)}{\partial \mu_{s_{i}^{l}, s_{i}^{l}}}
\end{aligned}
$$




$$
\begin{aligned}
& =\frac{\partial P\left(g^{l}\right)}{\partial \delta_{s_{i}^{l}, s_{i}^{l}}} \frac{\partial \delta_{s_{i}^{l}, s_{i}^{l}}}{\partial \mu_{s_{i}^{l}, s_{i}^{l}}}+\frac{\partial P\left(g^{l}\right)}{\partial e_{s_{i}^{l}, s_{i}^{l}}} \frac{\partial e_{s_{i}^{l}, s_{i}^{l}}}{\partial \mu_{s_{i}^{l}, s_{i}^{l}}} \\
& =-\frac{1}{K \operatorname{det}(I-\Gamma)} \frac{\partial \operatorname{sum}\left(\sum_{i=1}^{K-1} \Gamma^{i}(I-P) E\right)}{\partial \delta_{s_{i}^{l}, s_{i}^{l}}}+\frac{o\left(\delta_{\max }^{K}\right)}{K \operatorname{det}(I-\Gamma)^{2}} \\
& +\frac{1-e_{s_{i}^{l}, s_{i}^{l}}}{1-\delta_{s_{i}^{l}, s_{i}^{l}}}\left[\frac{1}{K} \frac{\partial \operatorname{sum}(E)}{\partial e_{s_{i}^{l}, s_{i}^{l}}}+\frac{1}{K \operatorname{det}(I-\Gamma)} \frac{\partial \operatorname{sum}\left(\sum_{i=1}^{K-1} \Gamma^{i}(I-P) E\right)}{\partial e_{s_{i}^{l}, s_{i}^{l}}}\right] \\
& =-\frac{1}{K \operatorname{det}(I-\Gamma)}\left[\frac{\partial \operatorname{sum}\left(\sum_{i=1}^{2} \Gamma^{i}(I-P) E\right)}{\partial \delta_{s_{i}^{l}, s_{i}^{l}}}+o\left(\delta_{\max }^{2}\right)\right]+\frac{o\left(\delta_{\max }^{K}\right)}{K \operatorname{det}(I-\Gamma)^{2}} \\
& +\frac{1}{K \operatorname{det}(I-\Gamma)} \frac{1-e_{s_{i}^{l}, s_{i}^{l}}}{1-\delta_{s_{i}^{l}, s_{i}^{l}}}\left[\operatorname{det}(I-\Gamma) \frac{\partial \operatorname{sum}(E)}{\partial e_{s_{i}^{l}, s_{i}^{l}}}+\frac{\partial \operatorname{sum}\left(\sum_{i=1}^{K-1} \Gamma^{i}(I-P) E\right)}{\partial e_{s_{i}^{l}, s_{i}^{l}}}\right] \\
& =-\frac{1}{K \operatorname{det}(I-\Gamma)}\left[\frac{\partial \operatorname{sum}\left(\sum_{i=1}^{2} \Gamma^{i}(I-P) E\right)}{\partial \delta_{s_{i}^{l}, s_{i}^{l}}}\right]+\frac{o\left(\delta_{\max }^{2}\right)}{K \operatorname{det}(I-\Gamma)}+\frac{o\left(\delta_{\max }^{K}\right)}{K \operatorname{det}(I-\Gamma)^{2}} \\
& +\frac{1}{K \operatorname{det}(I-\Gamma)} \frac{1-e_{s_{i}^{l}, s_{i}^{l}}}{1-\delta_{s_{i}^{l}, s_{i}^{l}}}\left[\frac{\partial \operatorname{sum}\left(\left(Q+\sum_{i=1}^{K-1} \Gamma^{i}\right)(I-P) E\right)}{\partial e_{s_{i}^{l}, s_{i}^{l}}}\right] \\
& =-\frac{1}{K \operatorname{det}(I-\Gamma)}\left[\left(1+\delta_{s_{i}^{l}, s_{i}^{l}}\right)\left(e_{s_{i}^{l}, s_{i-1}^{l}}-e_{s_{i}^{l}, s_{i}^{l}}\right)+\delta_{s_{i}^{l}, s_{i-1}^{l}}\left(e_{s_{i-1}^{l}, s_{i-1}^{l}}-e_{s_{i}^{l}, s_{i-1}^{l}}\right)\right. \\
& \left.+\delta_{s_{i}^{l}, s_{i}^{l}}\left(e_{s_{i}^{l}, s_{i-1}^{l}}-e_{s_{i}^{l}, s_{i}^{l}}\right)\right]+\frac{1}{K \operatorname{det}(I-\Gamma)} \frac{1-e_{s_{i}^{l}, s_{i}^{l}}}{1-\delta_{s_{i}^{l}, s_{i}^{l}}} \\
& \cdot\left[\left(1-\delta_{s_{i}^{l}, s_{i}^{l}}\right)\left(1+\delta_{s_{i+1}^{l}, s_{i}^{l}}+\left(k_{s_{i}^{l}}-2\right)\left(1+\delta_{s_{i}^{l}, s_{i}^{l}}\right)+o\left(\delta_{\max }^{2}\right)\right)\right] \\
& +\frac{o\left(\delta_{\max }^{2}\right)}{K \operatorname{det}(I-\Gamma)}+\frac{o\left(\delta_{\max }^{K}\right)}{K \operatorname{det}(I-\Gamma)^{2}} \\
& =-\frac{1}{K\left(1-o\left(\delta_{\max }^{K}\right)\right)}\left[\left(1+\delta_{s_{i}^{l}, s_{i}^{l}}\right)\left(e_{s_{i}^{l}, s_{i-1}^{l}}-1\right)+\left(k_{s_{i}^{l}}-3\right)\left(1+\delta_{s_{i}^{l}, s_{i}^{l}}\right)\left(e_{s_{i}^{l}, s_{i}^{l}}-1\right)\right. \\
& \left.+\left(1+\delta_{s_{i+1}^{l}, s_{i}^{l}}\right)\left(e_{s_{i}^{l}, s_{i}^{l}}-1\right)\right] \\
& -\frac{1}{K\left(1-o\left(\delta_{\max }^{K}\right)\right)}\left[\delta_{s_{i}^{l}, s_{i-1}^{l}}\left(e_{s_{i-1}^{l}, s_{i-1}^{l}}-e_{s_{i}^{l}, s_{i-1}^{l}}\right)+\delta_{s_{i}^{l}, s_{i}^{l}}\left(e_{s_{i}^{l}, s_{i-1}^{l}}-e_{s_{i}^{l}, s_{i}^{l}}\right)\right] \\
& +\frac{o\left(\delta_{\max }^{2}\right)}{K\left(1-o\left(\delta_{\max }^{K}\right)\right)}+\frac{o\left(\delta_{\max }^{K}\right)}{K\left(1-o\left(\delta_{\max }^{K}\right)\right)} \\
& =-\frac{1}{K}\left[\left(1+\delta_{s_{i}^{l}, s_{i}^{l}}\right)\left(e_{s_{i}^{l}, s_{i-1}^{l}}-1\right)+\left(k_{s_{i}^{l}}-3\right)\left(1+\delta_{s_{i}^{l}, s_{i}^{l}}\right)\left(e_{s_{i}^{l}, s_{i}^{l}}-1\right)\right. \\
& \left.+\left(1+\delta_{s_{i+1}^{l}, s_{i}^{l}}\right)\left(e_{s_{i}^{l}, s_{i}^{l}}-1\right)\right] \\
& -\frac{1}{K}\left[\delta_{s_{i}^{l}, s_{i-1}^{l}}\left(e_{s_{i-1}^{l}, s_{i-1}^{l}}-e_{s_{i}^{l}, s_{i-1}^{l}}\right)+\delta_{s_{i}^{l}, s_{i}^{l}}\left(e_{s_{i}^{l}, s_{i-1}^{l}}-e_{s_{i}^{l}, s_{i}^{l}}\right)\right]+o\left(\delta_{\max }^{2}\right) .
\end{aligned}
$$




\section{REFERENCES}

[1] N. Viswanadham and Y. Narahari, Performance Modeling of Automated Manufacturing System, Prentice Hall, 1992.

[2] J.A. Buzacott and J.G. Shantikumar, Stochastic Models of Manufacturing Systems, Prentice Hall, 1993.

[3] H. Tempelmeier and H. Kuhn, Flexible Manufacturing Systems: Decision Support for Design and Operation, Wiley-Interscience, 1993.

[4] M. Zhou and K. Venkatesh, Modeling, Simulation and Control of Flexible Manufacturing Systems: A Petri Net Approach, World Scientific Publishing Company, 1999.

[5] J.A. Buzacott and D.D. Yao, "Flexible Manufacturing Systems: A Review of Analytical Models," Management Science, vol. 32, pp. 890-905, 1986.

[6] Y.P. Gupta and S. Goyal, "Flexibility of Manufacturing Systems: Concepts and Measurements," European Journal of Operations Research, vol. 43, pp. 119-135, 1989 .

[7] A.K. Sethi and P.S. Sethi, "Flexibility in Manufacturing: A Survey," International Journal of Flexible Manufacturing Systems, vol. 2, pp. 289-328, 1990.

[8] M. Barad and S.Y. Nof, "CIM Flexibility Measures: A Review and a Framework for Analysis and Applicability Assessment," International Journal of Computer Integrated manufacturing, vol. 10, pp. 296-308, 1997.

[9] A. De Toni and S. Tonchia, "Manufacturing Flexibility: A Literature Review," International Journal of Production Research, vol. 36, pp. 1587-1617, 1998.

[10] D.E. D'souza and F.P. Williams, "Toward a Taxonomy of Manufacturing Flexibility Dimensions," Journal of Operations Management, vol. 18, pp. 577-593, 2000.

[11] R. Beach, A.P. Juhlemann, D.H.R. Price, A. Paterson and J.A. Sharp, "A Review of Manufacturing Flexibility," European Journal of Operational Research, vol. 122, pp. 41-57, 2000.

[12] J. Bengtsson, "Manufacturing Flexibility and Real Options: A Review," International Journal of Production Economics, vol. 74, pp. 213-224, 2001.

[13] D. Shi and R.L. Daniels, "A Survey of Manufacturing Flexibility: Implications for e-Business Flexibility," IBM Systems Journal, vol. 42, pp. 414-427, 2003. 
[14] J. Payne and V. Cariapa, "A Fixture Repeatability and Reproducibility Measure to Predict the Quality of Machined Parts," International Journal of Production Research, vol. 38, pp. 4763-4781, 2000.

[15] R.R. Inman, D.E. Blumenfeld, N. Huang and J. Li, "Production System Design for Quality: Research Opportunities from Automotive Industry Prospective," International Journal of Production Research, vol. 41, pp. 1953-1971, 2003.

[16] J.E. Brady and T.T. Allen, "Six Sigma Literature: A Review and Agenda for Future Research," Quality and Reliability Engineering International, vol. 22, pp. 335-367, 2006.

[17] F. Tsung, Y. Li, and M. Jin, "Statistical Process Control for Multistage Manufacturing and Service Operation: A Review and Some Extensions," International Journal of Services Operations and Informatics, vol. 3, pp. 191-204, 2008.

[18] T. Lager, "The Industrial Usability of Euality Function Deployment: A Literature Review and Synthesis on a Meta-level," RED Management, vol. 35, pp. 409-426, 2005.

[19] J.R. Sharma, A.M. Rawani, and M. Barahate, "Quality Function Deployment: A Comprehensive Literature Review," International Journal of Data Analysis Techniques and Strategies, vol. 1, pp. 78-103, 2008.

[20] J. Shi and S. Zhou, "Quality Control and Improvement for Multistage Systems: A Survey," IIE Transactions, vol. 41, pp. 744-753, 2009.

[21] J. Li and N. Huang, "Quality Evaluation in Flexible Manufacturing Systems: A Markovian Approach," Mathematical Problems in Engineering, vol. 2007, article ID 57128, 2007.

[22] J. Li, D.E. Blumenfeld and S.P. Marin, "Production System Design for Quality Robustness," IIE Transactions, vol. 40, pp. 162-176, 2008.

[23] D.E. Zoia, "Harbour Outlines Who's Winning and Why," WardsAuto.com, September 27, 2005.

[24] A. Bolat and C. Yano, "Procedures to Analyze the Tradeoffs between Costs of Setup and Utility Work for Automobile Assembly Lines," Report 89-3, Department of IOE, University of Michigan, Ann Arbor, MI, 1989.

[25] P. Van Hentenryck, Y. Deville and C. Yeng, " A Generic Arc-consistency Algorithm and Its Specializations," Artificial Intelligence, vol. 57, pp. 291-321, 1992.

[26] P. Van Hentenryck, The OPL Optimization Programming Language, MIT Press, 1999.

[27] C. Gagne, M. Gravel, and W. L. Price, "Solving real car sequencing problems with ant colony optimization," European journal of operational research, vol. 174, pp. 1427-1448, 2006. 
[28] C. Solnon, "Solving Car Sequencing Problems with Artificial Ants," European Conference on Artificial Intelligence (ECAI-2000),pp.118-122, Berlin, Germany, 2000.

[29] J. Gottlieb, M. Puchta and C. Solnon, "A Study of Greedy, Local Search and Ant Colony Optimization Approaches for Car Sequencing Problems," Applications of Evolutionary Computing, Lecture Notes in Computer Science, Springer-Verlag, Heidelberg, pp. 246-257, 2003.

[30] M. Prandtstetter and G. R. Raidl, "An Integer Linear Programming Approach and A Hybrid Variable Neighborhood Search for the Car Sequencing Problem," European Journal of Operational Research, vol. 191, pp. 1004-1022, 2008.

[31] J. Li and S.M. Meerkov, Production Systems Engineering, Springer, New York, 2009

[32] C.-T. Kuo, J.T. Lim and S.M. Meerkov, "Bottlenecks in Serial Production Lines: A System-Theorectic Approach," Mathematical Problems in Engineering, vol. 2, pp. 233-276, 1996.

[33] S.-Y. Chiang, C.-T. Kuo and S.M. Meerkov, "Bottlenecks in Markovian Production Lines: A Systems Approach," IEEE Transaction on Robotics and Automation, vol. 14, pp. 352-359, 1998.

[34] S.-Y. Chiang, C.-T. Kuo, J.-T. Lim and S.M. Meerkov, "Improvability in Assembly Systems II: Improvability Indicators and Case Study," Mathematical Problems in Engineering, vol. 6, pp. 359-393, 2000.

[35] S.-Y. Chiang, C.-T. Kuo and S.M. Meerkov, "c-Bottlenecks in Serial Production Lines: Identification and Application," Mathematical Problems in Engineering, vol. 7, pp. 543-578, 2001.

[36] S.N. Ching, S.M. Meerkov and L. Zhang, "Assembly Systems with Nonexponential Machines: Throughput and Bottlenecks," Nonlinear AnalysisTheory and Applications, vol. 69, pp. 911-917, 2008.

[37] S. Biller, J. Li, S.P. Marin, S.M. Meerkov and L. Zhang, "Bottlenecks in Production Lines with Rework: A Systems Approach," IEEE Transactions on Automation Science and Engineering, DOI:10.1109/TASE.2009.2023463, 2010.

[38] J. Li and S.M. Meerkov, "Bottlenecks with Respect to Due-Time Performance in Pull Serial Production Lines," Mathematical Problems in Engineering, vol. 5, pp. $479-498,2000$.

[39] C. Galitsky and E. Worrell, "Energy Efficiency Improvement and Cost Saving Opportunities for the Vehicle Assembly Industry," Lawrence Berkeley National Laboratory (LBNL-50939-Revision), 2008. 
[40] T. Kolta, "Selecting Equipment to Control Air Pollution from Automotive Painting Operations," Society of Automotive Engineers (SAE) International Congress and Exposition, 1992.

[41] F.F. Chen and E.E. Adams, "The Impact of Flexible Manufacturing Systems on Productivity and Quality," IEEE Transactions of Engineering Management, vol. 38, pp. 33-45, 1991.

[42] P. Crosby, Quality is Free, New York: New American Library, 1979.

[43] G. Da Silverira, D. Borenstein and F.S. Fogliatto, "Mass Customization: Literature Review and Research Directions," International Journal of Production Economics, vol. 72, pp. 1-13, 2001.

[44] D.C. Montgomery, Introduction to Statistical Quality Control, 3rd Edition, Wiley, New York, 1996.

[45] S. Shingo, Zero Quality Control: Source Inspection and the Poka-Yoke System, Productivity Press, Cambridge, 1986.

[46] Y.K. Son and C.S. Park, "Economic Measure of Productivity, Quality and Flexibility in Advanced Manufacturing Systems," Journal of Manufacturing Systems, vol. 6, pp. 193-207, 1987.

[47] N. Von Hop and K. Ruengsak, "Fuzzy Estimation for Manufacturing Flexibility," International Journal of Production Research, vol. 43, pp. 3605-3617, 2005.

[48] T.C. Powell, "Total Quality Management as Competitive Advantage: A Review and Empirical Study," Strategic Management Journal, vol. 16, No. 1, pp. 15-37, 1995.

[49] S.L. Ahire, R. Landeros and D.Y. Golhar, "Total Quality Management: A Literature Review and an Agenda for Future Research," Production and Operations Management, vol. 4, No. 3, pp. 277-306, 1995.

[50] D.I. Prajogo and A.S. Sohal, "TQM and Innovation: A Literature Review and Research Framework," Technovation, vol. 21, pp. 539-558, 2001.

[51] R. Sousa and C.A. Voss, "Quality Management Re-visited: A Reflective Review and Agenda for Future Research," Journal of Operations Management, vol. 20, pp. 91-109, 2002.

[52] L.-K. Chan and M.-L. Wu, "Quality Function Deployment: A Literature Review," European Journal of Operational Research, vol. 143, pp. 463-497, 2002.

[53] S.R. Devadasan, N. Kathiravan and V. Thirunavukkarasu, "Theory and Practice of Total Quality Function Deployment," The TQM Magazine, vol. 18, No. 2, pp. 143-161, 2006. 
[54] E.B. Martin, A.J. Morris and C. Kiparissides, "Manufacturing Performance Enhancement Through Multivariate Statistical Process Control," Annual Reviews in Control, vol. 23, pp. 35-44, 1999.

[55] B. Mason and J. Antony, "Statistical Process Control: An Essential Ingredient for Improving Service and Manufacturing Quality," Managing Service Quality, vol. 10, No. 4, pp. 233-238, 2000.

[56] J.S. Oakland, Statistical Process Control, Woburn, MA: ButterworthHeinemann, 1996.

[57] F. Tsung, Y. Li and M. Jin, "Statistical Process Control for Multistage Manufacturing and Service Operations: A Review and Some Extensions," International Journal of Services Operations and Informatics, vol. 3, No. 2, pp. 191-204, 2008.

[58] S.R. Das and C. Canel, "An Algorithm for Scheduling Batches of Parts in a Mulit-cell Flexible Manufacturing System," International Journal of Production Economics, vol. 97, pp. 247-262, 2005.

[59] D. Quadt and H. Kuhn, "Batch Scheduling of Jobs with Identical Process Times on Flexible Flow Lines," International Journal of Production Economics, vol. 105, pp. 385-401, 2007.

[60] B.D. Parello, W.C. Kabat and L. Wos, "Job-shop Scheduling Using Automated Reasoning: A Case Study of the Car Sequencing Problem," Journal of Automated Reasoning, vol. 2, pp. 1-42, 1986.

[61] A. Drexl and A. Kimms, "Sequencing JIT Mixed-model Assembly Lines under Station-load and Part-usage Constraints," Management Science, vol. 47, pp. 480491, 2001.

[62] T. Kis, "On the complexity of the car sequencing problem," Operations Research Letters, Vol. 32, pp. 331-335, 2004.

[63] S. Spieckermann, K. Gutenschwager and S. Vo $\beta$, "A Sequential Ordering Problem in Automotive Paint Shops," International Journal of Production Research, vol. 42, No. 9, pp. 1865-1878, 2004.

[64] M. Lahmar, H. Ergan, and S. Benjaafar, "Resequencing and Feature Assignment on an Automated Assembly Line," IEEE Transactions on Robotics and Automation, vol. 19, pp. 89-102, 2003.

[65] M. Lahmar and S. Benjaafar, "Sequencing with Limited Flexibility," IIE Transactions, vol. 39, pp. 937-955, 2007.

[66] J.M. Swaminathan and T. R. Nitsch, "Managing Product Variety in Automobile Assembly: The Importance of the Sequencing Point," Interfaces, vol. 37, pp. 324-333, 2007. 
[67] S. Takahashi, K. Toda, K. Ichihara and K. Uchiyama, "Recent Approaches for Saving Energy in Automotive Painting," International Body Engineering Conference and Exposition, Detroit, Michigan, 1999.

[68] R. Nallicheri, Automotive Painting: An Economic and Strategic Analysis, M.S. thesis, Sloan School of Management, Massachusetts Institute of Technology, Cambridge, MA, 1993.

[69] J. Geoffrey, A. Kemppainen and D. Shonnard, "Assessment of the Automobile Assembly Paint Process for Energy, Environmental, and Ecomonic Improvement," Journal of Industrial Ecology, vol. 8, pp. 173-191, 2004.

[70] S. Papasavva, S. Kia, J. Claya and R. Gunther, "Characterization of Automotive Paints: An Environmental Impact Analysis," Progress in Organic Coatings, vol. 43, pp. 193-206, 2001.

[71] G.J. Roelant, A.J. Kemppainen and D.R. Shonnard, "Assessment of the Automobile Assembly Paint Process for Energy, Environmental, and Economic Improvement," Journal of Industrial Econology, vol. 8, pp. 173-191, 2004.

[72] N. Chardoul, C. Davis, S. Grasman and C. West, "Paint Spray Booth Cleaning Practices in the Automotive Industry," 1995 National Pollution Prevention Center Case Sutdy, American Automobile Manufacturers Association and National Pollution Prevention Center, 1995.

[73] US Environmental Protection Agency, "Automotive Assembly Plant Spray Booth Cleaning Emission Reduction Technology Review," EPA-453/E-94-029, Control Technology Center, 1994.

[74] J.-T. Lim, S.M. Meerkov and F. Top, "Homogeneous, Asymptotically Reliable Serial Production Lines: Theory and a Case Study," IEEE Transactions on Automatic Control, vol. 35, pp. 524-534, 1990.

[75] J. Li, "Throughput Analysis in Automotive Paint Shops: A Case Study," IEEE Transaction on Automation Science and Engineering, vol. 1, pp. 90-98, 2004.

[76] J. Li, D.E. Blumenfeld and S.P. Marin, "Manufacturing System Design to Improve Quality Buy Rate: An Automotive Paint Shop Application Study," IEEE Transactions on Automation Science and Engineering, vol. 4, pp. 75-79, 2007.

[77] J. Arinez, S. Biller, S.M. Meerkov and L. Zhang, "Quality/Quantity Improvement in an Automotive Paint Shop: A Case Study," to appear in IEEE Transactions on Automation Science and Engineering, 2009.

[78] F.K. Pil and S. Rothenberg, "Environmental Performance as a Driver of Superior Quality," Production and Operations Management, vol. 12, pp. 404-415, 2003. 
[79] A. King and M.M. Lenox, "Lean or Green? An Empirical Examination of the Relationship between Lean Production and Environmental Performance," Production and Operations Management, vol. 10, pp. 244-256, 2001. 


\section{VITA}

Name: Junwen Wang

Date of Birth: September 28, 1982

Place of Birth: Ezhou, China

\section{Education:}

M.S. in Electrical Engineering, conferred by Graduate University of Chinese Academy of Sciences in 2007,

B.S. in Electrical Engineering, conferred by University of Science and Technology of China in 2004.

\section{Scholastic Honors:}

- Finalist of Best Paper Award, IEEE Conference on Automation Science and Engineering, Bangalore, India, 2009.

- Third Prize in National Graduate Mathematical Contest in Modeling, China, 2006.

- First Prize in Mathematical Contest in Modeling of Northeast China, 2006.

- Outstanding Student Scholarships in University of Science and Technology of China, 2000, 2001, 2002, 2003. 


\section{Publications:}

\section{Book chapter}

- J. Wang and J. Li, "Bottleneck Sequence with respect to Quality in Flexible Manufacturing Systems: A Systems Approach," Chapter 11 in Advances in Systems Theory: Control, Communication Network, Production Systems and Rational Behavior, P.T. Kabamba and J. Li Ed., pp. 263-282, WingSpan Press, Livermore, CA, 2009.

\section{Journal articles (published/accepted)}

- J. Wang, Y. Hu and J. Li, "Transient Analysis to Design Buffer Capacity in Dairy Filling and Packing Production Lines," Journal of Food Engineering, vol. 98, pp. 1-12, 2010.

- J. Wang, J. Li, J. Arinez and S. Biller, "Product Sequencing with respect to Quality in Flexible Manufacturing Systems with Batch Operations," to appear in IEEE Transactions on Automation Science and Engineering, DOI: 10.1109 /TASE.2009.2039133, 2010.

- J. Wang, J. Li, J. Arinez, S. Biller and N. Huang, "Quality Analysis in Flexible Manufacturing Systems with Batch Productions: Performance Evaluation and Non-Monotonic Properties," to appear in IEEE Transactions on Automation Science and Engineering, DOI: 10.1109/TASE.2009.2029077, 2010.

- J. Wang, J. Li and N. Huang, "Optimal Vehicle Batching and Sequencing to Reduce Energy Consumption and Atmospheric Emissions in Automotive Paint 
Shops," to appear in International Journal of Sustainable Manufacturing, 2010.

- S. Brenner, Z. Zeng, Y. Liu, J. Wang, J. Li and P.K. Howard, "Modeling and Analysis of Emergency Department at University of Kentucky Chandler Hospital Using Simulations," to appear in Journal of Emergency Nursing, DOI: 10.1016/J.JEN.2009.07.018, 2010.

\section{Journal articles (under review)}

- J. Wang, J. Li, J. Arinez and S. Biller, "Quality Improvability with respect to Product Sequencing in Flexible Manufacturing Systems: Theory and A Case Study," submitted to IEEE Transactions on Automation Science and Engineering.

- J. Wang, J. Li and P.K. Howard, "An Analytical Framework for Modeling and Analysis of Hospital Emergency Department," submitted to IIE Transactions on Healthcare Systems Engineering.

- C.P. Arenas Guerrero, J. Wang, J. Li, J. Arenas, S. Biller, N. Huang and G. Xiao, "Production System Design to Reduce Energy Consumption: A Case Study in Automotive Paint Shop," submitted to International Journal of Production Research. 